University of South Florida

DIGITAL COMMONS

Digital Commons @ University of

@ UNIVERSITY OF SOUTH FLORIDA

South Florida

$9-2001$

\title{
Development of A Large-Scale Laboratory Facility for Sediment Transport Research
}

David G. Hamilton

U.S. Army Engineer Research and Development Center

Bruce A. Ebersole

U.S. Army Engineer Research and Development Center

Ernest R. Smith

U.S. Army Engineer Research and Development Center

Ping Wang

University of South Florida, pwang@usf.edu

Follow this and additional works at: https://digitalcommons.usf.edu/gly_facpub

Part of the Geology Commons

\section{Scholar Commons Citation}

Hamilton, David G.; Ebersole, Bruce A.; Smith, Ernest R.; and Wang, Ping, "Development of A Large-Scale Laboratory Facility for Sediment Transport Research" (2001). Geology Faculty Publications. 251.

https://digitalcommons.usf.edu/gly_facpub/251

This Statistical Report is brought to you for free and open access by the Geology at Digital Commons @ University of South Florida. It has been accepted for inclusion in Geology Faculty Publications by an authorized administrator of Digital Commons @ University of South Florida. For more information, please contact digitalcommons@usf.edu. 


\section{Development of a Large-Scale Laboratory} Facility for Sediment Transport Research

David G. Hamilton, Bruce A. Ebersole, Ernest R. Smith, and Ping Wang

US Army Corps of Engineers ${ }_{\circledast}$ Engineer Research and Development Center

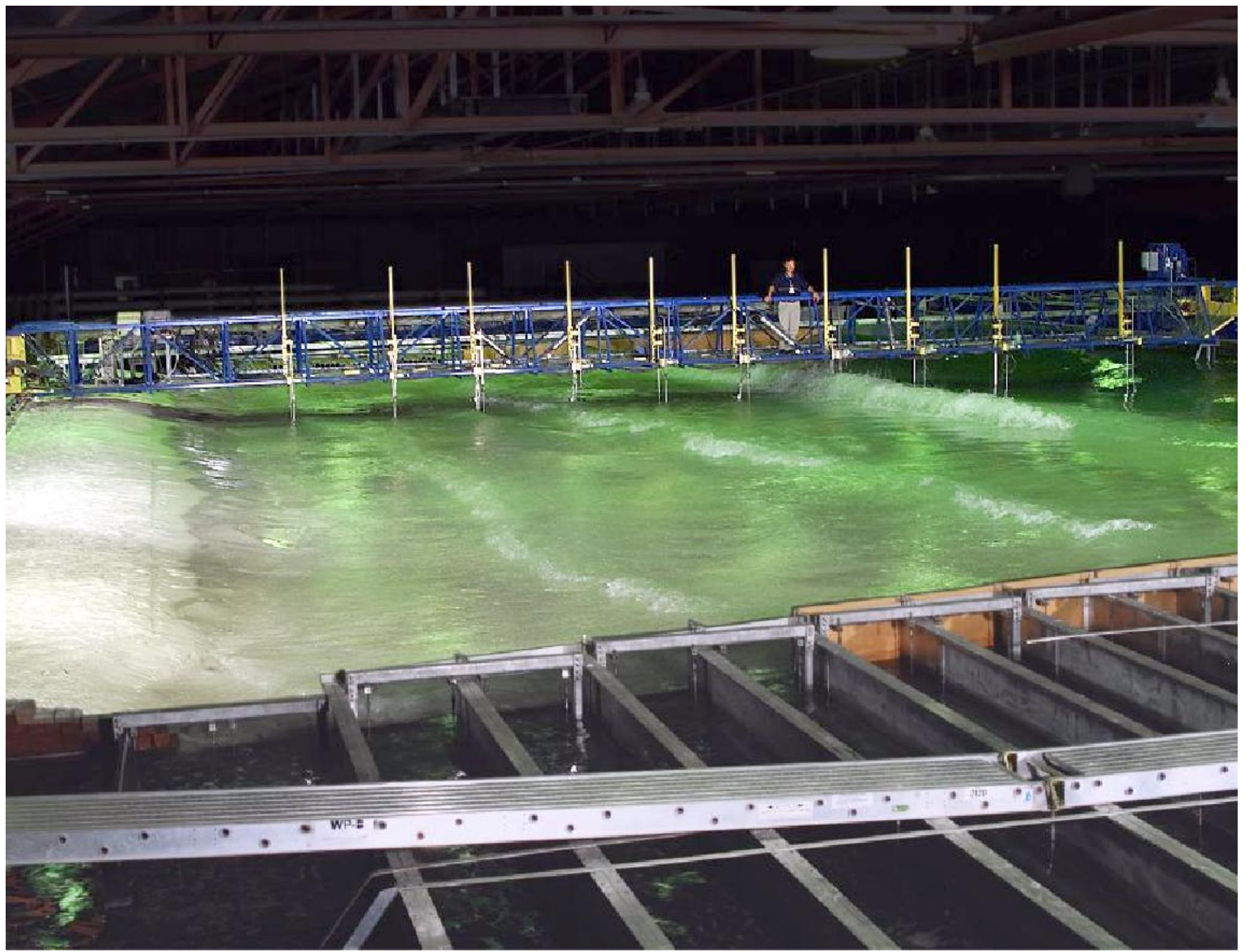


The contents of this report are not to be used for advertising, publication, or promotional purposes. Citation of trade names does not constitute an official endorsement or approval of the use of such commercial products.

The findings of this report are not to be construed as an official Department of the Army position, unless so designated by other authorized documents. 


\section{Development of a Large-Scale Laboratory Facility for Sediment Transport Research}

by David G. Hamilton, Bruce A. Ebersole, Ernest R. Smith, Ping Wang

Coastal and Hydraulics Laboratory

U.S. Army Engineer Research and Development Center 3909 Halls Ferry Road

Vicksburg, MS 39180-6199

Final report

Approved for public release; distribution is unlimited

Prepared for U.S. Army Corps of Engineers

Washington, DC 20314-1000 


\section{Contents}

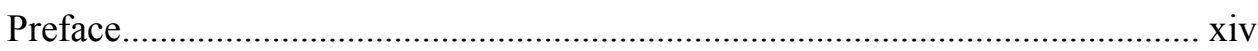

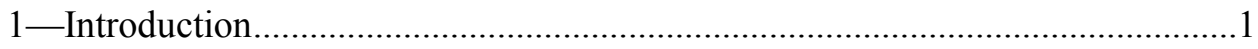

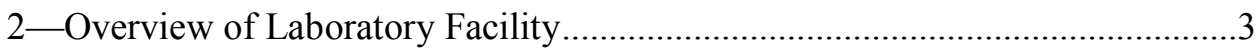

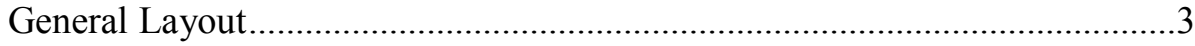

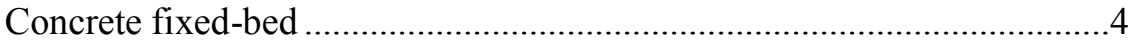

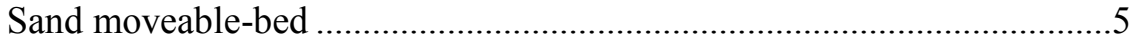

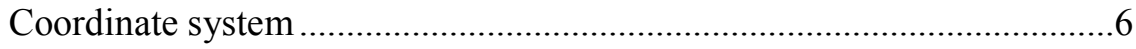

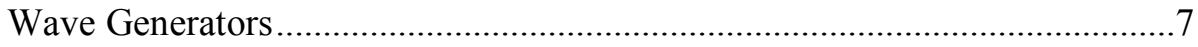

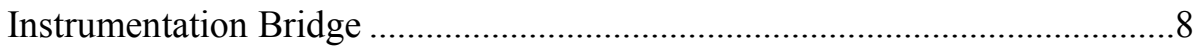

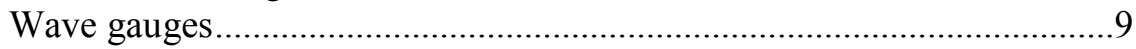

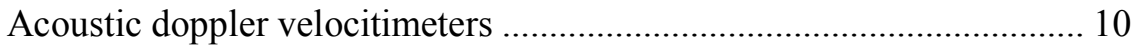

Fiber-optic backscatter sensors ........................................................... 10

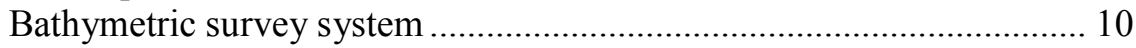

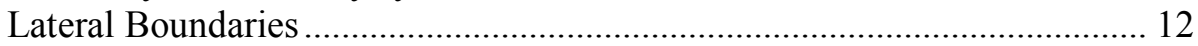

Control and Data Acquisition Room ......................................................... 12

3-Longshore Current Recirculation System ................................................... 14

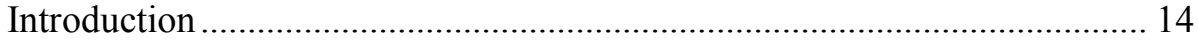

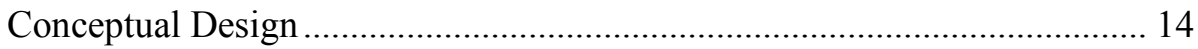

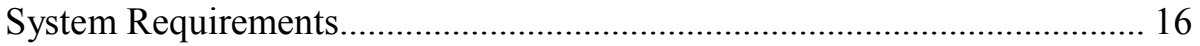

Numerical Simulation of Longshore Current.............................................. 17

Description of the numerical model...................................................... 17

Validation using dye measurements ................................................... 18

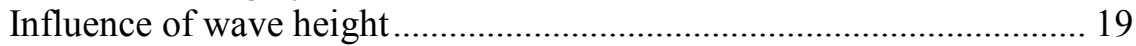

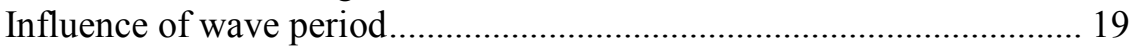

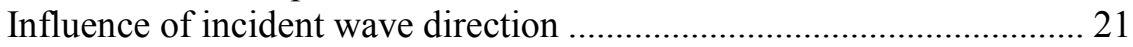

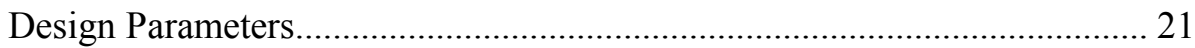

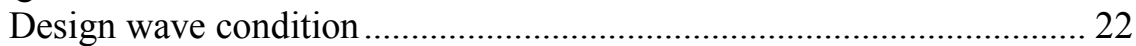

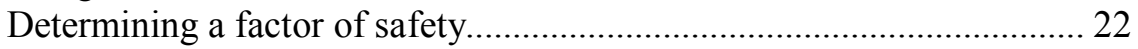

Allowance for maximum depth of erosion ........................................... 22

Allowance for variable water level ............................................................. 23

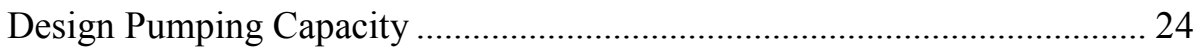

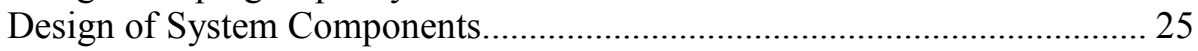

Vertical turbine pumps and motors.................................................... 26

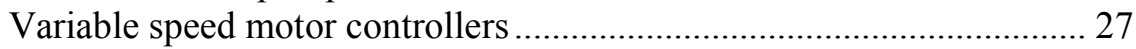

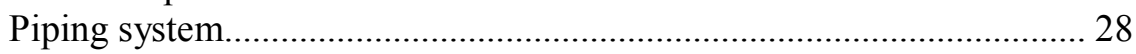

Flow measurement system ................................................................ 29 


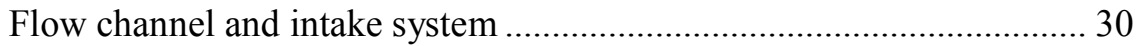

Hydraulic Characteristic Curves.............................................................. 30

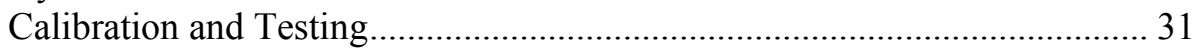

Relationship between pump discharge and frequency .......................... 31

Pumping steadiness and measurement repeatability …............................ 33

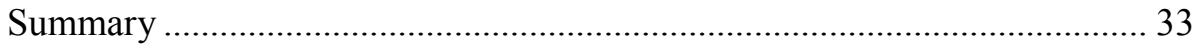

4 - Sediment Trapping and Handling Systems ................................................... 35

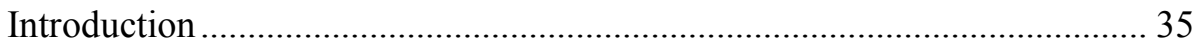

Design of Sediment Trapping System ....................................................... 36

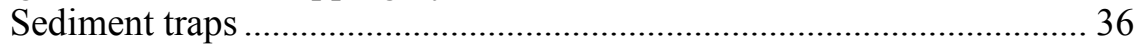

Trap support frames and degrees of freedom ........................................ 37

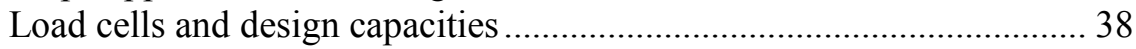

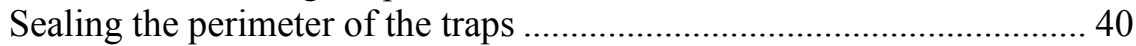

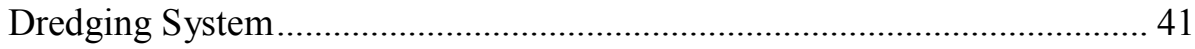

Static Performance Tests .............................................................................. 41

Tests with traps empty and the facility drained ..................................... 41

Removing air bubbles from beneath the rubber seals ............................ 43

Tests with the facility full of water .......................................................... 44

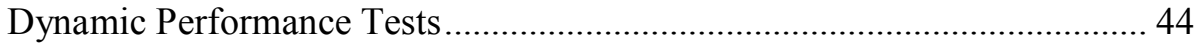

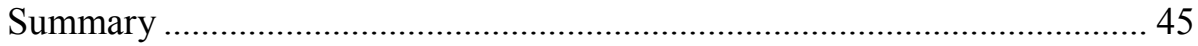

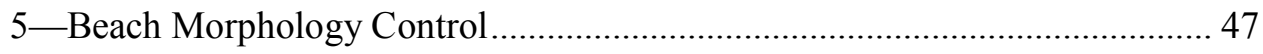

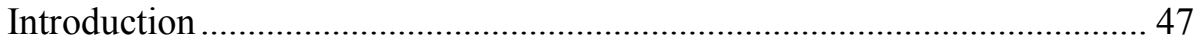

Maintaining Straight and Parallel Contours ….............................................. 47

Adjustable Boundary Elevation................................................................. 51

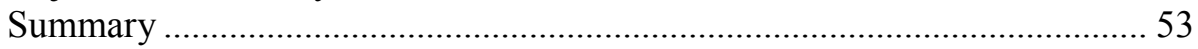

6-Experiment Control, Data Acquisition and Analysis Package........................ 54

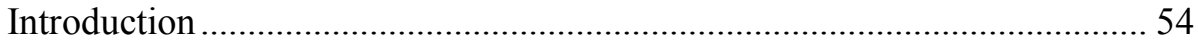

MTS Control and Data Acquisition System.................................................5 54

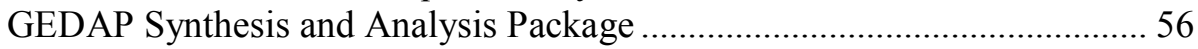

Display System for ADV Digital Data........................................................ 59

Control System for Vertical Turbine Pumps.............................................. 61

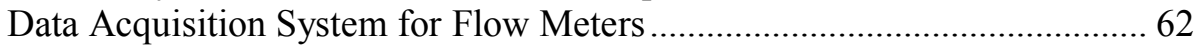

Data Acquisition System for Sediment Traps ................................................ 62

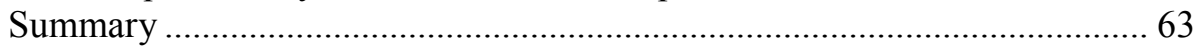

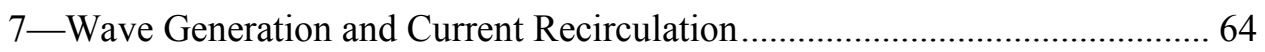

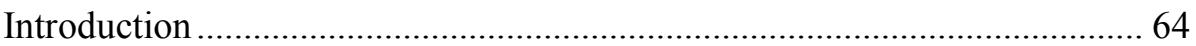

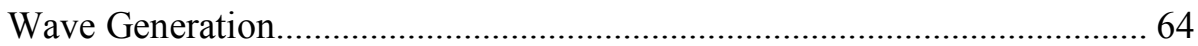

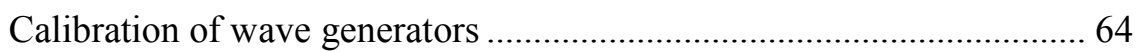

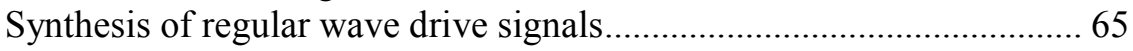

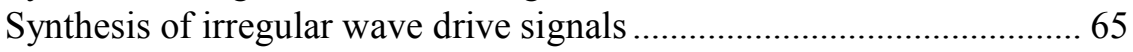

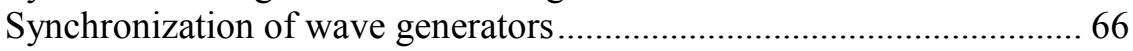

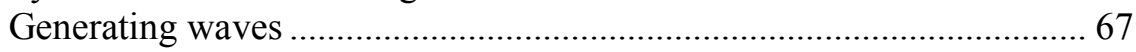

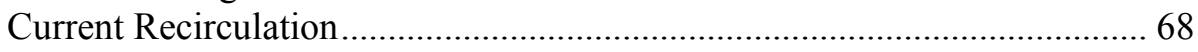

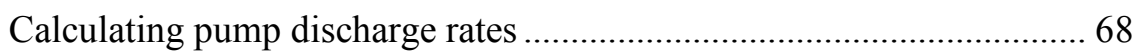

Setting the speed of the pumps ........................................................... 70

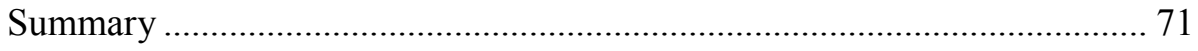


8-Sensor Calibration, Measurement and Data Analysis ................................. 72

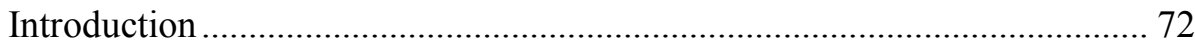

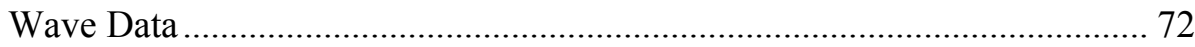

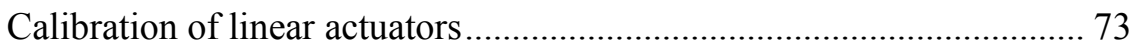

Calibration of wave gauges .................................................................. 74

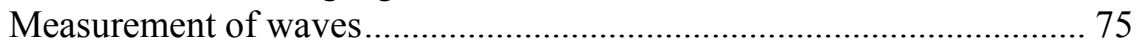

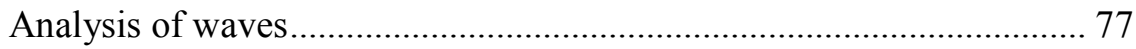

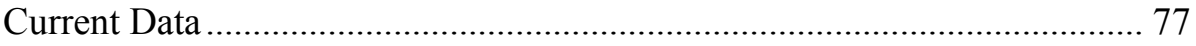

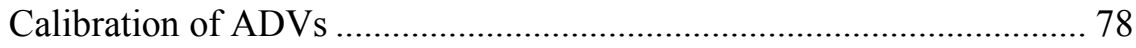

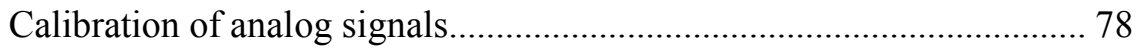

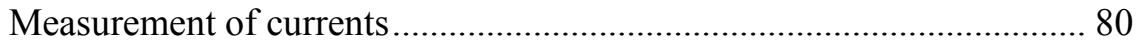

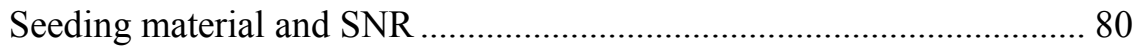

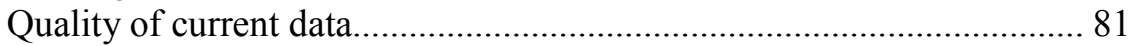

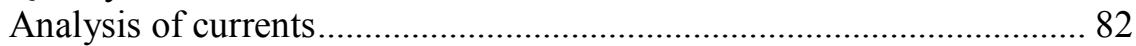

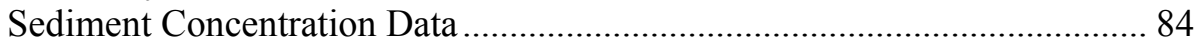

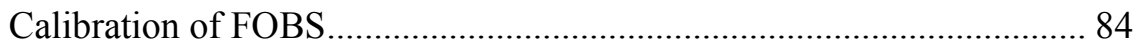

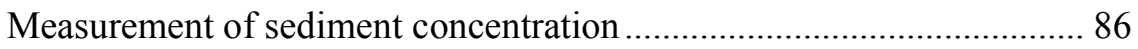

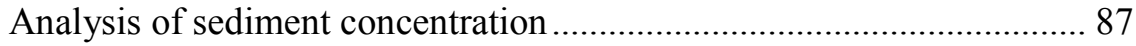

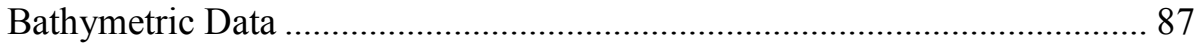

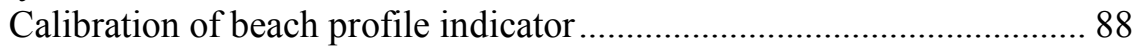

Measurement of beach profiles ......................................................... 90

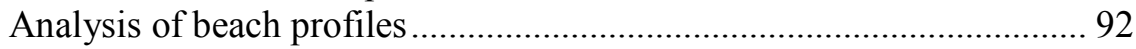

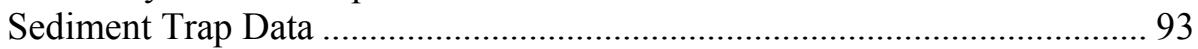

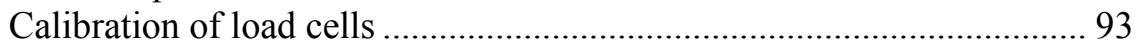

Measurement of sediment trap data ...................................................... 94

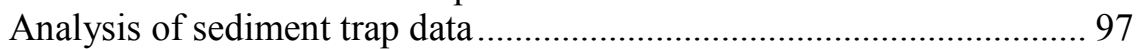

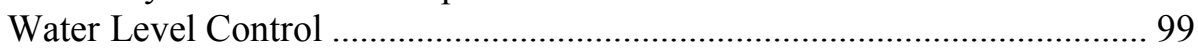

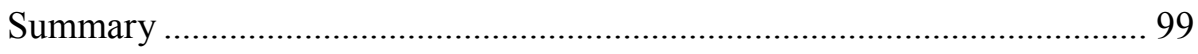

9-Establishing Uniform Longshore Currents ............................................... 101

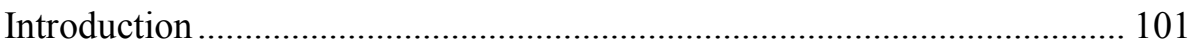

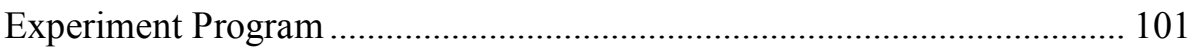

Measurement Methodology .................................................................... 103

Procedure for Tuning the Longshore Current ................................................. 104

Iterative examination of the longshore current distribution.................. 105

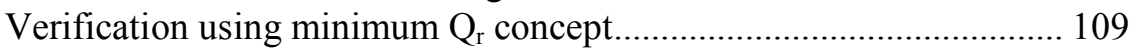

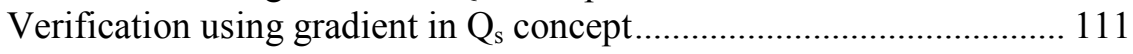

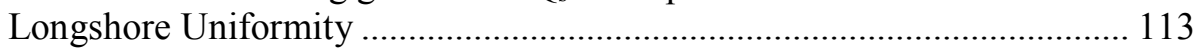

Longshore Current Steadiness and Repeatability ...................................... 119

Application to Sediment Transport Experiments ...................................... 120

10 - Longshore Sediment Transport Experiments ............................................ 122

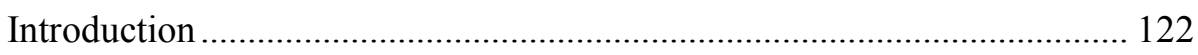

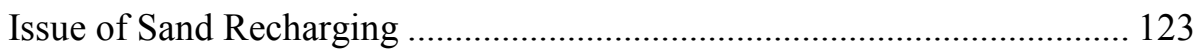

Procedures for Executing Experiments ................................................. 124

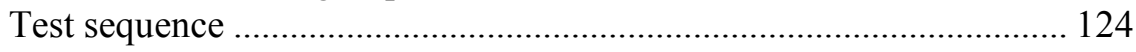

Instrumentation and measurement scheme.......................................... 125

Complexities of pumping the proper longshore current....................... 126

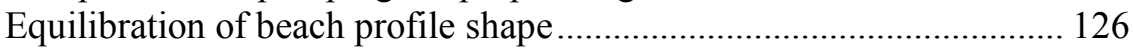

Steadiness and repeatability of measurements ................................... 127 


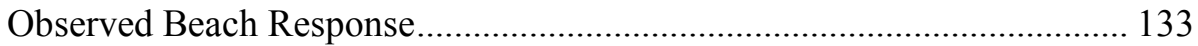

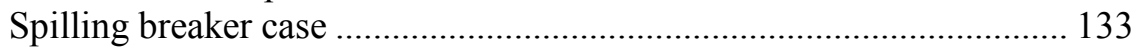

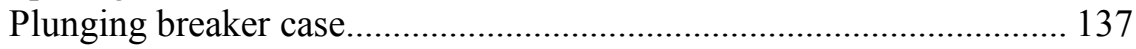

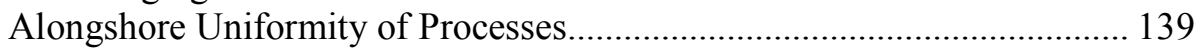

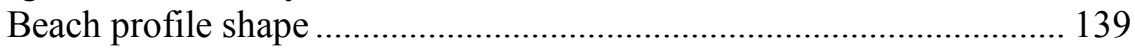

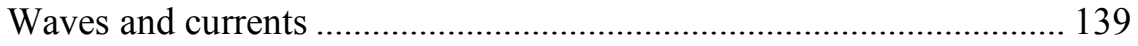

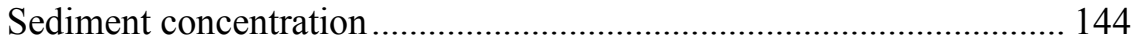

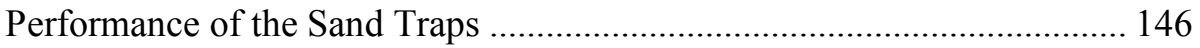

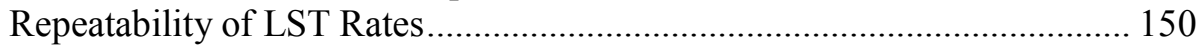

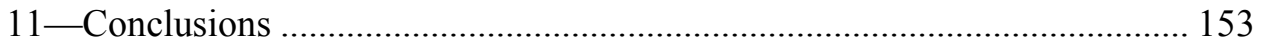

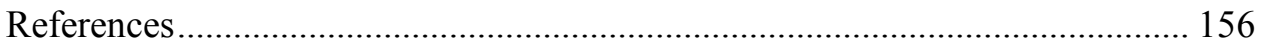

Appendix A-Hydrodynamic Data from Fixed-bed Experiments.......................A1

Appendix B —Photographs of the LSTF.........................................................

SF 298

\section{List of Figures}

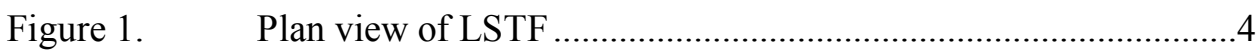

Figure 2. View of the LSTF during a sediment transport

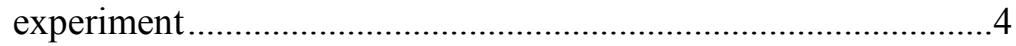

Figure 3. Grain size distribution of sand used in initial

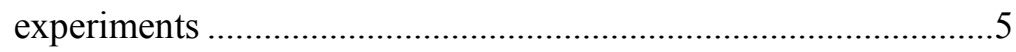

Figure 4. Profile view of LSTF showing the concrete and sand

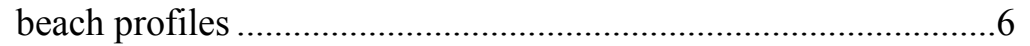

Figure 5. Synchronized wave generators creating long-crested

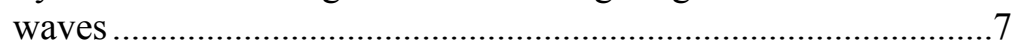

Figure 6. Performance of wave generators for a water depth of

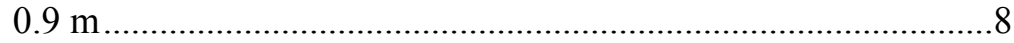

Figure 7. View of co-located sensors on instrumentation bridge .................9

Figure 8. Beach profile indicator resting on sand beach with facility drained

Figure 9. View of the LSTF from inside the data acquisition and

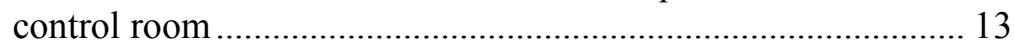

Figure 10. Seven types of longshore current recirculation systems ............ 15

Figure 11. Influence of wave height on required longshore current

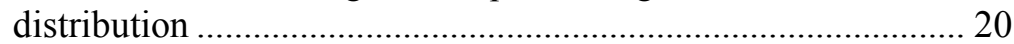


Figure 12. Influence of wave period on required longshore current distribution

Figure 13. Influence of wave direction on required longshore current distribution.

Figure 14. Influence of water depth on required longshore current distribution

Figure 15. Required and design pumping capacity of the longshore current recirculation system.

Figure 16. Oblique view of twenty vertical turbine pumps and motors

Figure 17. Variable speed motor controller with digital human interface

Figure 18. System No. 10: Pump performance and characteristic pipe curves

Figure 19. System No. 10: Relationship between pump discharge and frequency of the motor controller.....

Figure 20. Twenty sediment traps are located inside of downdrift flow channels

Figure 21. Two sediment traps are suspended from a set of support frames via stainless steel cables and load cells 38

Figure 22. Stainless steel, splash-proof load cells are bolted to the bottom of the support frames to support the sediment traps. A neoprene rubber seal is used between the flow channel walls and the sediment traps

Figure 23. Design capacity of the sediment traps in terms of the total load cell capacity and the maximum submerged weight of sand that each trap can hold based on the volumetric capacity of each trap.

Figure 24. Statistical parameters normalized by the average value in the time series, obtained from each load cell during tareweight tests conducted with the traps empty and the facility drained

Figure 25. Standard deviation of load cell readings measured during tare-weight tests conducted with the traps empty and the facility drained

Figure 26. Standard deviation of load cell readings measured during dynamic performance tests using both regular and irregular waves 46

Figure 27. Sand is removed from a temporary storage area, located in the updrift, offshore corner of the facility. This 
compartment will hold 5 to $10 \mathrm{~m}^{3}$ of sand. If necessary, sand can be pumped from the sediment traps directly into this storage area during the dredging process. However, during a typical experiment, the sand in the traps is pumped directly onto the updrift end of the beach

Figure 28. Prior to each experiment, the facility is drained, and a four-wheel drive tractor, with a front-end loader and a rear box-blade, is used to grade the sand beach to the proper beach profile

Figure 29. Stainless steel rods are permanently installed every $1.0 \mathrm{~m}$ along the updrift side of the bridge. The rods are lowered to preselected elevations to define the shape of the proper beach profile

Figure 30. The vertical rods are used as indicators to help the tractor operator maintain straight and parallel beach contours. In this figure, the bridge is moving to the left, and the bottom end of the vertical rod furthest offshore is deflected to the right as it drags along the beach. The depth of the groove remaining in the sand indicates how much the beach should be lowered in that region

Figure 31. Fine-tuning of the initial beach profile is achieved using a hand held shovel and rake, especially in regions where it is difficult to use the tractor, such as the steep front slope of the foreshore berm 50

Figure 32. Bathymetric plot following rebuilding of the beach ................. 51

Figure 33. Bathymetric plot following 230-min of plunging waves ........... 51

Figure 34. The elevation of the lateral boundaries is adjusted using bricks. Initially, the bricks are set as close as possible to match the initial beach profile. During the experiment, the downdrift boundary is observed carefully, and if necessary, bricks are removed or installed so that the downdrift brick boundary has almost the same profile shape as the average profile along the beach

Figure 35. Main panel of the MTS control software ….............................. 55

Figure 36. Block diagram of the GEDAP system (Miles 1997) ................ 58

Figure 37. GEDAP on-line documentation listing the program classes and subroutine libraries (Miles 1997).... 60

Figure 38. Real-time display screen for ADV digital data (SonTek 1997)

Figure 39. Wave programs control panel in the MTS control software 
Figure 40. Measurement of wave, current, and sediment concentration data

Figure 41. Summary panel of wave gauge calibrations in MTS control software 75

Figure 42. Data recorder panel in MTS control software. 76

Figure 43. Relationship between the output voltage range from the $\mathrm{ADV}$ s and the range of the $\mathrm{A} / \mathrm{D}$ converters with respect to engineering units

Figure 44. Existing sensors panel in the MTS control software is used to scale the analog output signals from voltage into engineering units

Figure 45. A/D-D/A converters panel in the MTS control software is used to adjust the gain and offset values for the ADV analog output signals

Figure 46. Comparison of longshore current measured with ADV No. 2, before and after removing spikes caused by air bubbles in shallow water.....

Figure 47. Calibration results for FOBS No. 1 on Vertical Array No. 1 85

Figure 48. Calculation of a vertical sediment concentration profile 88

Figure 49. Calibration of beach profiler indicator is verified on benchmark

Figure 50. Calibration panel in the MTS control software for the beach profile indicator

Figure 51. Operation panel in the MTS control software for the fully automated bathymetric survey system

Figure 52. Average sediment trap weights for Test1H_Case2, showing the amount of sand that had accumulated on the neoprene rubber seals.

Figure 53. Cross-shore distribution of longshore sediment transport measured during the $3.33 \mathrm{hr}$ duration of test segment Test1H_Case2

Figure 54. Instantaneous submerged weight of Sediment Trap No. 2 during the first $2 \mathrm{hr}$ of test segment Test1H_Case2

Figure 55. Oblique view of the Large-scale Sediment Transport Facility. 102

Figure 56. Plan view of the LSTF and a conceptual diagram of longshore flow conditions. 103 
Figure 57. Progression of pump settings for regular wave experiments

Figure 58. Test 6D - under-pumping: Measured longshore current distribution at $\mathrm{Y}=19,27$, and $35 \mathrm{~m}$.

Figure 59. Test $6 \mathrm{~J}$ - over-pumping: Measured longshore current distribution at $\mathrm{Y}=19,27$, and $35 \mathrm{~m}$

Figure 60. Test $6 \mathrm{~N}$ - proper pumping: Measured longshore current distribution at $\mathrm{Y}=19,27$, and $35 \mathrm{~m}$.

Figure 61. Surf zone and internal recirculation flow rates at Y27 for 15 regular wave experiments

Figure 62. Surf zone and internal recirculation flow rates at Y27 for five irregular wave experiments

Figure 63. Regular wave test series: Longshore variation in surf zone flow rates, Qs

Figure 64. Irregular wave test series: Longshore variation in surf zone flow rates, Qs

Figure 65. Regular wave Test $6 \mathrm{~N}$ : Longshore uniformity of hydrodynamics vs length of surf zone.

Figure 66. Longshore uniformity of surf zone

Figure 67. Irregular wave Test 8E: Longshore uniformity of hydrodynamics vs length of surf zone

Figure 68. High degree of longshore hydrodynamic uniformity for irregular wave case.

Figure 69. Irregular wave Test 8E: Sequential measurements of mean longshore current at $\mathrm{Y}=27 \mathrm{~m}$

Figure 70. Irregular wave Test 8E: Distribution of mean longshore current at downstream boundary

Figure 71. Evolution of beach profile shape for the plungingbreaker case

Figure 72. Repetitive measurements of mean longshore current during a single-wave run

Figure 73. Measurements of significant wave height for successive wave runs at the same cross-shore transect for the spilling-breaker case

Figure 74. Measurements of significant wave height for successive wave runs at the same cross-shore transect for the plunging-breaker case 
Figure 75. Measurements of mean longshore current for successive wave runs at the same cross-shore transect for the spilling-breaker case

Figure 76. Measurements of mean longshore current for successive wave runs at the same cross-shore transect for the plunging-breaker case.

Figure 77. Measurements of sediment concentration for successive wave runs at the same cross-shore transect, for the spilling-breaker case (top panel shows measurements from incipient breaker zone; bottom panel shows measurements from midsurf zone)

Figure 78. Measurements of sediment concentration for successive wave runs at the same cross-shore transect for the plunging-breaker case (top panel shows measurements from incipient breaker zone; bottom panel shows measurements from midsurf zone)

Figure 79. Beach morphology after $6 \mathrm{hr}$ of wave activity for the spilling-breaker case (elevations are in meters)

Figure 80. Beach morphology after nearly $10 \mathrm{hr}$ of wave activity for the spilling-breaker case (elevations are in meters)

Figure 81. Change in morphology after nearly $10 \mathrm{hr}$ of wave activity for the spilling-breaker case (elevation changes are in meters).

Figure 82. Beach morphology after nearly $10 \mathrm{hr}$ of wave activity for the plunging-breaker case (elevations are in meters)

Figure 83. Alongshore variation in beach profile shape for the spilling-breaker case (in the upper panel, still-water level is at an elevation of $0.9 \mathrm{~m}$ ).

Figure 84. Alongshore variation in beach profile shape for the plunging-breaker case (in upper panel, still-water level is at an elevation of $0.9 \mathrm{~m}$ )

Figure 85. Alongshore variation in significant wave height for the spilling-breaker case

Figure 86. Alongshore variation in significant wave height for the plunging-breaker case.

Figure 87. Alongshore variation in mean longshore current for the spilling-breaker case 143

Figure 88. Alongshore variation in mean longshore current for the plunging-breaker case.

Figure 89. Alongshore variation in time-averaged mean sediment concentration for the spilling-breaker case (top panel 
shows results from the incipient breaker zone; bottom panel shows results from the midsurf zone)

Figure 90. Alongshore variation in time-averaged mean sediment concentration for the plunging-breaker case (top panel shows results from the incipient breaker zone; bottom panel shows results from the midsurf zone)

Figure 91. Weight of sand that accumulated on the rubber seals around the traps

Figure 92. Volume change computed for each profile 148

Figure 93. Total LST rates computed with and without corrections to account for anomalous volume change adjacent to the downdrift boundary for the spilling-breaker case

Figure 94. Total LST rates computed with and without corrections to account for anomalous volume change adjacent to the downdrift boundary for the plunging-breaker case

Figure 95. Cross-shore distribution of the LST rate for the spillingbreaker case

Figure 96. Cross-shore distribution of the LST rate for the plunging-breaker case.

Figure A-1. Test 6N: Vertical structure of the mean longshore current at Y27

Figure A-2. Test 8E: Vertical structure of the mean longshore current at $\mathrm{Y} 27$

Figure B-1. The concrete beach has a 1:30 slope

Figure B-2. Four piston-type wave generators are shown with the facility drained B2

Figure B-3. The instrumentation bridge is $21 \mathrm{~m}$ long and spans the entire width of the beach

Figure B-4. Wave gauges and current meters are co-located at various distributions across the width of the surf zone. B3

Figure B-5. The offshore wave gauges are mounted to stainless steel tripods along the toe of the sand beach

Figure B-6. The 20 vertical turbine pumps are mounted inside of the 20 independent sumps to ensure vortex free operation at maximum discharge

Figure B-7. Each pump in the Longshore Current recirculation system pumps water through one of two parallel pipelines 
Figure B-8. Pump No. 10 forces through either a 50- or a 100-mmdiam in-line flow sensor

Figure B-9. View of the wave guide at the downstream end of the beach. B5

Figure B-10. View of the wave guide at upstream end of the beach B6

Figure B-11. A matrix of PVC pipe was installed immediately upstream of each vertical turbine pump to absorb residual wave energy and to minimize wave reflection in the flow channels

Figure B-12. Two 400-mm-diam polyvinyl chloride pipes were installed in the concrete beach to passively recirculate the longshore current that flows in the swash zone.

Figure B-13. An overall view of the facility after the sand beach has been graded to have straight and parallel depth contours

\section{List of Tables}

Table 1. Vertical Turbine Pump and Motor Specifications .................... 27

Table 2. Dimensions and Capacities of the 20 Sediment Traps............... 37

Table 3. Calculation of Discharge Rates for 20 Vertical Turbine

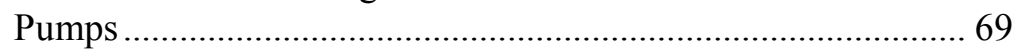

Table 4. Summary of Incident Wave Conditions ................................. 103

Table 5. Instrumentation and Sampling Scheme ….............................. 125

Table A-1. Primary Data Set from Regular Wave Test 6N.........................A1

Table A-2. Primary Data Set from Irregular Wave Test 8E........................A2

Table A-3. Summary of Wave and Water Level Conditions in the Surf Zone 


\section{Preface}

This report documents the development and initial applications of the Largescale Sediment Transport Facility (LSTF), which is located at the U.S. Army Engineer Research and Development Center (ERDC), Coastal and Hydraulics Laboratory (CHL), Vicksburg, MS. The LSTF was built to facilitate basic and applied research in the field of coastal sediment transport processes. Work reported here was funded by Work Unit 32870 "Large-Scale Laboratory Investigation of Longshore Sediment Transport," which is under the Coastal Sedimentation and Dredging Program, General Investigations Research and Development Program, U.S. Army Corps of Engineers (USACE). Mr. Barry W. Holliday, Headquarters USACE, was Technical Monitor for the program.

Considerable planning, design, and development of the LSTF was conducted by Mr. David G. Hamilton, Hydraulic Engineer, while under contract to ERDC, CHL, during the period October 1994 through May 2000. Mr. Hamilton was the lead engineer on the fixed-bed experiments that were performed in the LSTF. Mr. Bruce A. Ebersole, Chief, Coastal Processes Branch (CPB), ERDC, served as the Contracting Officer's Representative for the contract during this time, and he monitored planning, design, construction, and application of the facility. Ms. Julie D. Rosati, Research Hydraulic Engineer, CPB, was the Principal Investigator of the Large-Scale Laboratory Investigation of Longshore Sediment Transport work unit from FY94 through FY98. Mr. Ebersole was the Principal Investigator of this work unit from FY99 through FY00. Mr. Ernest R. Smith, Research Hydraulic Engineer, CPB, is presently the Principal Investigator for the work unit, and he is the lead engineer on the movable-bed investigations.

Dr. Ping Wang, Louisiana State University, is a research partner under contract to CHL and is assisting with the movable-bed experiments. This project is being conducted under the direction of Mr. Thomas W. Richardson, Acting Director, CHL.

Several other individuals contributed significantly to the development and maintenance of the LSTF. Mr. Homer C. Greer, Electronics Engineer, Operations Branch, Instrumentation Systems Development Division, Information Technology Laboratory (ITL), and Mr. David A. Daily, Senior Electronics Technician, ITL, are acknowledged for development and procurement of the instrumentation for the LSTF. Mr. Daily and Mr. Timothy E. Nisley, Electronics Technician, ITL, are acknowledged for their valuable assistance during execution of experiments and in maintenance of the instrumentation systems. In addition, Mr. Peter J. Neilans, Hydraulic Engineer, formerly CPB, is acknowledged for 
working closely with Mr. Hamilton in developing the longshore current recirculation system.

The authors also would like to acknowledge the contributions of Dr. Richard Whitehouse, who openly shared his experience in developing the Coastal Research Facility at HR Wallingford, United Kingdom. Dr. Paul Visser also provided valuable information concerning the experimental methodologies he used in experiments conducted at Delft University of Technology, the Netherlands. The authors also would like to acknowledge Mr. Carl Miller and Dr. Reggie Beach, Office of Naval Research, Field Research Facility, CHL, for the use of and assistance with their Fiber-optic Backscatter Sensors.

This report was prepared by Messrs. Hamilton, Ebersole, Smith, and Dr. Wang. The technical reviews of Ms. Rosati and Dr. Jane M. Smith, CPB, are greatly appreciated.

At the time of publication of this report, Dr. James R. Houston was Director of ERDC, and COL John W. Morris III, EN, was Commander and Executive Director.

The contents of this report are not to be used for advertising, publication, or promotional purposes. Citation of trade names does not constitute an official endoresement or approval of the use of such commercial products. 


\section{Introduction}

In performing its mission to maintain navigable waterways along U.S. coasts, the U.S. Army Corps of Engineers (USACE) regularly applies analytical and numerical models to estimate the total longshore sediment transport (LST) rate. Accurate prediction of LST rate is essential when predicting beach response in the vicinity of coastal structures, designing beach nourishment projects, and estimating sedimentation rates in navigation channels. For design applications with adequate field measurements, the commonly used CERC formula (Shore Protection Manual 1984) can be calibrated and applied to estimate total LST rates with reasonable confidence ( $+/-50$ percent error). However, for design applications without calibration data, the CERC formula provides only order-ofmagnitude accuracy. Very little information and guidance are available for defining the cross-shore distribution of LST.

To address deficiencies in existing methods for calculating LST, the Largescale Sediment Transport Facility (LSTF) has been constructed at the U.S. Army Engineer Research and Development Center's (ERDC) Coastal and Hydraulics Laboratory $(\mathrm{CHL})$. The intent for the facility is to reproduce certain surf zone processes found on a long, straight, natural beach in a finite-length wave basin. Ongoing work in the LSTF is part of a research program to improve USACE's capability to predict local and total LST rates and to evaluate errors associated with these predictions. The LSTF simulates nearshore hydrodynamic and sediment transport processes at a relatively large geometric scale, including situations where considerable sand is mobilized and transported in suspension.

Chapter 2 provides an overview of the LSTF, including a brief description of its major components. Chapters 3 and 4 provide much more detailed information about the design of both the longshore current recirculation and sediment trapping and handling systems. Chapter 5 discusses the procedures for molding beach morphology to the prescribed profile shape. Chapter 6 describes the automated system for controlling experiments and the data acquisition and analysis package that is used to process measured data. Chapter 7 describes operational procedures used for controlling the wave generators and pumps that force the longshore current distribution. Chapter 8 describes the calibration, measurement, and analysis of various types of data that are acquired during experiments: wave, current, sediment concentration, beach topography, and quantities of sand that accumulate in traps located at the downdrift boundary of the facility. Chapter 9 describes the critical iterative process for establishing the proper longshore current and summarizes results from regular and irregular wave longshore current experiments performed on a planar concrete beach. The 
success of this procedure is evaluated. Chapter 10 describes two initial movablebed experiments, which were designed to learn how to effectively conduct movable-bed experiments in the LSTF. The experiments also were designed and executed to address several research objectives, namely identifying the role of breaker type and beach morphology on LST processes. Differences in LST processes for spilling- and plunging-breakers were examined and results from the experiments are presented. Conclusions are made in Chapter 11, and the success of performing LST experiments in the LSTF is evaluated. Hydrodynamic data from fixed-bed experiments are presented in Appendix A, and photographs of the LSTF are given in Appendix B. 


\section{Overview of the Laboratory Facility}

\section{General Layout}

The Large-scale Sediment Transport Facility (LSTF) has dimensions of $30 \mathrm{~m}$ cross-shore by $50 \mathrm{~m}$ longshore by $1.4 \mathrm{~m}$ deep, as shown in Figure 1. A moveable-bed beach made of quartz sand, constructed on top of a concrete fixedbed beach, is located in the central region of the facility. Four synchronized wave generators are located offshore to generate unidirectional, long-crested waves. A rubble mound wave absorber is located behind the wave generators to minimize wave reflections. Longshore current and sediment are transported toward the 20 flow channels at the downstream end of the facility (left side of figure). Each of the 20 flow channels at the downdrift end contains a low profile, gravity-feed sediment trap to measure the rate of longshore sediment transport. The longshore current is externally recirculated from the downdrift end of the facility through 20 independent pump-and-piping systems, back to the upstream end of the facility (right side of figure). The flow is guided from the discharge locations to the updrift end of the beach by 20 flow channels at the upstream end of the facility. The longshore current recirculation system is essentially a closedloop system that continuously recirculates longshore current from the downstream to the upstream lateral boundary of the beach while waves are being generated.

This chapter provides a brief overview of the various components of the facility. The external longshore current recirculation system and the sediment trapping and handling system will be discussed in subsequent chapters because of the complexity and large number of components in these two systems. Figure 2 is a photograph of the LSTF taken during a recent moveable-bed sediment transport experiment. The offshore wave conditions during this experiment are $\mathrm{H}_{\mathrm{s}}=0.30 \mathrm{~m}$ and $\mathrm{T}_{\mathrm{p}}=3.0 \mathrm{sec}$ with a 10-deg angle of wave incidence. 


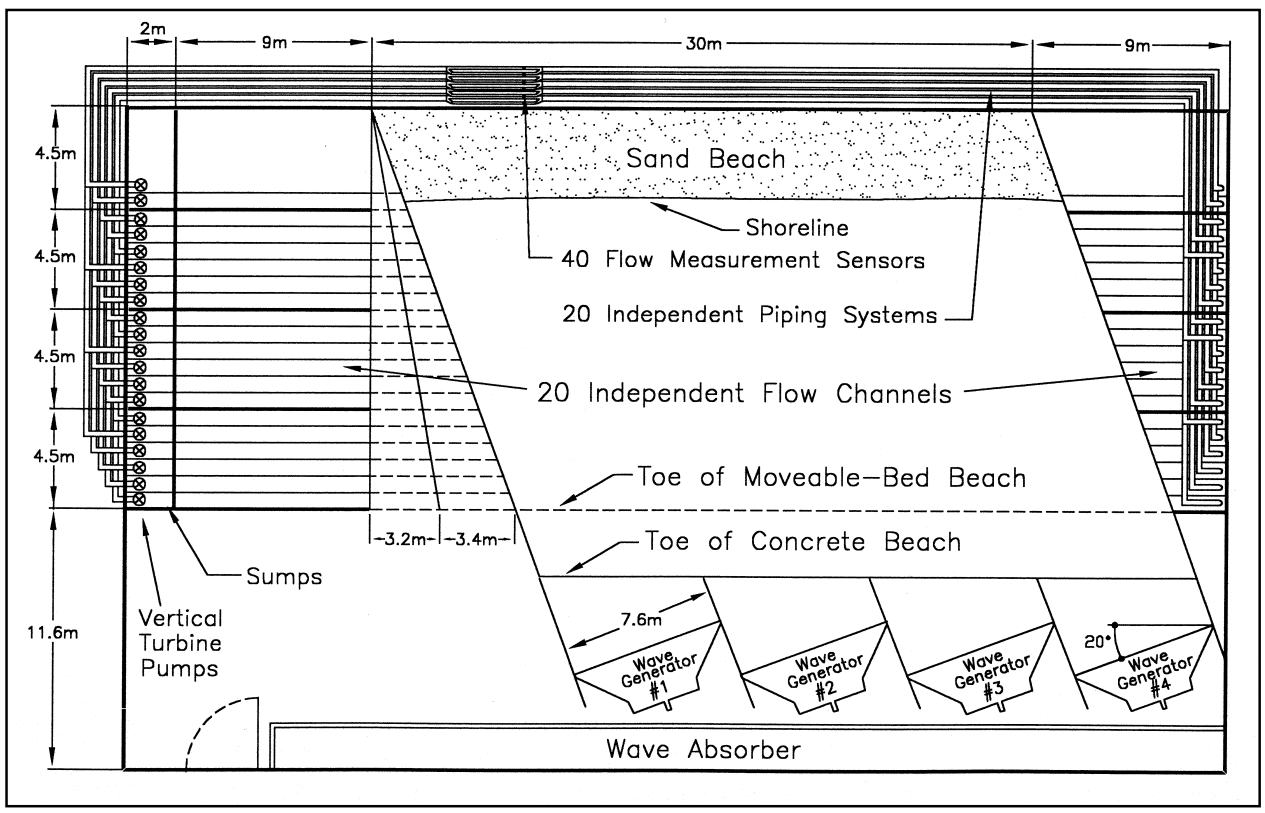

Figure 1. Plan view of the LSTF

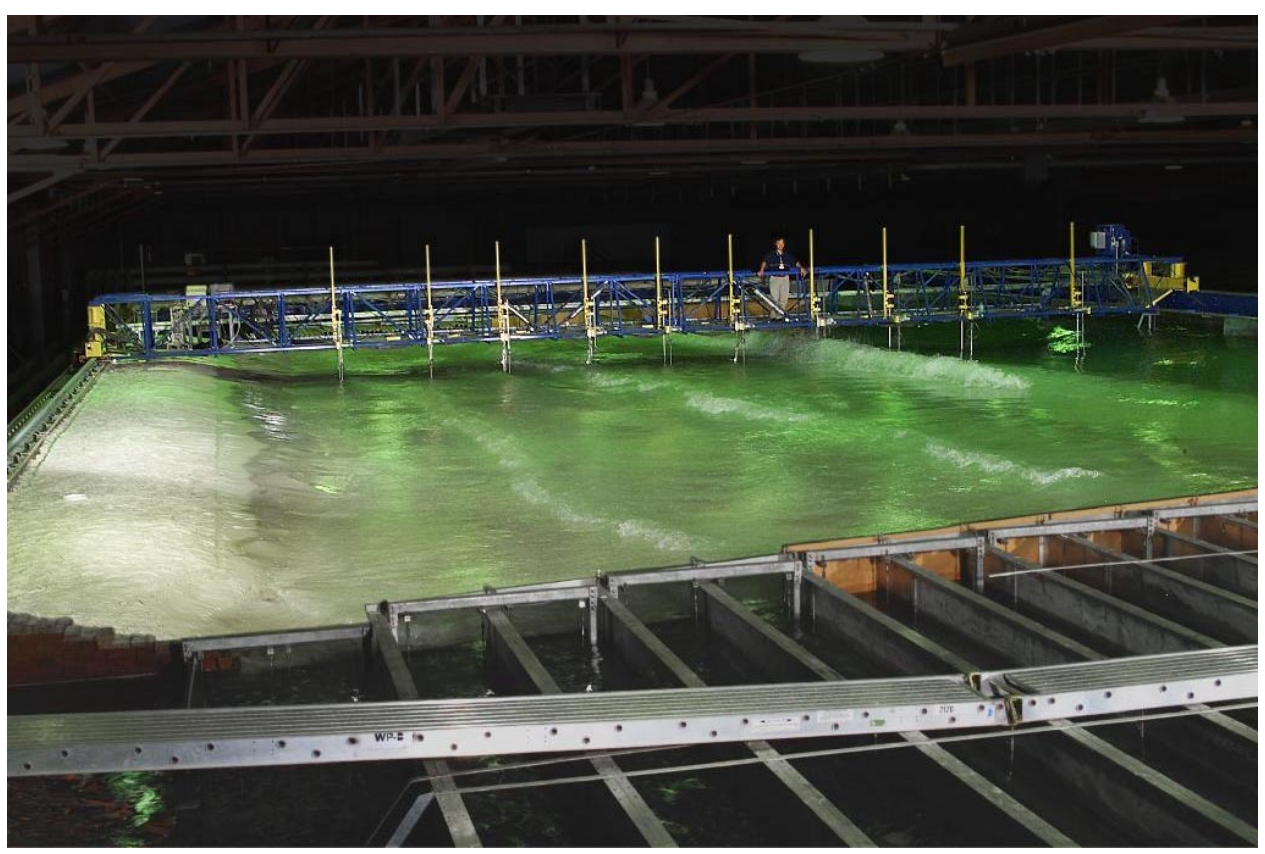

Figure 2. View of the LSTF during sediment transport experiment

\section{Concrete fixed bed}

The concrete beach has a longshore dimension of $31 \mathrm{~m}$ and a cross-shore dimension of $21 \mathrm{~m}$. The main section of the concrete beach has a constant slope of 1:30 and the toe of the beach slopes down to the basin floor at a 1:18 slope (Figure 3). Equations describing the shape of the fixed-bed beach are provided in 
Appendix A. The fixed-bed beach was constructed by accurately installing bottom elevation templates that are fixed to the floor of the wave basin. The space between the templates was filled with general-purpose sand and compacted, and then capped with a concrete veneer approximately $0.1 \mathrm{~m}$ thick. The surface of the concrete was broom finished to simulate the natural roughness of sand. Figure B-1, Appendix B, shows the concrete beach from the downstream end of the facility. Small longshore variations in beach elevation can have a significant influence on longshore current patterns (Putrevu, OltmanShay, and Svendsen 1995). Therefore, the beach was designed and carefully constructed with straight and parallel depth contours and a high degree of accuracy (estimated vertical variation of $\pm 2 \mathrm{~mm}$ ).

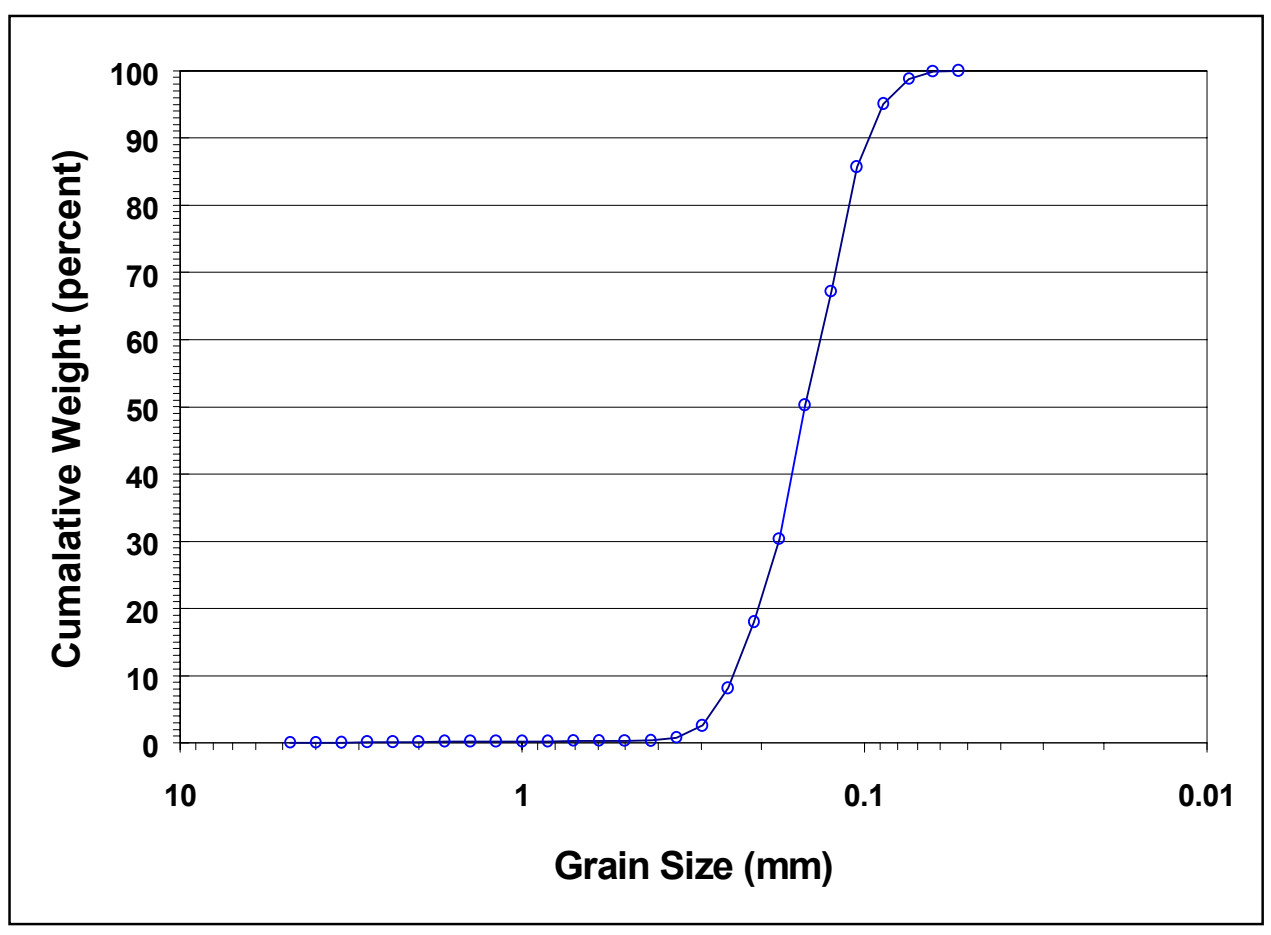

Figure 3. Grain size distribution of sand used in initial experiments

\section{Sand moveable bed}

One of the purposes of the LSTF is to quantify the effect of sediment size on longshore sediment transport. The facility was designed so that the moveablebed beach could be constructed with various sand sizes. The first phase of sediment transport experiments is being conducted on a moveable-bed beach consisting of approximately $125 \mathrm{~m}^{3}$ of very well-sorted quartz sand having a median grain size, $\mathrm{D}_{50}=0.15 \mathrm{~mm}$ (Figure 3 ). This is considered fine-grained sand according to the Wentworth classification system. An additional $25 \mathrm{~m}^{3}$ of sand is stored outside of the facility for future use.

At the beginning of the sediment transport experiments, the beach profile was graded to have the initial profile shape shown in Figure 4. This initial shape was determined based on results obtained from preliminary moveable-bed 
experiments and an analysis of the equilibrium beach profile for this grain size (Dean 1977). The height of the berm was selected so that the maximum excursion of wave runup in the swash zone would not reach the upper elevation of the berm. The front slope of the berm (approximately 1:5.5) was chosen based on the results of preliminary moveable-bed experiments. The main section of the beach was graded to have a constant slope of approximately 1:27, extending from the initial still-water shoreline, offshore to $16 \mathrm{~m}$ from the onshore boundary. The toe of the moveable-bed beach slopes down to the concrete beach on a 1:8 slope and has proven to be stable for the wave conditions tested. The objectives in designing this initial beach profile were to minimize the time required for the beach profile to approach equilibrium, and to simplify the shape of the profile so that the beach could be regraded to this initial shape on a regular basis. The calculated equilibrium beach profile for this sediment size also is shown in Figure 4 . The process of initially grading the beach to have straight and parallel contours and the procedure used to adjust the updrift and downdrift boundaries of the beach is discussed in Chapter 5 .

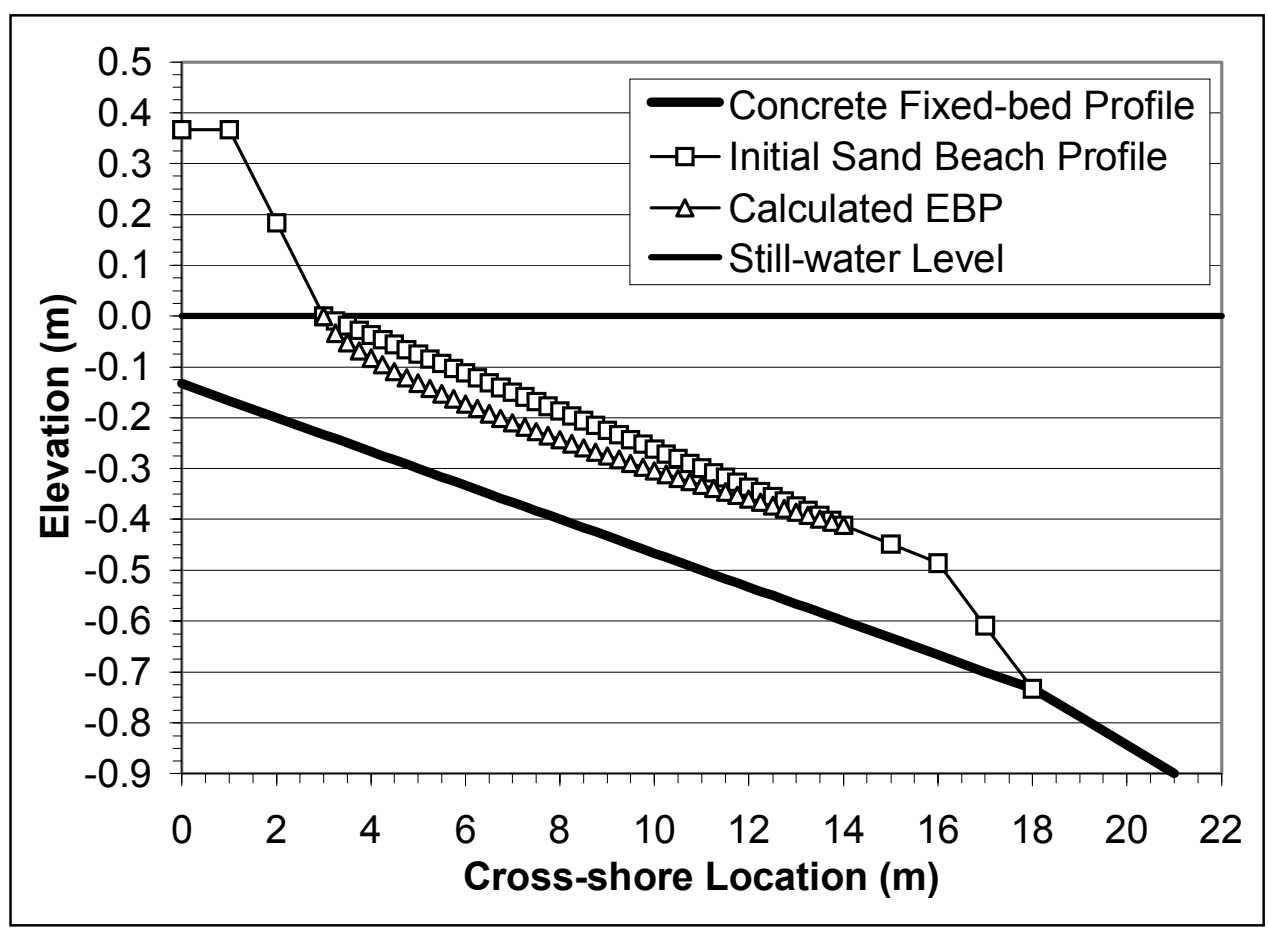

Figure 4. Profile view of LSTF showing the concrete and sand beach profiles

\section{Coordinate system}

The coordinate system used in the facility is right-handed, with the origin at the downstream, shoreward end of the wave basin. The positive $\mathrm{x}$-axis is directed offshore and is measured relative to the upper edge of the concrete beach slope. The positive y-axis is directed upstream. The z-axis is measured positively upward with the origin at the still-water level. The coordinate system was chosen based on future plans to extend the length of the facility. However, for simplicity, all downstream-directed longshore current speeds and flow rates 
presented in this report are given as positive numbers, even though the flow is in the negative $y$-direction.

\section{Wave Generators}

Regular and irregular waves are generated using four repositionable, pistontype wave generators. The waves are accurately generated using a digitally controlled servo electric drive system to control the position of the wave boards. Each wave generator has a 7.62-m-long wave board, so all four systems generate a total wave front of $30.5 \mathrm{~m}$ in length. The wave generators are synchronized to create unidirectional long-crested waves. Figure 5 is a photograph of the wave generators during a performance test to determine the maximum wave height that can be generated at various wave periods. The wave generators can be oriented at various angles ranging from 0 to 20 deg with respect to shore-normal. In Figure 4, the wave generators are positioned at $10 \mathrm{deg}$ to shore-normal. For oblique angles of wave attack, the wave generators are phase-shifted to maximize the crossshore dimension of the testing region. End baffles, located between each adjacent wave board, are used to guide the waves and prevent the formation of spurious waves caused from wave diffraction from the ends of each wave board and from wave energy reflected from the wave absorber located behind the wave generators.

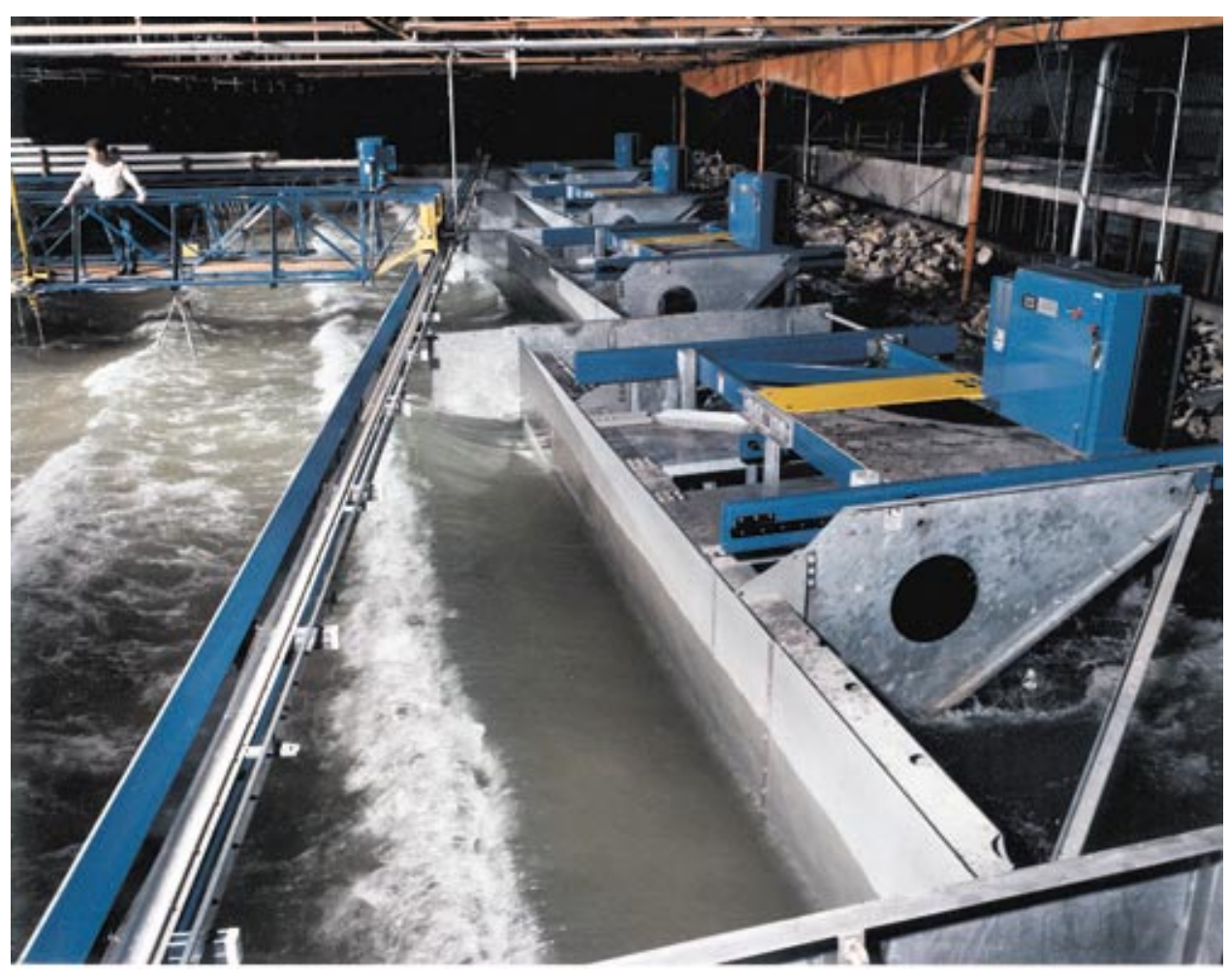

Figure 5. Synchronized wave generators creating long-crested waves

A performance curve for the wave generators is given in Figure 6 for a water depth of $0.9 \mathrm{~m}$ at the wave generators. There are six curves in this figure. The cresting-limit curve is based on linear wave theory for $\mathrm{H} / \mathrm{L}_{\mathrm{o}}=0.142$, where $\mathrm{H}$ is 
the wave height at the generator and $\mathrm{L}_{\mathrm{o}}$ is the deep-water wave length. The stroke limit, velocity limit, and torque limit curves are based on the maximum performance of the wave generators for a single regular wave. However, assuming that regular waves are generated for many minutes or hours of operation, as would normally be the case, the continuous operation curve is the maximum performance of the wave generators. The system limit curve is the lower limit of all of the curves, and represents the maximum regular wave height that can be continuously generated. In contrast, when generating irregular waves, the maximum wave height in the irregular wave train is not limited by the continuous operation curve. Therefore, the maximum irregular wave height increases significantly for peak wave periods ranging from $\mathrm{T}=2.0$ to $3.5 \mathrm{sec}$.

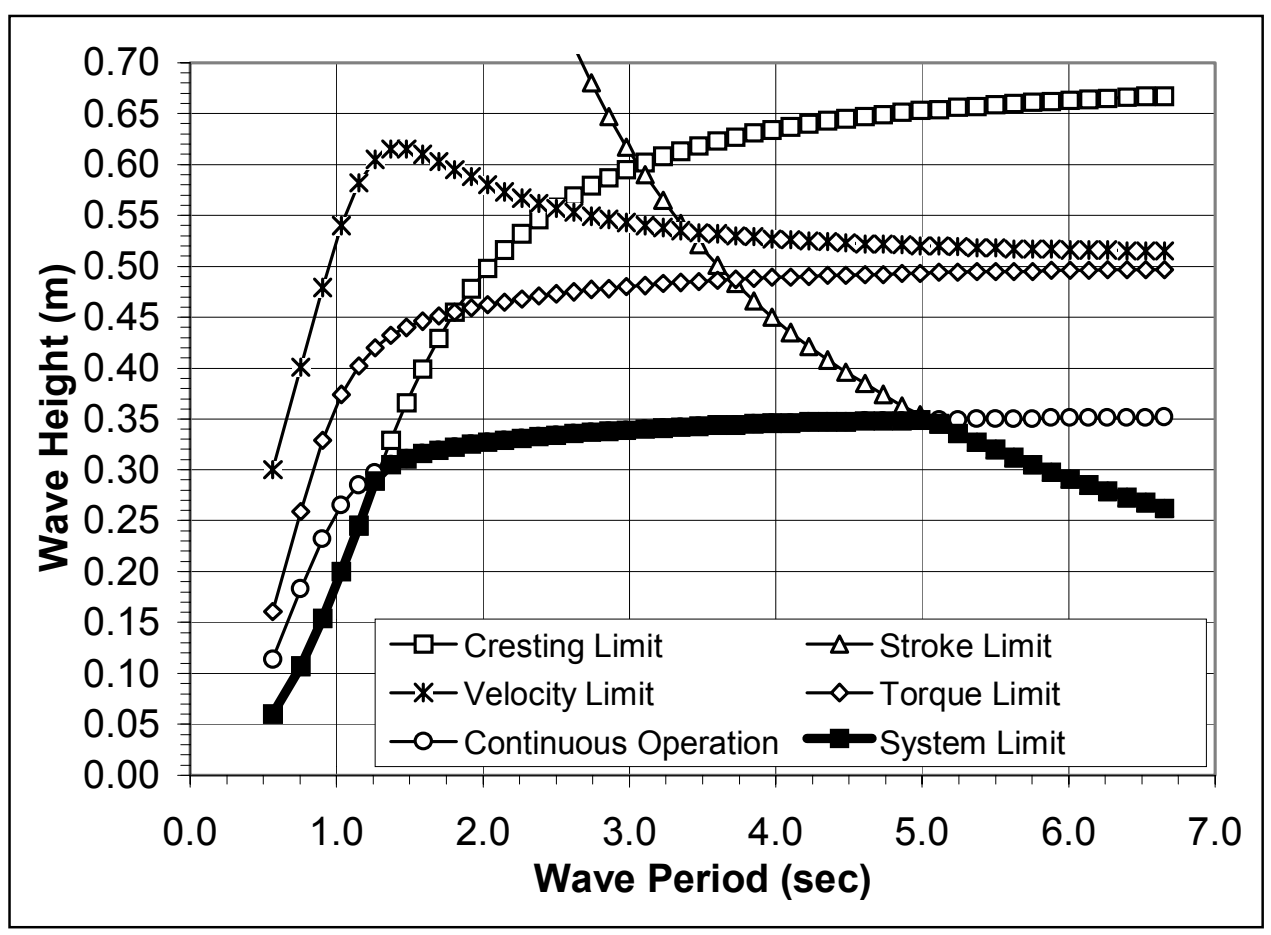

Figure 6. Performance of wave generators for a water depth of $0.9 \mathrm{~m}$

\section{Instrumentation Bridge}

A semiautomated instrumentation bridge is used to traverse the length of the testing region to allow detailed measurement of waves, currents, sediment concentration, and beach profile evolution. The bridge provides a rigid platform for mounting wave gauges, current meters, fiber-optic backscatter sensors, and a fully automated bathymetric survey system. Figure 7 is an oblique view of the downstream side of the bridge, looking in the offshore direction, during a moveable-bed experiment. For a typical experiment, wave gauges and acoustic doppler velocitimeters are co-located to provide wave and current data at the same cross-shore location. The wave gauges and current meters are separated by approximately $40 \mathrm{~cm}$, in the longshore direction, to avoid hydraulic and electrical interference between the two different types of instruments. The bridge also functions as an observation platform and provides access for personnel to manually adjust the cross-shore and vertical position of the instruments. 


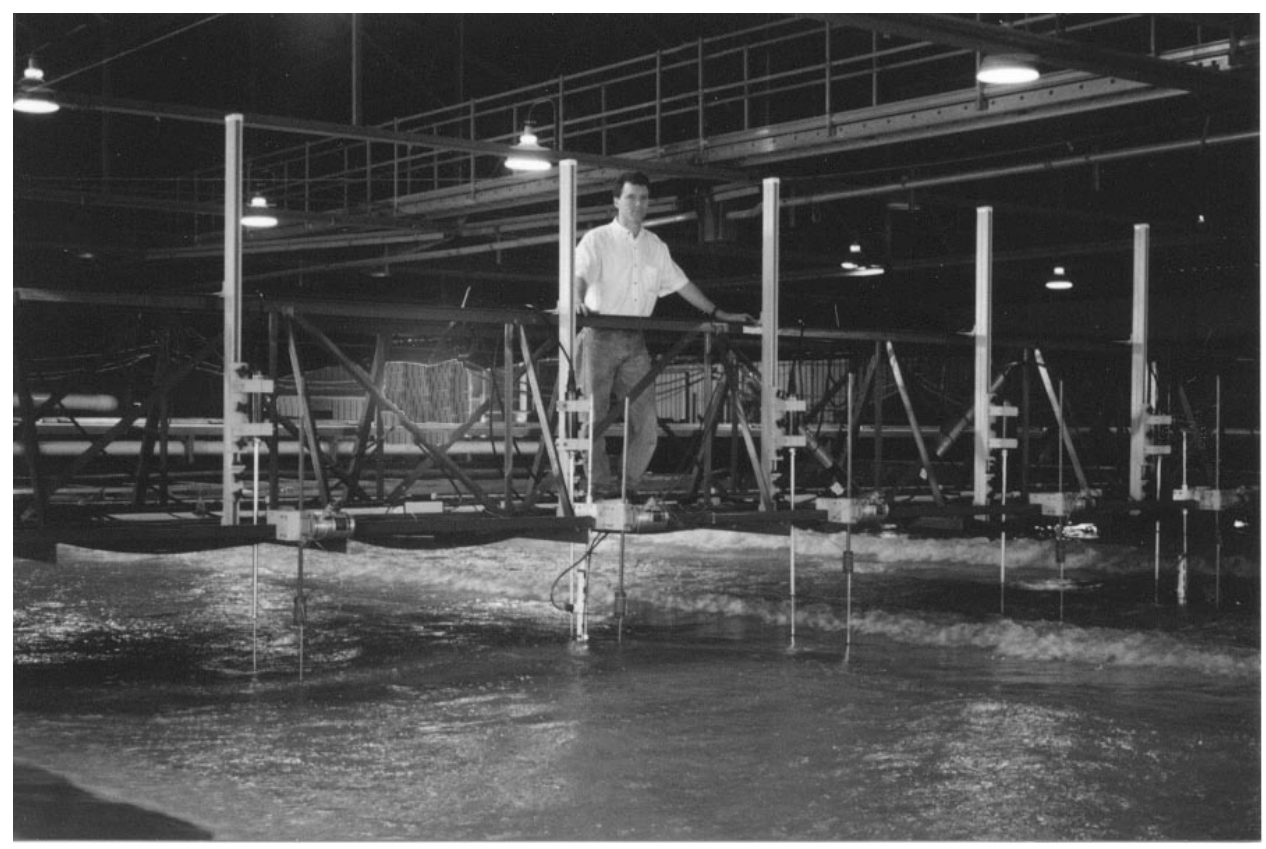

Figure 7. View of co-located sensors on instrumentation bridge

The bridge spans $21 \mathrm{~m}$ in the cross-shore direction and can traverse the entire length of the wave basin. Each end of the bridge is independently driven along support rails by a digitally controlled servo-electric drive motor. The bridge has sufficient strength to support up to $3,000 \mathrm{~kg}$ of personnel and equipment evenly distributed in the cross-shore direction.

For experiments conducted with high-water levels, the underside of the bridge is located at the nominal elevation of the top of the exterior walls of the wave basin. However, when the water level is lowered significantly, waves impact the instruments at a lower elevation resulting in increased deflections and vibrations of the instruments. To mitigate this potential problem, the bridge was designed to function at two different elevations. For experiments conducted with low-water levels the bridge can be lowered by $0.30 \mathrm{~m}$ by removing spacers located at each end of the bridge. Lowering the bridge should decrease deflections and vibrations of the sensors because the relative height of the bridge above the water level is reduced.

\section{Wave gauges}

Water surface elevation time series are simultaneously measured using 14 single-wire capacitance wave gauges. Ten of the wave gauges are mounted along the length of the instrumentation bridge to measure wave transformation (shoaling, refraction, wave breaking, and decay) from the offshore zone, across the surf zone, and into the swash zone. These wave gauges can be seen in the foreground of Figure 7 . The wave gauges can be readily repositioned at any location along the length of the bridge for various test configurations. The gauges are numbered in ascending order, starting with Wave Gauge No. 1 near the shoreline, and moving offshore to Wave Gauge No. 10. The remaining four wave gauges are fixed in an array along the $\mathrm{X}=18-\mathrm{m}$ contour line. One wave gauge is centered in front of 
each wave generator to measure the characteristics of the offshore waves. All 14 wave gauges are attached to linear actuators and can be simultaneously calibrated, as described in Chapter 8.

\section{Acoustic-doppler velocitimeters}

Orbital wave velocities and unidirectional longshore currents are measured using 10 acoustic-doppler velocitimeters (ADV). The ADVs are co-located with the wave gauges in a cross-shore array along the length of the instrumentation bridge. Each ADV is mounted to a 1.8 -m-tall vertical strut, which is mounted to the downstream side of the bridge. This allows the elevation of the ADV to be easily adjusted to locate the sampling volume at the required elevation in the water column (Figure 7). Each sensor has a 1.0-m-long stainless steel stem with a permanently engraved scale consisting of $0.1-\mathrm{m}$ major increments and $0.01-\mathrm{m}$ minor increments. Therefore, the sampling volume can be accurately positioned in the water column by using the still-water level as a reference datum. Hence, the vertical structure of the mean longshore current can be accurately measured. However, for most experiments, the ADVs are positioned so that the sampling volumes are located approximately one-third of the water depth above the bed to measure a representation of the depth-average velocity.

ADVs 3 through 10 are standard three-dimensional (3-D) down-looking sensors, which are ideal for measurements in water depths greater than $10 \mathrm{~cm}$. The down-looking sensor minimizes flow impedance when horizontal velocities are dominant. However, this probe orientation cannot be used to measure the velocity in the upper $5 \mathrm{~cm}$ of the water column. Therefore, ADVs 1 and 2 are twodimensional (2-D) side-looking sensors for measurement in shallow water close to the shoreline. The sampling volume is located $5.0 \mathrm{~cm}$ from the acoustic transmitter on all 10 systems.

\section{Fiber-optic Backscatter Sensors}

Four vertical arrays of Fiber-optic Backscatter Sensors (FOBS) are located along the length of the instrumentation bridge. Each array is co-located about midway between the wave gauges and the ADVs as shown in Figure 7. The FOBS simultaneously measure suspended sediment concentration at 19 elevations in the water column. Each of the 19 measurement locations consists of an optical fiber to transmit modulated white light into the water column and a second optical fiber to receive and transmitted light backscattered from the sand grains to a detector. Each of the four sets of sensors consists of a lower and an upper array. The lower array consists of eight emitter/receiver pairs bundled together in a circular, pipe-organ fashion with a $1.0-\mathrm{cm}$ vertical spacing. The upper array has 11 emitter/receiver pairs with a nearly logarithmic vertical spacing.

\section{Bathymetric survey system}

The moveable bed needs to be surveyed on a regular basis to quantify spatial and temporal beach profile evolution. Beach profiles are measured every $0.5 \mathrm{~m}$ 
along the beach to quantify the alongshore uniformity of the moveable bed. A fully automated 3-D bathymetric survey system was developed to minimize the time and effort required to conduct a bathymetric survey. In addition, the system needed to be amphibious so that changes to the bathymetry above the still-water level could be accurately measured.

The system consists of two primary components. First, the vertical elevation of the sand bed is measured using a commercially available beach profile indicator consisting of a mechanical spring-wheel system which tracks the elevation of the bed as the sensor moves along the length of the bridge. Figure 8 shows the profile indicator in a stationary position on the sand bed when the facility is drained. The spring-wheel assembly is located at the bottom end of the 1.52-m-long vertical rod. As the wheel rolls along the surface of the sand bed at a constant horizontal speed of $0.1 \mathrm{~m} / \mathrm{sec}$, a servomotor maintains a continuous downward force on the bed equivalent to a mass of $40 \mathrm{~g}$. An analog output voltage is provided by a potentiometer circuit, which is coupled with the sensor. This gives a vertical resolution of approximately $\pm 1 \mathrm{~mm}$. The sensor has sufficient sensitivity and frequency response to measure the sand ripples, although in some instances the wheel slightly rounds off the crest of the ripples.

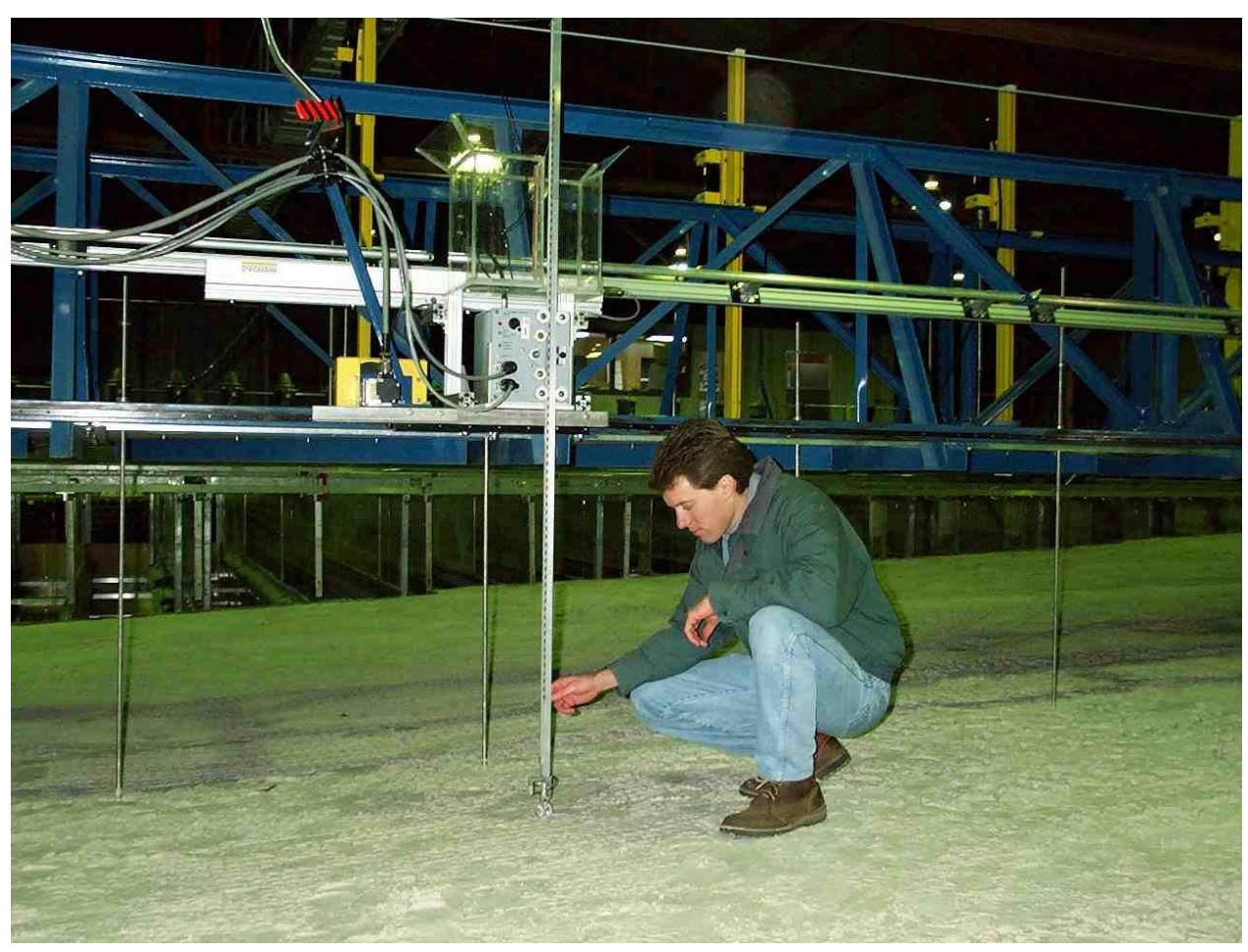

Figure 8. Beach profile indicator resting on sand beach with facility drained

The second component is a high-resolution x-y positioning system. A servocontrolled support platform moves the beach profile indicator along a linear bearing (cross-shore position) which is mounted to the updrift side of the bridge. The alongshore location of the beach profiler is controlled with the drive motors on each end of the bridge which traverse along the support rails on rigid urethane tired wheels. The position of both the $\mathrm{x}$ - and $\mathrm{y}$-axis are controlled using 
servo-electric drive motors and magnetic linear encoders. Integrating these two systems with the multichannel, closed-loop, digital control system results in a fully automated 3-D bathymetric survey system.

\section{Lateral Boundaries}

Design of the upstream and downstream lateral boundaries was a challenge, since the longshore current has to flow out of the upstream flow channels and into the downstream flow channels while wave diffraction into the flow channels needs to be minimized. Wave guides were designed to allow current to flow under the impermeable wave guide, and the height of the opening beneath each wave guide could be adjusted. The height of the opening beneath the wave guides was selected after several trial and error iterations. At the upstream boundary, a constant $0.15-\mathrm{m}$-high opening beneath the wave guide was used. At the downstream boundary, the bottom edge of the wave guide was set at approximately the minimum wave trough elevation. Therefore, at the downstream boundary, the height of the opening beneath the wave guide increased with increasing water depth. In Appendix B, Figures B-9 and B-10 show the downstream and upstream wave guides, respectively, with the facility drained. Wave guides were not used at the ends of the four most shoreward flow channels, because of the shallow-water depth and a desire to minimize obstructions to longshore sediment transport in shallow water.

In addition to the wave guides, a matrix of 0.1 -m-diam rigid polyvinyl chloride pipe was installed in the flow channels at the downstream and upstream ends of the facility. At the downstream end of the facility, the matrix of pipe was installed immediately upstream of the vertical turbine pumps to absorb the residual wave energy that entered beneath the wave guides and to minimize wave reflection in the flow channels (Appendix B, Figure B-11). At the upstream end of the facility, the matrix of pipe was installed immediately behind the wave guide to direct the discharge from the pumps to the upstream boundary of the beach (Appendix B, Figure B-10). At both ends of the facility, the matrices of pipes extended through the entire water column and across the full width of each channel. Svendsen (1991) discussed similar concepts.

\section{Control and Data Acquisition Room}

Real-time control and data acquisition of the many different types of equipment and instrumentation in the LSTF are conducted from the control room, located at the downdrift end of the facility (Figure 9). Real-time control of the wave generators and instrumentation bridge as well as data collection from the wave gauges, current meters, and the beach profile indicator are carried out using the computer located in the lower left corner of this photograph. The computer to the right is dedicated to data acquisition and real-time display of the in-line flow sensors, which measure the discharge of the pumps. The 20 flow channels and vertical turbine pumps for externally recirculating the longshore current can be seen in the foreground, through the window of the control room. The instrumentation bridge and the testing region of the facility can be seen in the upper left corner of the photograph. 


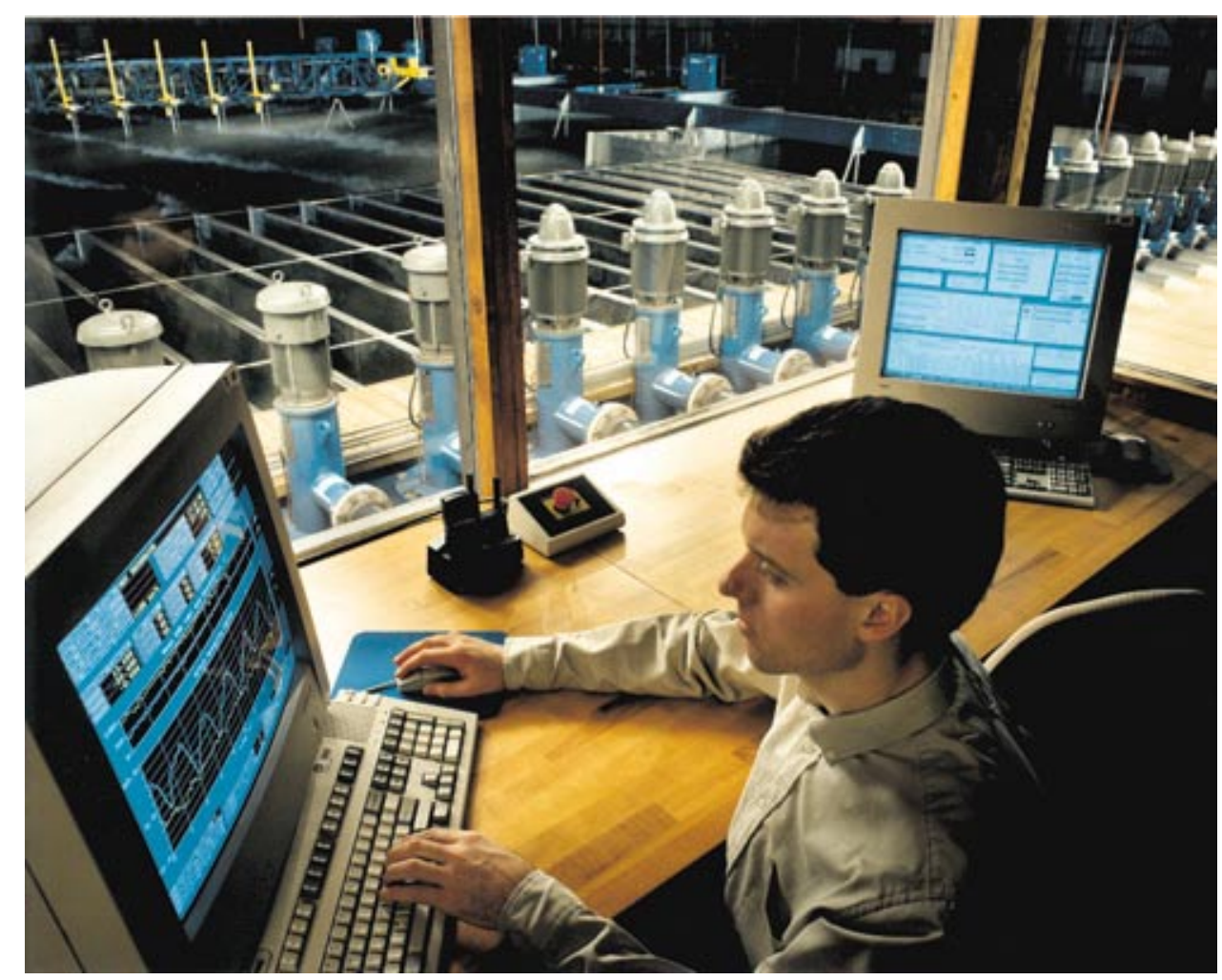

Figure 9. View of the LSTF from inside the data acquisition and control room

The LSTF is designed and constructed using state-of-the-art equipment and instrumentation. These tools provide the user with the capability to conduct accurately controlled moveable-bed laboratory experiments at a large geometric scale. Experience has shown that two operators can operate all of the equipment, instrumentation and data acquisition, and control systems simultaneously. 


\section{Longshore Current Recirculation System}

\section{Introduction}

This chapter describes the development of a large-scale longshore current (LSC) recirculation system for the LSTF. An LSC recirculation system is essentially a closed-loop system that continuously recirculates longshore current from the downdrift to the updrift lateral boundary of the beach while waves are being generated. The objective of this system is to accurately control and recirculate the wave-driven longshore current through the lateral boundaries of the facility, and therefore maximize the length of beach over which longshore uniformity of waves and wave-driven longshore currents exist in the facility. The majority of the material discussed in this chapter was published previously by Hamilton et al. (1996 and 1997).

\section{Conceptual Design}

During the past 5 decades, seven types of longshore current recirculation systems have been used to conduct laboratory investigations of longshore currents (Figure 10). The earliest systems were fairly simplistic, whereas the most recent systems are more complex and are, in general, physically larger which makes them substantially more expensive to design and construct. Each of these seven types of systems will be discussed briefly in this section. Visser (1991) and Svendsen (1991) also provide a description of several of these systems.

Type 1: Putnam, Munk, and Trayler (1949) used a completely enclosed wave basin and allowed the longshore current to recirculate in the offshore section of the wave basin.

Type 2: Galvin and Eagleson (1965) and Mizuguchi and Horikawa (1978) terminated the downstream wave guide at about the location of wave breaking, allowing the longshore flux to exit the testing region. The longshore current recirculated outside of the downdrift wave guide and reentered the testing region beneath the wave generators. 


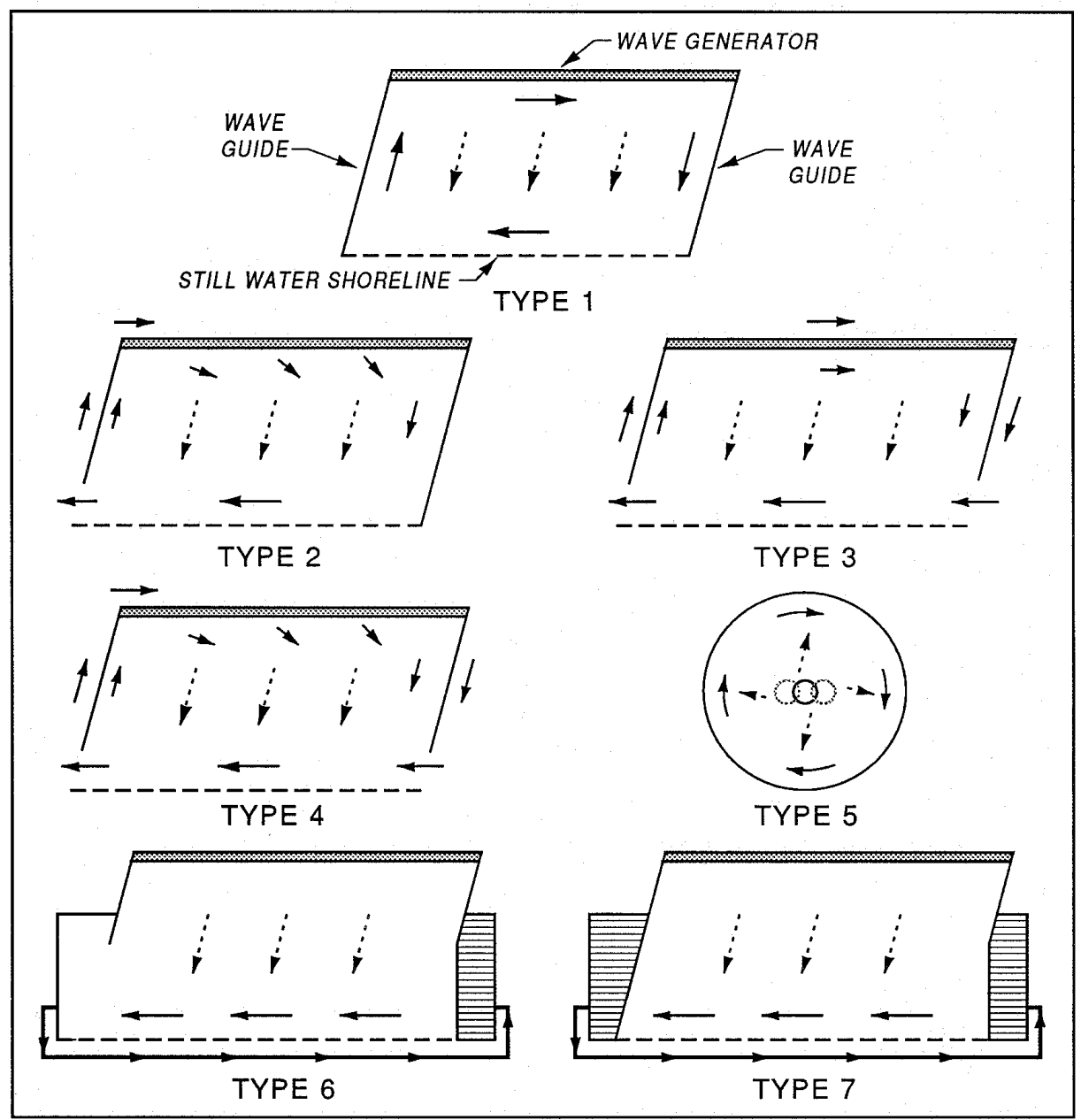

Figure 10. Seven types of longshore current recirculation systems. Illustration of Types 1 through 5 reproduced from Figure 1 (Visser 1991) (Dashed arrows denote wave direction, and solid arrows denote mean current direction)

Type 3: Brebner and Kamphuis (1963) terminated both the upstream and downstream wave guides near the breaker point. The recirculation is driven by a water level difference between the upstream and downstream ends of the facility.

Type 4: Kamphuis (1977) used a procedure almost identical to Type 3, except that the wave generators were raised slightly above the basin floor and allowed the longshore current to also reenter the testing region beneath the wave generators.

Type 5: Dalrymple and Dean (1972) used a circular wave basin with spiral wave generator and a circumferential beach. This is a unique method of avoiding the traditional boundary effects resulting from the upstream and downstream lateral boundaries of a wave basin. 
Type 6: Visser (1982 and 1991) was the first to use an active recirculation system driven by a pump. As with the Brebner and Kamphuis (1963) experiments, the wave guides were open at both ends of the beach. However, in this case, 12 independent weirs are used to input the required cross-shore distribution of longshore current at the updrift boundary.

Type 7: H. R.Wallingford (1994) and Simons et al. (1995) provide a description of the Coastal Research Facility at H.R.Wallingford, United Kingdom. This facility is a further improvement of Type 6 in that adjustable weirs are used at both ends of the facility. Therefore, the cross-shore distribution of longshore current can be controlled at both lateral boundaries.

Based on an investigation of the various techniques used to recirculate the longshore current in a wave basin with a finite length, a Type 7 recirculation system was developed for the LSTF. A Type 7 recirculation system should maximize the length of surf zone in which the hydrodynamics are essentially uniform in the alongshore direction. Furthermore, the large geometric scale of the wave conditions that can be generated in the LSTF will produce a significant amount of suspended sediment transport. It is therefore necessary to maintain relatively straight and parallel current streamlines at the downstream boundary, so that the cross-shore distribution of the total longshore sediment transport rate, (measured using sand traps at the downstream boundary) is not skewed. For this reason, the recirculation system in the LSTF was developed with the capability of controlling the cross-shore distribution of longshore current at both the upstream and downstream lateral boundaries.

\section{System Requirements}

Five primary requirements were identified for the longshore current recirculation system:

a. Must have a pumping capacity to recirculate the longshore flux associated with a wide range of wave, water level, and bathymetric conditions.

$b$. Must have the flexibility to recirculate relatively low longshore current magnitudes associated with, for example, the offshore tail of the longshore current distribution. Therefore, the system should have the capability to recirculate between 10 and 100 percent of the maximum design pumping capacity at each cross-shore location.

c. Should be capable of maintaining constant discharge rates for several hours of continuous operation to accommodate the time scales associated with conducting moveable-bed experiments at this relatively large geometric scale.

d. Should require minimal time and labor to adjust the magnitude and cross-shore distribution of the longshore current being recirculated. This is of paramount importance for moveable-bed experiments because the cross-shore distribution of recirculated longshore current must be adjusted in response to the changing beach profile. 
e. Should have a design life of 15 to 20 years given the long-term requirements of the facility.

\section{Numerical Simulation of Longshore Current}

The first part of this section provides a brief description and configuration of the numerical model used to estimate, prior to construction, the magnitude and cross-shore distribution of the longshore current that would be generated in the LSTF. The next section validates the results obtained from the numerical model using dye measurements in the LSTF. The final section shows the influence of wave height, period, and direction on the estimated magnitude and cross-shore distribution of the longshore current that would need to be recirculated.

\section{Description of the numerical model}

Kraus and Larson's (1991) numerical model NMLONG (Numerical Model of the LONGshore current) was used to estimate the wave-driven longshore current in the LSTF, prior to design and construction. NMLONG is a PC-based model that calculates cross-shore distribution pattern of wave transformation, mean water surface elevation, and longshore current. The major assumptions in NMLONG are longshore homogeneity and linear wave theory.

Using the data from Visser (1982), Kraus and Larson (1991) illustrated that NMLONG can be calibrated to reproduce the LSC measured in the laboratory with reasonable success. In particular, the magnitude and cross-shore location of the peak of the LSC distribution was reproduced with reasonable accuracy. This suggests that NMLONG can be used to provide a reasonable estimate of the magnitude and cross-shore distribution of LSC that can be generated in the LSTF. However, in the Visser cases, NMLONG predicted that the magnitude of the offshore tail of the LSC distribution was higher than measured by Visser, even after the numerical model had been calibrated.

NMLONG requires the following input parameters: offshore wave height, period, and direction, specification of regular or random waves, offshore water depth, and beach profile elevation relative to mean water level. Random waves are characterized in NMLONG using the root-mean-square wave height, $\mathrm{H}_{\text {rms }}$. Values of $\mathrm{H}_{\mathrm{rms}}$ were converted to significant wave height, $\mathrm{H}_{\mathrm{s}}$ assuming $\mathrm{H}_{\mathrm{s}}=$ $1.414 \times \mathrm{H}_{\mathrm{rms}}$. Nonlinear bottom friction with a friction coefficient equal to 0.01 was used in the LSTF simulations. For one wave condition, the sensitivity of results to this value was evaluated by reducing and increasing the coefficient to 0.005 and 0.02 , respectively. All other empirical parameters were set to the default values: incipient breaking-wave-height-to-water-depth ratio equal to 0.8 , stable wave-height-to-water-depth ratio equal to 0.4 , energy flux dissipation rate equal to 0.15 , and lateral mixing coefficient equal to 0.3 .

NMLONG was used to calculate the depth-averaged LSC velocities at each cell spaced $1.0 \mathrm{~m}$ across the beach profile. These depth-averaged velocities were multiplied by the corresponding mean water depth and integrated across the entire profile to estimate the total longshore volume flux for each wave condition. 
Two series of numerical simulations were conducted to represent two different experimental configurations planned for the LSTF. The first series was conducted with an offshore water depth equal to $0.6 \mathrm{~m}$ and represented the configuration that would be used in the LSTF during the fixed-bed hydrodynamic testing phase. The second series was conducted with a water depth equal to $0.9 \mathrm{~m}$ and represented the configuration to be used for the moveable-bed sediment transport experiments. In the later configuration, it was assumed that a $0.3-\mathrm{m}$-thick uniform layer of sand would be placed on top of the existing concrete beach. Increasing the water level from 0.6 to $0.9 \mathrm{~m}$ translates the shoreline directly upward (i.e., there is no cross-shore translation of the nearshore zone). However, the resulting magnitude and cross-shore distribution of the LSC was slightly different for the two different configurations because of the slight difference in wave transformation caused by the steeper toe of the beach in the case with the moveable bed.

Numerical simulations in both test series covered the following range of hydrodynamic conditions: significant wave height, $\mathrm{H}_{\mathrm{s}}=0.1$ to $0.4 \mathrm{~m}$, peak wave period, $\mathrm{T}_{\mathrm{p}}=1.0$ to $2.5 \mathrm{sec}$, and angle of wave incidence, $\theta=5$ to 20 deg relative to shore normal. For these test conditions, the total longshore volume flux ranged from $0.04 \mathrm{~m}^{3} / \mathrm{sec}$ for $\mathrm{H}_{\mathrm{s}}=0.1 \mathrm{~m}, \mathrm{~T}_{\mathrm{p}}=2.5 \mathrm{sec}$, and $\theta=20 \mathrm{deg}$ to 1.3 $\mathrm{m}^{3} / \mathrm{sec}$ for $\mathrm{H}_{\mathrm{s}}=0.4 \mathrm{~m}, \mathrm{~T}_{\mathrm{p}}=2.5 \mathrm{sec}$, and $\theta=20 \mathrm{deg}$.

The sensitivity of the bottom friction coefficient was evaluated for a relatively energetic wave condition; namely $\mathrm{H}_{\mathrm{s}}=0.3 \mathrm{~m}, \mathrm{~T}_{\mathrm{p}}=2.5 \mathrm{sec}$, and $\theta=20$ deg. The bottom friction coefficient was varied over a range of two times the default value of 0.01 (i.e., from 0.005 to 0.02 ). Results obtained using the lower friction coefficient (0.005) resulted in a total longshore volume flux of 1.1 and $1.2 \mathrm{~m}^{3} / \mathrm{sec}$ for the 0.6 and $0.9 \mathrm{~m}$ water-depth cases, respectively. The higher friction coefficient $(0.02)$ reduced the total longshore volume flux to $0.4 \mathrm{~m}^{3} / \mathrm{sec}$ for both water-depth cases. Therefore, the results obtained using NMLONG to predict the total longshore volume flux in the LSTF are sensitive to the value of the bottom friction coefficient.

\section{Validation using dye measurements}

Preliminary measurements of the magnitude of the longshore current generated in the LSTF were obtained by injecting dye into the surf zone while generating monochromatic waves. These measurements then were used to assess the validity of the numerical simulations. However, the following facility components had not been installed in the LSTF at the time these measurements were required:

a. Lateral wave guides were not available to direct the incident waves from the wave generators to the surf zone.

b. None of the longshore current recirculation system components (i.e., pumps and flow channels) were available to minimize the laboratory effects caused by the lateral boundaries of the facility. 
c. Only monochromatic waves could be generated with the wave generators, because the software for synchronizing the wave generators for irregular waves was still in development.

As a result, strong adverse reflection patterns (from the vertical walls at the two ends of the basin) and circulation cells developed throughout the facility during the first 5 to 10 generated waves. In addition, no flow velocity measurement sensors were available to accurately measure the wave-driven longshore current in the surf zone.

Three conclusions were made based on the limited dye measurements. First, NMLONG produced reasonable estimates of the peak longshore current magnitude and cross-shore location, using the default bottom friction coefficient of 0.01 . However, insufficient data were collected to calibrate this coefficient. Secondly, we were unable to verify the cross-shore distribution of the longshore current predicted using NMLONG because of the physical limitations and resulting adverse laboratory effects previously discussed. Therefore, the default lateral mixing coefficient (0.30) was used. Thirdly, observations made during the dye experiment strongly reinforced the expectation that a properly designed external longshore current recirculation system would be required to maintain longshore uniformity of waves and wave-driven currents in the facility, especially for energetic wave conditions.

\section{Influence of wave height}

Figure 11 shows the calculated LSC distribution for $\mathrm{H}_{\mathrm{s}}=0.2,0.3$, and $0.4 \mathrm{~m}$, with $\mathrm{T}_{\mathrm{p}}=2.5 \mathrm{sec}$ and $\theta=20 \mathrm{deg}$ at the wave generator. Three general trends can be seen in this figure. First, as $\mathrm{H}_{\mathrm{s}}$ increases, the magnitude of the LSC at the peak of the distribution significantly increases. The magnitude of the peak LSC equals $0.21,0.28$ and $0.36 \mathrm{~m} / \mathrm{sec}$ for $\mathrm{H}_{\mathrm{s}}=0.2,0.3$ and $0.4 \mathrm{~m}$, respectively. This is a relative increase of approximately 30 percent for each $0.1-\mathrm{m}$ increase in wave height in these cases. Secondly, the peak of the LSC distribution moves offshore as $\mathrm{H}_{\mathrm{s}}$ increases, because incident waves begin to break farther offshore. Thirdly, the width of the LSC distribution increases as $\mathrm{H}_{\mathrm{s}}$ increases, because the width of the surf zone increases.

\section{Influence of wave period}

Figure 12 shows the LSC distribution for $\mathrm{T}_{\mathrm{p}}=1.0,1.5,2.0$, and $2.5 \mathrm{sec}$ with $\mathrm{H}_{\mathrm{s}}=0.3 \mathrm{~m}$ and $\theta=20 \mathrm{deg}$ at the wave generator. Three general trends can be seen in this figure. First, as $\mathrm{T}_{\mathrm{p}}$ increases, the magnitude of the LSC at the peak of the distribution increases slightly. The magnitude of the peak LSC equals 0.25 , $0.26,0.27$, and $0.28 \mathrm{~m} / \mathrm{sec}$ for $\mathrm{T}_{\mathrm{p}}=1.0,1.5,2.0$, and $2.5 \mathrm{sec}$, respectively. This is a relative increase of only 4 percent for each 0.5 -sec increase in $T_{p}$ for these cases. Secondly, the peak of the LSC distribution moves slightly farther offshore as $\mathrm{T}_{\mathrm{p}}$ increases, because incipient breaking occurs slightly farther offshore. Thirdly, the width of the LSC distribution increases slightly as $T_{p}$ increases, because the width of the surf-zone increases slightly. 


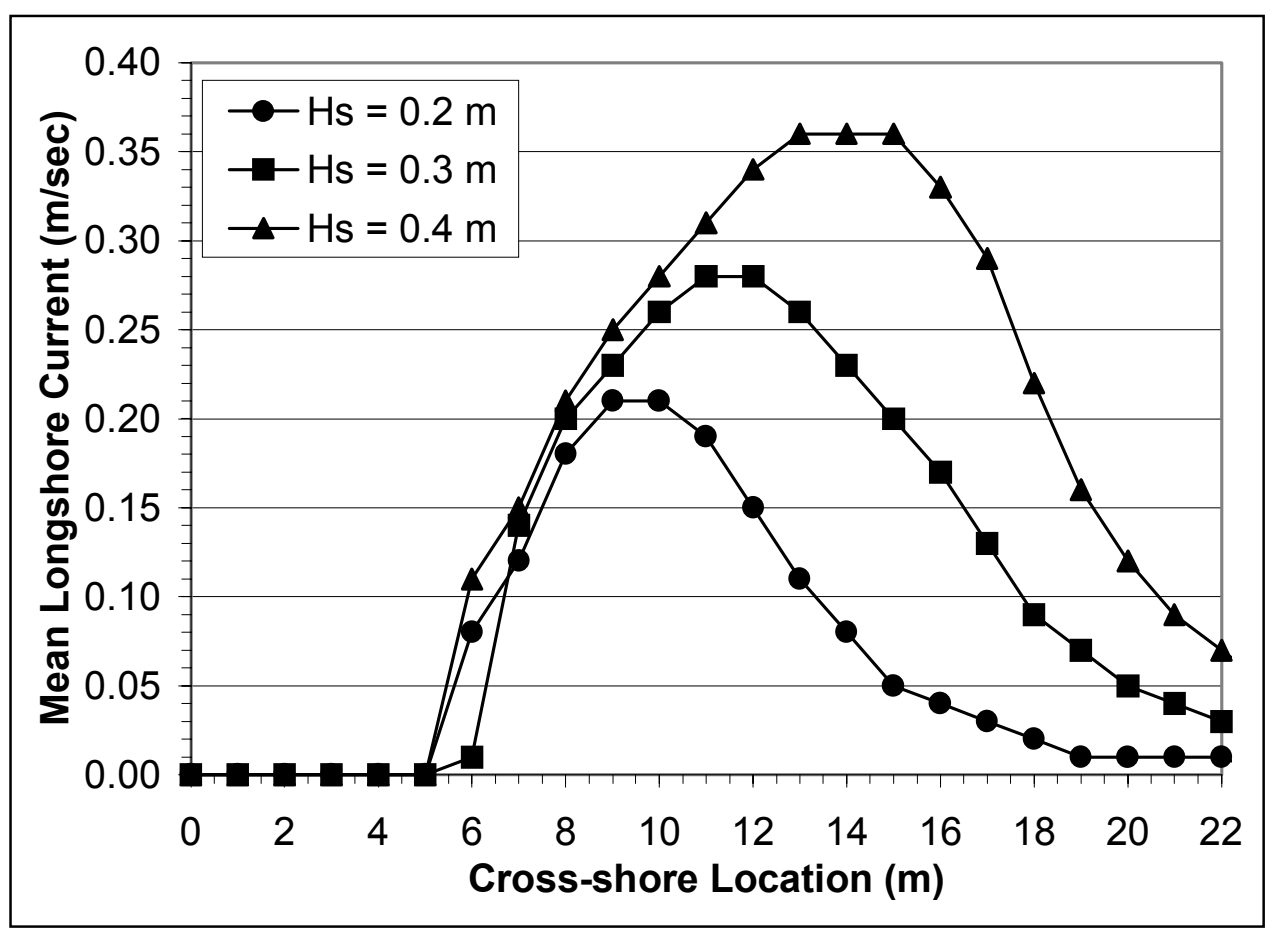

Figure 11. Influence of wave height on required longshore current distribution

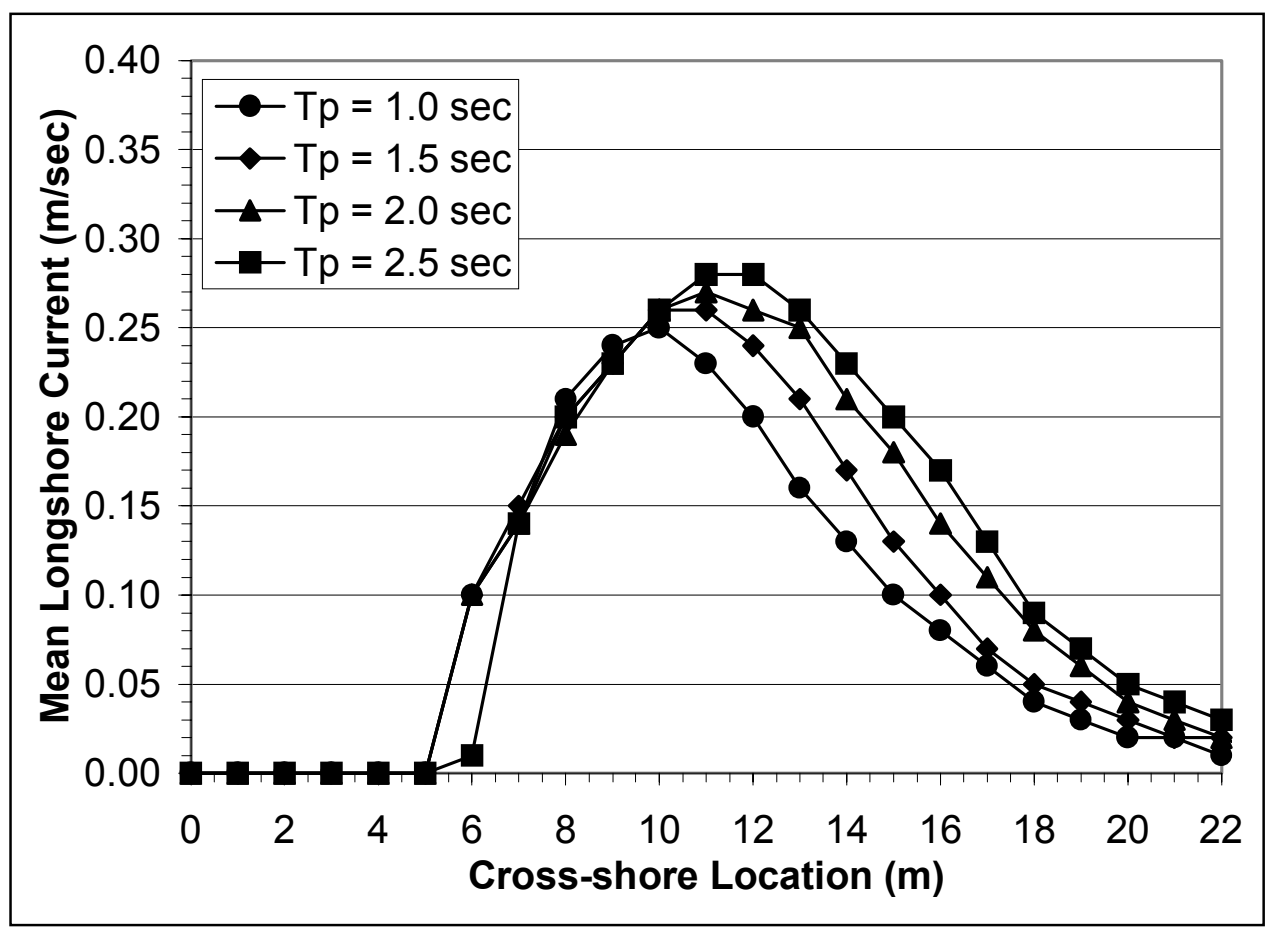

Figure 12. Influence of wave period on required longshore current distribution 


\section{Influence of incident wave direction}

Figure 13 shows the LSC distribution for $\theta=5,10,15$, and 20 deg with $\mathrm{H}_{\mathrm{s}}=0.3 \mathrm{~m}$ and $\mathrm{T}_{\mathrm{p}}=2.5 \mathrm{sec}$. Three general trends can be seen in this figure. First, as $\theta$ increases, the magnitude of the LSC at the peak of the distribution significantly increases. The magnitude of the peak LSC equals $0.10,0.18,0.23$, and $0.28 \mathrm{~m} / \mathrm{sec}$ for $\theta=5,10,15$, and $20 \mathrm{deg}$, respectively. This is a relative increase of about 80, 28, and 22 percent for each consecutive increase in $\theta$. Secondly, the cross-shore location of the peak of the LSC distribution remains constant as $\theta$ increases. Thirdly, the width of the LSC distribution remains reasonably constant as $\theta$ increases.

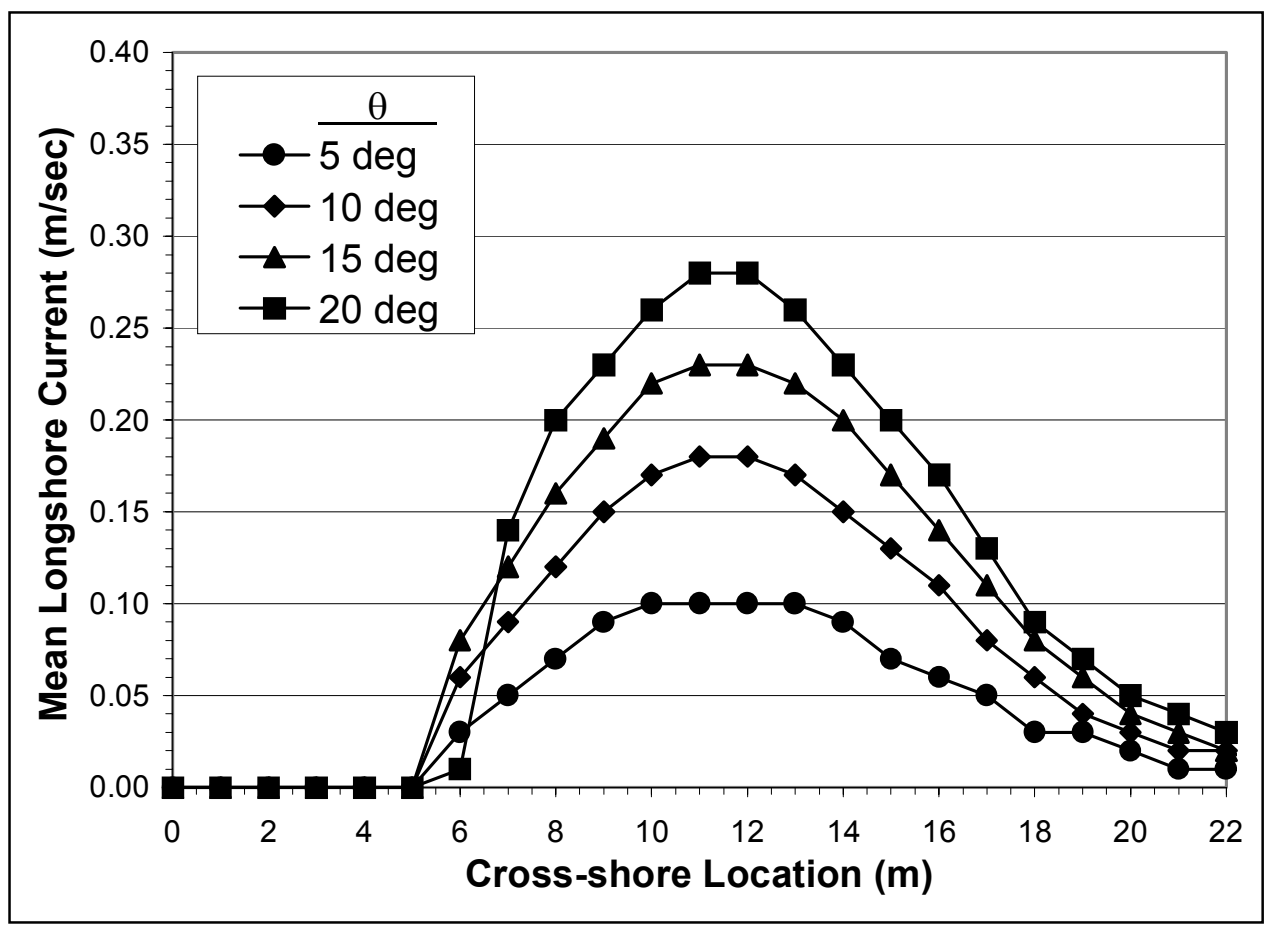

Figure 13. Influence of wave direction on required longshore current distribution

Based on these numerical simulations, it was concluded that increasing $\mathrm{H}_{\mathrm{s}}$ strongly influences the magnitude and cross-shore location of the peak of the LSC distribution, whereas, increasing $T_{p}$ has a much milder effect. In addition, increasing $\theta$ has a very strong influence on increasing the peak magnitude but no influence on the cross-shore location of the peak of the LSC distribution.

\section{Design Parameters}

The first part of this section describes the design wave condition used to determine the required pumping capacity of the LSC recirculation system. Subsequent sections apply a factor of safety to the numerical model results, and incorporate the requirements of conducting experiments with a moveable-bed beach and a variable operating water level into the design. 


\section{Design wave condition}

To determine the design capacity of a LSC recirculation system for the LSTF, a maximum design wave condition was selected. After observing the location of the break point for a number of different wave height and period combinations in the LSTF (and comparing these results with the numerical simulations), it was decided that $H_{s}=0.3 \mathrm{~m}$ and $T_{p}=2.5 \mathrm{sec}$ is probably the most energetic wave condition that will be generated during the moveable-bed LST experiments. As mentioned previously, the maximum angle-of-wave incidence (measured at the wave generators) to be tested in the LSTF is $20 \mathrm{deg}$. Therefore, the "design wave condition" used to determine the maximum required pumping capacity of the LSC recirculation system was characterized by $\mathrm{H}_{\mathrm{s}}=0.30 \mathrm{~m}$, $\mathrm{T}_{\mathrm{p}}=2.5 \mathrm{sec}$, and $\theta=20 \mathrm{deg}$ with an offshore water depth of $0.9 \mathrm{~m}$. This is the LSC distribution shown previously in Figure 11 (middle curve) and Figures 12 and 13 (upper curve). Results obtained using NMLONG to predict the magnitude and cross-shore distribution of LSC for this "design wave condition" indicated a total longshore flux of $0.76 \mathrm{~m}^{3} / \mathrm{sec}$ within the surf zone.

\section{Determining a factor of safety}

A factor of safety was incorporated into the design to compensate for inaccuracies in calculating the magnitude and cross-shore distribution of LSC that will be generated in the LSTF. Our concerns included: (a) having inadequate data to calibrate the bottom friction coefficient and the lateral mixing coefficient in the numerical model; and (b) changes in the bottom roughness between the initial hydrodynamic tests on a fixed-bed concrete beach and multiple grain sizes used for the moveable-bed beach for the LST experiments. The surface of the concrete beach was broom-finished to simulate the roughness of a course grained sand. However, because bed-forms would develop in the LST experiments, it was assumed that the moveable-bed beach would have a higher friction coefficient for a given wave condition. Because of these uncertainties, the magnitude of the LSC at each cross-shore location was increased by 10 percent (i.e., a Factor of Safety =1.1) for the purpose of determining the required pumping capacity of the LSC recirculation system.

\section{Allowance for maximum depth of erosion}

At this point in the design process, predictions of the LSC magnitude and cross-shore distribution to be generated in the LSTF were based on a 1:30 plane sloping fixed-bed beach having parallel contours. However, for future moveablebed experiments, the beach profile would adjust in response to the incident wave conditions, creating a shore-parallel bar and trough feature near the location of initial wave breaking. Assuming longshore uniformity in the beach bathymetry, the capacity of the LSC recirculation system had to be increased wherever the beach profile would erode, because of the increased longshore flux at that crossshore location.

To investigate this problem, 2-D flume tests were conducted to estimate the maximum depth of erosion during moveable-bed LST experiments in the LSTF. 
An initial 1:30 plane-sloping beach was constructed using sand having $\mathrm{D}_{50}=$ $0.11 \mathrm{~mm}$, which was the same initial beach slope and sediment size to be used for the fine-grained moveable-bed test series in the LSTF. In each experiment, the maximum depth of erosion was located just offshore of the still-water shoreline. For the design wave condition, the maximum depth of erosion did not exceed $0.1 \mathrm{~m}$; however, this conclusion is based on 2-D flume tests, and not 3-D tests in the LSTF.

For design purposes, it was assumed that the entire 18-m width of the moveable-bed beach would erode by $0.1 \mathrm{~m}$. Although this was only a rough assumption, it was conservative in that it allowed for the maximum depth of erosion to occur at any location across the beach profile. In particular, this was a very conservative assumption near the offshore end of the beach where accretion would occur (as opposed to erosion) as the offshore bar developed. Nonetheless, over designing the LSC recirculation system near the offshore region of the beach would give the system more flexibility in the future when conducting moveable-bed experiments with coastal structures.

Note that this analysis assumed that as the offshore bar and trough feature developed in the moveable-bed experiments, the depth-averaged LSC at any cross-shore location would not change substantially from the case with a plane sloping fixed-bed beach. Although this may not be the case, it was assumed that as the beach profile developed changes to the depth-averaged LSC would be relatively small, since the initial beach slope was relatively gentle (1:30) and the total profile adjustment would be relatively small. Therefore, this assumption would be adequate for design purposes.

\section{Allowance for variable water level}

The design of the LSC recirculation system also accounted for the requirement to conduct experiments with variable water levels in the facility. As mentioned previously, the plan was to conduct the majority of the moveable-bed LST experiments with an offshore water depth of $0.9 \mathrm{~m}$. However, if the water level is increased in the future, the LSC distribution would need to be translated shoreward.

A number of physical constraints in the vertical dimension of the facility dictated that the maximum operating water level could not exceed $1.0 \mathrm{~m}$ (at least for energetic wave conditions). Lower water levels could be used, however, the wave generation capability of the wave makers would decrease with decreasing water level. Therefore, for design purposes, the critical design parameter was the maximum operating water level. The first curve in Figure 14 shows the longshore flux distribution for the design wave condition, with an offshore water depth of $0.9 \mathrm{~m}$. This curve includes the increased capacity required by using a factor of safety of 1.1 and the assumption that the moveable bed eroded $0.1 \mathrm{~m}$ across the entire width of the beach. The abrupt change in cross-shore gradient at $\mathrm{X}=18$ and $21 \mathrm{~m}$ was caused by the sudden change in beach slope at these locations. The second curve was obtained by increasing the offshore water depth to $1.0 \mathrm{~m}$ for the same design wave condition. Essentially, this $0.1-\mathrm{m}$ increase in operating water level translates the LSC distribution $3.0 \mathrm{~m}$ shoreward, because 
the slope of the concrete beach is 1:30. The third curve in the figure encompasses both the first and second curves and represents the required pumping capacity of the LSC recirculation system for water levels ranging from 0.9 to $1.0 \mathrm{~m}$. As shown in the figure, incorporating a variable water level into the design essentially broadens the width of the LSC distribution that needs to be recirculated within the facility.

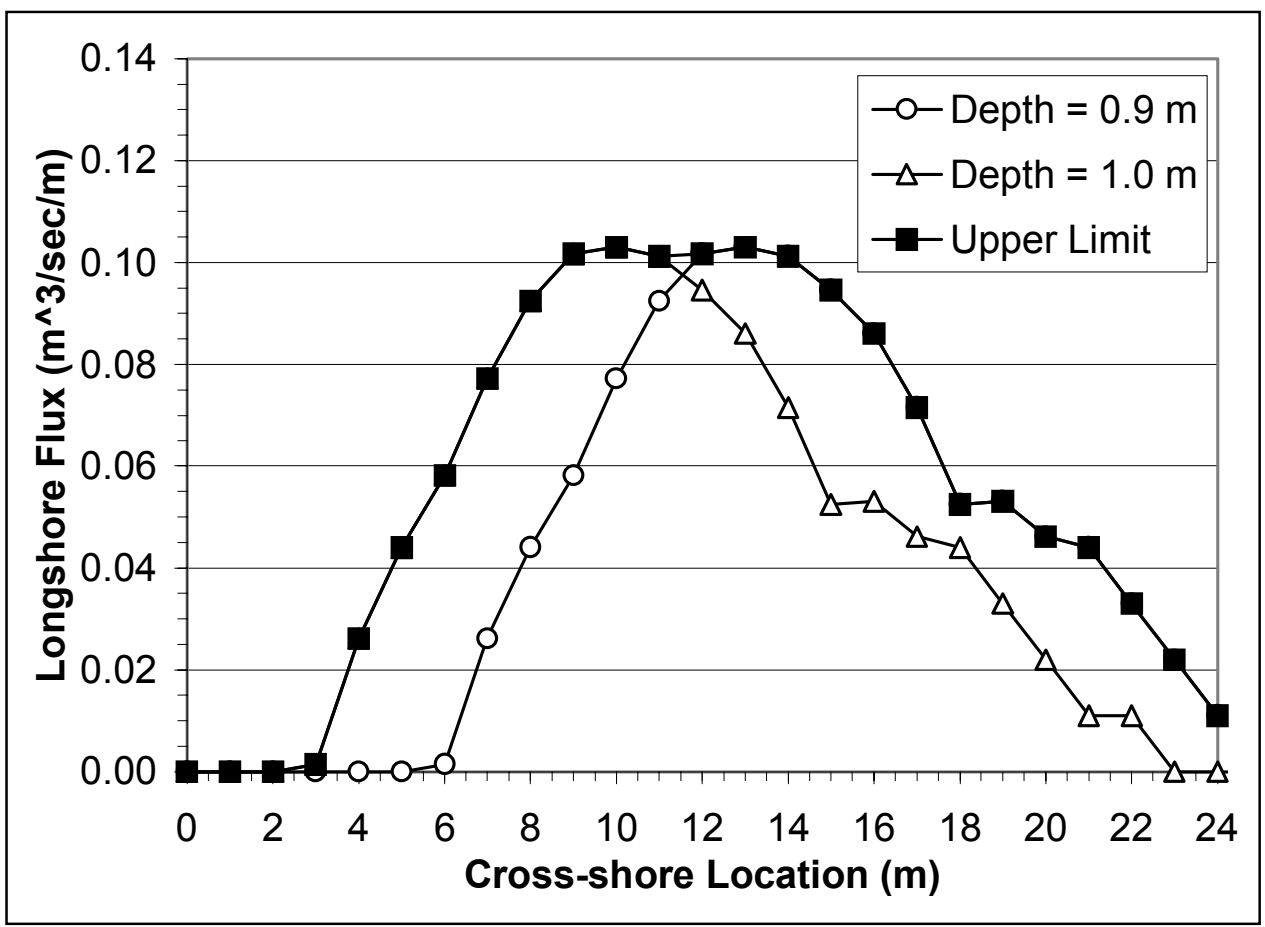

Figure 14. Influence of water depth on required longshore current distribution

\section{Design Pumping Capacity}

The last step in this phase of the design process was to determine the design capacity of each of the individual pumps that make up the LSC recirculation system. Required pump capacity is a function of the width of the flow channels at the downdrift end of the facility, which is directly related to the cross-shore resolution of the LSC recirculation system. Intuitively, one would expect that the higher the cross-shore resolution, the longer the "testing region" where longshore uniformity of waves and wave-driven longshore currents exist. After reviewing the designs of LSC recirculation systems used in other laboratory facilities, it was decided that the flow channels at both ends of the LSTF should be $0.75 \mathrm{~m}$ in width. This was a compromise between cross-shore resolution and the estimated cost of construction.

Knowing the required pumping capacity of the LSC recirculation system, shown as the upper limit in Figure 14, and given a flow channel width of $0.75 \mathrm{~m}$, the capacity of each individual pump could be readily calculated. Figure 15 shows a bar graph expressed in litres/sec for every $0.75-\mathrm{m}$ width of surf zone, and the magnitude of each bar represents the required pumping capacity of each individual pump. To reduce the number of pumps near the shoreline, where the 
longshore flux was estimated to be relatively small, one larger pump was used in place of four smaller pumps. The required pump capacities (based on the numerical simulations) and design pump capacities (as-built) are slightly different because of the need to minimize cost by selecting pumps with capacities which were commercially available. In addition, it was prudent to have additional pumping capacity at the offshore of the system or the result of uncertainties in estimating the longshore current magnitude in that region.

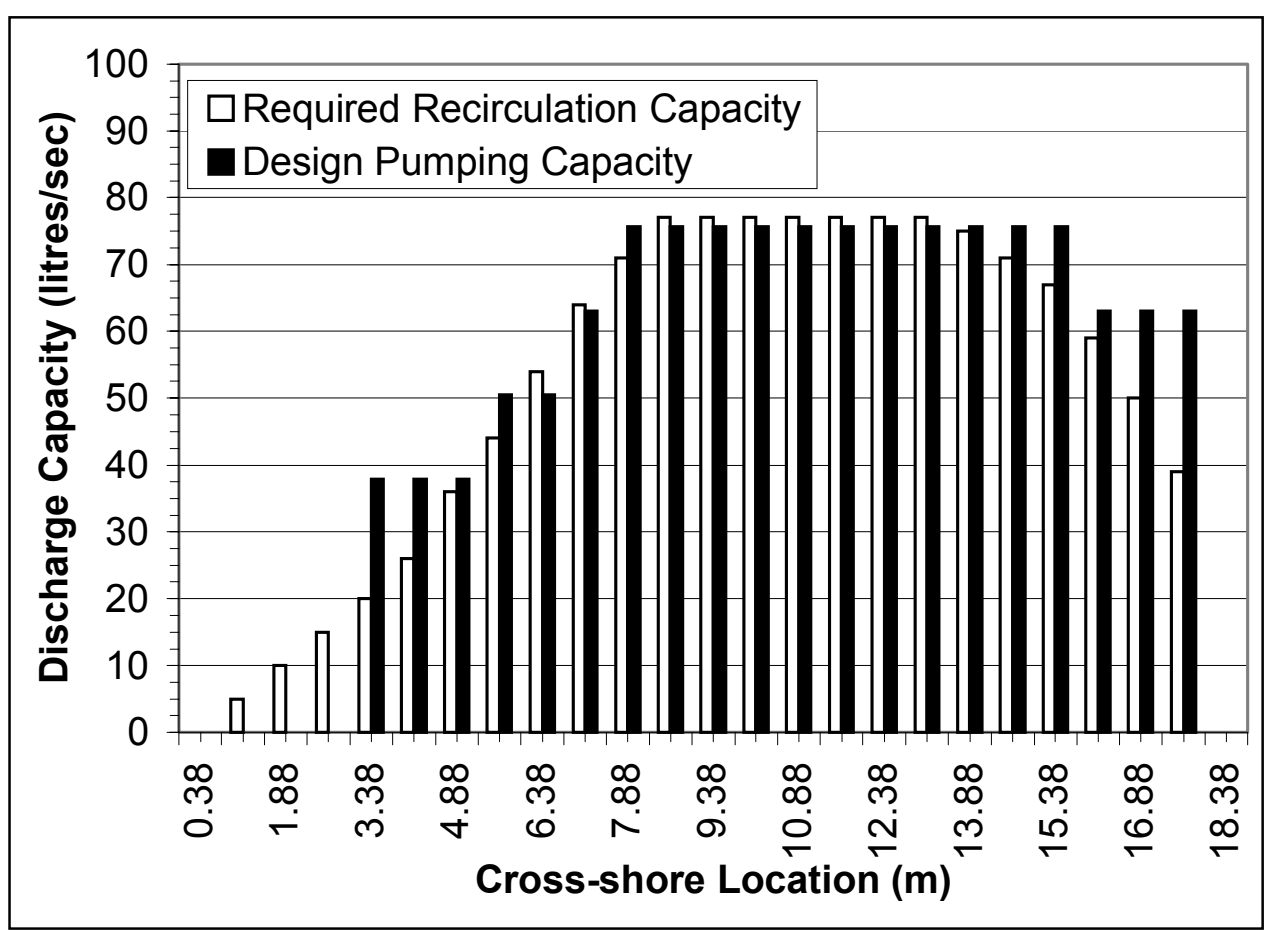

Figure 15. Required and design pumping capacity of the longshore current recirculation system

The total design capacity of the LSC recirculation system was calculated to be approximately $1.25 \mathrm{~m}^{3} / \mathrm{sec}$. To put this pumping capacity into perspective with other large-scale laboratory facilities, HR Wallingford (1994) states that the LSC recirculation system in the Coastal Research Facility has a design capacity of $1.2 \mathrm{~m}^{3} / \mathrm{sec}$ (i.e., four axial flow pumps each with a capacity of $0.3 \mathrm{~m}^{3} / \mathrm{sec}$ ). The LSC recirculation system in the Coastal Research Facility is used to generate not only wave-driven LSC within the surf zone, but also tidal currents offshore of the toe of the concrete beach. Therefore, although the purpose of the two recirculation systems is not exactly the same, the total design pumping capacities are very similar.

\section{Design of System Components}

This section provides a detailed technical description of each of the five subsystems that make up each of the 20 independent recirculation systems. 


\section{Vertical turbine pumps and motors}

Vertical turbine pumps were chosen for this application based on a desire to alleviate problems with priming and air entrainment. These problems are completely eliminated since, by definition, the impeller on a vertical turbine pump is located below the mean water level.

Figure 16 provides an oblique view of the 20 vertical turbine pumps, drive motors, and discharge pipes immediately after the installation was complete. Each vertical turbine pump consists of a single-stage impeller and bowl assembly, a vertical column assembly, a discharge head, and an electric drive motor. Closed-type Francis-Vane impellers were used to eliminate the requirement of having to adjust the clearance beneath the impellers to maintain optimum hydraulic efficiency. The impellers are constructed of high-strength silicon bronze and the suction bowls have vitra glass-lined waterways for maximize abrasion resistance. Minimizing long-term abrasion in the pumps was an important design criterion, because it was anticipated that small quantities of sand would pass through the systems during moveable-bed experiments.

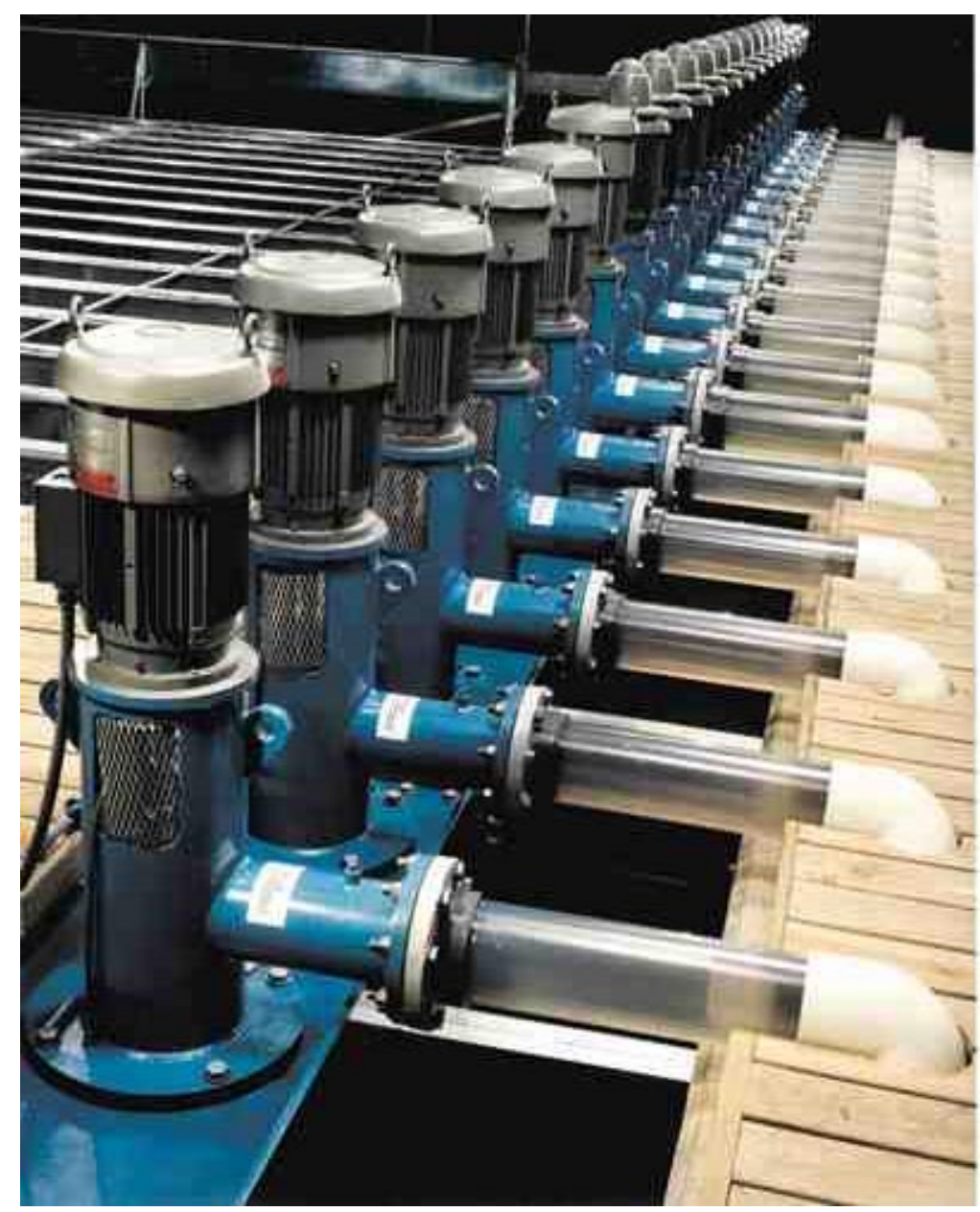

Figure 16. Oblique view of 20 vertical turbine pumps and motors 
Table 1 lists the primary design specifications of each pump including: discharge capacity at the best efficiency point; total dynamic discharge head; discharge pipe diameter; impeller diameter, optimized to the best efficiency point of each pump; hydraulic efficiency; specific speed; and the electric power required to drive each pump at maximum discharge. The specific speed of all four models of pumps ranged from 2,880 to 4,340 . Hence, each of the pumps can be classified as mixed-flow pumps, which generate discharge head through a combination of centrifugal and axial forces. The electric motors are inverter duty motors manufactured using special inverter grade insulation so that the motors do not overheat during low-speed operation. Pump No. 1 is located at the onshore end and Pump No. 20 is at the offshore end of the recirculation system. For additional information on the drive motors for the vertical turbine pumps, see U.S. Motors (1996).

\begin{tabular}{|c|c|c|c|c|c|c|c|}
\hline $\begin{array}{l}\text { Pump } \\
\text { Location } \\
\text { No. }\end{array}$ & $\begin{array}{l}\text { Discharge } \\
\text { Capacity } \\
\text { e/sec }\end{array}$ & $\begin{array}{l}\text { Total } \\
\text { Dynamic } \\
\text { head } \\
\mathbf{m} \\
\end{array}$ & $\begin{array}{l}\text { Discharge } \\
\text { Pipe } \\
\text { Diameter } \\
\text { mm } \\
\end{array}$ & $\begin{array}{l}\text { Optimum } \\
\text { Impeller } \\
\text { Diameter } \\
\text { mm }\end{array}$ & $\begin{array}{l}\text { Hydraulic } \\
\text { Efficiency } \\
1,800 \text { RPM } \\
\% \\
\end{array}$ & $\begin{array}{l}\text { Specific } \\
\text { Speed }\end{array}$ & $\begin{array}{l}\text { Required } \\
\text { Power } \\
\text { kW }\end{array}$ \\
\hline $1-3$ & 37.8 & 9.8 & 150 & 149 & 79 & 2,880 & 4.6 \\
\hline 4,5 & 50.5 & 12.2 & 150 & 176 & 81 & 2,580 & 7.4 \\
\hline $6,18-20$ & 63.1 & 8.8 & 200 & 149 & 79 & 4,340 & 6.9 \\
\hline $7-17$ & 75.7 & 11.9 & 200 & 178 & 80 & 3,930 & 11.0 \\
\hline
\end{tabular}

\section{Variable speed motor controllers}

To meet the design requirement that each pump-and-piping system should have the capability to recirculate between 10 and 100 percent of the maximum design discharge, variable speed motors were used to drive the pumps. It was concluded that throttling valves, weirs, or other mechanical control mechanisms are too labor intensive to operate because there are 20 independent pump-andpiping systems. Variable speed motors were the most desirable solution for two primary reasons: (a) there is a linear relationship between flow rate and pump speed giving accurate control of discharge over a wide range of flow rates; and (b) pump discharge rates could be remotely controlled and adjusted with a minimal amount of time and effort. As a result, 20 pulse-width-modulated, variable-frequency, alternating-current motor controllers were used to control the speed of the pumps. Eleven of the motor controllers are $11.25-\mathrm{kW}$ systems and nine are $7.5-\mathrm{kW}$ systems. The output frequency of the motor controllers, and hence the speed of the pumps, can be manually adjusted using a human interface key pad, with digital display, mounted on the front panel of each controller (Figure 17). Electrical power is provided by a 400 -ampere, 480 -volt, 3-phase, $60-\mathrm{Hz}$, electrical service. 


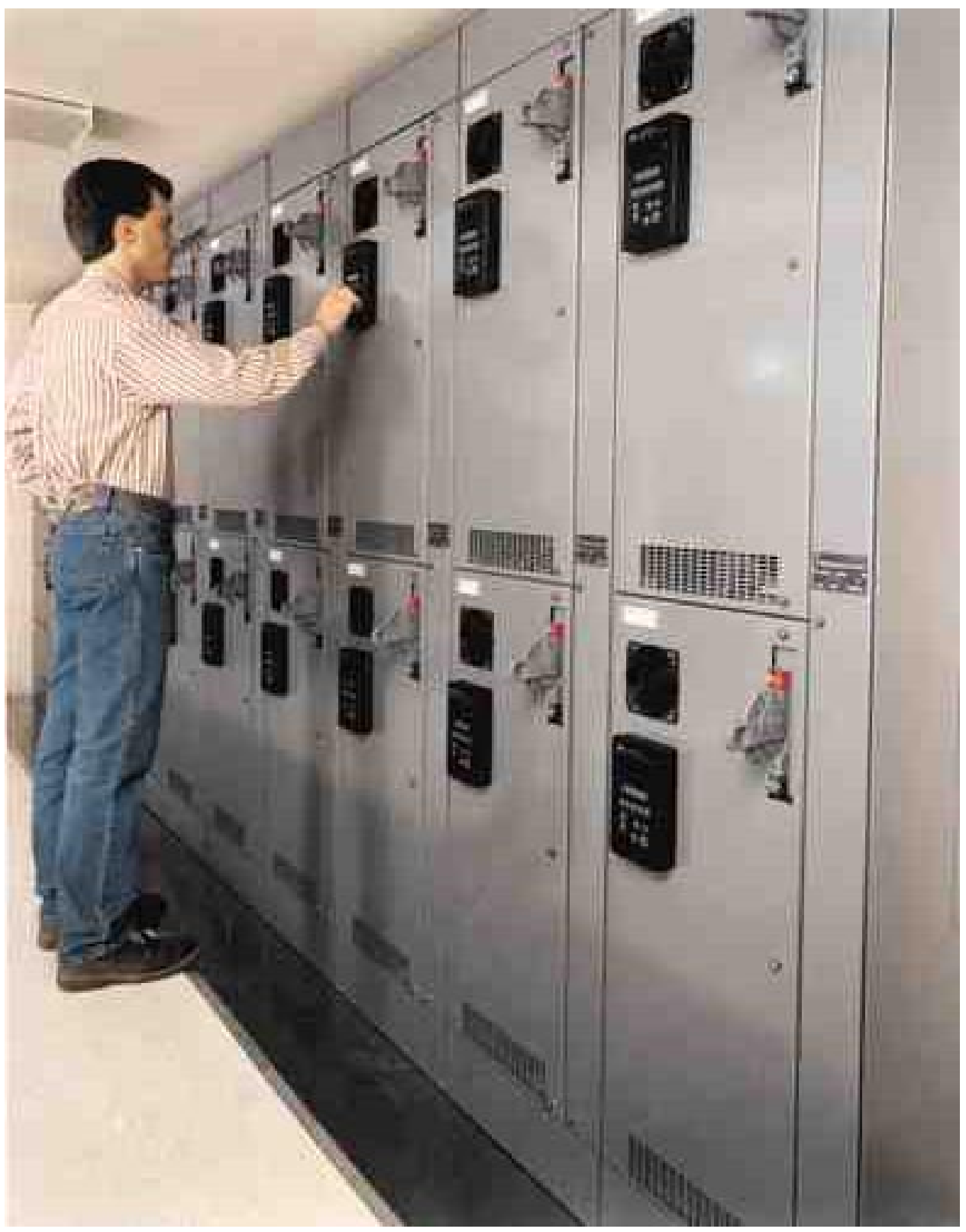

Figure 17. Variable speed motor controller with digital human interface

\section{Piping system}

The piping system consists of fifteen 200 -mm- and five 150-mm-diam pipelines, with a total pipe length of approximately $1,500 \mathrm{~m}$. Selection of the discharge pipe diameter was based primarily on economics. The cost of various pipe sizes was compared with the associated pump and motor size required to overcome the resulting head loss through the piping system at maximum discharge. All of the pipe, fittings, and valves are constructed of rigid polyvinyl chloride. The advantages of polyvinyl chloride are that it has good pressurebearing capability, exceptional long-term corrosion resistance, and smooth 
interior walls to minimize head loss. Transparent polyvinyl chloride pipe was used at the pump discharge heads and immediately upstream of the flow sensors as observation windows to determine if air or sediment is being pumped through the piping system. This may be monitored because the pumps are designed to withstand only small quantities of sand passing though the system and because the flow sensors, discussed in the next section, are somewhat sensitive to air and sediment entrainment.

\section{Flow measurement system}

Midway along each pump-and-piping system, the main pipeline branches out into two smaller pipelines (total of 40) running parallel for approximately $4.0 \mathrm{~m}$ before converging back into the main pipelines. These smaller pipelines are the in-line flow measurement sections and each contains a transparent section of polyvinyl pipe used for monitoring of air and sediment entrainment, a true union ball valve, which is either completely closed or completely open, and an impeller-type flow sensor. Systems No. 1, 2, and 3 have 38- and 75-mm-diam flow measurement sections, whereas System No. 4 through 20 have 50- and 100-mm-diam flow measurement sections. Figure B-7, Appendix B, shows one of the parallel flow measurement systems prior to installation into the longshore current recirculation system. There are a total of 20 of these systems, one for each pump-and-piping system. For high-flow conditions in a given system, the valve in the smaller pipe is closed and the flow rate is measured using the flow sensor in the larger of the two parallel pipes. In contrast, for low flow conditions, the ball valve in the larger pipe is closed and the flow rate is measured using the flow sensor in the smaller pipe. None of the valves are used to throttle the flow rate; they are either completely closed or fully open. The reasons for using two parallel pipelines with individual flow sensors were two fold: (a) to increase the water velocity at the flow sensor during low-flow conditions, to minimize measurement error; and (b) to increase the range of discharge rates over which the vertical turbine pumps will operate (Section entitled "Hydraulic Characteristic Curves" in Chapter 3).

Each of the flow sensors has a six-blade impeller with a nonmagnetic sensing mechanism. Figure B-8, Appendix B, shows the components of a 50- and 100-mm-diam flow sensor prior to assembly. The forward-swept design coupled with the absence of magnetic drag provides improved accuracy and repeatability at lower flow rates. The manufacturer states the following specifications: calibration range $0.3 \mathrm{~m} / \mathrm{sec}$ to $9.1 \mathrm{~m} / \mathrm{sec}$; accuracy \pm 1 percent of full scale; repeatability \pm 0.5 percent; and linearity \pm 0.5 percent. The frequency of the output signal, a low impedance 8-volt d-c square wave, is proportional to the magnitude of the flow rate through the pipe and is transformed into a digital signal and transmitted to a personal computer using RS-485 protocol. During operation of the facility, the measured flow rate can be adjusted to match the target flow rate by changing the speed of the vertical turbine pump. For further information on the flow sensors, see Data Industrial (1994). 


\section{Flow channel and intake system}

As shown previously in Figure 1, flow channels are used at the upstream end of the facility to guide water from the discharge pipes to the upstream boundary of the beach. Likewise, on the downstream end of the facility, flow channels guide the longshore current from the downstream boundary of the beach to the vertical turbine pumps. Each of the flow channels is $0.75 \mathrm{~m}$ wide. The two sidewalls of the channels consist of fabricated aluminum flow guides. A continuous neoprene gasket is used to seal the interface between the flow guides and the reinforced concrete floor and sidewalls of the facility, so that each flow channel is independent and water tight. To ensure maximum flexibility of the facility, the recirculation system has been designed with the capability to conduct experiments with water levels ranging from 0.5 to $1.0 \mathrm{~m}$. As a result, 20 sumps were constructed to ensure vortex free operation of the vertical turbine pumps at maximum discharge, and hence maximum drawdown, with a minimum operating water level of $0.5 \mathrm{~m}$. Each sump is constructed of reinforced concrete and is 0.75 $\mathrm{m}$ wide, $1.5 \mathrm{~m}$ long, and $1.2 \mathrm{~m}$ deep (Figure B-6, Appendix B). Hence, design of the intake system to each pump consists of a straight open flow channel with a sump at the downstream end of the channel. Turns and obstructions in the intake system were minimized to avoid the possibility of eddy currents causing submerged vortices under high flow conditions.

\section{Hydraulic Characteristic Curves}

By the use of variable speed motors, the discharge of each pump can be varied to suit the recirculation requirements for a given test condition. Figure 18 shows the performance curve for Pump No. 10 operating at speeds of 540, 1,080, 1,440 , and 1,800 rpm, which correspond to $30,60,80$ and 100 percent of the nominal operating speed of $1,800 \mathrm{rpm}$. The manufacturer supplied the performance curve for a pump speed of $1,800 \mathrm{rpm}$. The performance curves representing slower pump speeds were calculated using the standard homologous pump equations.

Figure 18 also shows two characteristic curves for Pump No. 10 piping system. These two curves represent head loss through the 70-m-long, 200-mmdiam main pipeline combined with the head loss through the 5-m-long flow measurement section of 100- and 50-mm-diam (FS = Flow Sensor in legend of figure). The intersections of the pump performance curves with the two characteristic pipe curves define the range of operating conditions of the combined pump-and-pipe system. The following technique is used to meet the design requirement of a pumping range between 10 and 100 percent of the maximum discharge of each pump. Point No. 1 in the figure, shows that the maximum discharge through System No. 10 is approximately $75 \mathrm{\ell} / \mathrm{sec}$. The pump speed then can be reduced to 30 percent $(540 \mathrm{rpm})$ of the nominal pump speed of $1,800 \mathrm{rpm}$ depicted by Point No. 2. However, if the pump speed is reduced to less than approximately 30 percent of the nominal speed, the pump discharge will plummet to zero because of the inadequate centrifugal and axial forces at the impeller. Therefore, to obtain lower discharge rates, the valve in the larger pipe is closed and the flow rate is measured using the flow sensor in the smaller pipe. However, this increases the head loss in the piping system. To overcome this additional head loss, the pump speed is increased to approximately $1,680 \mathrm{rpm}$ 
(Point No. 3) on the second characteristic pipe curve. To obtain lower flow conditions, the pump discharge can again be reduced until Point No. 4 is reached, corresponding to 30 percent of the nominal speed. At Point No. 4, the discharge through the piping system is approximately $7.5 \mathrm{l} / \mathrm{sec}$, which is 10 percent of the maximum discharge of $75 \mathrm{l} / \mathrm{sec}$, at Point No. 1. The 20 pump-and-piping systems were designed to have this same functional capability.

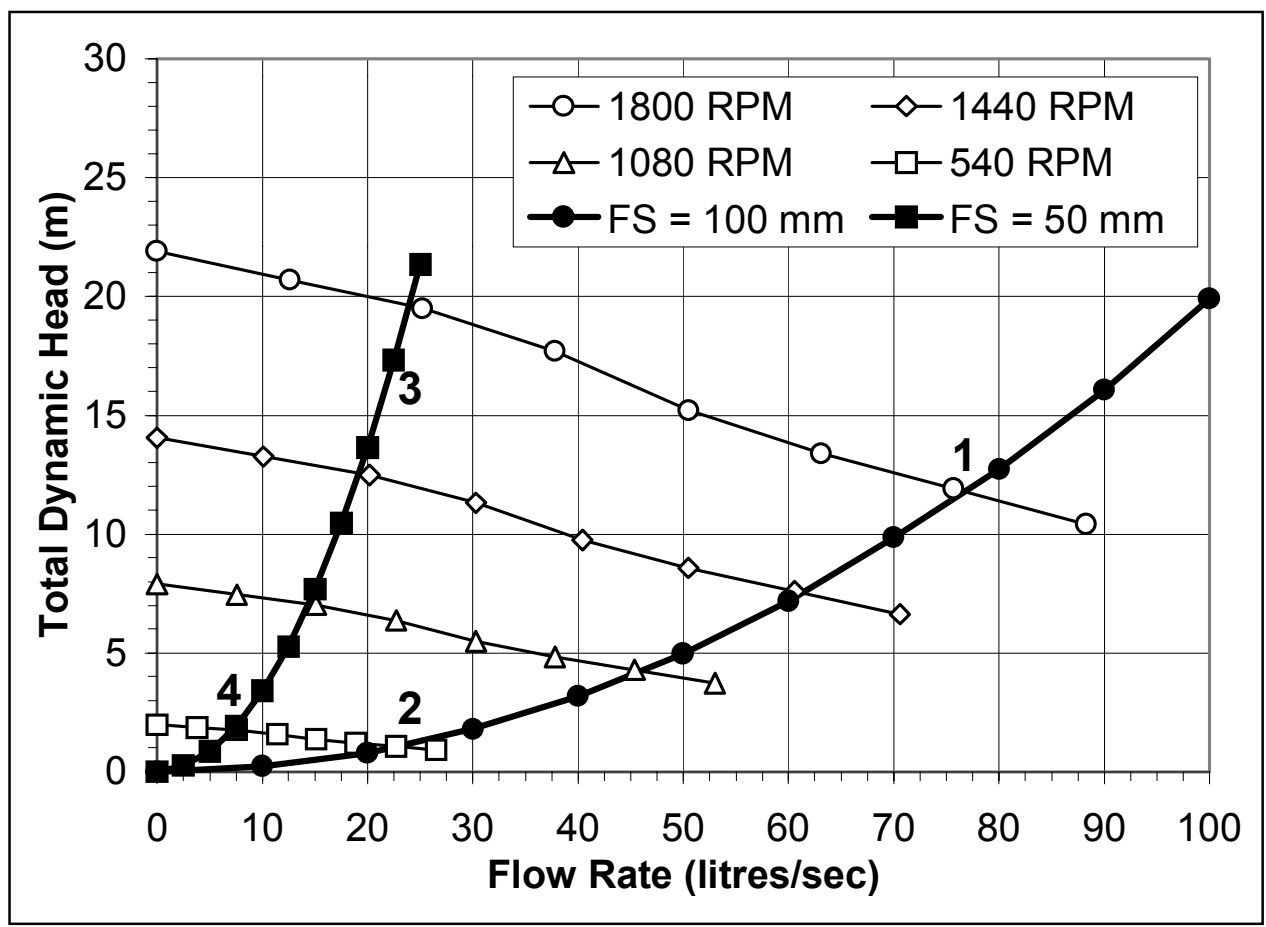

Figure 18. System No. 10: Pump performance and characteristic pipe curves

\section{Calibration and Testing}

\section{Relationship between pump discharge and frequency}

After the recirculation system was constructed, tests were conducted to develop a relationship between pump discharge rates and frequency of the motor controllers for each of the 20 pump-and-piping systems. The discharge from each system was measured using the factory calibration equations supplied with each of the 40 in-line flow sensors. Tests were conducted starting with low-flow conditions by closing the valve in the larger pipeline and allowing the flow to pass through the smaller pipeline. Each system was evaluated for the full range of frequencies of the motor controllers starting at $15 \mathrm{~Hz}$ and increasing up to 60 $\mathrm{Hz}$ in increments of $5 \mathrm{~Hz}$, for a total of 10 data points per calibration curve. Each data point represents the average flow rate measured for 500 -sec duration and sampled at $20 \mathrm{~Hz}$. This series of 10 tests was repeated for the high-flow conditions by closing the valve in the smaller pipeline and allowing the flow to pass through the larger pipeline. 
Figure 19 shows the results for pump-and-piping System No. 10. The lower curve represents the relationship between pump discharge and frequency of the motor controller when water is flowing past the flow meter in the smaller pipeline (50-mm diam). The upper curve represents the second series of tests with water flowing past the flow meter in the larger pipeline (100-mm diam). The two best-fit lines passing through the two sets of data represent the results from regression analysis. The relationship between pump discharge and frequency was very linear, as predicted by theory. This was true for the 20 systems. As listed previously in Table 1, several of the systems have identical pumps, motors, and variable frequency motor controllers. However, because the length of each pipeline varies for each system, the discharge rates at a given frequency for each of these systems are not identical. Therefore, there is a unique set of calibration curves for each of the 20 systems.

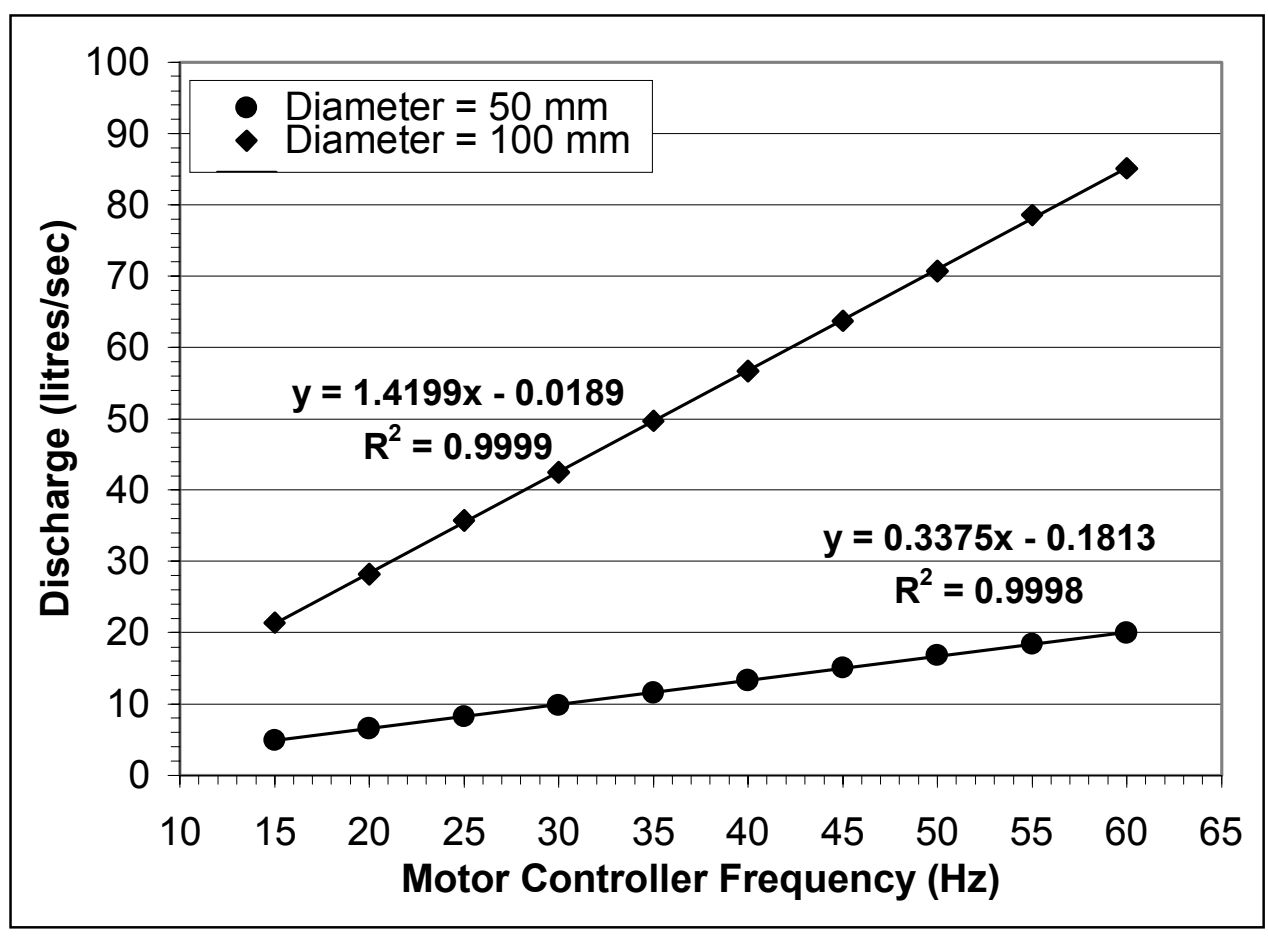

Figure 19. System No. 10: Relationship between pump discharge and frequency of the motor controller

Using these calibration curves, the operator of the facility can readily determine the frequency settings for each of the pumps, once the desired discharge rate for each system has been determined. Two years after conducting these calibration tests, at the time of writing this report, experience has shown that these calibration curves have an accuracy of within +2 to 3 percent. Therefore, these calibration curves work very well as a first approximation of the frequency settings of the motor controllers. The facility operator then would adjust the frequency settings to bring the measured discharge rates to within \pm 1 percent of the desired values. After the low-flow or the high-flow mechanisms have been selected for each pump, the process of converging in on the correct frequency for the motor controllers requires approximately $30 \mathrm{~min}$. 


\section{Pumping steadiness and measurement repeatability}

As mentioned previously, another system requirement for the recirculation system was the capability of maintaining constant discharge rates for several hours of continuous operation. This was needed to accommodate the time scales associated with conducting sediment transport experiments. To help meet this requirement, all of the components procured for the recirculation system were of high quality design and construction. Nonetheless, maintaining constant discharge rates for several hours of operation was still a concern. Therefore, tests were conducted to verify the steadiness of flow through each of the 20 systems and to evaluate the repeatability of the in-line flow sensors.

Tests were conducted by operating all of the pumps for $8 \mathrm{hr}$ of continuous operation to represent the case where an experiment was run continuously all day long; an extreme case. The in-line flow sensors were sampled at $20 \mathrm{~Hz}$ and the average flow rate was recorded every $30 \mathrm{sec}$ in the 20 systems. Therefore, each data point in the recorded time series represented the average value of the previous $600(20 \mathrm{~Hz} \times 30 \mathrm{sec})$ instantaneous values for each of the 20 systems. For pump-and-piping System No. 10, the standard deviation was $0.40 \mathrm{l} / \mathrm{sec}$ for an average discharge rate of $70 \mathrm{l} / \mathrm{sec}$. Therefore, the average discharge rate remained within +2 percent of the desired flow rate of $70 \mathrm{\ell} / \mathrm{sec}$ during $8 \mathrm{hr}$ of continuous operation. The 20 pump-and-piping systems gave similar results. Unfortunately, it was not possible to determine if this variation was caused by actual oscillations in the pump discharge rate or by errors associated with measuring the flow rate with the in-line flow sensors. Nonetheless, this magnitude of variation is relatively small, and it was concluded that the recirculation system met the design requirements for the facility.

\section{Summary}

This chapter documents the design process used to develop the longshore current recirculation system for the LSTF. Prior to construction, a design wave condition, of $\mathrm{H}_{\mathrm{s}}=0.3 \mathrm{~m}, \mathrm{~T}_{\mathrm{p}}=2.5 \mathrm{~s}$, and $\theta=20 \mathrm{deg}$, with an offshore water depth of $0.9 \mathrm{~m}$, was used to numerically estimate the magnitude and cross-shore distribution of the longshore current that would be generated in the new facility. It was concluded that 20 independent pump-and-piping systems would be required to provide adequate cross-shore resolution to recirculate a broad range of longshore current distributions within the facility.

The 20 independent pump-and-piping systems have a total discharge capacity of $1,250 \mathrm{\ell} / \mathrm{sec}$. Using a combination of variable speed vertical turbine pumps along with a low- and high-flow measurement mechanism in each piping system, the recirculation system has the capability to accurately recirculate between 10 and 100 percent of the maximum design capacity at the 20 cross-shore locations. This unique design allows a wide range of longshore current magnitudes and cross-shore distributions to be accurately controlled and recirculated for a wide range of incident wave conditions, water levels, and bathymetric conditions. 
Calibration and verification of the longshore current recirculation system, as well as 2 years of experience operating the system during fixed-bed and moveable-bed experiments, has demonstrated that the recirculation system meets the design requirements originally developed for the LSTF. 


\section{Sediment Trapping and Dredging Systems}

\section{Introduction}

This chapter describes the design, testing, and verification of the sediment trapping and dredging systems developed for the LSTF. This system is used to measure the magnitude and cross-shore distribution of sand transported along the beach while waves and currents are generated. This system is comprised of the following components:

a. Twenty gravity-feed rectangular sediment traps and the associated support frames.

b. Sixty individual load cells (three for each trap) to obtain real-time measurements of the quantity of sand that accumulates in each trap.

c. A data acquisition system for data collection and real-time display of the quantity of sand simultaneously measured in each trap.

d. A system for dredging sand from the traps and transporting it as a hydraulic slurry through a closed conduit back to the updrift end of the facility.

This chapter begins by describing the design of the major components of the sediment trapping system. The design and general operation of the dredging system is then discussed. The next section describes results obtained from a series of static performance tests conducted with the facility drained and the traps empty, to verify the operation of the 20 sediment traps. The last section discusses results from a set of dynamic performance tests conducted using regular and irregular waves, to assess the structural integrity of the trap design as well as the signal to noise ratio of the measurements. The data acquisition system used to measure the quantity of sand in each trap, along with the real-time display system used to visualize the measured data, will be discussed in Chapter 6. 


\section{Design of Sediment Trapping System}

\section{Sediment traps}

The sediment traps are located at the downdrift end of the facility, and are positioned within the flow channels, as illustrated in Figure 20. A sediment trap is located in each flow channel starting with Channel No. 1 (the most shoreward flow channel) and extending offshore to flow Channel No. 17. Additionally, a sediment trap is located in flow Channel No. 19. The decision to eliminate traps in flow Channels No. 18 and 20 was determined based on cost considerations and the expected low rates of longshore sediment transport in the offshore region. Based on the fall velocity of the sediment used to construct the moveable-bed beach, and the magnitude of the longshore current in the flow channels, the decision was made to maximize the length of these sediment traps. Each trap is approximately $6.0 \mathrm{~m}$ long and $0.6 \mathrm{~m}$ wide. In addition, two shorter traps are located landward of flow Channel No. 1 with the objective of quantifying the longshore sediment transport rate near the still-water shoreline and in the uprush zone. Each "uprush-zone" trap is approximately $2.5 \mathrm{~m}$ long and $0.5 \mathrm{~m}$ wide. These two traps were added to the design after qualitatively observing the magnitude of longshore sediment transport in the swash zone as well as the morphologic changes that occurred in this region during some preliminary moveable-bed experiments.

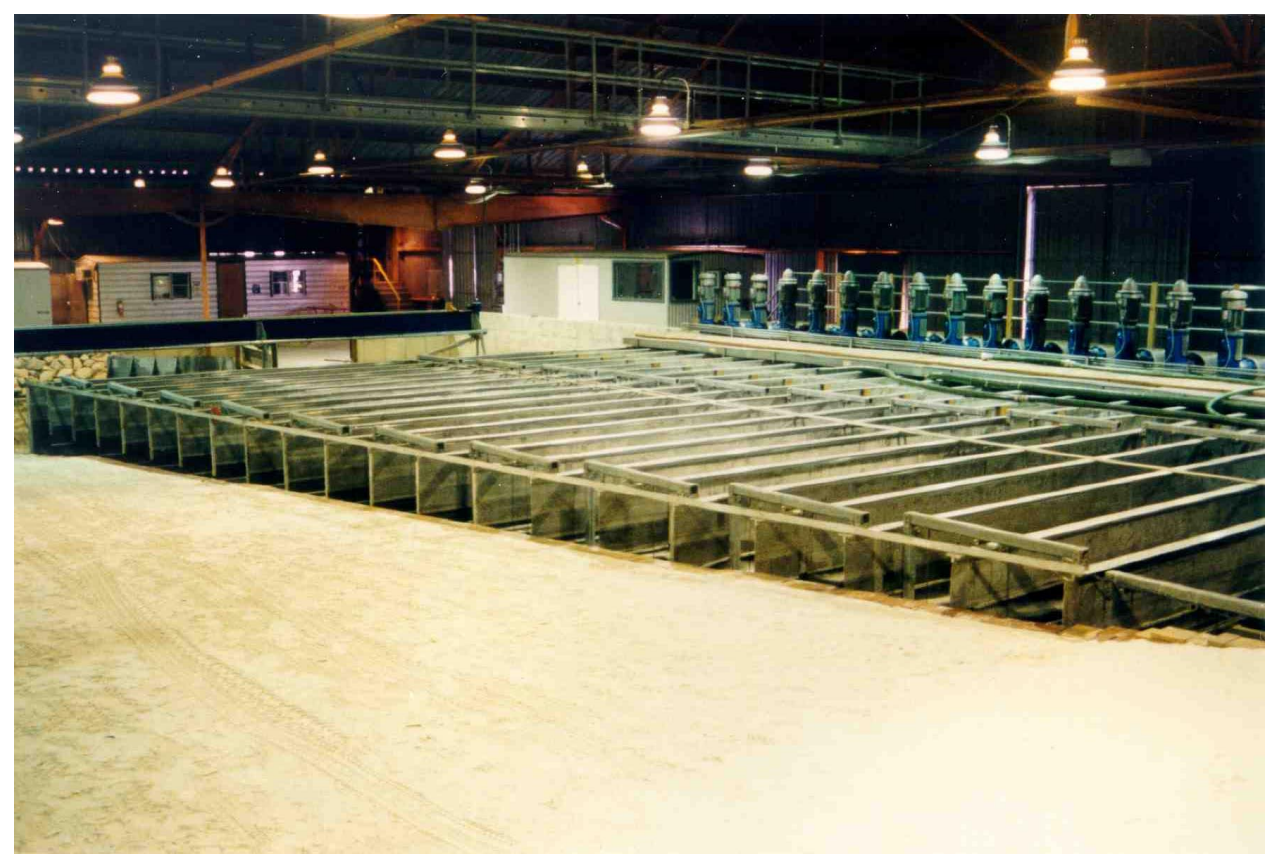

Figure 20. Twenty sediment traps are located inside downdrift flow channels

The dimensions and capacity of each of the traps are tabulated in Table 2, based on the as-built inside dimensions of each trap and assuming that the traps can be completely filled with sand along the entire length of the trap (a very extreme case). The last column lists the capacity of each trap in terms of the 
maximum submerged weight of sand that each trap can hold. In this report, the term "submerged weight" will be used rather than "mass," because this is the quantity the traps measure. These calculations assume that the specific gravity of quartz sand is 2.65 , and that the in situ bulk porosity of the sand in the trap is 0.4 . Therefore, the buoyant unit weight of the sand in the traps is $1,122-\mathrm{kg} / \mathrm{m}^{3}$. As shown in Figure 20, the height of the traps decreases in the offshore direction. This is dictated by the fact that the elevation of the beach decreases in the offshore direction. One of the design criteria was to select the height of each trap so that the brim elevation of the trap is slightly lower than the elevation of the concrete beach. Therefore, in an extreme event, if a portion of the moveable bed eroded close to the elevation of the concrete, sediment could be transported into the traps and not be obstructed. This decision also makes it possible to remove the entire sand beach in the future to conduct additional fixed-bed experiments without the traps obstructing the longshore current. In addition, to simplify construction and assembly, the trap height decreases in sets of two or three traps. Therefore, there are only 10 different sizes. This also allows mechanical components on adjacent traps to be temporarily interchanged, if in need of repair.

\begin{tabular}{|c|c|c|c|c|c|c|}
\hline \multicolumn{7}{|c|}{$\begin{array}{l}\text { Table } 2 \\
\text { Dimensions and Capacities of the } 20 \text { Sediment Traps }\end{array}$} \\
\hline Trap No. & $\begin{array}{l}\text { X-Loc. } \\
\text { m }\end{array}$ & $\begin{array}{l}\text { Inside } \\
\text { Height } \\
\text { m }\end{array}$ & $\begin{array}{l}\text { Inside } \\
\text { Length } \\
\text { m }\end{array}$ & $\begin{array}{l}\text { Inside } \\
\text { Width } \\
\text { m }\end{array}$ & $\begin{array}{l}\text { Volumetric } \\
\text { Capacity } \\
\mathrm{m}^{3}\end{array}$ & $\begin{array}{l}\text { Maximum } \\
\text { Submerged } \\
\text { Weight of } \\
\text { Sand } \\
\text { kg }\end{array}$ \\
\hline $\begin{array}{l}-2 \\
\end{array}$ & 1.875 & 0.55 & 2.44 & 0.50 & 0.67 & 749 \\
\hline-1 & 2.625 & 0.55 & 2.44 & 0.50 & 0.67 & 749 \\
\hline 1 & 3.375 & 0.50 & 6.10 & 0.56 & 1.71 & 1917 \\
\hline 2 & 4.125 & 0.50 & 6.10 & 0.56 & 1.71 & 1917 \\
\hline 3 & 4.875 & 0.46 & 6.10 & 0.56 & 1.58 & 1772 \\
\hline 4 & 5.625 & 0.46 & 6.10 & 0.56 & 1.58 & 1772 \\
\hline 5 & 6.375 & 0.37 & 6.10 & 0.56 & 1.28 & 1432 \\
\hline 6 & 7.125 & 0.37 & 6.10 & 0.56 & 1.28 & 1432 \\
\hline 7 & 7.875 & 0.37 & 6.10 & 0.56 & 1.28 & 1432 \\
\hline 8 & 8.625 & 0.32 & 6.10 & 0.56 & 1.10 & 1238 \\
\hline 9 & 9.375 & 0.32 & 6.10 & 0.56 & 1.10 & 1238 \\
\hline 10 & 10.125 & 0.32 & 6.10 & 0.56 & 1.10 & 1238 \\
\hline 11 & 10.875 & 0.27 & 6.10 & 0.56 & 0.93 & 1044 \\
\hline 12 & 11.625 & 0.27 & 6.10 & 0.56 & 0.93 & 1044 \\
\hline 13 & 12.375 & 0.25 & 6.10 & 0.56 & 0.84 & 947 \\
\hline 14 & 13.125 & 0.20 & 6.10 & 0.56 & 0.67 & 752 \\
\hline 15 & 13.875 & 0.20 & 6.10 & 0.56 & 0.67 & 752 \\
\hline 16 & 14.625 & 0.15 & 6.10 & 0.56 & 0.52 & 582 \\
\hline 17 & 15.375 & 0.15 & 6.10 & 0.56 & 0.52 & 582 \\
\hline 18 & 16.125 & No Trap & & & & \\
\hline 19 & 16.875 & 0.11 & 6.10 & 0.56 & 0.39 & 437 \\
\hline 20 & 17.625 & No Trap & & & & \\
\hline
\end{tabular}

\section{Trap support frames and degrees of freedom}

A pair of traps is supported by two mounting frames, which are made of rectangular galvanized steel tubing. Each frame spans two flow channels and supports two traps, one frame at the upstream end of the trap and one at the downstream end, as shown in Figure 21. The support frames are bolted to the concrete floor of the wave basin. Each trap is suspended at two points near the upstream corners of the trap, where most of the sand accumulates, and one 
suspension point in the center of the downstream end of the trap. At each suspension point a load cell is attached to the frame, and the trap is hung from the load cell with a stainless steel cable. A stainless steel threaded rod at the upper end of each cable provides a mechanism for leveling the traps during installation. Three suspension points were used, rather than four (one on each corner) so that the load cells only restrain the traps from vertical motion, and not in the other 5 degrees of freedom. This guarantees that the vertical force on the load cell is not influenced by, for example, the vertical component of a lateral load induced by hydrodynamic forces, or from a slight rotation of the trap induced by a nonuniform distribution of sand in the trap. This type of design provides the most accurate measurement of the quantity of sand in each trap, and also makes it possible to calculate the center of mass of the mound of sand that accumulates in each trap. Although calculating the center of mass is not a priority, it could be useful for determining such things as whether or not there is a relationship between the longshore position of the center of mass in a given trap, and the speed of the longshore current in that flow channel. A prototype sediment trap was constructed and tested during the design phase to optimize the design prior to construction of the 20 traps. Several improvements to the design were made while evaluating the prototype.

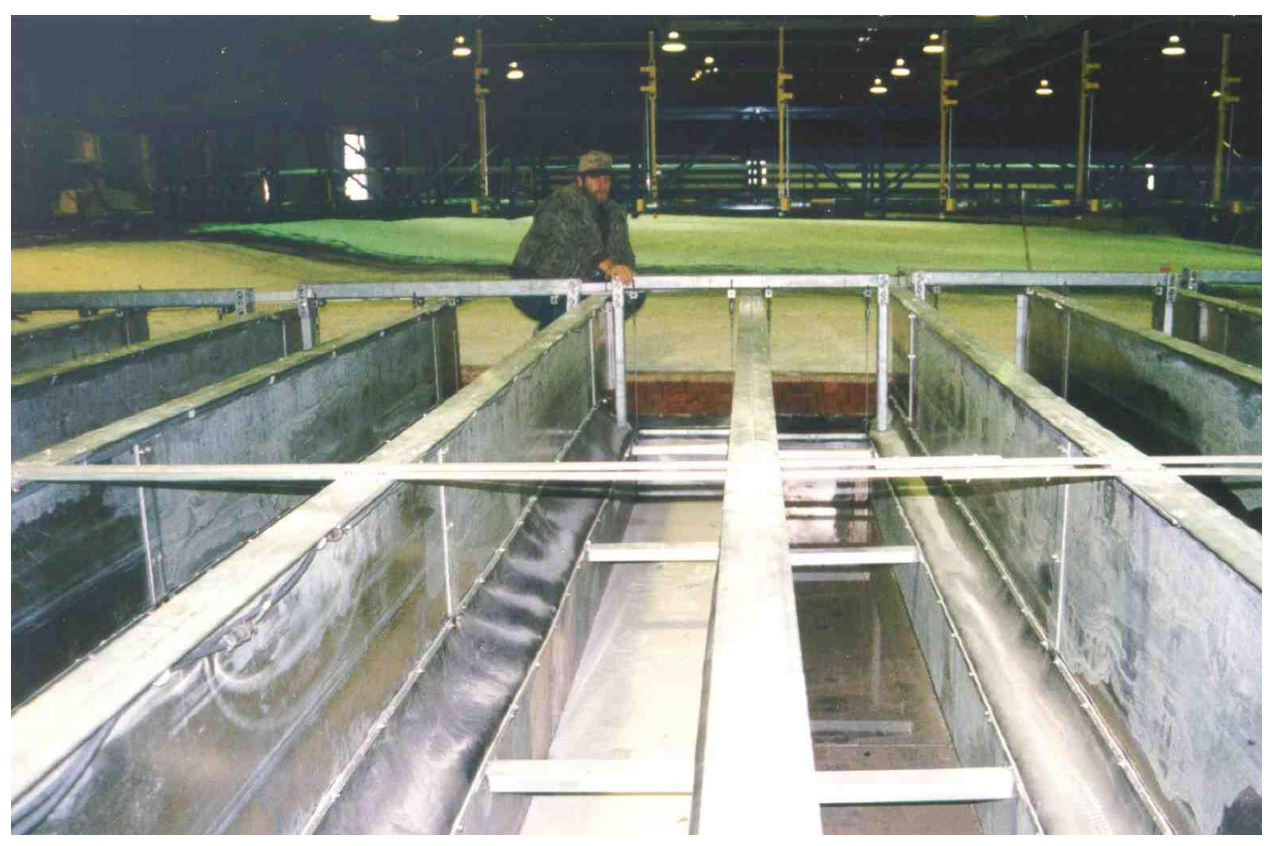

Figure 21. Two sediment traps are suspended from a set of support frames via stainless steel cables and load cells

\section{Load cells and design capacities}

Hermetically sealed load cells (shear beams) are used to measure the quantity of sand in each trap. The load cells are made of stainless steel construction and are airtight and splash proof. The load cells are bolted to the bottom surface of the upper horizontal member of the support frame so that the entire sediment trapping system has a low profile, as shown in Figure 22. This provides 
clearance for the instrumentation bridge to traverse over the top of the sediment traps during calibration of the wave gauges and the profile indicator. This design also keeps all of the mechanical components below the upper surface of the support frames thereby eliminating interference with the suction hose of the slurry pump during the dredging operation.

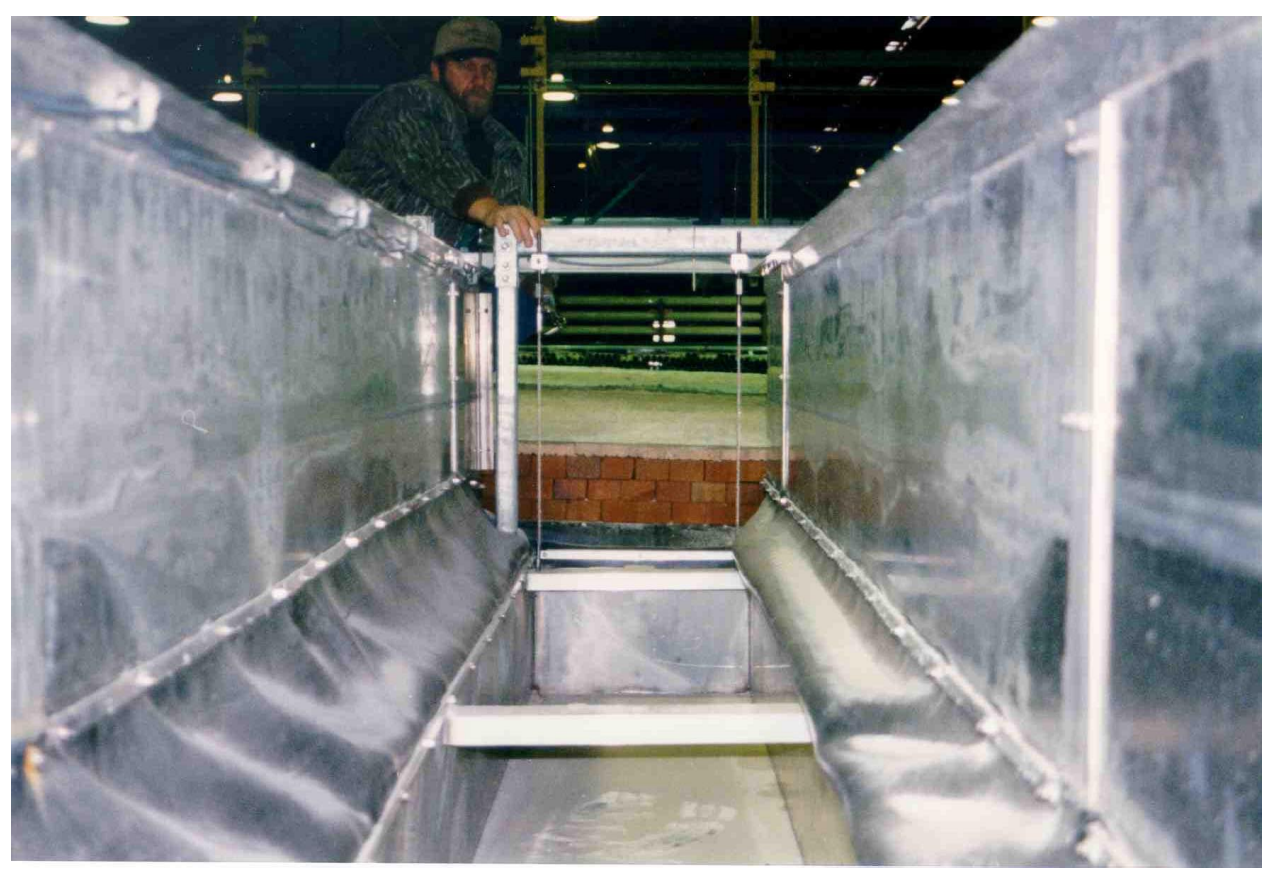

Figure 22. Stainless steel, splash-proof load cells are bolted to the bottom of the support frames to support the sediment traps. A neoprene rubber seal is used between the flow channel walls and the sediment traps

Three different size load cells were used with rated capacities of 250-, 500-, and $1,000-\mathrm{kg}$, depending on the design requirements for each trap. The load cells can temporarily support 150 percent of the manufacturers rated capacities without experiencing permanent damage. The manufacturer specifies an accuracy of \pm 0.05 percent of the rated capacities, which includes errors resulting from linearity, repeatability, and hysteresis. During the testing phase of the prototype sediment trap, it was concluded that the load cells are very robust with respect to fluctuations in humidity. However, the load cells are more sensitive to fluctuations in temperature. Errors induced by temperature fluctuations are minimized by performing a "shunt-cal" on all 60 load cells, prior to each experiment, and this procedure will be discussed in Chapter 8.

Figure 23 graphically illustrates the design capacity of each of the sediment traps. Two series of data are shown in the figure. The first series represents the maximum capacity of each trap based on the manufacturers rated capacities of the three load cells supporting each trap. The second series represents the maximum submerged weight of sand that each trap can hold, based on the volumetric capacity of each trap. These values are identical to the values listed in the last column of Table 2. As discussed previously, this assumes that the traps are completely submerged and completely filled with sand along the entire 
length of the trap (a very extreme case). As illustrated in the figure, the maximum capacity of each trap is limited by the maximum submerged weight of sand that the trap can hold, based on the volume of the trap, rather than the rated capacities of the load cells. Selection of the size of load cells used on each trap was made to maximize the accuracy of the measurements, while at the same time protecting the load cells from the possibility of over loading.

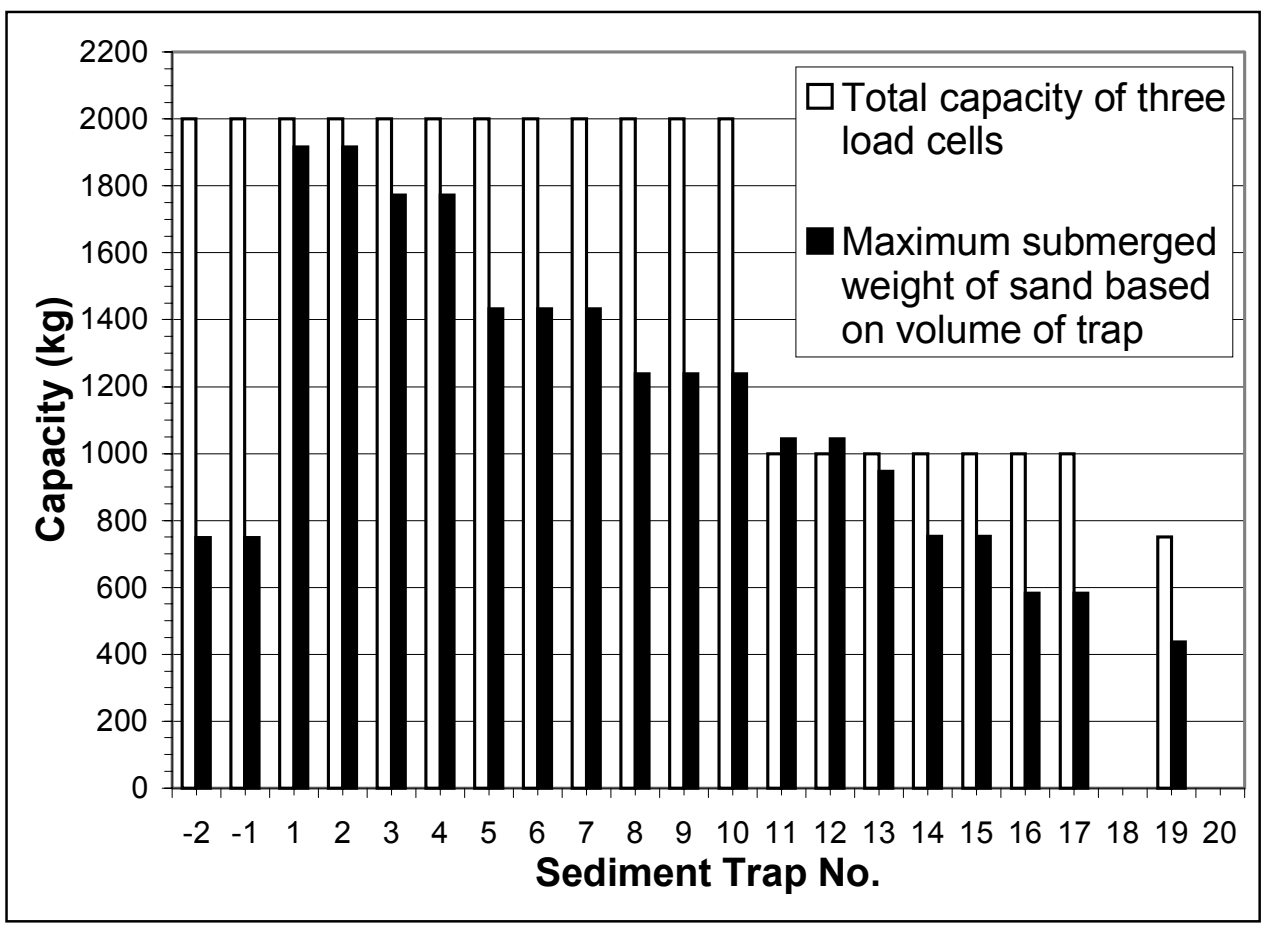

Figure 23. Design capacity of the sediment traps in terms of the total load cell capacity and the maximum submerged weight of sand that each trap can hold based on the volumetric capacity of each trap

\section{Sealing the perimeter of the traps}

To prevent sand from filling in the gap beneath a trap, each trap is sealed on the upstream edge and on the two side walls using neoprene rubber sheeting, as shown previously in Figure 22. The seal between a flow channel side wall and the inside wall of the trap is made by compressing the rubber sheet between the wall and an aluminum clamping bar and then bolting the clamping bar to the wall. The sheet is clamped on both the trap and flow channel walls. At the upstream end of the traps, a single rubber sheet was laid along the entire width of the concrete beach to form the upstream seal for all the traps. The sheet was slit at each flow channel wall and slid into place. Within each trap, this rubber sheet was clamped to the upstream edge of the trap in the same manner that the sidewalls were sealed. The other end of the sheet was compressed between the concrete beach and the stack of bricks used to build the adjustable boundary at the downstream end of the beach. The sand beach was back-filled against the bricks to cover any exposed rubber sheeting which extended beyond the upstream end of the brick boundary. A sand-tight seal was created between the 
upstream and side wall sheets by trimming the overlapping upstream sheet and binding the two sheets with a special cement and stainless steel rivets. Silicone caulking also was used to aid in the sealing process.

\section{Dredging System}

During the course of an experiment that has been in operation for a significant amount of time, the sand that has accumulated in the traps is dredged from the traps and discharged onto the updrift end of the beach. Depending on the magnitude of the waves and currents in a particular experiment, the quantity of sand that accumulates in the traps prior to dredging can range from 1,000 to $2,000 \mathrm{~kg}$, or even greater. The decision to dredge the traps is dictated by one or both of the following criteria:

a. The updrift end of the beach has eroded significantly, causing a perturbation in the bathymetry and, hence, the nearshore processes in that region, because sand is not fed onto the beach during the experiment.

b. Sand has accumulated at the updrift end of the sediment traps, causing a potential obstruction to the flow of current into the downdrift flow channels.

An 11-hp trash pump is used to dredge and transport a sand slurry through a series of reinforced flexible hoses to the upstream end of the beach. It is common practice in the construction industry to always select the diameter of the suction hose to be larger than the diameter of the discharge hose and to minimize the length of the suction hose to avoid the possibility of cavitation inside the pump chamber. For this pump application, however, it was not possible to follow this rule of thumb. The standard size suction hose to fit this pump has a diameter of $100 \mathrm{~mm}$. However, it proved to be far too cumbersome for one person to manually move this large-size hose from trap to trap when filled with a sand slurry during the dredging operation. Therefore, after some trial and error testing, the optimum solution was to use a $50-\mathrm{mm}$ suction hose and a $100-\mathrm{mm}$ discharge hose. All of the hose is designed with wire braided noncollapsible walls so that the hose does not collapse when draped over the top of the walls of the flow channels and exterior walls of the basin.

The sand slurry is discharged directly onto the upstream end of the beach. A diffuser pipe approximately $3.0 \mathrm{~m}$ in length is used to distribute the sand slurry onto the beach to minimize erosion of the beach. The diffuser pipe can be moved to different locations across the width of the surf zone so that the sand slurry fills regions where the greatest amount of erosion has occurred. This system has proved to work quite effectively.

\section{Static Performance Tests}

\section{Tests with traps empty and the facility drained}

After the traps had been fully constructed and sealed with neoprene rubber, several tests were conducted to assess the mechanical performance and 
signal-to-noise ratio (SNR) of the 20 sediment traps. The first test involved measuring the tare-weight of the traps, as the empty traps hung freely in the air and the facility was drained. Data were collected on all 60 load cells simultaneously for a duration of $80 \mathrm{sec}$, at a sampling rate of $1 \mathrm{~Hz}$, which would be the typical sampling frequency used during experiments. The average, standard deviation, maximum, and minimum values of the instantaneous weights in each time series were calculated. The total tare-weight of all 20 traps was calculated to be $2,888 \mathrm{~kg}$. This is essentially the total weight of the materials (aluminum and stainless steel) used to fabricate the traps.

Figure 24 shows the minimum and maximum value divided by the average value for each of the 60 time series. The greatest ratio of either the minimum or maximum value divided by the average value was approximately four percent, and only one percent on average (for all 60 load cells). The third time series is plotted as one plus the ratio of the standard deviation divided by the average value. This ratio was at most 0.6 percent and about 0.3 percent on average.

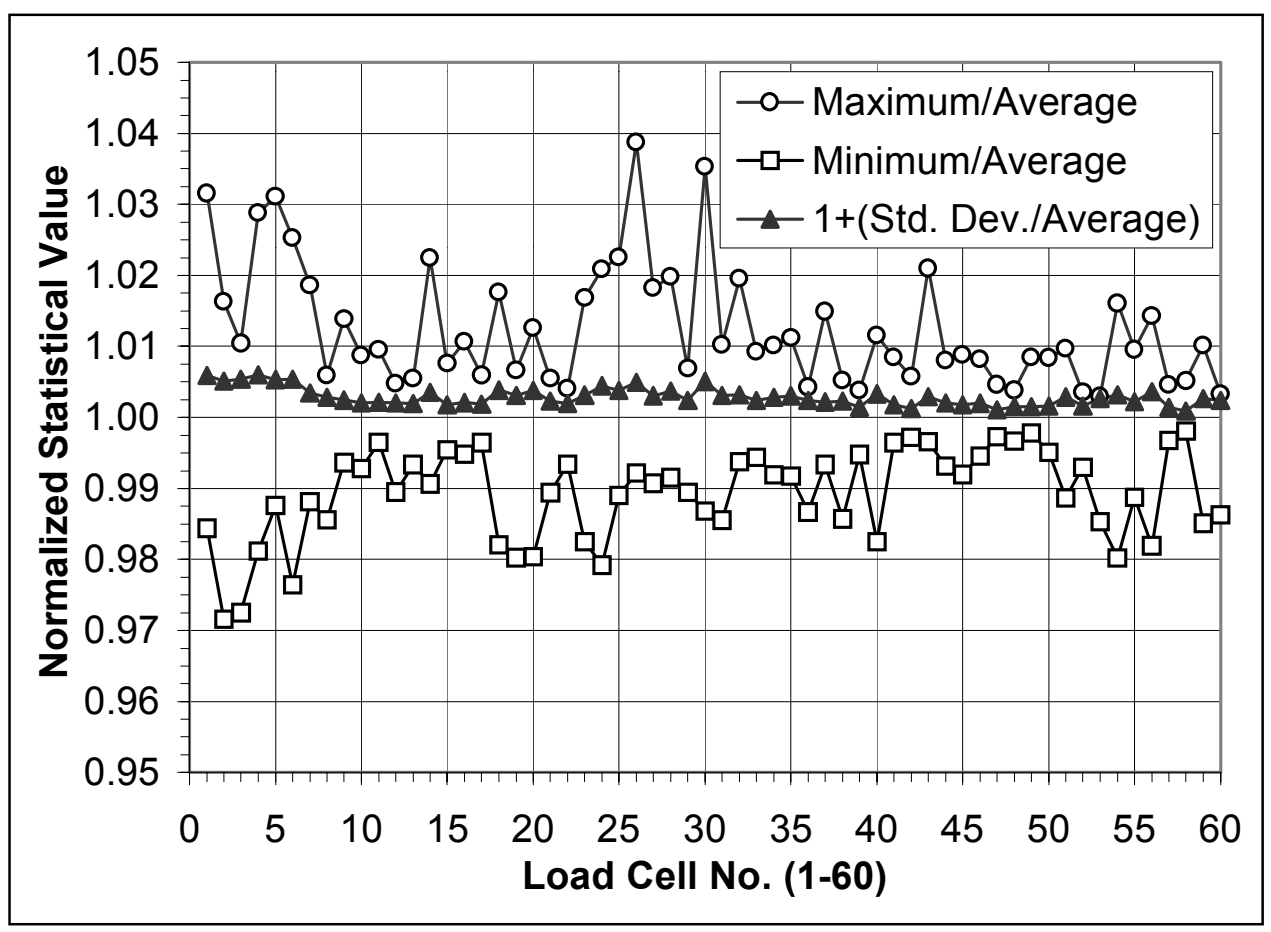

Figure 24. Statistical parameters normalized by the average value in the time series, obtained from each load cell during tare-weight tests conducted with the traps empty and the facility drained

Figure 25 shows the standard deviation of each time series. These are the same data as the third time series of Figure 24 but plotted in terms of absolute weight, rather than normalized by the average value. As shown, the standard deviation was always less than $0.35 \mathrm{~kg}$ for average load cell readings ranging from 22 to $97 \mathrm{~kg}$. On average, the standard deviation was about $0.15 \mathrm{~kg}$. This magnitude of the SNR for the 60 load cells is considered excellent. 


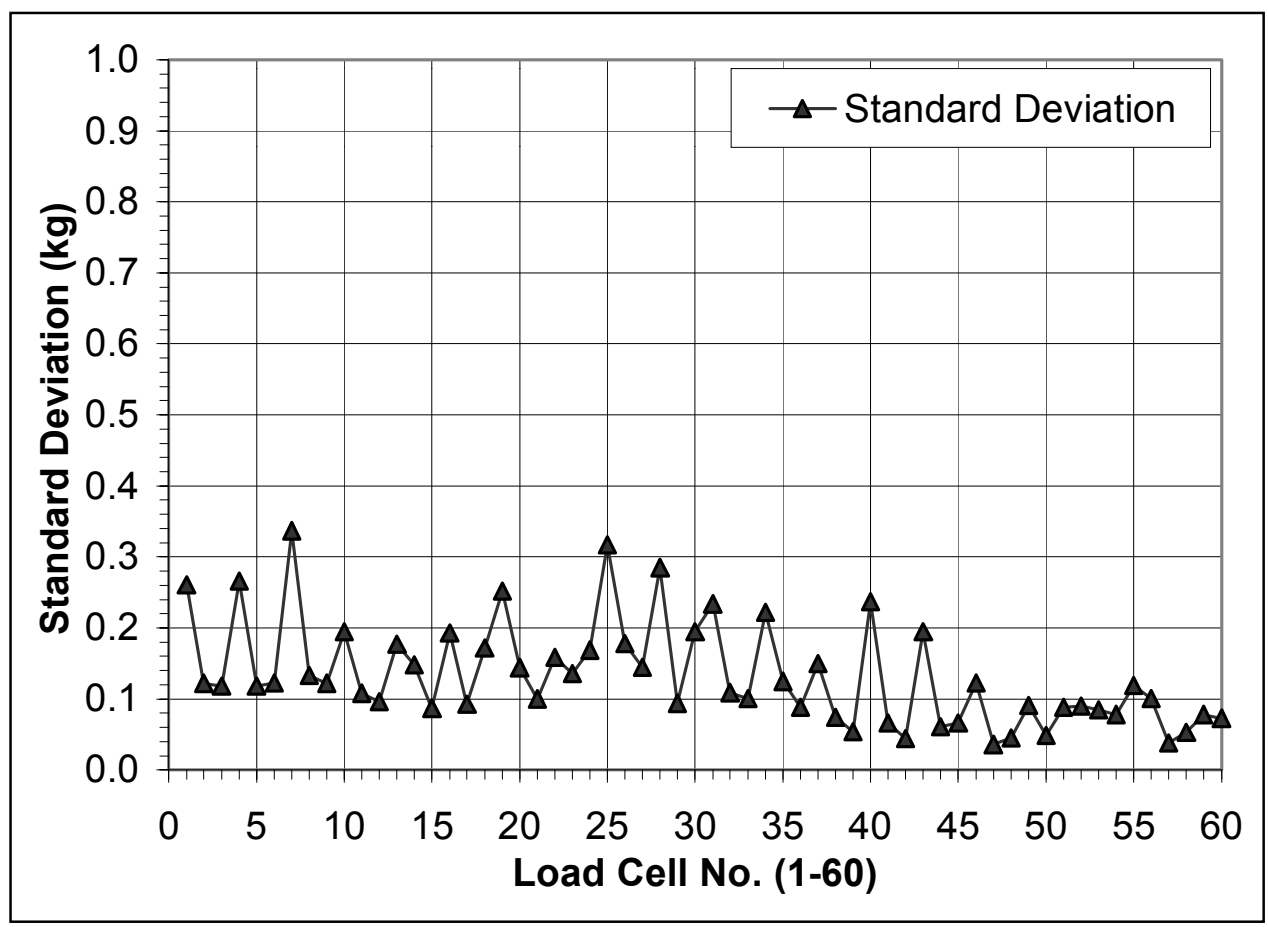

Figure 25. Standard deviation of load cell readings measured during tare-weight tests conducted with the traps empty and the facility drained

Two other tests were performed with the facility completely drained to assess the accuracy of the weight recorded by each trap. The first test involved placing a known weight of approximately $120 \mathrm{~kg}$ at the upstream end of the trap. The total weight in the trap was measured and compared to the total weight measured when the weight was placed at the center, and finally the downstream end of the trap. This process was repeated for the 20 traps. This test was conducted to assess the effect of various distributions of sand along the length of the trap. Results showed that the maximum variation in the sum of the three load cells, for all three positions, did not exceed $0.5 \mathrm{~kg}$, a maximum error of less than 0.5 percent.

The final test conducted with the traps empty and the facility drained involved placing the known weight of $120 \mathrm{~kg}$ in the center of Trap No. 1, and then repeating the measurement for each of the 20 traps. The error in the total weight recorded in each trap was less than $1 \mathrm{~kg}$ from the known weight of 120 $\mathrm{kg}$, which is considered to be excellent. The results of these tests showed that the 20 sediment traps are very consistent and accurate with the traps empty and the facility drained.

\section{Removing air bubbles from beneath the rubber seals}

A number of tests were performed with the wave basin filled to a water depth of $0.9 \mathrm{~m}$, the normal operating water level for the moveable-bed experiments. The traps were completely submerged and filled with water. As the facility was being filled, it was observed that air bubbles would rise to the surface, 
presumably from below the neoprene rubber seal along the sides and upstream end of the traps. Shortly after the facility had been filled, the submerged weight of the traps was measured and the total weight of the 20 traps was calculated to be $1,712 \mathrm{~kg}$ (relative to $2,888 \mathrm{~kg}$ measured with the traps empty and the facility drained). It was necessary to remove air from beneath the rubber seals. A rod was used to agitate each trap about the downstream suspension point, which released air pockets. The traps were reweighed, and the total trap weight was calculated. The operator then spent a total of $15 \mathrm{~min}$ jostling the downstream end of each trap, allowing a significant amount of air to escape from beneath the rubber seals. The total weight of the traps was then calculated to be $1,748 \mathrm{~kg}$, an increase of $36 \mathrm{~kg}$. In other words, about $36 \ell$ of air had escaped from below the seals during the jostling process. The pockets of air below the seals had reduced the total effective trap weight by about 2 percent. The weight of some individual traps increased by as much as 6 percent.

If air is not released from below the seals, the air pockets may escape during an experiment, creating a potentially significant error in the quantity of sand measured in each trap. Based on the results from this experiment, it has become a standard procedure to remove as many air pockets as possible from beneath the rubber seals each time the facility is refilled and prior to conducting an experiment.

\section{Tests with the facility full of water}

The last set of static tests was performed to assess the influence of the location of the load in the trap. A weight of $96 \mathrm{~kg}$ was measured at the upstream, center, and finally the downstream end of the trap in a similar manner as was discussed previously with the traps empty and the facility drained. For all three positions, the maximum variation in the sum of the three load cells did not exceed $1.0 \mathrm{~kg}$, a maximum error of approximately 1 percent. These errors are slightly larger than the errors measured with the traps empty and the facility drained.

Static tests then were conducted to quantify the measurement accuracy of all 20 traps, in a similar manner as previously conducted with the traps empty and the facility drained. A weight of $96 \mathrm{~kg}$ was placed in the center of the first trap, measured, and then the test was repeated for all 20 traps. The average measured weight for the 20 traps was $95.81 \mathrm{~kg}$. The maximum and minimum weights recorded were 98.5 and $91.8 \mathrm{~kg}$, respectively. The standard deviation was $1.5 \mathrm{~kg}$, or approximately 1.5 percent of the average. These errors are slightly larger than the errors measured with the facility drained. However, these results are within the design criteria of developing a sediment trapping system with the ability to measure the average submerged weight of sand in the 20 traps with an absolute accuracy of 3 percent.

\section{Dynamic Performance Tests}

Dynamic tests were performed to examine the structural integrity of the trap design while waves and currents were generated in the facility. Three tests were conducted. The first test was conducted by running all 20 pumps in the LSC 
recirculation system at approximately half of the maximum discharge rate. The physical response of the traps was observed, and data were collected and evaluated. The traps remained essentially motionless during forcing with currents alone and did not seem to affect the variability of the measurements. The range of fluctuations was similar to that observed during the static tests with the facility drained.

A second and third test were conducted by including regular and irregular wave forcing to the currents, respectively. The wave and current conditions were the same as those previously used during the fixed-bed hydrodynamic experiments. The downdrift wave guide, constructed of marine plywood, was removed for these two tests to assess the behavior of the traps in a "worst-case" scenario, since the wave guide significantly reduces the amount of wave action within the flow channels. There was no significant movement of the traps during any of these tests, although they did vibrate minimally during the second and third test if waves were generated.

Figure 26 illustrates the range of variability in the measured time series during the second and third test. The standard deviation for each of the 60 load cells is plotted for both the regular and irregular wave cases. Load Cell No.1 is located at the onshore end and load cell No. 60 is located at the offshore end. As anticipated, the standard deviations decreased significantly close to the shoreline because wave heights are much smaller in that region. In general, the two time series appear to be very similar with average values of the standard deviation of approximately $3.0 \mathrm{~kg}$ further offshore. These variations are significantly larger than in the static test cases; however, it is possible to numerically filter the data using a filtering routine during postprocessing of the data (Chapter 8). In addition, these fluctuations were reduced considerably after the downdrift waveguide was reinstalled, which will be the case for the majority of the LST experiments.

Prior to these tests, there was concern that for regular wave forcing, the traps would be more severely agitated because the hydrodynamic loading would occur at a constant frequency. However, this was not the case, as shown in Figure 26. During the regular wave case two load cells experienced a significantly high standard deviation, but this was not attributed to the regular wave forcing, because this increase only occurred on 2 of the 60 load cells.

\section{Summary}

This chapter documents the design, operation, and performance of the sediment trapping and dredging system developed for the LSTF. The mechanical design of the sediment trapping system has proven to be structurally robust. The dredging system has proven to work effectively and can be operated by one person. Results from the static performance tests with the facility both drained and filled with water showed that the 20 traps have an accuracy of well within the design criteria for the system. The dynamic performance tests showed that pumping a longshore current in the absence of wave forcing has an insignificant effect on the variability of the measured load cell data. However, combined wave and current forcing does increase the variability of the output signal from the load cells. 


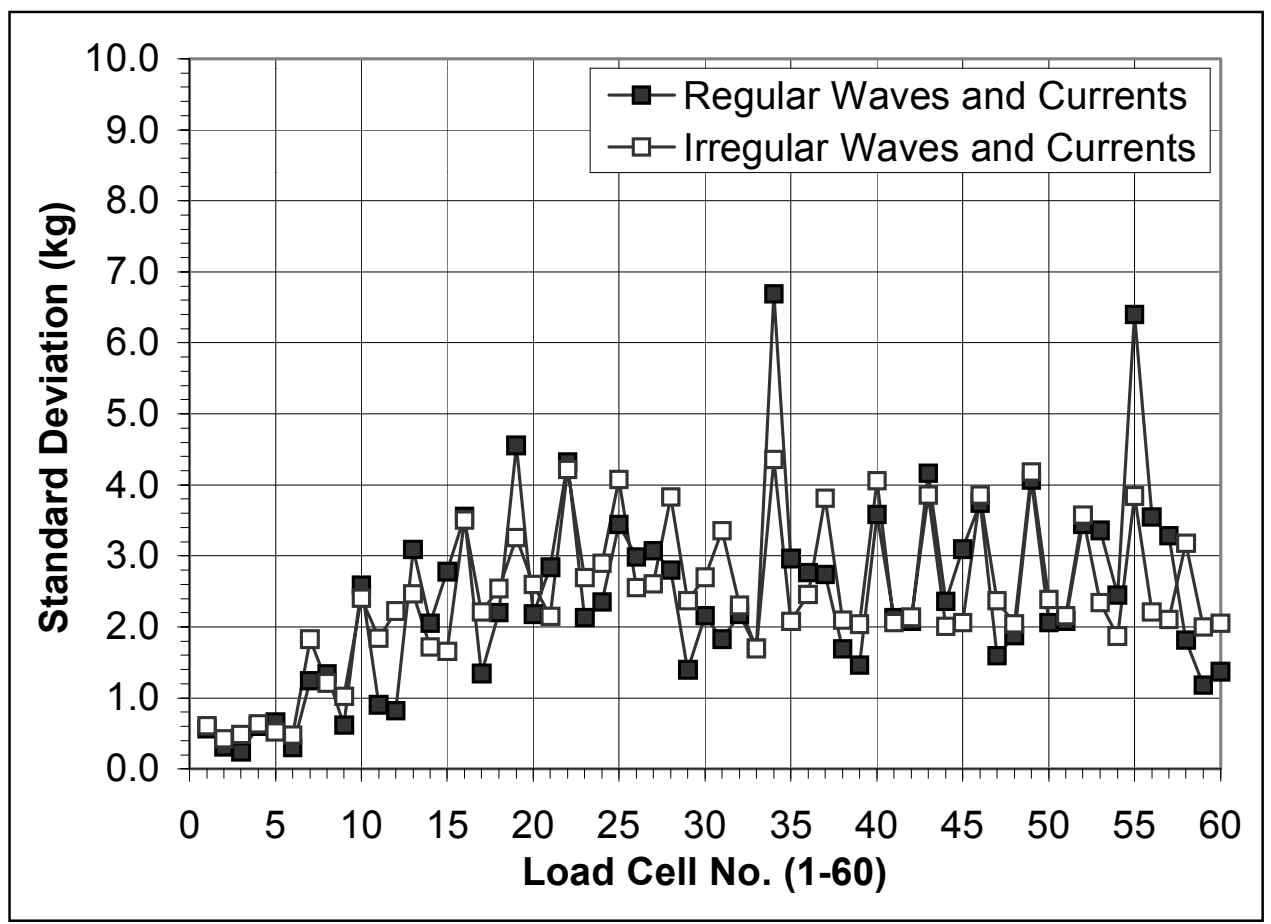

Figure 26. Standard deviation of load cell readings measured during dynamic performance tests with empty traps using both regular and irregular waves 


\section{Beach Morphology Control}

\section{Introduction}

The goal of the experiments is to obtain an accurate rate of longshore sediment transport and its cross-shore distribution with minimal longshore variation and boundary influences. To achieve this goal, it is necessary to maintain straight and parallel contours throughout the model. Beaches having "three-dimensionality" affect incident waves and, subsequently, the longshore currents and sediment transport associated with the waves are also affected. Therefore, it was necessary to maintain straight and parallel contours throughout the model to the greatest degree possible. This chapter describes the method used to construct the beach morphology and maintain straight and parallel contours to maximize the length of beach over which longshore uniformity of waves and currents exist in the basin.

\section{Maintaining Straight and Parallel Contours}

Sand used in the initial experiments was fine quartz sand of uniform size, $\mathrm{D}_{50}=0.15 \mathrm{~mm}$. A total of approximately $125 \mathrm{~m}^{3}$ of sand was transported into the facility by means of a tractor (Figure 27) and placed on top of the fixed-bed concrete beach. The tractor is equipped with a front-end loader and rear boxblade, which are used to distribute sand along the beach (Figure 28).

Stainless steel rods mounted on the bridge at $1.0-\mathrm{m}$ intervals in the crossshore direction are used as elevation indicators to help maintain straight and parallel contours (Figure 29). The rods are lowered to the desired elevation at each cross-shore location and fixed in place by an anchoring collar. Moving the bridge along the facility leaves traces in the sand at the desired beach elevation, and indicate locations that require cutting or filling. The tractor's boxblade and front-end bucket are used in the longshore direction to scrape or fill sand to the necessary elevation indicated by the rod traces (Figure 30). After a segment of beach is cut or filled, it is necessary to check the elevations by again traversing the rods over the beach. Several iterations of checking elevations with the rods and grading the beach are required before the beach has acceptable uniformity. To avoid possible breakage or bending of the rods, the depth of sand the rods are dragged is limited to a few centimeters. Therefore, grading of the beach to an approximate elevation may be required before the rods are used to indicate the desired beach profile. Although most of the beach can be graded using the tractor, it is necessary to manually cut and fill portions of the beach between rod 


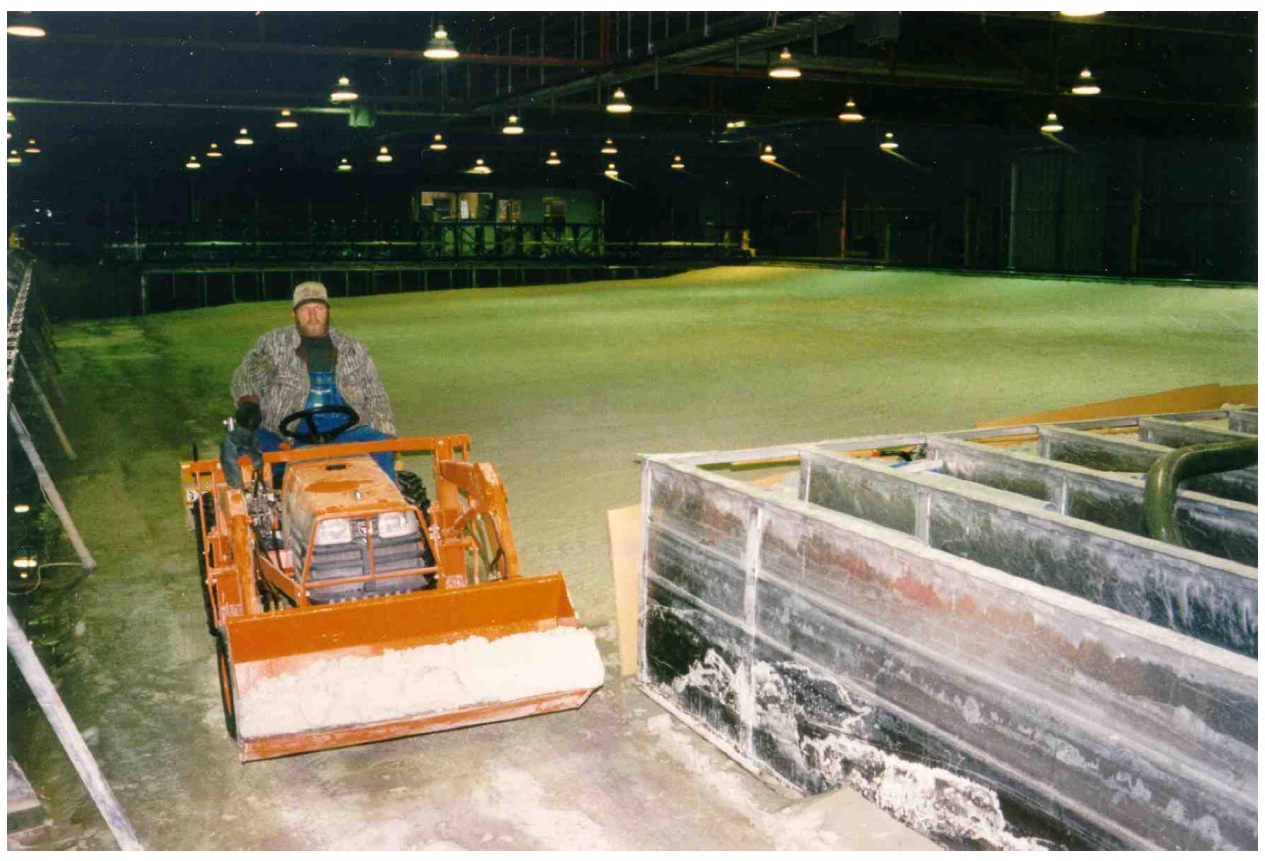

Figure 27. Sand is removed from a temporary storage area, located in the updrift, offshore corner of the facility. This compartment will hold 5 to $10 \mathrm{~m}^{3}$ of sand. If necessary, sand can be pumped from the sediment traps directly into this storage area during the dredging process. However, during a typical experiment, the sand in the traps is pumped directly onto the updrift end of the beach

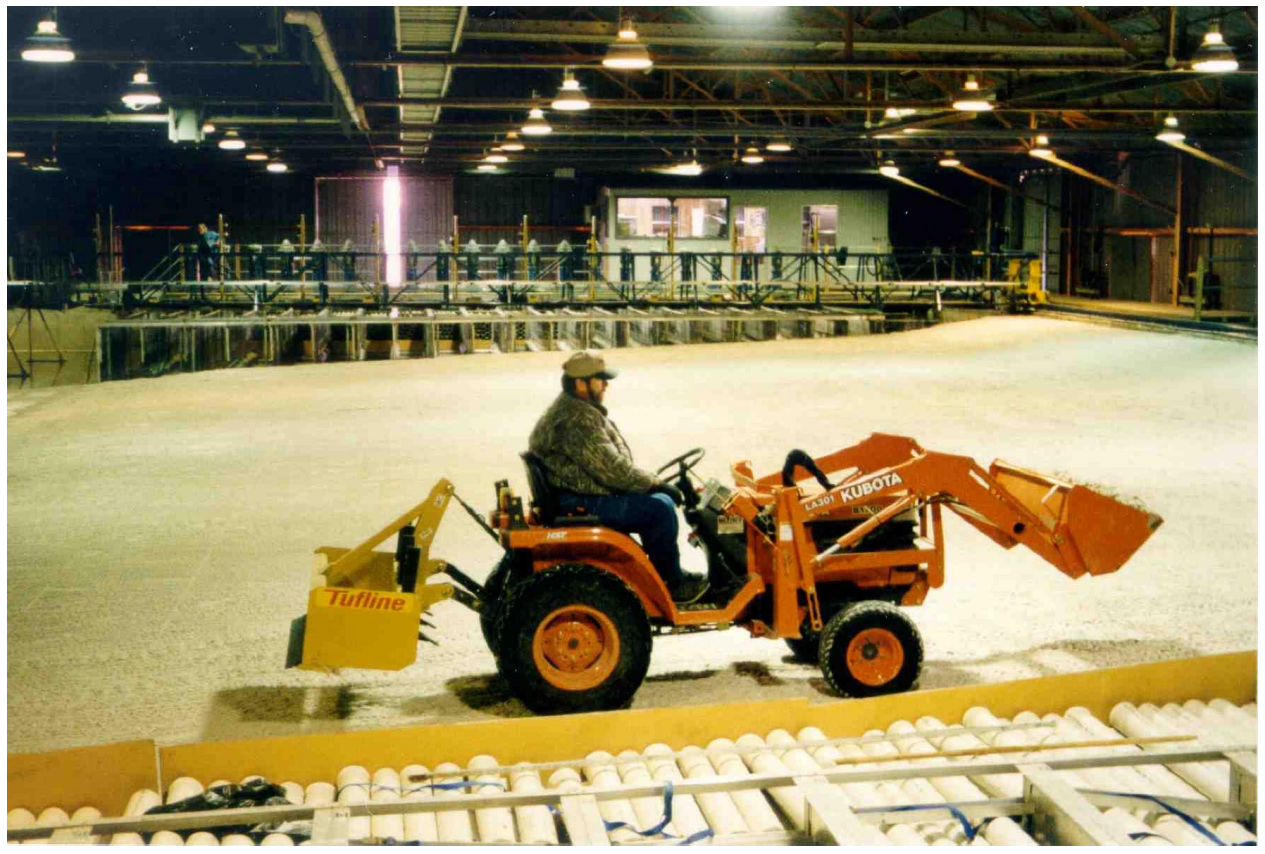

Figure 28. Prior to each experiment, the facility is drained, and a four-wheel drive tractor, with a front-end loader and a rear boxblade, is used to grade the sand beach to the desired beach profile shape 


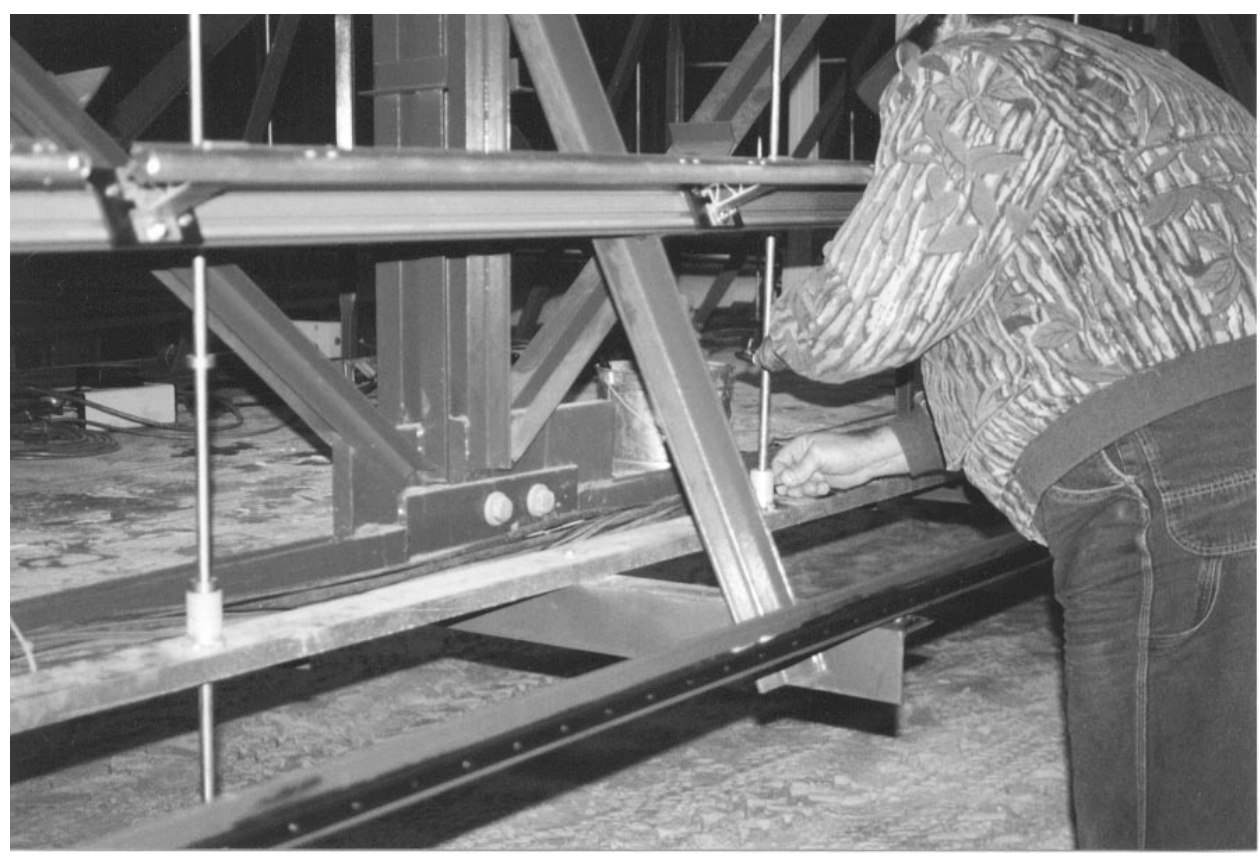

Figure 29. Stainless steel rods are permanently installed every $1.0 \mathrm{~m}$ along the updrift side of the bridge. The rods are lowered to preselected elevations to define the shape of the proper beach profile

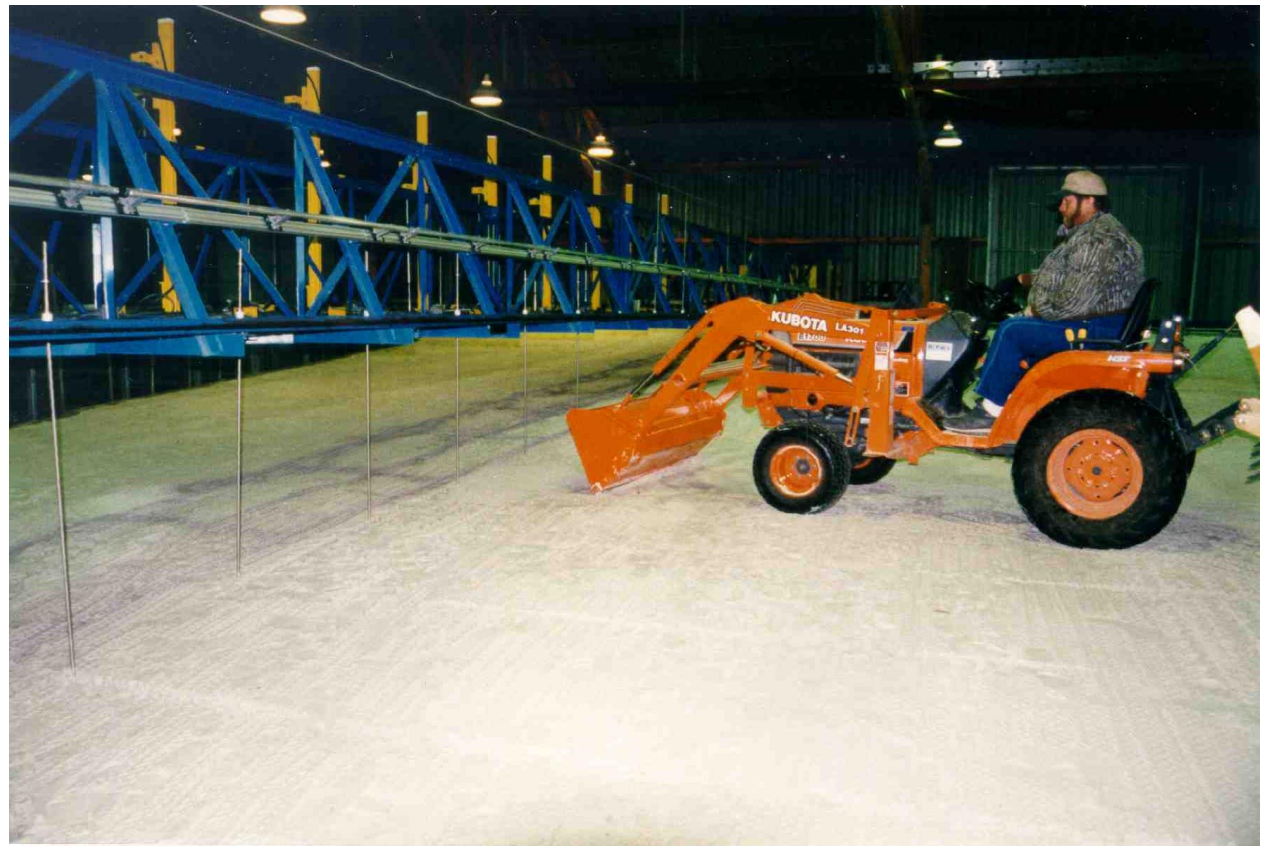

Figure 30. The vertical rods are used as indicators to help the tractor operator maintain straight and parallel beach contours. In this figure, the bridge is moving to the left, and the bottom end of the vertical rod furthest offshore is deflected to the right as it drags along the beach. The depth of the groove remaining in the sand indicates how much the beach should be lowered in that region 
traces in the cross-shore direction. Hand shovels and rakes are used to cut and fill in these areas. In addition, it is difficult to mold the beach in some regions to the desired elevation using only the tractor. Shovels and rakes are used to mold the steep foreshore slope or, if present, an offshore bar (Figure 31). The system of bridge-mounted rods used with the tractor and hand tools allows the elevation to be controlled in the longshore direction within $\pm 1 \mathrm{~cm}$. Figure 32 is a bathymetric plot of the beach after it had been remolded.

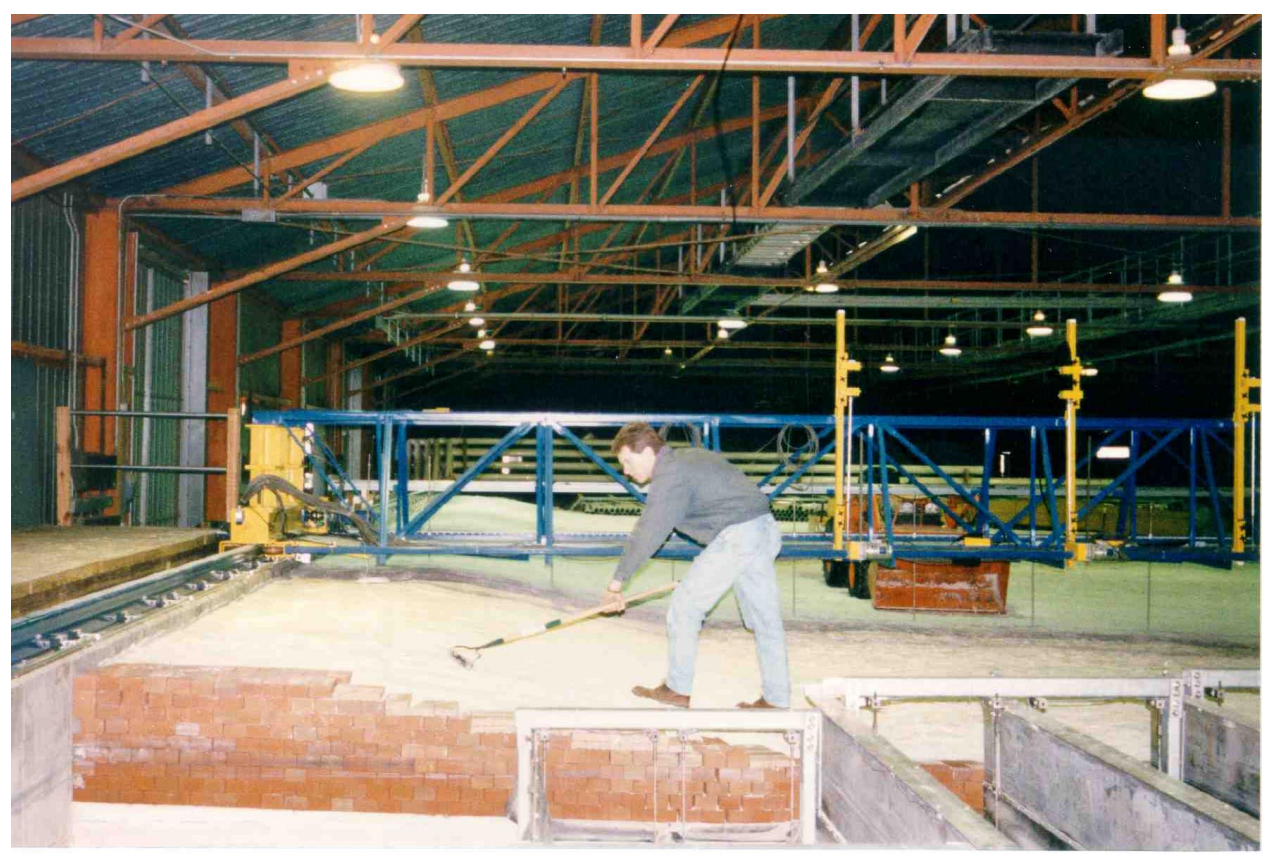

Figure 31. Fine-tuning of the initial beach profile is achieved using a hand held shovel and rake, especially in regions where it is difficult to use the tractor, such as the steep front slope of the foreshore berm

During the course of an experiment it becomes necessary to replenish the beach. As sand is trapped downdrift during an experiment, the updrift boundary becomes depleted of sand causing non-uniformity in the longshore direction (Figure 33). The purposes of beach replenishment are to recharge the sediment supply at the updrift end of the beach and to restore the beach to one with straight and parallel contours. Because the total amount of longshore sediment transport during individual wave runs is only a small fraction of the total amount of sand on the beach (typically less than 1 percent), continuous sand recharging during wave experiments is not necessary. The traps are dredged as described in Chapter 4 with sand being placed on the updrift portion of the beach. The main portion of the beach in the middle of the basin requires little attention in part to the uniform condition maintained by the longshore current recirculation system. The beach is rebuilt following the previously described method to the equilibrium, or quasiequilibrium, profile. During initial experiments, the beach evolves as it is subjected to waves until an equilibrium, or quasi-equilibrium, condition is established. It is desired to rebuild the beach to the profile the beach is seeking; therefore, the average beach profile measured along the uniform portion in the middle of the beach is used for rebuilding an evolving beach. 


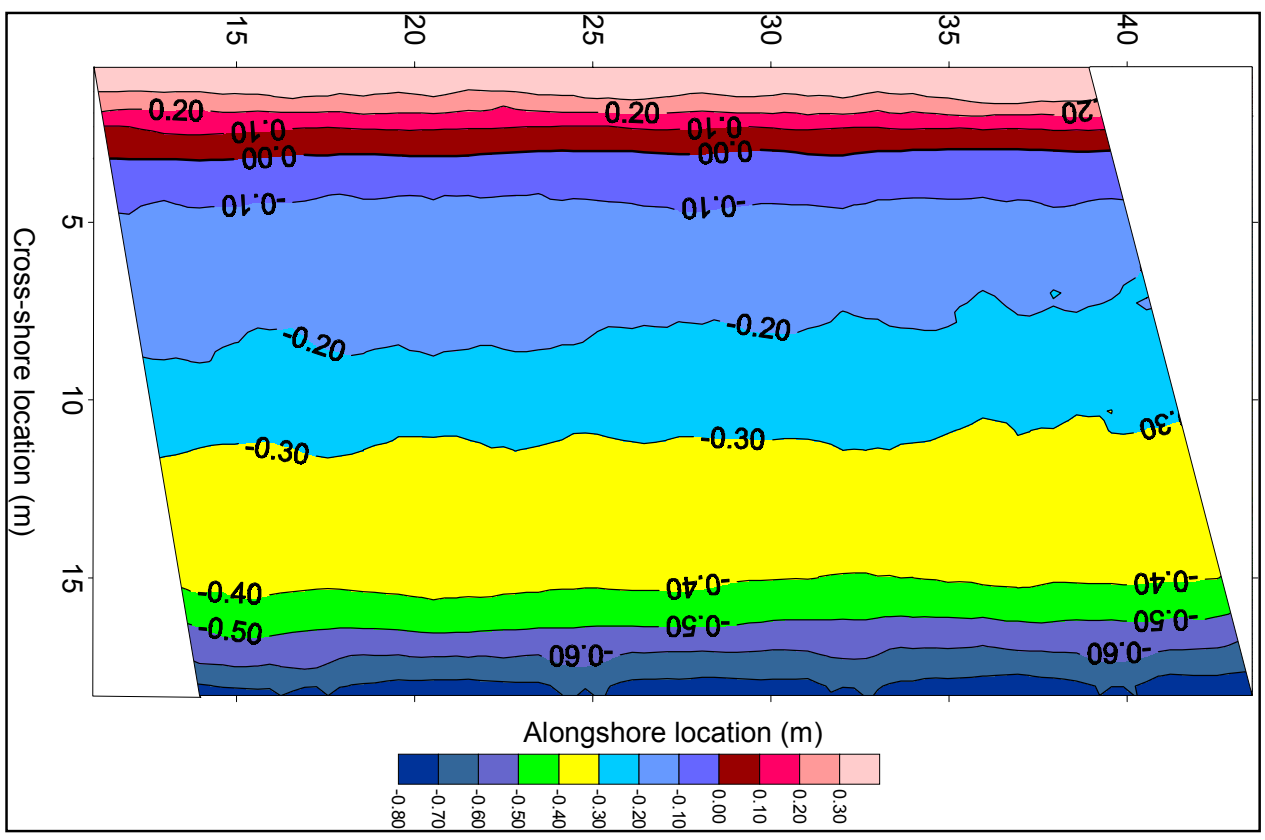

Figure 32. Bathymetric plot following rebuilding of the beach

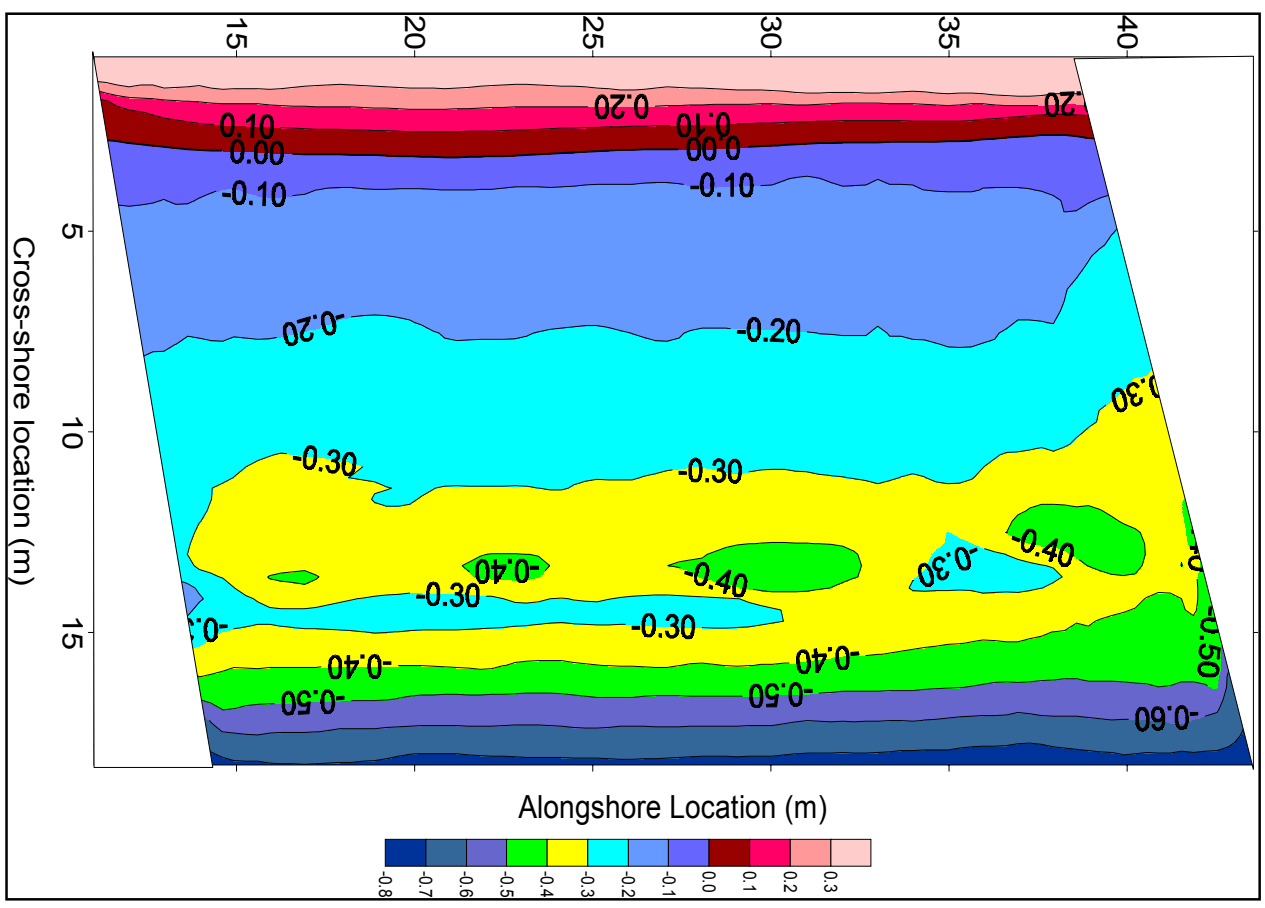

Figure 33. Bathymetric plot following $230 \mathrm{~min}$ of plunging waves

\section{Adjustable Boundary Elevation}

The lateral boundaries of the beach are bounded by stacked 19.5-cm-long by 9-cm-wide, 1.4-, 2.8-, and 5.6-cm-high mortar bricks placed on the fixed-bed concrete beach. The use of bricks of varying heights allows flexibility in 
constructing the boundaries similar to the average beach profile. Additionally, because of their density, mortar bricks are less likely to be displaced under waves and currents than other materials.

At the onset of an experiment, the bricks are arranged so that the boundary elevation closely matches the initial beach profile (Figure 34). It is important that the brick elevations at the downdrift boundary closely match the average profile of the beach. A lower brick elevation in relation to the beach may induce sand to enter the traps and cause an overestimate of sediment transport. Additionally, sand near the downdrift boundary scours to the upper elevation of the brick, which alters the local profile and uniformity of longshore currents. Conversely, if the brick elevation is higher than the beach elevation, the bricks act as a barrier and transport into the traps is restricted and results in underestimation of sediment transport. For this reason, the downdrift boundary is observed carefully during each experiment and, if necessary, bricks are removed or added so that the boundary profile is representative of the average beach profile.

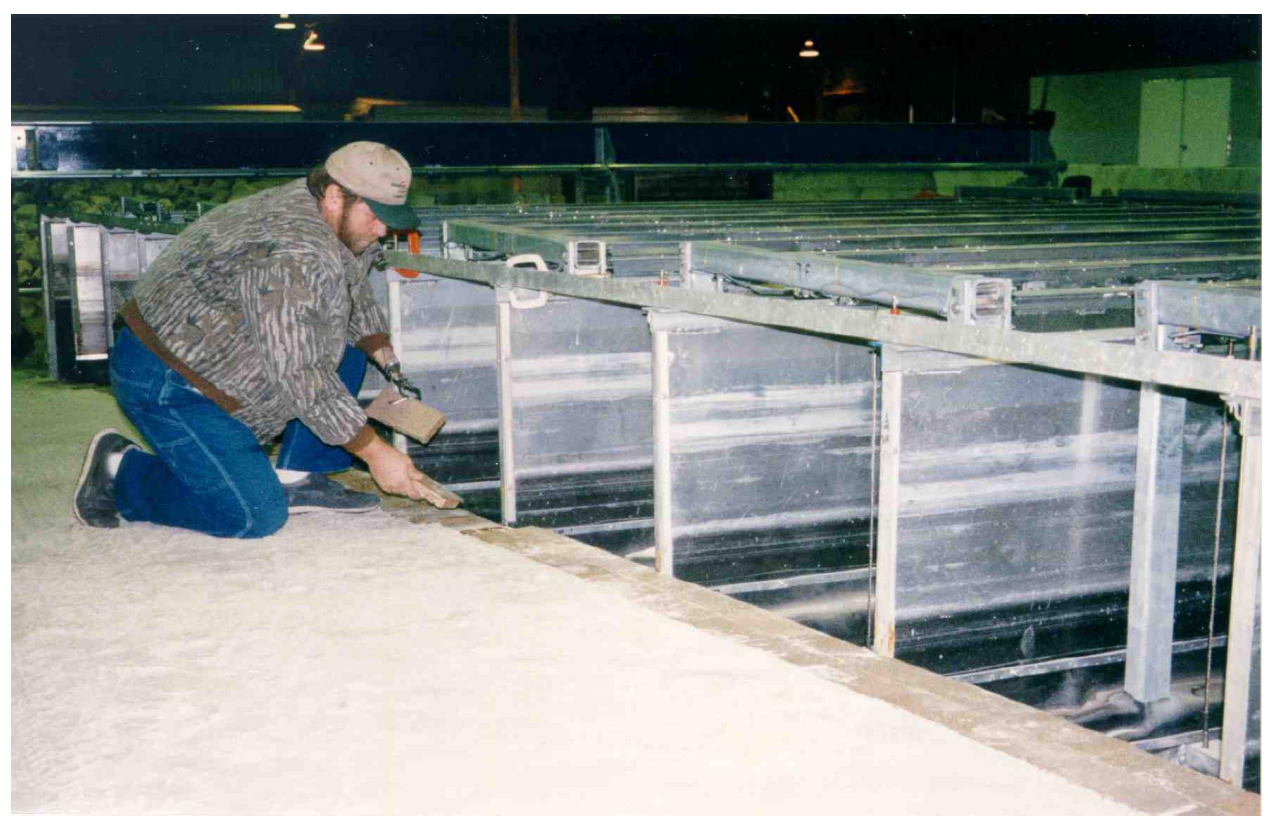

Figure 34. The elevation of the lateral boundaries is adjusted using bricks. Initially, the bricks are set as close as possible to match the initial beach profile. During the experiment, the downdrift boundary is observed carefully, and if necessary, bricks are removed or installed so that the downdrift brick boundary has almost the same profile shape as the average profile along the beach

Despite careful monitoring of the downdrift boundary to maintain the boundary elevation at the average profile elevation, not all transported sand reaches the traps. For mild to moderate longshore currents a shoal may form at the downdrift boundary, which must be included in calculations of longshore sediment transport. This correction is based on postexperiment surveys in which the region near the downdrift boundary is compared with the main portion of the beach. If a shoal is present, excess sand at the downdrift boundary is included 
with the sand measured in the traps to calculate longshore sediment transport rates.

\section{Summary}

The process of building the beach to straight and parallel contours is laborintensive, but necessary to obtain an accurate rate of longshore sediment transport. The use of a tractor and elevation indicators have been an effective and efficient method of accomplishing this goal. The masonry brick boundaries allow adjustment of the updrift and downdrift beach elevations to represent the average beach profile elevation. Beach uniformity is monitored from bathymetric surveys that follow each experiment segment. Additionally, the surveys monitor the existence of a shoal that may form at the downdrift boundary for mild or moderate longshore currents. The quantity of excess sand in the shoal is included in calculations of longshore sediment transport. 


\section{Experiment Control, Data Acquisition, and Analysis Packages}

\section{Introduction}

This chapter describes the various systems used for conducting experiments in the LSTF, including real-time experimental control, data acquisition, and analysis. The primary systems are:

a. MTS Systems Corporation control and data acquisition system.

b. General Experimental Control and Data Analysis Package (GEDAP) synthesis and analysis package.

c. Display system for the ADV digital data.

d. Control system for the vertical turbine pumps.

e. Data acquisition system for the flow meters.

f. Data acquisition system for the sediment traps.

This chapter provides a brief description of each system. For additional information, the reader is referred to the Users Manuals and Reference Manuals cited in each section.

\section{MTS Control and Data Acquisition System}

The MTS control and data acquisition system was developed as the primary system for operation of the facility and consists of two primary components. The first component is an MTS 498 real-time controller that uses closed-loop control to position the wave generators, the instrumentation bridge and the beach profile indicator. This system also contains the hardware required for calibration and data acquisition of the wave gauges, current meters, and the beach profile indicator. This real-time controller is a VME-based, multichannel, digital controller and contains a MVME 167 VME-based processor board, A/D and D/A converters, and optically isolated digital I/O. 
The second component consists of a personal computer that provides the operator with an interface for real-time control, data acquisition, data storage, and analysis. This $\mathrm{PC}$ has a $266-\mathrm{MHz}$ Pentium processor and uses the Windows NT operating system. The PC is connected to the real-time controller using a highspeed data link (MXI-bus interface). Application software was developed with a graphical user interface to be the primary user interface for operation of the facility. This application software is referred to as the "MTS control software."

The next four paragraphs provide a brief discussion of the various functions and organization of the MTS control software. To avoid confusion, these paragraphs are listed in the same order as the four pull-down menus located on the main panel of the MTS control software (Figure 35).

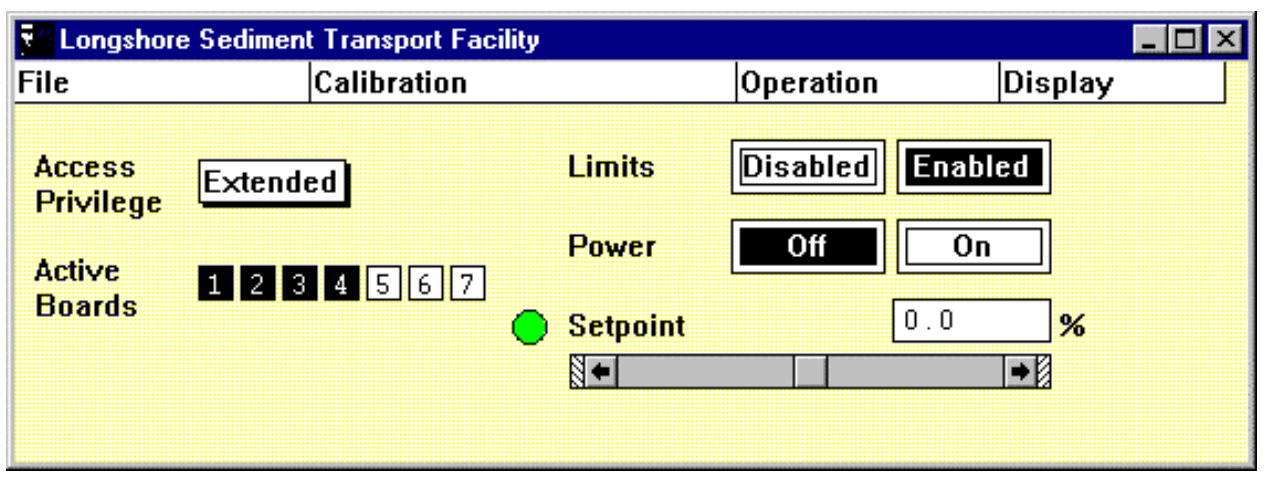

Figure 35. Main panel of the MTS control software (Permission to reprint granted by MTS Systems Corp.)

The File menu on the main panel of the MTS control software allows the user to restore and save the system parameter settings. A number of system parameters must be defined prior to conducting an experiment. These parameters require loading immediately after starting the MTS control software. Some system parameters only need defining during the initial installation of the equipment or after repair of the equipment, such as the servo control loop adjustments, numerical limit detector settings, and ramp rate settings. Other system parameters, such as those that define the calibration of the wave gauges, current meters, and the beach profile indicator require updating after every recalibration of these sensors. This information is saved in a "settings.set" file, to eliminate the need for redefining all of these system parameters at the beginning of each experiment, and also to maintain consistency between experiments. In addition, this menu provides options that allow the operator to select passwords, convert acquired data files to ASCII file format and to exit the MTS control software.

The Calibration menu on the main panel of the MTS control software provides access to control panels for calibrating the linear actuators, wave gauges, current meters, beach profile indicator, and other generic sensors that may be added in the future. The operator also can specify the number of sensors, which will configure each control panel for the number of sensors in use during an experiment. In addition, there are several other control panels for specifying other system parameters, such as the servo control loop adjustments, limit 
detector settings, Proportional-Integral-Derivative (PID) controller settings, wave program settings, ramp rates, and the engineering units associated with each input and output channel.

The Operation menu on the main panel of the MTS control software provides access to the control panels for operation and real-time control of the wave generators, instrumentation bridge, and the beach profile indicator. The beach profile indicator control panel allows the user to select all of the parameters required to conduct a fully automated beach profile survey, including the number and location of each transect and the horizontal speed of the beach profile indicator. Another control panel in this menu activates the data recorder, which allows the user to record (or collect) data from any or all analog and digital input and output channels. The most common channels to record are the analog output signals associated with the wave gauges and current meters.

The Display menu on the main panel of the MTS control software provides various options for displaying the analog and digital data, as well as error messages from the real-time control system. Two options provide access to two digital meters, which are the modern equivalent of a digital voltmeter. These digital meters are most useful for monitoring signals with a low frequency of oscillation, such as the position of the instrumentation bridge, or the still-water level recorded with the wave gauges prior to an experiment. Another option provides access to a digital oscilloscope, allowing the user to display the output signal from any of the analog or digital channels. The digital oscilloscope is most useful for monitoring output signals characterized by a higher frequency of oscillation, such as the output signal from the wave gauges or current meters, during operation the facility. Another control panel allows the user to read error messages generated by system events, such as error messages from the limit detectors.

A general description on how to operate the wave generators and instrumentation bridge, as well as a detailed discussion on how to use all of the control panels in the MTS control software, can be found in Chapter 4 of the Operation Manual, MTS Systems Corporation (1998).

\section{GEDAP Synthesis and Analysis Package}

GEDAP is a comprehensive software system for the analysis and management of laboratory data and includes real-time experiment control and data acquisition functions. GEDAP was designed with particular emphasis on random wave generation and analysis in hydraulics laboratory basins.

GEDAP is being used in the LSTF to perform the following tasks:

a. Calibrate the wave generators.

$b$. Synthesize wave machine drive signals for regular and random wave generation.

c. Synchronize the four piston-type wave generators when positioned at various angles of wave approach. 
d. Analyze measured wave data from the 14 capacitance-type wave gauges.

e. Analyze measured current data from the 10 ADVs.

GEDAP is being utilized in the LSTF, because it was designed with the following features:

a. A standard data file format so that any GEDAP program can process data generated by any other GEDAP program.

b. An extensive library of wave synthesis and data analysis programs to minimize the amount of project-specific programming.

c. A consistent user interface so that all programs are easy to use in either interactive or batch mode.

d. An extensive set of well-documented subroutine libraries so that future application programs can be developed quickly and efficiently.

e. A fully integrated graphics capability named GPLOT, to allow conveniently plotted at any stage of the data synthesis or analysis process.

f. A mechanism whereby data can be automatically identified whenever they are listed or plotted, and labeled with correct engineering units.

g. Conversion utilities that allow data transfer between GEDAP files and other standard formats, such as Microsoft Excel spreadsheets.

A block diagram of the basic GEDAP system is shown in Figure 36. This diagram shows the logical flow of data between the three different packages in the software, named NDAC, GPLOT, and ANALYSIS.

The NDAC package runs on Windows NT and performs real-time Data

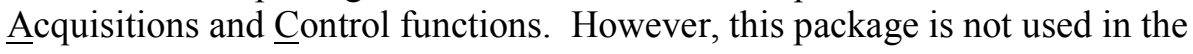
LSTF, because the MTS control software is used for this purpose. Therefore, to be more precise, the words "MTS Control Software" should be substituted for the acronym "NDAC" in the upper left corner of this diagram. A program named GEDAP_TO_MTS was developed to convert files from GEDAP to MTS file format, such as wave machine drive signals, which are synthesized using GEDAP. Another program named MTS_TO_DAC was developed to convert files from MTS to GEDAP file format, such as measured wave and current data files, which are analyzed using GEDAP. 


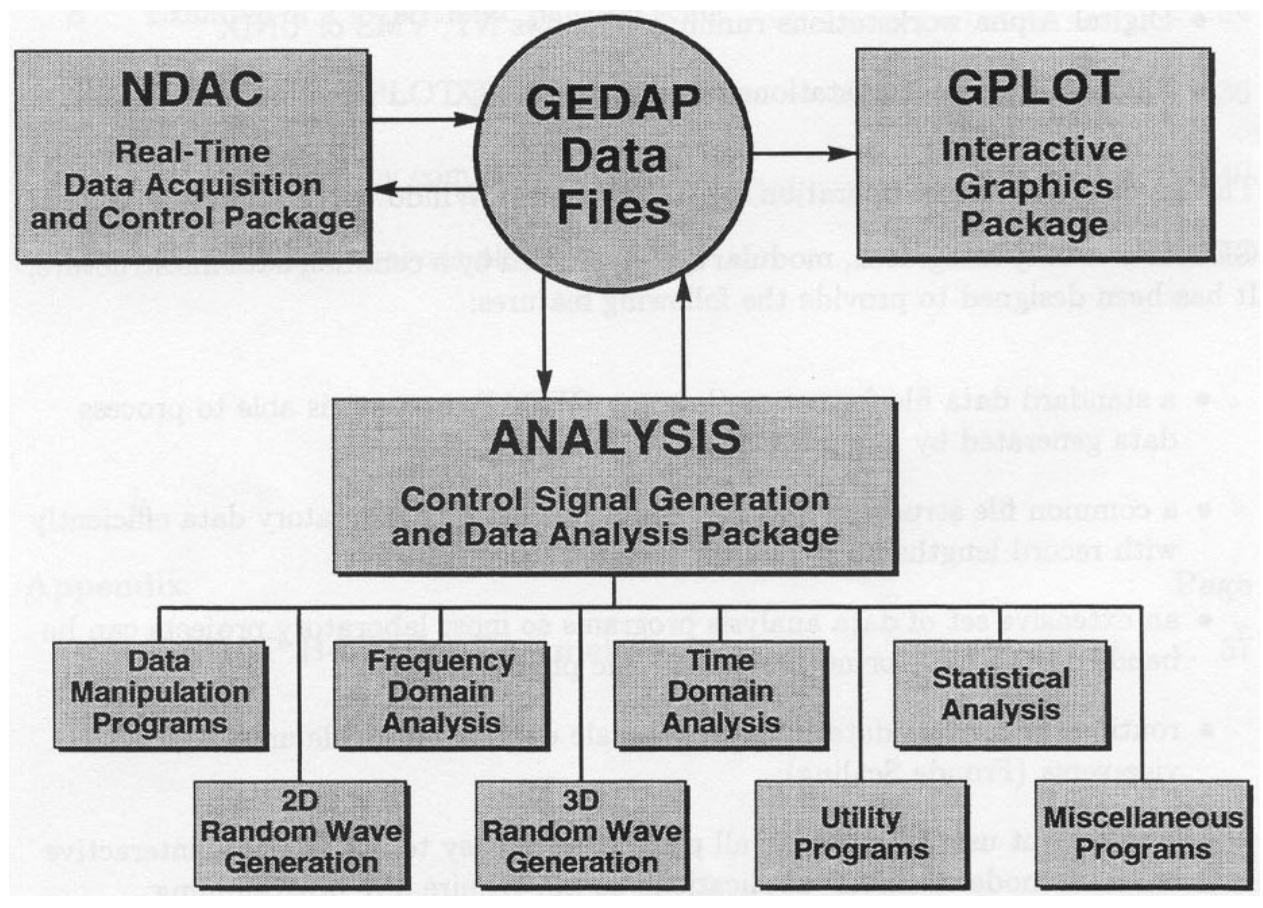

Figure 36. Block diagram of the GEDAP ${ }^{\mathrm{TM}}$ system of the Canadian Hydraulics Centre (Miles 1997) (Permission to reprint granted by Canadian Hydraulics Centre)

GPLOT is a comprehensive 2-D graphics package for plotting GEDAP data files. GPLOT is a command-driven program that can run in either interactive or batch mode. In interactive mode, the commands are entered directly on the keyboard. This mode is used primarily for viewing data and designing new plot layouts. In batch mode, the commands are read from GPLOT command files that have been previously created with a text editor. This mode is usually used for production runs. For example, in the LSTF several transects of wave gauge or current meter data can be analyzed as a production run. Fortunately, the GPLOT command files can be generated automatically while running in interactive mode, which greatly simplifies the task of designing plot layouts for production runs. All plots can be output to a local printer during both interactive and batch mode analysis.

The ANALYSIS package is the main part of the GEDAP software system that handles all of the data synthesis and analysis functions. This is a comprehensive package and is organized into eight categories, as shown at the bottom of Figure 36. The ANALYSIS package contains all of the GEDAP programs and subroutines except those in the NDAC and GPLOT packages. All of the programs used for regular and random wave synthesis, as well as synchronization of the wave boards in the LSTF, are located under the category entitled "2D Random Wave Generation." Similarly, all of the programs used for analysis of the wave and current data are located under the categories entitled "Frequency Domain Analysis," "Time Domain Analysis," and "Statistical Analysis." Batch files have been developed to: (a) synthesize wave machine drive signals and to synchronize the wave generators for both regular and random wave generation; (b) analyze a large number of transects of data from the 14 wave gauges; and (c) analyze a large number of transects of data from the 
10 current meters. The specific GEDAP programs used in each of these batch files will be discussed in the next two chapters.

The GEDAP software system runs in a standard Windows NT Command Prompt window. By typing "GHELP" at the command prompt, the user will obtain the main menu of the on-line documentation, as shown in Figure 37. This initial GHELP browser screen is split into three sections. The upper section includes two hyperlinks, one gives a general description of GEDAP and the other provides a complete list and description of all GPLOT commands. The middle section of this figure includes hyperlinks to the eight GEDAP program categories, which were represented earlier as the eight blocks at the bottom of Figure 36. The lower section includes hyperlinks that provide access to all of the subroutine libraries. The user can click on the highlighted hyperlinks to view lists of all of the available programs and subroutines. Similarly, the user can click on any highlighted program name to obtain a complete description of the particular program or subroutine.

Additional information about GEDAP may be obtained from the GEDAP Users Guide for Windows NT, Miles (1997). Additional information regarding the GPLOT graphics package can be found in the GPLOT User's Guide (Dominis Engineering Ltd. 1992).

\section{Display System for ADV Digital Data}

A problem encountered while procuring the ADV's for the LSTF was that the simultaneous digital output signals transmitted from the $10 \mathrm{ADVs}$ exceeded the capacity of the PC-based serial communication port. Therefore, each ADV was upgraded with additional hardware to generate analog output voltage signals, as an alternative means of collecting ADV data. The analog output voltages are transmitted through ten 130-m-long cables, to the MTS data acquisition and control system. However, the analog output signals only include the output voltage from each velocity component and one additional output signal, which is proportional to the mean signal amplitude. The analog output signals do not include all of the information that can be obtained using RS485 serial communication (digital data), such as the SNR for each velocity component, as well as other diagnostic information.

To solve this problem, a second computer is used in the LSTF control room for real-time display of the digital data obtained using RS485 serial communication. However, digital data can be displayed from only one ADV at a time, because of the data transfer rate limitations through the serial communications port. Therefore, the user must toggle from one ADV to the next to inspect the operation of each ADV, while conducting an experiment. The realtime display screen is shown in Figure 38. The upper section of the real-time display screen shows the numerical values of velocity and other diagnostic data, such as SNR, correlation, and the standard deviation for each component of velocity. The middle section of the screen shows real-time plots of SNR, correlation, and velocity data. The lower section of the screen lists all of the command options, such as a toggle switch, which allows the user to display the output from a different ADV. 


\begin{tabular}{||l|l|l|l|}
\hline File Edit Options Navigate Hotlists \\
\hline GI
\end{tabular}

Figure 37. On-line documentation listing the program classes and subroutine libraries of GEDAP ${ }^{\mathrm{TM}}$ system of the Canadian Hydraulics Centre (Miles 1997) (Permission to reprint granted by Canadian Hydraulics Centre)

This real-time display system is a completely independent system and is not integrated into the MTS Systems Corporation control and data acquisition system. The manufacturer of the ADV's developed the real-time display software. The procedures used for calibration, measurement, and analysis of the analog output signals, obtained from the ADV's, are discussed in Chapter 8 of this report. 


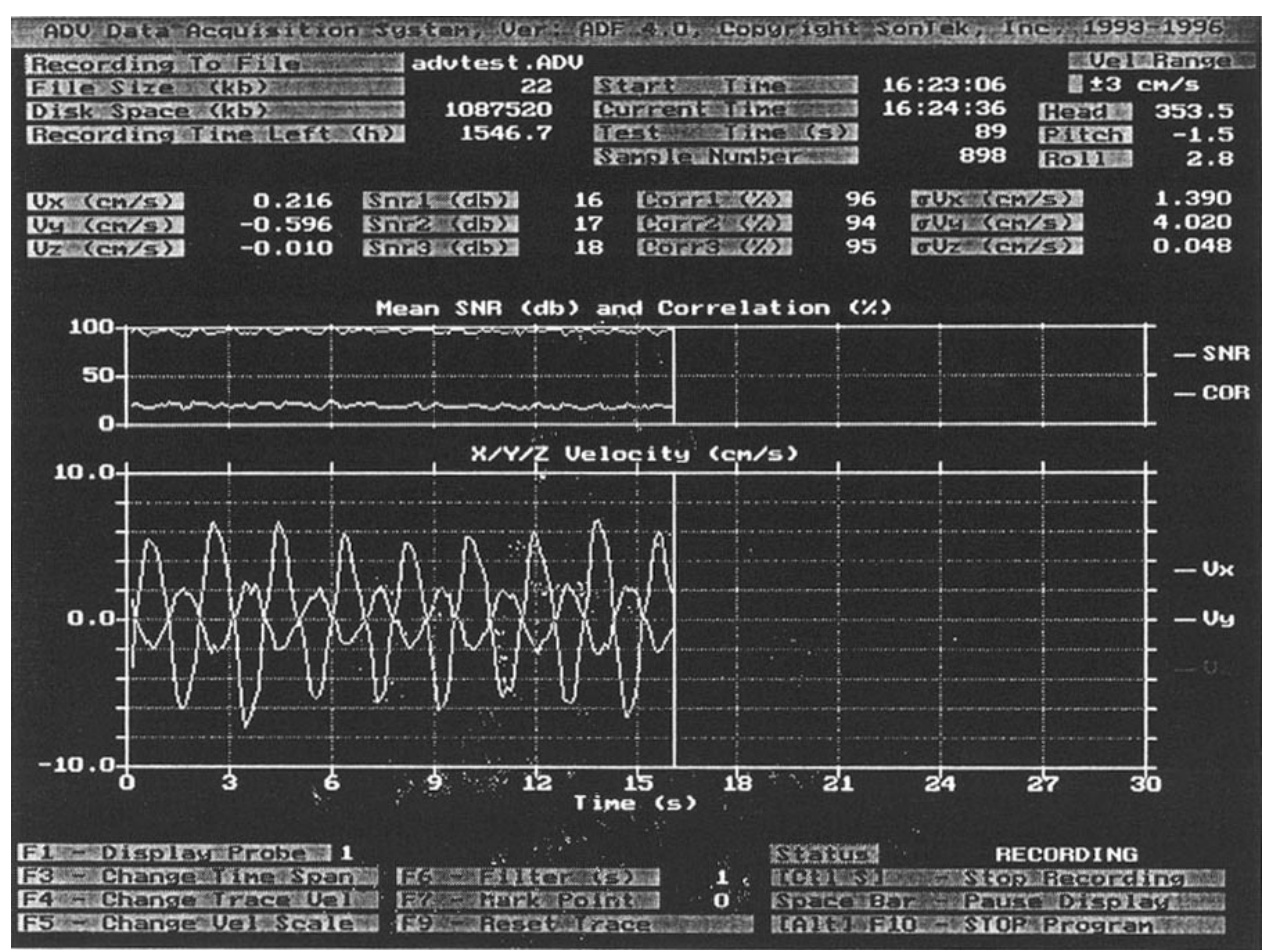

Figure 38. Real-time display screen for $A D V ®$ digital data (Permission to reprint granted by SonTek 1997) (SonTek and ADV are registered trademarks of SonTek/YSI Inc., San Diego, CA, USA)

\section{Control System for Vertical Turbine Pumps}

As discussed in Chapter 3 , the speed of the 20 vertical turbine pumps is controlled using 20 variable frequency motor controllers. These motor controllers are housed in a building adjacent to the LSTF control room. The standard input frequency to a motor controller is $60 \mathrm{~Hz}$. The speed of the pumps is controlled by adjusting the output frequency of the motor controllers, using a human interface module located on the front of each motor controller, shown previously in Figure 17. The output frequency can be adjusted from 30 to 100 percent of the input frequency (i.e., output frequency $=18$ to $60 \mathrm{~Hz}$ ). Because there is a linear relationship between output frequency, pump speed, and pump discharge, the discharge from the pump can be controlled from 30 to 100 percent of the maximum discharge rate. A detailed description of how to operate the motor controllers can be found in, Allen-Bradley (1996).

Each of the motor controllers were equipped with optional serial communication modules to provide a direct digital link between the 20 motor controllers and a personal computer. Control software could be developed that would allow the operator to enter the required discharge rates into the computer, and the software would calculate the output frequency for each motor controller based on the relationship between output frequency and pump discharge, previously shown in Figure 19. The control software would then adjust the output frequency of the motor controllers, based on the difference between the measured flow rate and the required flow rate. This would automate the present method of manually iterating to the required pump settings. Furthermore, using a 
personal computer to control the motor controllers would make it possible to vary the speed of the pumps during an experiment, for example to better approximate the time-varying longshore current magnitude generated from irregular wave forcing. This may be attempted in future experiments. However, at the present time, all pump speeds are constant during an experiment. The method of manually controlling the pump speed with the human interface modules has proven to require minimal time and effort. Therefore, the serial communication modules are not presently used. For further information on the serial communication modules, see Allen-Bradley (1995).

\section{Data Acquisition System for Flow Meters}

A third computer in the LSTF control room is dedicated to data acquisition and real-time display of data obtained from the in-line flow sensors. As discussed previously, these flow sensors are used to measure discharge rates from the 20 vertical turbine pumps in the longshore current recirculation system. This data acquisition system is a completely independent system and is not integrated into the MTS control and data acquisition system. The manufacturer of the flow sensors developed the software used for data acquisition and display.

The real-time display software allows the operator to visualize the crossshore distribution of flow rates through the 20 pump-and-piping systems. These data are displayed as a bar graph with Pumps No. 1 to 20 along the $\mathrm{x}$-axis and flow rate in liters per second along the y-axis. The flow rate measured with each sensor also is displayed numerically below each vertical bar in the graph. The data acquisition software was configured to output time-averaged values (every $30 \mathrm{sec}$ ) of the flow rate measured with each of the 20 flow sensors. The output file is an ASCII file consisting of 20 columns of data, one for each flow sensor. The files are then imported into a spreadsheet, and the average flow rate for the sampling interval (several minutes) is computed. The procedure used for obtaining the required pump settings using the manual iteration method, based on the measured flow sensor data, is discussed in Chapter 7.

\section{Data Acquisition System for Sediment Traps}

A fourth computer is dedicated to data acquisition and real-time display of data obtained from the sediment trapping system. Individual cables are used to connect the 60 load cells, which support the 20 sediment traps to the data acquisition system housed in the LSTF control room. This system is completely independent from the MTS control and data acquisition system.

The data acquisition and display software was developed at ERDC, via Visual Basic, to acquire and display measured data. The software includes two general options for displaying the data. The first option allows the operator to visualize the gradual increase (or trend) in the quantity of sand in a single trap. These data are displayed as an X-Y line plot, with time on the X-axis and weight on the y-axis. Four time series are plotted, the weight of sand recorded by each

of the three load cells and the total weight of sand in the trap. The second option 
allows the operator to visualize the cross distribution of the quantity of sand in the 20 traps. These data are plotted as a stacked bar graph with Traps No. 1 through 20 along the $\mathrm{x}$-axis and the submerged weight of sand along the y-axis. The height of the 20 bars represents the total submerged weight in each trap. A color-coded stacked-bar format was used to better visualize the distribution of weight on the three load cells in a given trap on one plot. Both options for visualizing the data can be displayed either in real-time or in postprocessing mode.

To minimize the size of the data files, the data are initially saved in binary format, and an option in the software can convert data into ASCII format. Two options for the structure of the ASCII output files are available. The first option outputs 60 columns of data, each representing the time series for each of the load cells. These files can be imported directly into a spreadsheet or analyzed using other programs. However, these output files are usually very large, since the sample duration can range from 1 to $2 \mathrm{hr}$ in length. The second option outputs only a summary of the standard statistical parameters that represent the 60 individual time series: average, minimum, maximum, and standard deviation for each time series. These files are considerably smaller. These statistical parameters are not very meaningful, however, if they represent the gradual increase in the quantity of sand in each trap during the experiment. Nonetheless, they are very useful if, for example, the quantity of sand in each trap was measured (for say $100 \mathrm{sec}$ at $1 \mathrm{~Hz}$ ) prior to and immediately after conducting the experiment, to verify the results obtained during the experiment. Options also are available for printing of the results. The procedures used for calibration, measurement, and analysis of these data are discussed in Chapter 8 of this report.

\section{Summary}

The LSTF is a complex facility and consists of many different types of equipment and instrumentation. With respect to real-time control of the equipment in the LSTF, 14 channels are presently being used; 8 command and feedback channels for the four wave generators, and 6 command and feedback channels for the three drive motors on the instrumentation bridge. In addition, the speed of the 20 vertical turbine pumps is accurately controlled by manually toggling the human interface module on the front face of each motor controller. With respect to data acquisition, 132 channels are presently being used; 14 wave gauge channels, 38 ADV channels, 20 in-line flow sensor channels to measure the discharge from the 20 pumps, and 60 load cell channels to measure the quantity of sand in the 20 traps.

The various real-time control systems, data acquisition systems, and analysis packages discussed in this chapter provide the operator with the capability to confidently manage all of this equipment and instrumentation, and therefore conduct a well controlled laboratory experiment in the LSTF. Experience has shown that all of these systems can be operated simultaneously by two operators. 


\section{Wave Generation and Current Recirculation}

\section{Introduction}

This chapter contains two major sections. The first section deals with the subject of generating waves in the facility, and specifically describes the procedures used to: (a) calibrate the wave generators; (b) synthesize regular and irregular wave drive signals; (c) synchronize the wave generators to create unidirectional long-crested waves; and (d) generate waves using a new drive signal. The second section deals with the subject of recirculating the longshore current using the longshore current recirculation system described in detail in Chapter 3. This section specifically describes the procedures used to:

(a) calculate the required pump discharge rates after the cross-shore distribution of the depth-average longshore current to be recirculated has been determined; and (b) set the speed of the pumps to achieve the required discharge rates from the 20 vertical turbine pumps. This chapter provides a conceptual overview of these procedures.

\section{Wave Generation}

All of the programs discussed in this section can be found in the "2D Random Wave Generation" category of the GEDAP analysis package. The location of this category with respect to the overall structure of the GEDAP system was shown previously in a block diagram in Figure 36. For several of the procedures discussed below, GEDAP batch files were created to simplify and standardize the procedure to maintain consistency from one experiment to the next.

\section{Calibration of wave generators}

The wave generators are calibrated by using a static calibration drive signal that is generated using program RWREP5, an acronym for Random Wave Reproduction. This calibration signal is used to slowly and smoothly move the four wave boards from zero to full forward stroke and then to full reverse stroke. The wave boards are held steady at the full forward, and the full reverse stroke 
positions for a period of $10 \mathrm{sec}$ to accurately measure the wave board displacement.

These calibration measurements are entered into program WMCAL, an acronym for Wave Machine Calibration, which produces a wave machine calibration file. The calibration drive signal includes an extra 2 -sec pause at 50 percent of full forward stroke. This pause is used to verify the polarity of the drive signals. If this extra pause occurs on the reverse stroke of the wave board instead of the forward stroke, the wave machine has reverse polarity. In this case, RWREP5 multiplies the drive signal by -1 so that the generated waves will have correct polarity and phase. After the static calibration is complete, all of the control parameters related to each particular wave machine can be obtained directly from the calibration file.

GEDAP also includes a method of dynamically calibrating the wave generators to compensate for the magnitude and phase lag of the wave boards at higher frequencies. This procedure was carried out initially, however, the results proved that the servo-electric drive systems of the four wave generators in the LSTF have very minimal magnitude and phase lag at higher frequencies. Therefore, only the static calibration procedure described above is required.

\section{Synthesis of regular wave drive signals}

A GEDAP batch file named REGULAR WAVE SYNTHESIS has been developed to synthesize regular wave drive signals. This batch file runs the program RWREP5, which includes the regular wave generation options: (a) the user specifies the wave height and the wave period; (b) the user specifies the wave height, and the program calculates the corresponding period for a wave of maximum steepness based on the breaking wave limit; and (c) the user specifies the wave period, and the program calculates the corresponding height for a wave of maximum steepness based on the breaking wave limit. Option $a$ is normally used for experiments in the LSTF. The program RWREP5 obtains calibration information for each wave generator from the four wave machine calibration files created using the program WMCAL. Four drive signal files are output from the program RWREP5, one to drive each of the wave generators.

\section{Synthesis of irregular wave drive signals}

A GEDAP batch file named IRREGULAR_WAVE_SYNTHESIS was developed to synthesize irregular wave machine drive signals. The general procedure consists of the following steps. First, the program PARSPEC, an acronym for Parametric Spectra, is used to synthesize a wave energy spectrum using one of the theoretical parametric models. The option for a Texel, Marsen, and Assloe (TMA) shallow water spectrum is normally used in the LSTF. Several input parameters need to be defined, including the water depth at the wave generators, the frequency of the wave spectrum peak, the peakedness factor of the spectrum, and the significant wave height. 
The program RWSYN, an acronym for Random Wave Synthesis, then is used to synthesize a pseudo-random wave train on the basis of a specified target spectrum obtained using PARSPEC. Each wave train consists of a time series record of wave elevation. Several input parameters require defining, including the model scale factor (normally $=1.0$ ) and the duration of the wave train. Typically, the duration of the wave train is normally set equal to $200 \times \mathrm{T}_{\mathrm{p}}$, the peak period of the wave spectrum. The length of the wave record is equal to the recycling period, the interval at which the control system automatically cycles back to the beginning of a time series when the MTS control software reaches the end of the time series. The wave train time series can be generated using one of three different methods, although the Random Phase Method is normally used. In this technique, the phases of the Fourier components are chosen at random, but the amplitudes are set deterministically according to the target spectrum. The resulting wave records thus have spectra that exactly match the target spectrum.

Next, the program RWREP5 is used to compute the wave machine drive signals to drive the four wave generators. These drive signals define the commanded position of the actuators (i.e., the position of the wave boards) of the wave machines, as a function of time. Several input parameters must be defined, including the water depth at the wave generators, the lower and upper cutoff frequency, and a wave amplification factor, WAF (usually WAF $=1.0$ ). If the

calibration process was carried out correctly, the measured wave height should be within 10 percent of the required wave height (with WAF $=1.0$ ). After the waves have been generated and measured, the wave amplification factor can be adjusted to either increase or decrease the generated wave height.

\section{Synchronization of wave generators}

The program RWREP5 also contains a subroutine to synchronize the four wave generators to create unidirectional long-crested waves with a total wave front $30.5 \mathrm{~m}$ in length. Four wave propagation distances are entered into the program RWREP5, one for each wave generator. The propagation distances are calculated based on the length of each wave board $(\mathrm{L}=7.64 \mathrm{~m})$ and the geometry of the layout of the wave boards. The propagation distance is the distance that a wave must propagate to be in phase with waves generated by the adjacent wave generator (travelling in the shoreward direction). This can be illustrated most effectively by referring to Figure 1 and by taking note of the layout and numbering of the wave generators. Waves generated from Wave Board No. 1 are closest to shore for all angles of incidence. If the boards are positioned at a 10-deg angle of incidence, relative to shore normal, Wave Board No. 2 is $1.347 \mathrm{~m}$ further offshore than wave board No. 1. Wave Board No. 3 is $2.694 \mathrm{~m}$ $(2 \times 1.347 \mathrm{~m})$ further offshore than Wave Board No. 1, and Wave Board No. 4 is $4.041 \mathrm{~m}(3 \times 1.347 \mathrm{~m})$ further offshore than Wave Board No. 1. In this case, the propagation distance is $1.347 \mathrm{~m}$. The drive signal for Wave Generator No. 1 is considered to be the master drive signal. Therefore, the drive signals for Wave Generators No. 2, 3, and 4 are a function of the master drive signal, and have the appropriate increase in phase at all wave frequencies to create unidirectional long-crested waves. Figure 4, shown in Chapter 2, shows the four wave generators creating unidirectional long-crested waves. This photograph was 
taken looking along the crest of the 30.5 -m-long wave front created by the synchronized generators.

For both of the above mentioned batch files, the last step in the synthesis process is to convert the four individual drive signals (initially in GEDAP file format) into a single file consisting of four arrays of data (MTS file format); one array for each of the four wave machine drive signals. Therefore, the final output from both of these batch files is a single file containing the synchronized drive signals for all four wave generators.

\section{Generating waves}

Waves are generated using the wave machine drive signal file in the Wave Programs control panel, found under the Operation menu of the MTS control software. The layout of this control panel is shown in Figure 39. The operator can select the length of time for which waves will be generated by selecting the desired number of times the file should be repeated.

The operator should visually observe and listen to the wave generators for the full duration of the drive signal when a new drive signal is initially used to generate waves, especially irregular waves. The operator should visually check if the wave generators are properly synchronized, as illustrated previously in Figure 5. If they are not, one or more of the parameters discussed above may be defined incorrectly. Another common problem is vibrations of the wave generators (i.e., mechanical "clunking"), which may be heard at high-frequency wave periods. In some cases, these vibrations can be very severe. This not only decreases the quality of the wave field but will also cause damage to the wave generators if allowed to operate for extended periods of time. This problem is normally caused by generating waves with a frequency too high relative to the magnitude of the wave height. For the case of irregular waves, the upper cutoff frequency, an input parameter in the program RWREP5, should not exceed approximately 1.5 to $2.0 \mathrm{~Hz}$ for the case of irregular waves. Selection of the proper upper cut-off frequency is normally determined by trial and error.

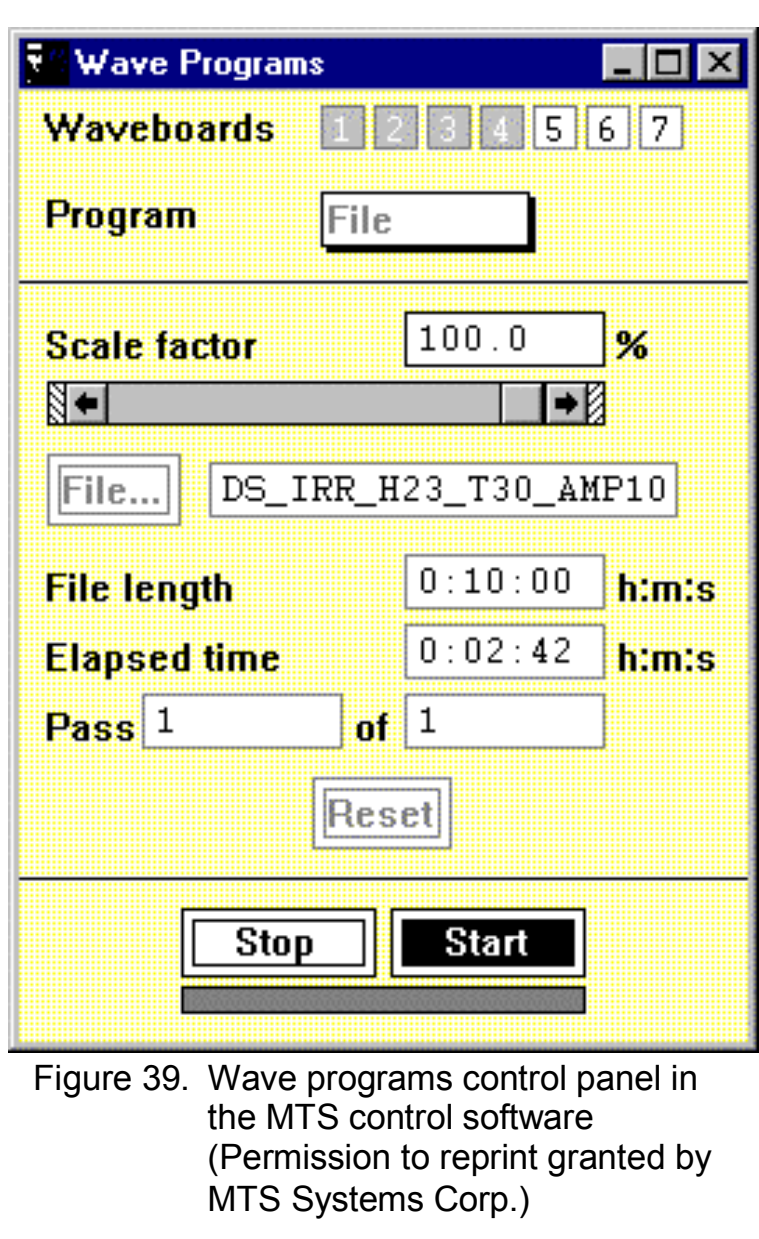




\section{Current Recirculation}

This section discusses the method used to calculate the required discharge rates of the pumps to recirculate the desired cross-shore distribution of the mean longshore current. The procedure used to estimate this cross-shore distribution will be discussed in detail in Chapter 9, and is based on the longshore current data measured at several transects along the beach during the previous segment of the experiment.

\section{Calculating pump discharge rates}

The method used to calculate the required discharge rates for the 20 vertical turbine pumps is illustrated in Table 3. These data used in the calculations were taken from a longshore sediment transport experiment.

Column 1 lists the pump number (or flow channel number) starting at Pump No. 1 (most shoreward) and ending with Pump No. 20 (farthest offshore). Column 2 lists the cross-shore position of the center line of each flow channel, with respect to the coordinate system used in the facility. A plan view of the layout of the pumps in the LSTF was shown previously in Figure 1, and an oblique view, looking in the offshore direction, was shown in Figure 16.

Water depth is shown in Column 3, measured from the still-water level to the elevation of the initial sand beach profile, which was mechanically graded using a four-wheel drive tractor prior to the experiment. This initial beach profile was shown previously in Figure 4. Depths listed in this column were measured along the beach profile at the center line of each flow channel.

Column 4 lists the average beach profile depth in front of each flow channel, based on the beach survey conducted immediately following the completion of the previous test segment. These values were measured relative to the still-water level. Each depth listed is the average value of 150 data points, obtained by measuring the elevation of the sand bed every $0.005 \mathrm{~m}$ along the beach profile transects. In addition, the values listed in column 4 are longshore averaged values based on six standard beach profile transects obtained using the beach profile indicator; namely $\mathrm{Y}=18,22,26,30,34$ and $38 \mathrm{~m}$.

Column 5 is the ratio of Column 4 divided by Column 3 and indicates the longshore averaged change in the most recently measured beach profile depth relative to the initial beach profile depth in front of each flow channel. For example, in this experiment the water depth in front of Flow Channel No. 1 had increased by approximately 50 percent relative to the initial beach profile depth. This indicates that the beach profile in that region (i.e., in front of Flow Channel No. 1) had eroded relative to the elevation of the initial beach profile in that region. In a similar manner, the water depth in front of Flow Channel No. 16 had decreased (accretion) by approximately 10 percent. This was caused by the formation of the offshore bar in that region of the beach profile. 


\begin{tabular}{|c|c|c|c|c|c|c|c|c|c|}
\hline \multicolumn{10}{|c|}{$\begin{array}{l}\text { Table } 3 \\
\text { Calculation of Discharge Rates for } 20 \text { Vertical Turbine Pumps }\end{array}$} \\
\hline $\begin{array}{l}(1) \\
\text { Pump or } \\
\text { Flow } \\
\text { Channel } \\
\text { No. }\end{array}$ & $\begin{array}{l}(2) \\
\text { Center } \\
\text { line } \\
\text { X-Loc. } \\
\text { m }\end{array}$ & $\begin{array}{l}\text { (3) } \\
\text { Initial } \\
\text { Beach } \\
\text { Profile } \\
\text { Depth } \\
\text { m } \\
\end{array}$ & $\begin{array}{l}(4) \\
\text { Measured } \\
\text { Average } \\
\text { Profile } \\
\text { Depth } \\
\mathrm{m} \\
\end{array}$ & $\begin{array}{l}\text { (5) } \\
\text { Ratio } \\
-\end{array}$ & $\begin{array}{l}(6) \\
\text { Measured } \\
\text { MWL } \\
\text { m }\end{array}$ & $\begin{array}{l}(7) \\
\text { Measured } \\
\text { Mean } \\
\text { Water } \\
\text { Depth } \\
\text { m } \\
\end{array}$ & $\begin{array}{l}(8) \\
\text { Measured } \\
\text { Average } \\
\text { c/s Area } \\
\mathrm{m}^{2}\end{array}$ & $\begin{array}{l}\text { (9) } \\
\text { Target } \\
\text { LSC } \\
\mathrm{m} / \mathrm{sec}\end{array}$ & $\begin{array}{l}(10) \\
\text { Pump } \\
\text { Discharge } \\
\text { Rates } \\
\text { l/sec }\end{array}$ \\
\hline 1 & 3.375 & 0.014 & 0.021 & 1.505 & 0.010 & 0.031 & 0.023 & 0.100 & 2.322 \\
\hline 2 & 4.125 & 0.042 & 0.068 & 1.627 & 0.010 & 0.078 & 0.059 & 0.135 & 7.928 \\
\hline 3 & 4.875 & 0.070 & 0.097 & 1.388 & 0.008 & 0.105 & 0.079 & 0.143 & 11.227 \\
\hline 4 & 5.625 & 0.098 & 0.130 & 1.320 & 0.006 & 0.135 & 0.101 & 0.150 & 15.205 \\
\hline 5 & 6.375 & 0.126 & 0.155 & 1.228 & 0.005 & 0.160 & 0.120 & 0.145 & 17.356 \\
\hline 6 & 7.125 & 0.154 & 0.172 & 1.118 & 0.004 & 0.176 & 0.132 & 0.135 & 17.826 \\
\hline 7 & 7.875 & 0.182 & 0.188 & 1.030 & 0.002 & 0.190 & 0.143 & 0.123 & 17.468 \\
\hline 8 & 8.625 & 0.210 & 0.207 & 0.986 & 0.001 & 0.208 & 0.156 & 0.115 & 17.976 \\
\hline 9 & 9.375 & 0.238 & 0.235 & 0.985 & 0.000 & 0.234 & 0.176 & 0.113 & 19.767 \\
\hline 10 & 10.125 & 0.266 & 0.262 & 0.985 & -0.002 & 0.260 & 0.195 & 0.115 & 22.455 \\
\hline 11 & 10.875 & 0.294 & 0.289 & 0.983 & -0.002 & 0.287 & 0.216 & 0.120 & 25.869 \\
\hline 12 & 11.625 & 0.322 & 0.317 & 0.984 & -0.002 & 0.316 & 0.237 & 0.120 & 28.401 \\
\hline 13 & 12.375 & 0.350 & 0.344 & 0.980 & -0.002 & 0.342 & 0.256 & 0.105 & 26.925 \\
\hline 14 & 13.125 & 0.379 & 0.362 & 0.956 & -0.002 & 0.360 & 0.270 & 0.080 & 21.595 \\
\hline 15 & 13.875 & 0.407 & 0.372 & 0.916 & -0.003 & 0.369 & 0.277 & 0.060 & 16.608 \\
\hline 16 & 14.625 & 0.435 & 0.393 & 0.903 & -0.005 & 0.388 & 0.291 & 0.040 & 11.627 \\
\hline 17 & 15.375 & 0.463 & 0.435 & 0.941 & -0.002 & 0.433 & 0.325 & 0.025 & 8.118 \\
\hline 18 & 16.125 & 0.501 & 0.498 & 0.994 & 0.000 & 0.499 & 0.374 & 0.015 & 5.610 \\
\hline 19 & 16.875 & 0.594 & 0.581 & 0.977 & 0.000 & 0.581 & 0.436 & 0.010 & 4.356 \\
\hline 20 & 17.625 & 0.687 & 0.674 & 0.981 & 0.000 & 0.674 & 0.505 & 0.010 & 5.055 \\
\hline
\end{tabular}

Column 6 lists the longshore averaged elevation of the mean water level (MWL) as measured with the wave gauges at the six standard transects. These values are measured relative to the still-water level. Column 7 is the measured mean water depth, which was calculated by adding the values listed in Column 4 to those listed in Column 6.

Column 8 is the longshore averaged cross-sectional area of the water column in front of each flow channel (i.e., the cross-sectional area of the water column in front of each flow channel in which the longshore current flows downstream). These values are obtained by multiplying the values in Column 7 by a factor of $0.75 \mathrm{~m}$, which is the width of each flow channel.

Column 9 lists the cross-shore distribution of the depth-averaged longshore current, to be pumped through the lateral boundaries of the facility. These values are obtained using the procedure developed for establishing uniform longshore currents which is discussed in detail in Chapter 9.

Finally, Column 10 is the pump discharge rates, for each of the 20 vertical turbine pumps, required to recirculate the target cross-shore distribution of the mean longshore current through the lateral boundaries of the facility. The pump discharge rates are obtained in liters/second (from cubic meters/second) by multiplying the product of the values in Column 8 and Column 9 by a factor of 
1,000. As shown at the bottom of the table, a total longshore flow rate of 303.7

$\ell / \mathrm{sec}$ will be actively recirculated during the next segment of the experiment.

It should be noted that Columns 3 and 5 are not required to calculate the pump discharge rates. However, they are always included in these calculations, because they provide a simple means of quantifying the change in beach profile depth in front of each flow channel (Column 5) relative to the initial beach profile depth (Column 3). This is an important parameter to quantify and monitor. For example, even though the target longshore current distribution (Column 9) may not be adjusted for the next segment of the experiment, the pump discharge rates may need to be adjusted slightly as the beach profile continues to make minor adjustments during later segments of the experiment.

\section{Setting the speed of the pumps}

There are several steps involved in setting the pumps to achieve the required discharge rates from the 20 vertical turbine pumps. First, the operator must determine whether to use the low-flow or the high-flow mechanism for each of the pump-and-piping systems discussed previously in the "Calibration and Testing" section of Chapter 3 .

Second, an estimate of the output frequency for each of the variable speed motor controllers is determined by using the calibration curves for each system. For example, the calibration curve for Pump-and-Piping System No. 10 was shown in Figure 19. As mentioned in Chapter 3, these calibration curves generally have an accuracy of within \pm 2 to 3 percent.

After the output frequency has been selected and the pumps have been turned on, the flow rate through each pump-and-piping system is measured using the inline flow sensors. Experience has shown that to obtain accurate and repeatable flow rates, the flow sensors must be measured for at least $500 \mathrm{sec}$ at a frequency of $20 \mathrm{~Hz}$. If the measured flow rate is less than the required flow rate, the output frequency of the motor controller is manually increased on the human interface module, shown previously in Figure 17. In contrast, if the measured flow rate is greater than the required flow rate, the output frequency is manually decreased on the human interface module. This process is repeated until all of the measured flow rates are within 1 percent of the desired flow rates for all 20 pumps. After the low-flow or high-flow mechanisms have been selected for each pump, the process of converging in on the correct frequency for the motor controllers requires approximately $30 \mathrm{~min}$.

During this 30-min period, the pumps force a longshore current along the moveable-bed beach. However, waves are not generated during this time. Fortunately, for most conditions the sand is stable under longshore current forcing only; i.e., the longshore current forcing alone does not usually generate sufficient near-bed velocity to exceed the critical shear stress required to initiate sediment transport for the sand particle size used in the LSTF. 
After the pumps have been set to achieve the desired cross-shore distribution of the depth-average longshore current at the lateral boundaries, an experiment can begin by generating waves.

\section{Summary}

Considerable experience has been obtained using the procedures described in this chapter. In regard to wave generation, GEDAP batch files were developed to synthesize regular and irregular wave machine drive signals to maintain consistency from one experiment to the next. Further information regarding the individual GEDAP programs can be obtained using the on-line documentation.

The procedure developed to calculate the required discharge rates for the 20 vertical turbine pumps to recirculate the desired magnitude and cross-shore distribution of the depth-average longshore current has proven to be accurate and dependable. This procedure takes into account the complexities involved in conducting sediment transport experiments with a moveable bed in which the beach profile shape changes as the waves and currents interact with the moveable bed. In addition, the method used to set the speed of the pumps using variable frequency motor controllers has proven to be very accurate and requires minimal time and human effort. 


\section{Sensor Calibration, Measurement, and Data Analysis}

\section{Introduction}

This chapter describes the procedures developed for calibration of the instrumentation in the LSTF, including the wave gauges, current meters, fiberoptic backscatter sensors, beach profile indicator, and the sediment traps. In addition, this chapter documents the procedures used for measurement and analysis of the five different types of data that are normally measured during a longshore sediment transport experiment in the LSTF. These data are: (a) time series of instantaneous water level fluctuations; (b) time series of instantaneous 3-D flow velocities; (c) time series of instantaneous suspended sediment concentrations; (d) bathymetric data of the moveable-bed beach; and (e) longshore sediment flux data. In addition, the method used to accurately control the water level in the facility will be discussed.

This chapter provides a significant amount of detail with respect to the specific steps involved in calibrating the instruments and conducting an experiment. In addition, many details related to the analysis of these data sets are provided, such as a description of the steps involved in the automated GEDAP procedures developed to analyze the wave and current data. These details should be useful for those involved in conducting experiments in the LSTF.

\section{Wave Data}

As mentioned previously in Chapter 2, the 14 single-wire capacitance wave gauges are used to measure instantaneous water level fluctuations (waves) in the facility. There are 10 wave gauges mounted on the instrumentation bridge and co-located with the current meters, as shown in Figure 40. In addition, four wave gauges are located offshore, along the toe of the moveable-bed beach, as shown with the facility drained in Figure B-5, Appendix B. These four gauges are centered in front of each of the four wave generators to measure the characteristics of the generated waves. 


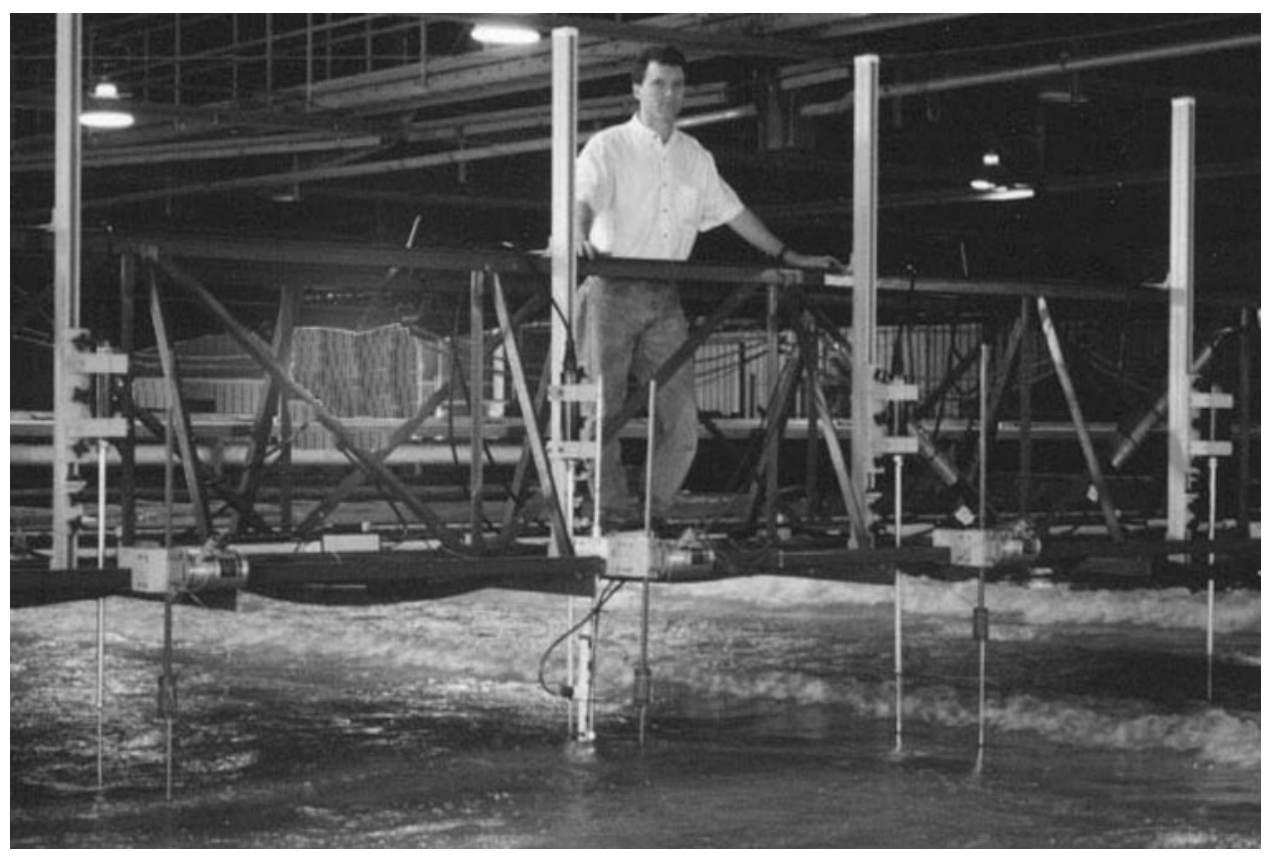

Figure 40. Measurement of wave, current, and sediment concentration data

The sensor portion of the capacitance wave gauge consists of a thin insulated wire held taut by a supporting rod. The rod is constructed of stainless steel with a minimum cross section to reduce flow disturbance. The wire insulation serves as a capacitor between the inside conducting wire and the water, which serves as the "ground". The capacitance varies linearly as the water surface elevation changes, provided the wire insulation thickness is uniform and not damaged, (Markle and Greer 1992). Advantages of the single-wire capacitance wave gauge are: (a) good linearity and dynamic response over a reasonable length that can be used for fairly large waves; and (b) the gauge is stable over sufficiently long time periods so that "gauge drift" is not a significant problem (Hughes 1993).

\section{Calibration of linear actuators}

Each wave gauge is mounted to a linear actuator that is used to raise and lower the gauge. The linear actuators can been seen in Figure B-4, Appendix B, and consist of an electric motor coupled to a rack-and-pinion mechanism which accurately raises and lowers the gauge. This allows for simultaneous remote static calibration of all wave gauges. Each linear actuator includes a high precision potentiometer that provides feedback for the position of the linear actuator. Calibrating these potentiometers is a semiautomated procedure and is partially accomplished using the MTS control software. The calibration procedure is carried out by manually positioning the linear actuator at approximately 10 elevations evenly spaced along the length of the actuator. At each elevation, the MTS control software automatically records the output voltage from the potentiometer. The elevation of the actuator is simultaneously measured using a high precision measuring device. This elevation is manually entered into the MTS control software, and this procedure is repeated for each elevation along the length of the linear actuator. After all positions have been 
recorded, the MTS control software determines the calibration equation based on regression analysis between the recorded potentiometer voltage outputs and the manually measured elevations. This procedure is repeated for each of the linear actuators.

The Linear Actuator Calibrations panel can be accessed under the Calibration menu of the MTS control software. These actuators have proven to have a very linear response, and a linear equation is normally representative of these data. This linear calibration equation is used to provide feedback for the elevation of the wave gauge, relative to the still-water level, during the automated calibration procedure for the wave gauges.

\section{Calibration of wave gauges}

Static calibration of the wave gauges is a fully automated procedure and is conducted remotely using the MTS control software. Experience has shown that dry wave rods will not provide high-quality calibration data. Therefore, the first step in the automated calibration procedure is to wet the wave gauges by temporarily submerging the entire length of each wave gauge. After this is complete, the linear actuators raise the gauges to locate the still-water level in the middle of the calibration range for each wave gauge. The gauges are automatically lowered in preset increments until the still-water level is located at the upper elevation of the calibration range. The gauge response is recorded at each elevation. Next, the gauges are automatically raised in preset increments until the still-water level is located at the lowest elevation of the calibration range. The gauges are automatically lowered in preset increments until the stillwater level reaches the midpoint of the calibration range for each gauge. This procedure can be conducted in approximately $30 \mathrm{~min}$, since the 14 wave gauges are calibrated simultaneously. The wave gauges are generally calibrated prior to each experiment.

The Wave Gauge Calibration panel, shown in Figure 41, can be accessed under the Calibration menu of the MTS control software. Both the linear and quadratic fit options are available in the MTS control software, as shown in the figure. It is sometimes prudent to use the second-order regression equation (quadratic) to increase the precision of the wave gauge calibration. 


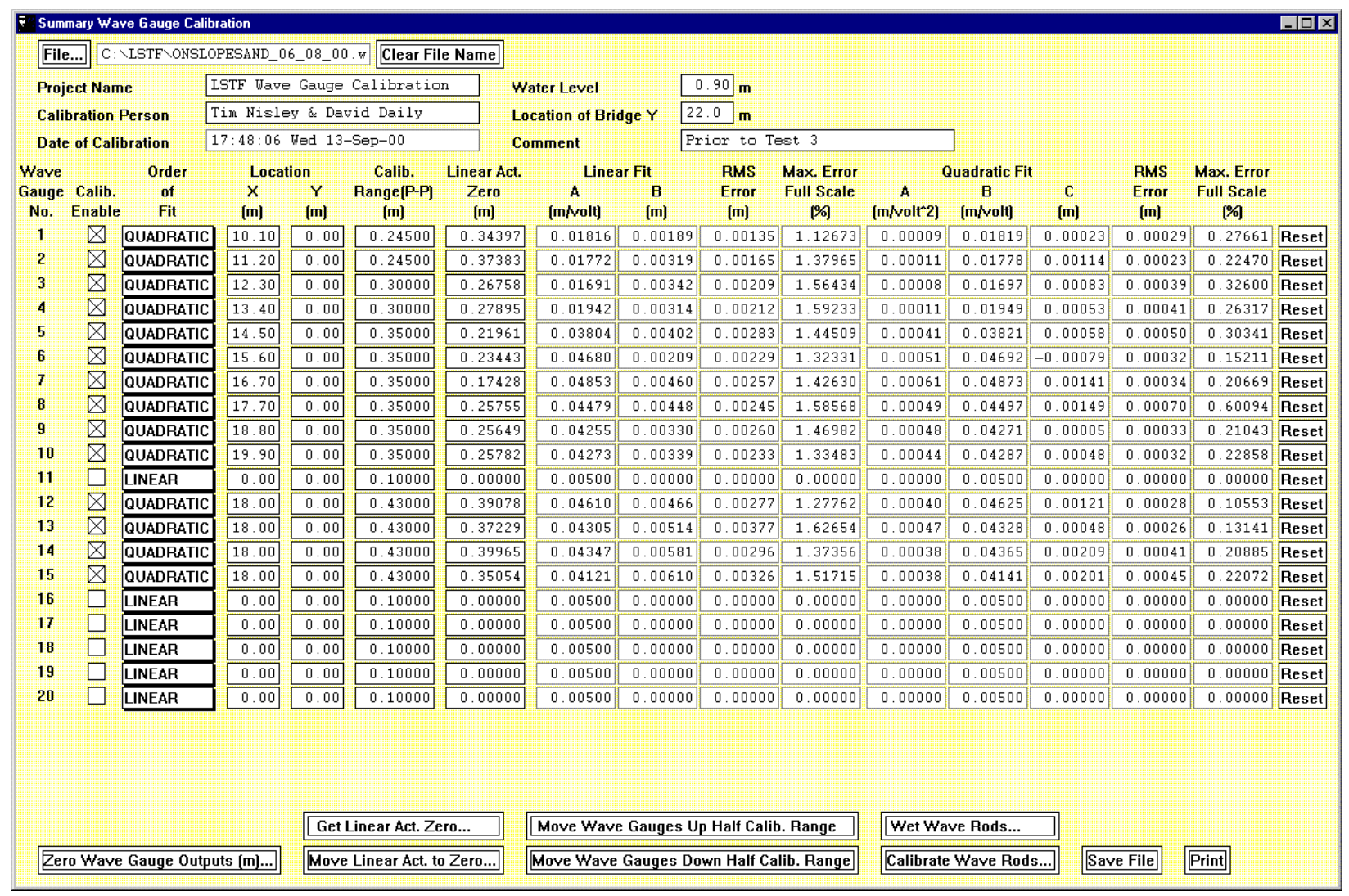

Figure 41. Summary panel of wave gauge calibrations in MTS control software (Permission to reprint granted by MTS Systems Corp.)

\section{Measurement of waves}

The wave gauges can be accurately positioned at any $x-y$ location within the testing region of the facility. The cross-shore position of the wave gauges can be adjusted at the beginning of the experiment by manually relocating the gauges along the length of the bridge. Figure 40 shows the wave gauges evenly distributed every $1.5 \mathrm{~m}$ along the bridge, so that 10 wave gauges cover a total range of $13.5 \mathrm{~m}$ in the cross-shore direction. However, rather than evenly distributing the wave gauges, it may be important to position a few gauges more densely in a certain region to provide sufficient cross-shore resolution to measure changes in the wave height gradient in response to the changing moveable-bed beach profile. Nonetheless, 10 wave gauges located along the length of the bridge have provided sufficient cross-shore resolution to accurately document the cross-shore distribution of wave height and mean water surface elevation.

During an experiment, waves are measured simultaneously with the 14 wave gauges at a frequency of $20 \mathrm{~Hz}$ to provide sufficient temporal resolution to accurately measure the profile shape of individual waves. For most wave conditions generated in the LSTF, the shortest wave period in the irregular wave spectrum is approximately $0.5 \mathrm{sec}$. Therefore, a sampling frequency of $20 \mathrm{~Hz}$ provides 10 data points to delineate the profile shape of these shorter period waves. 
The sampling duration is set equal to the duration of the wave machine drive signal for two reasons. The first being to ensure statistical wave height parameters obtained from the wave records are a realistic representation of the waves generated during the entire duration of the wave machine drive signal. Secondly, it provides the flexibility to start data acquisition at any time without synchronizing with the beginning of the wave machine drive signal, which allows measurements of the same set of generated waves during each transect.

The longshore position of the wave gauges is selected from the control room by automatically moving the instrumentation bridge to the desired transect location. The length of the test segment (generally 1 to $2 \mathrm{hr}$ ) limits the number of transects that are measured. For example, if the peak wave period is $2.5 \mathrm{sec}$, the duration of the wave machine drive signal is normally $500 \mathrm{sec}(200$ waves $\times$ $2.5 \mathrm{sec}$ ). Therefore, a sampling duration of $500 \mathrm{sec}$ is used. In addition, approximately $60 \mathrm{sec}$ is required to move the bridge to the next transect location. Hence, each transect would require approximately $10 \mathrm{~min}$ of testing time. Therefore, a 1-hr test segment provides time to measure approximately six transects, and a 2-hr test segment provides time to measure approximately 12 transects. The 12 transects distributed along the length of the testing region provide sufficient spatial resolution to quantify longshore uniformity (or longshore gradients) in wave height and mean water level.

Wave data are collected using the Data Recorder panel, located under the Operation menu of the MTS control software. The layout of this control panel is shown in Figure 42. The Data Recorder panel allows the operator to select the channels to record and stores data in a single binary file. During an experiment, the analog output signals from the 14 wave gauges and the 38 ADV channels are recorded using the MTS control software. The operator also can specify the sampling rate (i.e., sampling frequency) and recording period (i.e., sampling duration). Further information related to using the Data Recorder panel can be found in Chapter 4 of the MTS Operation Manual, MTS Systems Corporation (1998).

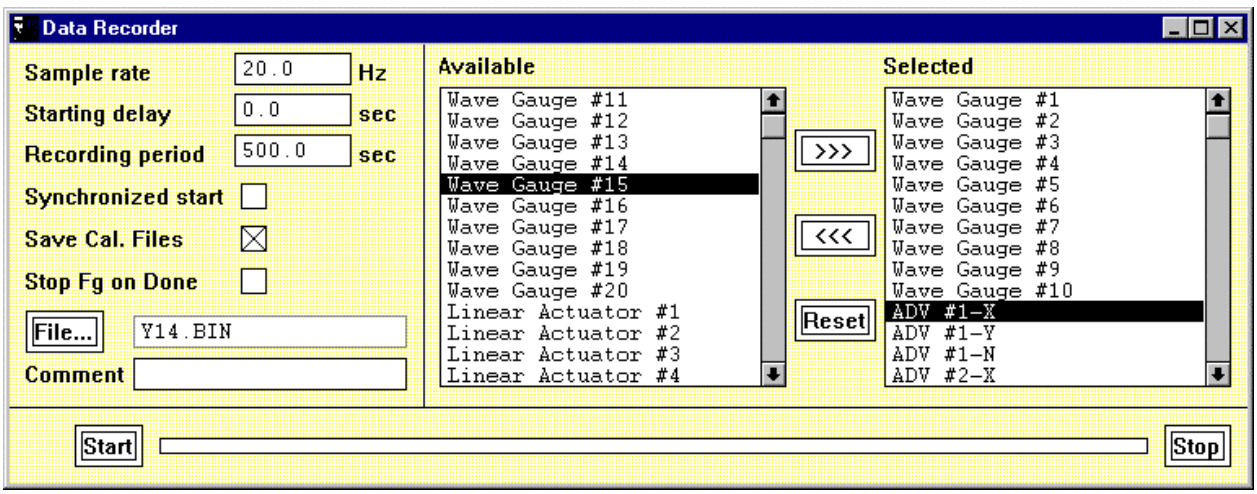

Figure 42. Data recorder panel in the MTS control software (Permission to reprint granted by MTS Systems Corp.) 


\section{Analysis of waves}

A GEDAP batch file was developed to automate the procedure of postprocessing several transects of wave data. The general procedure consists of the following steps. First, the program FILT_FFT is used to remove the lowfrequency wave energy in the surf zone which results from a slowly fluctuating mean water level. This step is especially important in shallow water near the shoreline. This program uses a rectangular bandpass filter to remove all wave energy that occurs at frequencies below $\mathrm{F} 1 \mathrm{~Hz}$ and at frequencies above $\mathrm{F} 2 \mathrm{~Hz}$. The fast Fourier transform (FFT) of the input signal is computed and the rectangular bandpass filter is applied in the frequency domain. An inverse FFT is used to obtain the filtered output signal. During recent experiments conducted using irregular waves with a peak frequency of $0.67 \mathrm{~Hz}, \mathrm{~F} 1=0.33 \mathrm{~Hz}$ was selected as the low-frequency energy cutoff. The upper cutoff frequency, F2, was set equal to the Nyquist frequency.

The program VSD, an acronym for $\underline{\text { Variance }} \underline{\text { Spectral }}$ Density analysis, is used to calculate the peak frequency and the energy-based significant wave height of the filtered output signal. The program ZCA, an acronym for Zero Crossing Analysis, also is used to calculate the average and maximum wave height as well as the average wave period of the filtered output signal. In addition, the program STAT1 is used to calculate the root-mean-square wave height and the mean water level for the filtered output signal.

Finally, the GEDAP program PLUCK is used to collect the corresponding values of the seven statistical parameters discussed above from the headers of each of the output files. The final result is an ASCII file consisting of 10 columns of data. The first three columns identify the wave gauge number and the $\mathrm{x}$ - and $\mathrm{y}$-locations. The last seven columns contain the values of the seven statistical parameters discussed above for the 14 wave gauges. As many as 12 transects have been measured during a 2-hr segment of an experiment. The ASCII file lists the statistical results obtained from the 12 transects, one transect below the other. All of the wave data can be analyzed in approximately $15 \mathrm{~min}$.

Examples of the cross-shore distribution of the measured wave height during a regular wave and irregular wave experiment are shown in Chapter 9. In addition, the cross-shore distribution of the measured mean water surface elevation for the same two experiments, are shown in Chapter 9, as well.

\section{Current Data}

As mentioned previously in Chapter 2, the 10 ADVs are used to measure 3-D flow velocities (currents) in the facility. The ADVs are mounted along the downstream side of the instrumentation bridge and are co-located with the wave gauges, as shown in Figure 40 and Figure B-4, Appendix B. The ADVs are configured to operate at $10 \mathrm{MHz}$ because measurements are required in shallow water and a high spatial resolution is needed to quantify the vertical velocity profile in shallow-water depths. 


\section{Calibration of ADVs}

Calibration of an ADV is based on the geometry of the acoustic transmitter and receiver as well as the speed of sound in water. Unlike capacitance wave gauges, ADVs do not require calibration on a regular basis. The manufacturer's calibrations give reliable results, provided that the acoustic transmitter and receivers are not damaged. The only parameter that influences the calibration of the ADVs is the speed of sound in water, which should be compensated for on a daily basis. Therefore, the water temperature in the facility is monitored daily and the temperature in degrees Celsius is entered into the ADV software prior to each experiment.

The calibrations can be verified by towing the ADVs through the water while driving the bridge along the rails at a constant speed. However, because the facility holds a large volume of water $\left(1,350 \mathrm{~m}^{3}\right.$, for a water depth of $\left.0.9 \mathrm{~m}\right)$, it is important to allow the water several hours to become completely quiescent before conducting these tests.

Custom designed 1.8-m-tall vertical struts and mounting devices were designed to make it easy to manually raise and lower the ADV's and to minimize the possibility of damage. Each vertical strut has an adjustable rubber bumper that can be positioned at the appropriate elevation so that if the ADV is dropped while being manually raised or lowered, the instrument would stop at the elevation of the bumper, rather than driving the ADV sensor into the beach. Considerable care is shown when moving the ADVs to ensure that the orientation of the sensors is maintained and that the acoustic transmitter and receivers are not damaged.

\section{Calibration of analog signals}

As mentioned previously in Chapter 6, the serial communications port does not have sufficient capacity to simultaneously transmit digital output signals from 10 ADVs at a frequency of $20 \mathrm{~Hz}$. Therefore, the digital data obtained from the ADVs is used only for real-time display of single ADV channels. Each ADV was upgraded with additional hardware to generate analog output voltages, which are transmitted through ten 130-m-long cables, to the MTS control and data acquisition system. The analog output voltage from the ADVs ranges from 0 to $5 \mathrm{~V}$ and is equivalent to a velocity range of $+100 \mathrm{~cm} / \mathrm{sec}$. In contrast, the voltage range of the analog-to-digital converters in the MTS control and data acquisition system is $\pm 10 \mathrm{~V}$. Because the system is linear, $+10 \mathrm{~V}$ is equivalent to $300 \mathrm{~cm} / \mathrm{sec}$ and $-10 \mathrm{~V}$ is equivalent to $-500 \mathrm{~cm} / \mathrm{sec}$, as illustrated in Figure 43. 


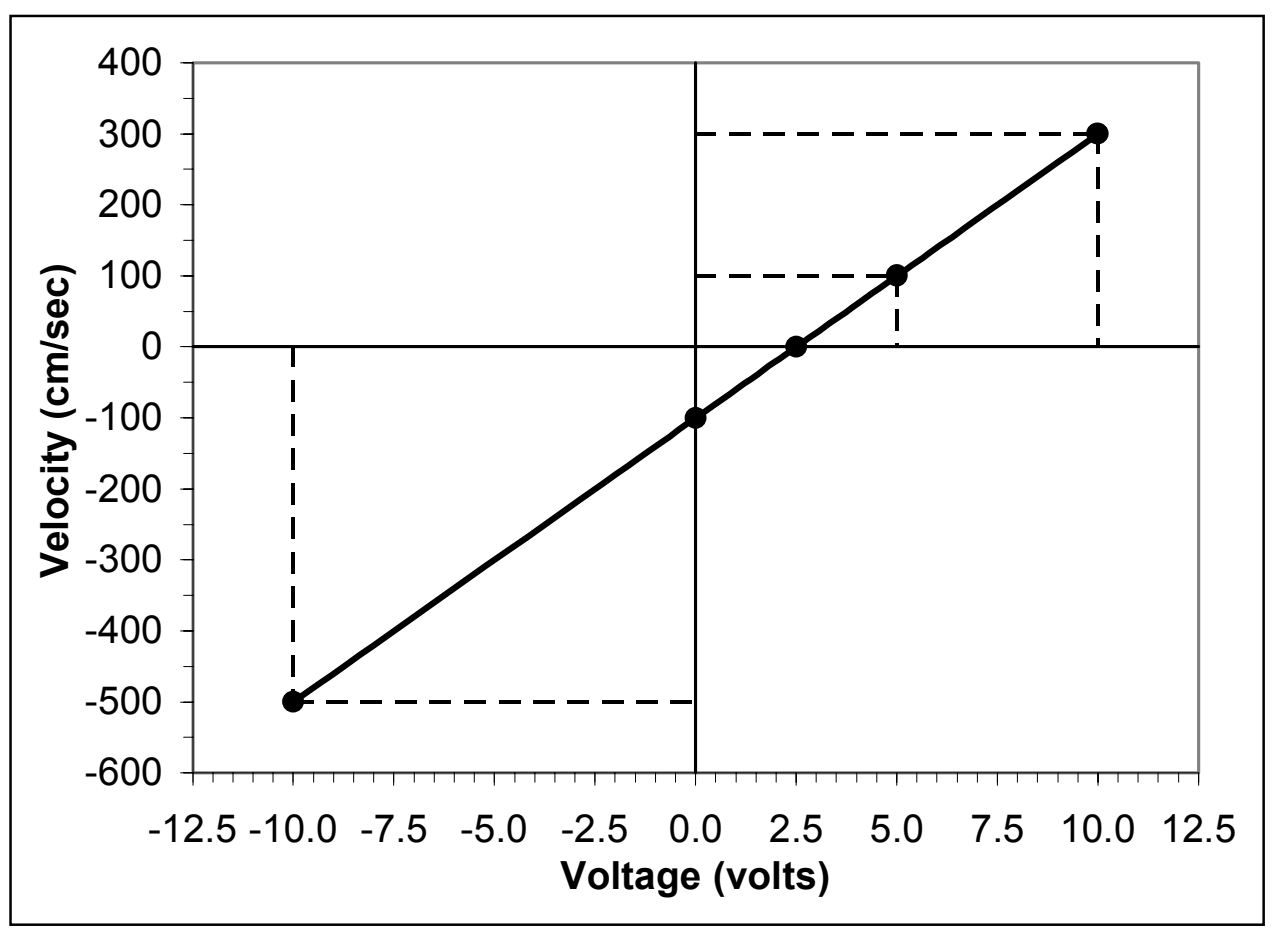

Figure 43. Relationship between the output voltage range from the ADVs and the range of the $A / D$ converters with respect to engineering units

Scaling the analog output signals from voltage to velocity is accomplished by using the Existing Sensors panel, shown in Figure 44, which is located under the Calibration menu of the MTS control software. The 38 ADV channels require scaling as shown in this manner.

Additionally, it is possible for the voltage level measured with the MTS control and data acquisition system to differ slightly than the voltage output from the ADVs. These differences are most likely caused by temperature fluctuations that cause variability in the impedance of the 130-m long analog cables. Therefore, the analog output signals from the ADVs are calibrated on a regular basis to compensate for these small variations. Calibration is performed by commands in the ADV software that force each ADV system to output a precise and constant analog voltage of either 0.000 or $5.000 \mathrm{~V}$. The water in the facility does not require motionless to conduct this procedure. The first step in the process is to force a given

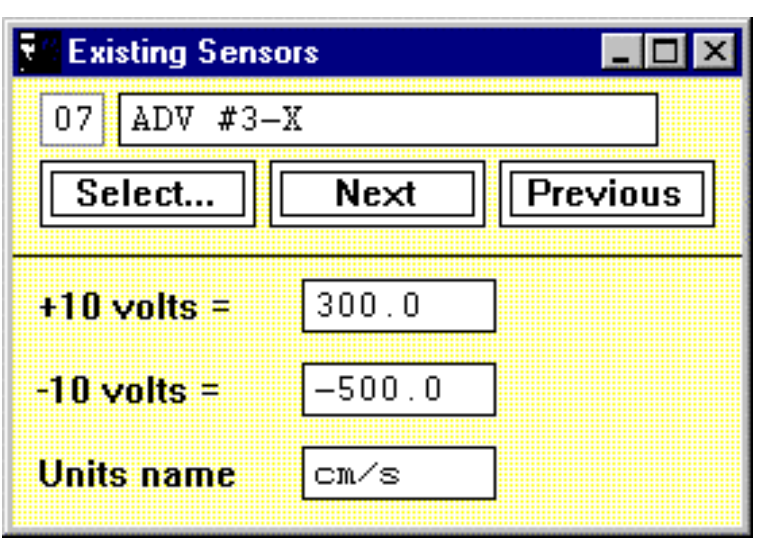

Figure 44. Existing sensors panel in the MTS control software is used to scale the analog output signals from voltage into engineering units (Permission to reprint granted by MTS Systems Corp.) ADV system to output $0.000 \mathrm{~V}$. If the analog-to-digital converter does not read exactly 0.000 volts, the value of the offset is adjusted using the $A / D-D / A$ Converters panel as shown in Figure 45. 
Next, the ADV system is forced to output $5.000 \mathrm{~V}$, and, the value of the gain is adjusted if the analog-to-digital converter does not read exactly $5.000 \mathrm{~V}$. This procedure is repeated for the $38 \mathrm{ADV}$ channels. This calibration process requires approximately $1 \mathrm{hr}$ and should be checked on a monthly basis to compensate for significant changes in ambient temperature.

\section{Measurement of currents}

The ADVs can be accurately positioned at any $x-y$ location within the testing region of the facility, as is the case for the wave gauges. The procedure used to measure currents is essentially the same as that used to measure waves. In

Figure 40 , the ADVs are evenly distributed every $1.5 \mathrm{~m}$ along the bridge, so that 10 ADVs cover a total range of $13.5 \mathrm{~m}$ in the cross-shore direction. The longshore position of the ADVs is selected from the control room by moving the instrumentation bridge to the desired transect location.

The sampling frequency and duration for measuring the currents is always identical to that used to measure waves because one of the objectives is to synchronize the wave and current measurements. In addition, the 38 ADV analog signals are recorded using the same Data Recorder panel in the MTS control software as the wave gauges, shown in Figure 42. Therefore, the sampling frequency and duration are the same for both wave and current measurements.

The orientation of the ADVs is critical. However, because of the highquality mounting devices that were built to mount the ADVs to the bridge, this issue only needs consideration when one or more of the ADVs are repositioned along the bridge. Upon initial installation of the ADVs, the y-axis was accurately aligned to be parallel with the longitudinal axis of the bridge. When an ADV is relocated along the bridge, the alignment of the vertical mounting struts, and hence, the orientation of the ADV is verified. The mounting mechanism ensures that the ADV does not rotate with respect to the vertical strut. The orientation of each ADV can be verified during a tow-tank experiment. If the ADVs have the proper orientation, the cross-shore velocities should be essentially zero as the bridge traverses along the support rails.

\section{Seeding material and SNR}

To ensure that high-quality velocity data are collected using the ADVs, a minimum SNR of 10 to 15 decibels $(\mathrm{db})$ is to be maintained throughout the sampling duration. The SNR is monitored using the real-time display system for the ADV digital data, shown in Figure 38. The ADV velocity data appears 
"noisy" when scattering material in the water is low. Low SNR was a problem during the fixed-bed hydrodynamic experiments, as discussed in Chapter 9. To solve this problem, the manufacturer recommended inserting into the water particles having a density near that of water and a mean diameter of 10 to 20 microns. Neutrally buoyant particles remain in suspension without additional stirring, and the size recommended provides a relatively strong echo per unit of concentration. The manufacturer recommended using hollow glass spheres with a mean diameter of about 10 to 20 microns. However, this special seeding material could only be purchased in large quantities at a cost of $\$ 200$ for $20 \mathrm{~kg}$.

After much trial and error, it was determined that the natural soil which exists in the Vicksburg, MS, area is an excellent soil to use as a seeding material. Therefore, prior to each fixed-bed hydrodynamic experiment, two $20-\ell$ buckets of dry soil were distributed throughout the testing region of the facility.

Immediately prior to the experiment, a hand-held rake was dragged through the water to stir the soil up into the water column. This increased the SNR readings from as low as $5 \mathrm{db}$ up to 15 to $25 \mathrm{db}$ even before waves were generated. After waves were generated, the SNR reached values ranging from 30 to $50 \mathrm{db}$, which is more than adequate to ensure high quality velocity data.

It should be noted that for the moveable-bed LST experiments, the sand used to construct the beach provides sufficient scattering material while waves are generated to produce SNRs of 30 to $40 \mathrm{db}$, and therefore natural soil does not need to be added as a seeding material.

\section{Quality of current data}

The reliability of velocity data obtained using acoustic current meters is adversely affected by the presence of air bubbles in the flow. The speed of a sound wave is changed by the presence of bubbles, resulting in a significant error in the velocity measurement, Hughes (1993). This is a problem encountered in the LSTF when making velocity measurements in very shallow water, since breaking waves force air bubbles down into the water column, sometimes to the depth of the ADV sensor. If this occurs, erroneous spikes appear in the velocity time series. However, these spikes are removed during postprocessing using a GEDAP filtering routine, developed specifically to remove spikes with these characteristics. Waves that break farther offshore do not cause a problem, because the air bubbles do not generally penetrate down to the depth of the acoustic transmitter and receivers.

The manufacture's performance specifications for the ADVs indicate an accuracy of \pm 1 percent of the measured velocity, or $\pm 0.0025 \mathrm{~m} / \mathrm{sec}$ for velocities less than $0.25 \mathrm{~m} / \mathrm{sec}$. However, it is very difficult to verify these specifications in a facility that holds $1,350 \mathrm{~m}^{3}$ of water, because the water rarely is totally motionless. Nonetheless, tow tank tests have been carefully conducted using the bridge and to tow all $10 \mathrm{ADV}$ s through the water at an average speed of 0.2 and $0.3 \mathrm{~m} / \mathrm{sec}$. A stopwatch was used to measure the elapsed time as the bridge moved at a constant speed over a distance of $20 \mathrm{~m}$. These tests were conducted for both the $\mathrm{x}$ - and y-axes of the ADVs and also were used to verify that the 
orientation of the ADVs was accurately aligned with the coordinate system used in the facility.

The average velocities obtained from the eight 3-D down-looking probes (ADVs 3 through 10) seemed to indicate that the performance specifications listed above were reasonable. However, the two 2-D side-looking probes (ADVs 1 and 2) measured significantly lower values on the y-axis than on the x-axis. For the 2-D side-looking ADVs, the positive $\mathrm{x}$-axis is defined from the acoustic transmitter to Acoustic Receiver No. 1 (painted red). The positive y-axis is defined along the axis perpendicular to the acoustic transmitter from the sensor towards the sampling volume. After much investigation, it was determined that the acoustic transmitter and receivers on the side-looking sensors cause significant flow disturbance when water is flowing parallel to the y-axis of the ADV. Because quantifying the longshore current in the facility is a higher priority than quantifying the cross-shore current, the decision was made to permanently rotate ADVs 1 and 2 by 90 deg in the clockwise direction. Therefore, the positive $\mathrm{x}$-axes of ADVs 1 and 2 are aligned to be parallel with the negative $y$-axis of the coordinate system used in the facility (i.e., downstream). In addition, the analog voltage cables for ADVs 1 and 2 were reversed on the back of the MTS data acquisition system so that the orientation of the ADV axis remains consistent with the overall coordinate system used in the facility. In addition, the voltage signal signs were transposed so that the positive and negative directions were correct. This was accomplished by changing the appropriate signs on the $\mathrm{x}$ - and y-axes of ADVs 1 and 2, using the Existing Sensors panel showed in Figure 44. Additional information in regard to the operation of the ADV software and hardware can be found in the Operation and Reference Manual, SonTek (1997).

\section{Analysis of currents}

A GEDAP batch file was developed to expedite the procedure of postprocessing several transects of current data. The general procedure consists of the following steps. First, the program GLITCH_FIX_4A is used to remove spikes in the velocity time series caused by air bubbles entrained in the water column. This program was developed specifically for the type of spikes observed in the output time series from the ADVs used in the LSTF. Input parameters required to operate the program are: (a) the time scale length (TSL) of the sliding window that moves along the time series; and (b) the number of standard deviations, Alpha, used to define the upper and lower limits of the valid data with respect to the mean value within the sliding window. Initially, the program searches the input record to find the TSL segment having the smallest standard deviation. The purpose of this search is to provide an initial estimate of the mean and standard deviation of the true signal without noise glitches. A TSL value of $5.0 \mathrm{sec}$ worked well for experiments conducted with a peak wave period of $2.5 \mathrm{sec}$.

Input parameter Alpha is usually set to a value between 3.0 and 4.0. Theoretically, a value of Alpha $=3.5$ should have no measureable influence on the true signal, assuming that the data have a Gaussian distribution. Based on experience, Alpha is typically selected as 3.5 , which sets the initial data limits to 
be \pm 3.5 times the standard deviation about the mean. Data points outside the data limits are discarded, and the mean value is recalculated for the remaining data set within the sliding window. Smaller values of Alpha sometimes are used for ADVs 1 and 2 because these signals contain many glitches that result from the presence of air bubbles in shallow water. However, good judgement is required in the selection of Alpha, because valid parts of the signal may be removed. The remaining set of valid data points is sorted into increasing order by time, and linear interpolation is used to replace points removed by the program. Therefore, the length of the filtered time series is the same as the original time series, and the 38 channels of current data remain synchronized. Normally, only ADVs 1 through 4 need to have glitches removed from the velocity signal, since these are the sensors that are located in shallow water depths.

At this step in the analysis, the batch file pauses allowing the user to superimpose the filtered time signal onto the original time series, which contains spikes caused from the entrained air bubbles. This is integrated into the GEDAP batch routine and is plotted using GPLOT, as shown in Figure 46. This figure shows the results of filtering the y-axis (longshore) time series for ADV 2. These results were obtained using a value of Alpha $=3.5$, which works well if the true signal has a Gaussian distribution. It sometimes requires several iterations to determine the optimum value of Alpha. However, it should be mentioned that in some cases, the number of spikes are so numerous that the signal cannot be properly filtered to obtain a time series free of spikes. If this is the case, the time series is discarded. A more complete description of this filtering routine is available in the GEDAP on-line documentation.

After the signals in shallow water have been filtered, the program STAT1 is used to calculate the minimum, mean, maximum, and standard deviation of the velocity signals for both the $\mathrm{x}$ - and $\mathrm{y}$-axes. The mean value obtained from the $\mathrm{y}$ axis of the $10 \mathrm{ADVs}$ defines the cross-shore distribution of the longshore current.

Finally, the GEDAP program PLUCK is used to collect the values of the four statistical parameters calculated using STAT1 from the header of each of the output files. The final result is an ASCII file consisting of six columns of data. The first two columns identify the channel number and ADV number and axis. The last four columns contain the values of the four statistical parameters discussed above for all 38 ADV channels.

As mentioned previously, as many as 12 transects have been measured during a $2 \mathrm{hr}$ segment of an experiment. In contrast to the GEDAP batch file used for postprocessing the wave data, the batch file processes only one transect of current data, because several iterations may be necessary to determine an optimum value of Alpha. One transect of current data can be analyzed in approximately $15 \mathrm{~min}$. Therefore, approximately $3 \mathrm{hr}$ would be usually be required to process 12 transects of current data.

Examples of the cross-shore distribution of the measured mean longshore current during a regular wave and irregular wave experiment are shown in Figures 66 and 68, Chapter 9. 


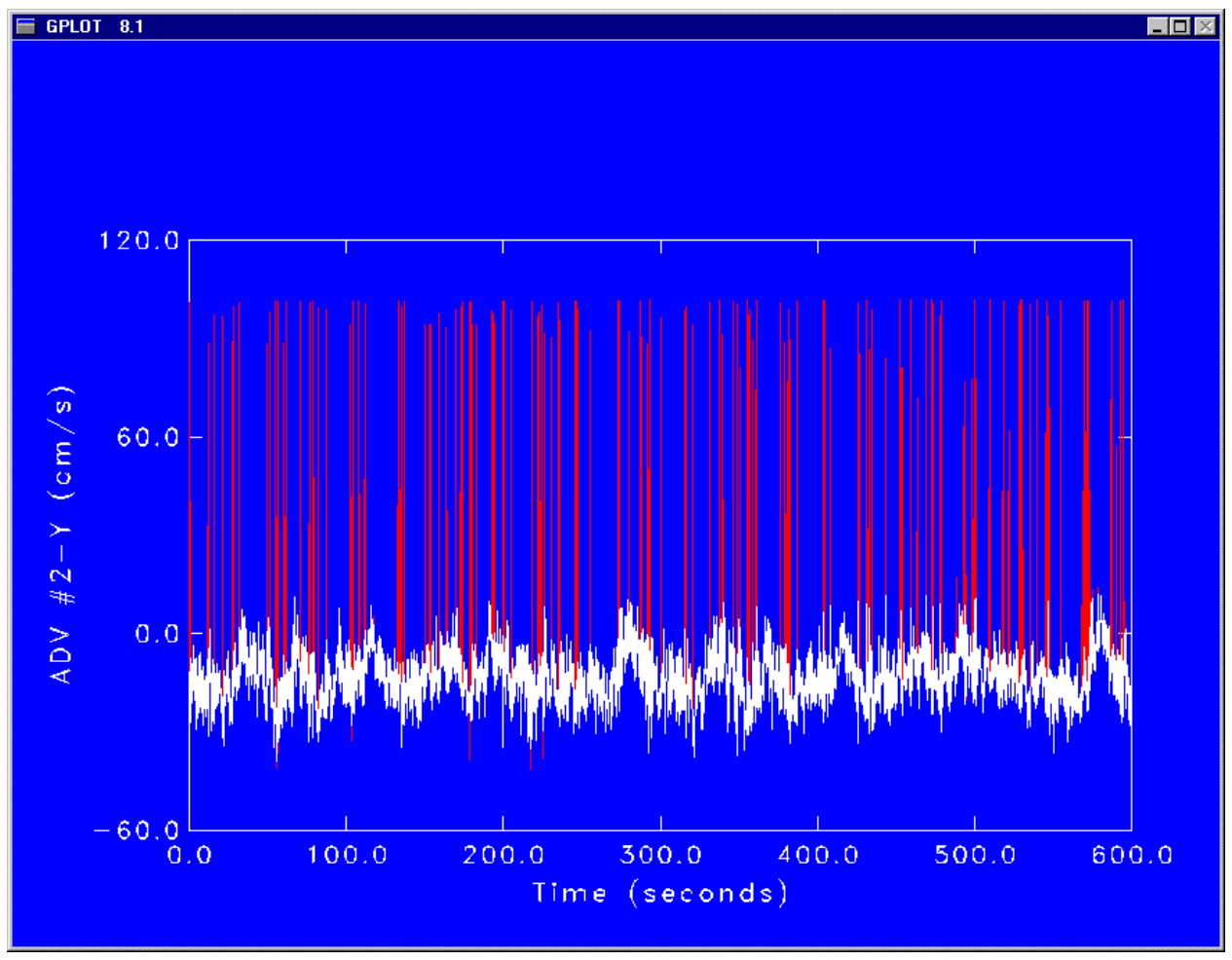

Figure 46. Comparison of longshore current measured with ADV No. 2, before and after removing spikes caused by air bubbles in shallow water

\section{Sediment Concentration Data}

As mentioned previously in Chapter 2, four vertical arrays of Fiber-Optic Backscatter Sensors (FOBS) are located along the length of the bridge. Each array of FOBS simultaneously measures sediment concentration at 19 elevations above the sand bed. Each vertical array consists of a lower and an upper array. The lower array is designed to measure sediment concentration close to the bed, where the gradients in concentration are largest. This array has eight sensors with a vertical spacing of $1.0 \mathrm{~cm}$ and has sufficient mechanical strength to penetrate slightly into the sand bed without being damaged. The bottom sensor is deployed directly on the bottom to serve as an elevation reference for the remaining sensors. The upper array has 11 sensors with a nearly logarithmic vertical spacing and measures the sediment concentration in the upper portion of the water column where the gradients are generally weaker. Each vertical array, consisting of 19 sensors, covers a total vertical range of $0.53 \mathrm{~m}$. Therefore, when located in shallow water, the upper array may be located above the water surface.

\section{Calibration of FOBS}

Each of the four vertical arrays was calibrated in a calibration tank, consisting of a sealed vertical chamber which holds a water-sand mixture and has a known volume. A pump recirculates the water-sand mixture through the 
vertical chamber, maintaining a homogeneous concentration of sand within the vertical chamber. The 19 sensors on a vertical array are calibrated simultaneously, and each sensor is sampled at a frequency of $16 \mathrm{~Hz}$ for a duration of $120 \mathrm{sec}$.

The first data point is obtained with no sand in the water. A second data point is obtained after an accurately measured mass of sand is added to the mixing chamber to obtain a sediment concentration of $10 \mathrm{~g} /$ liter. This process is repeated for sediment concentrations ranging from 0 to $80 \mathrm{~g} /$ liter, in increments of $10 \mathrm{~g} /$ liter. The FOBS were calibrated using the same sand that was used to construct the moveable-bed beach in the LSTF.

Figure 47 shows the calibration results for Sensor No. 1 (i.e., the lowest sensor) on Vertical Array No. 1. The manufacturer of the FOBS has found that for clean beach sand, the magnitude of the output signal from the sensors (measured in "counts") is a nearly linear analog of suspended sediment concentration, over the range from 0 to $100 \mathrm{~g} /$ liter. However, it was found that for the sand used in the LSTF $\left(\mathrm{D}_{50}=0.15 \mathrm{~mm}\right.$, with a narrow particle size distribution), a third-order polynomial is needed to match the low and high range of the calibration data. In addition, the scale used to represent the magnitude of the output signal is quite large (i.e., counts ranging from approximately 2,000 to 4,000), and more significant digits are needed to define the coefficients of the polynomial with sufficient accuracy. Based on the results from a sensitivity analysis, seven significant digits are used for each coefficient in the equation, although only five are shown in the figure for clarity.

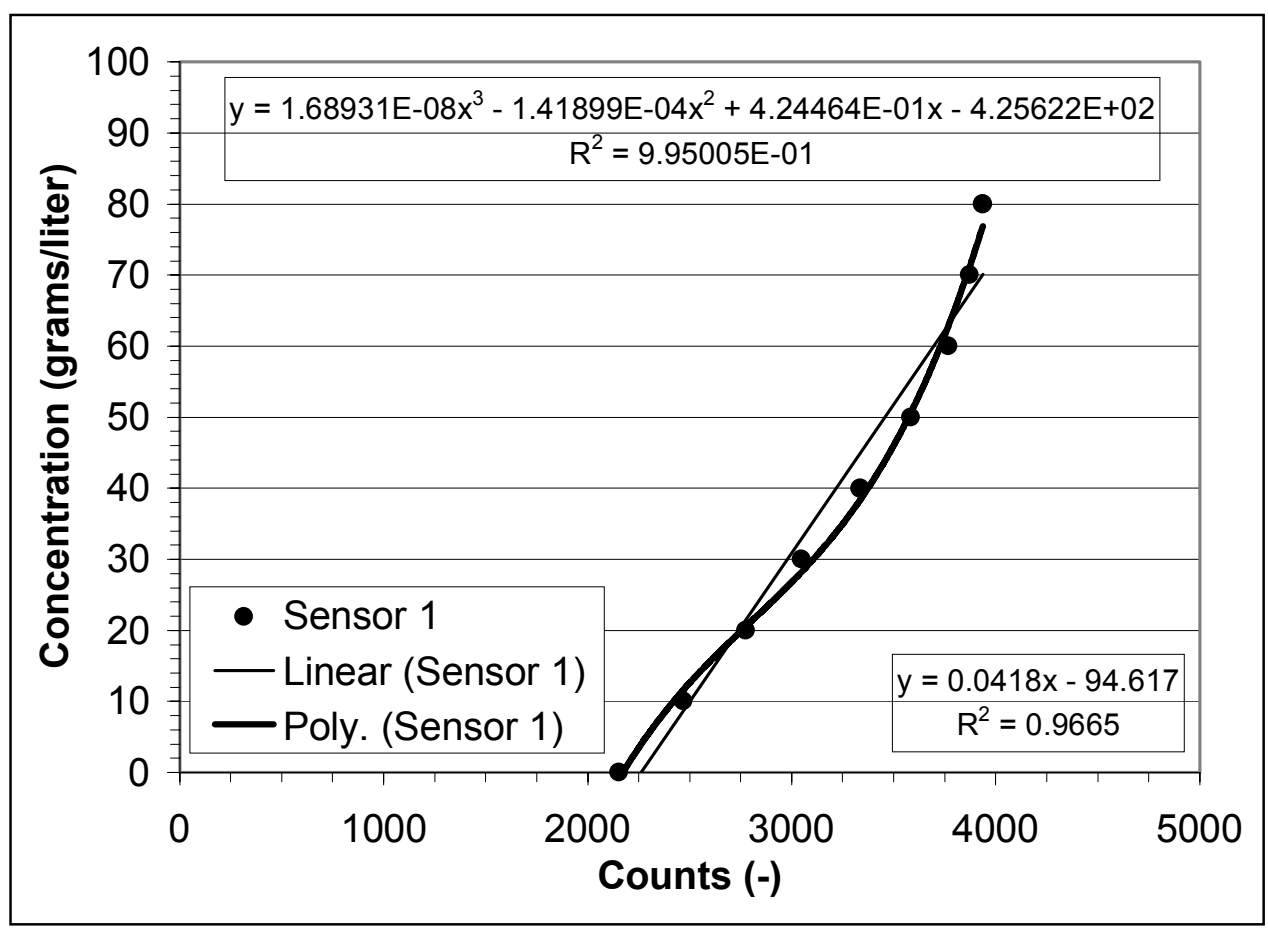

Figure 47. Calibration results for FOBS No. 1 on Vertical Array No. 1 


\section{Measurement of sediment concentration}

The FOBS can be relocated at various positions along the length of the bridge in a similar manner in which the wave gauges and current meters are distributed. Each array of FOBS is co-located about midway between the wave gauges and the current meters. Figure 40 shows, one of the vertical arrays midway between the wave gauge and the current meter, directly beneath the person standing on the bridge. In this particular case, a large portion of the upper array was above the water surface, since the water depth was significantly less than the vertical range of the 19 sensors on the vertical array.

One of the most common problems associated with sediment concentration measurements is determining the elevation of the sensors relative to the surface of the sand bed. A standard procedure was developed to solve this problem. The procedure requires two people, one to monitor the FOBS data acquisition computer and the other to adjust the elevation of the vertical array of FOBS. The vertical array is positioned so that the lowest sensor is well above the elevation of the sand bed. The vertical array is slowly and carefully lowered until the output signal from the lowest sensor gives a full-scale reading, which indicates that the lowest sensor has penetrated the surface of the bed. If the vertical array is lowered too far, the second lowest sensor will penetrate the sand and also will output a full-scale reading. If no waves are present, the array is raised until the output signal from the second lowest sensor returns to zero. However, if waves are present and sediment is present in the water column, the array must be redeployed. After the array is positioned correctly, the elevation of the vertical array is locked into position, and this procedure is repeated for the remaining vertical arrays. At this point, data collection can begin.

Using this method, the maximum vertical positioning error is $\pm 1.0 \mathrm{~cm}$. However, with experience, it was determined that the elevation of the lowest sensor can be positioned with a vertical accuracy of $\pm 0.5 \mathrm{~cm}$ relative to the surface of the bed. However, ripples are always present and are constantly moving; therefore, the vertical positioning accuracy will decrease somewhat. In general, this method works well while waves are generated, even if the person lowering the vertical array cannot see the lowest sensor or the elevation of the sand bed, which is usually the case. Prior to moving the bridge to the next transect, the four vertical arrays of FOBS are raised so that the lowest sensors are not touching the surface of the sand bed.

During an experiment, the four arrays of FOBS are measured simultaneously (i.e., a total of 76 sensors) at a frequency of $16 \mathrm{~Hz}$ to provide sufficient temporal resolution to measure sediment resuspension events, which often occur over small fractions of a wave period. The sampling duration is equal to the duration of the wave machine drive signal, so that the average sediment concentration obtained from the measurement is an accurate representation of the average concentration generated by the sequence of irregular waves in the wave machine drive signal. Therefore, the sampling duration of the FOBS is always the same as the sampling duration for the wave and current data. 


\section{Analysis of sediment concentration}

The first step in processing FOBS data is to remove all of the data points with a count value less than that obtained during the calibration procedure with no sand in the water (i.e., the $\mathrm{x}$-intercept in Figure 47). This is based on the assumption that sediment concentration should not be less than clear water with zero concentration. These low values occur if the water surface drops below the elevation of a sensor, which occurs frequently for the upper sensors located in shallow water.

The second step is to calculate the average count value for the remaining time series. This average count value is used as input into the appropriate calibration equation, and a sediment concentration is obtained.

The last step is to calculate the average concentration during the entire sampling duration, which can be much lower than the average concentration obtained from the time series after the unrealistically low values have been removed. Essentially, this step replaces the unrealistically low values with zero counts (representing clear water) and is averaged over the sampling duration.

To illustrate the steps involved in processing the data, Figure 48 shows the same vertical concentration profile at the three different stages of postprocessing. These measurements were obtained using Vertical Array No.1 in relatively shallow water during an LST experiment. The first data set represents the average concentration obtained using the time series prior to any postprocessing. The second data set was obtained after the unrealistically low values in the original time series had been removed, resulting in a significant increase in the average concentrations measured in the upper water column. The third data set represents the average concentrations during the entire sampling duration, resulting in a significant decrease in the average concentrations measured in the upper water column. This figure also shows that the concentrations measured lower in the water column remain unchanged, because these original time series did not contain any unrealistically low count values.

\section{Bathymetric Data}

Conducting a high resolution, bathymetric survey of a moveable-bed beach with cross-shore dimensions of $18 \mathrm{~m}$ and longshore dimensions of $30 \mathrm{~m}$ could be very laborious and time-consuming. Therefore, as discussed previously in Chapter 2, a fully automated, 3-D, bathymetric survey system was developed to quantify spatial and temporal changes in the moveable-bed beach. Since the facility holds a large volume of water, it is not practical to drain the facility every time bathymetric measurements are needed. Therefore, an amphibious profile indicator is used so that changes above and below the still-water level could be measured. The profile indicator is shown in Figure 8. 


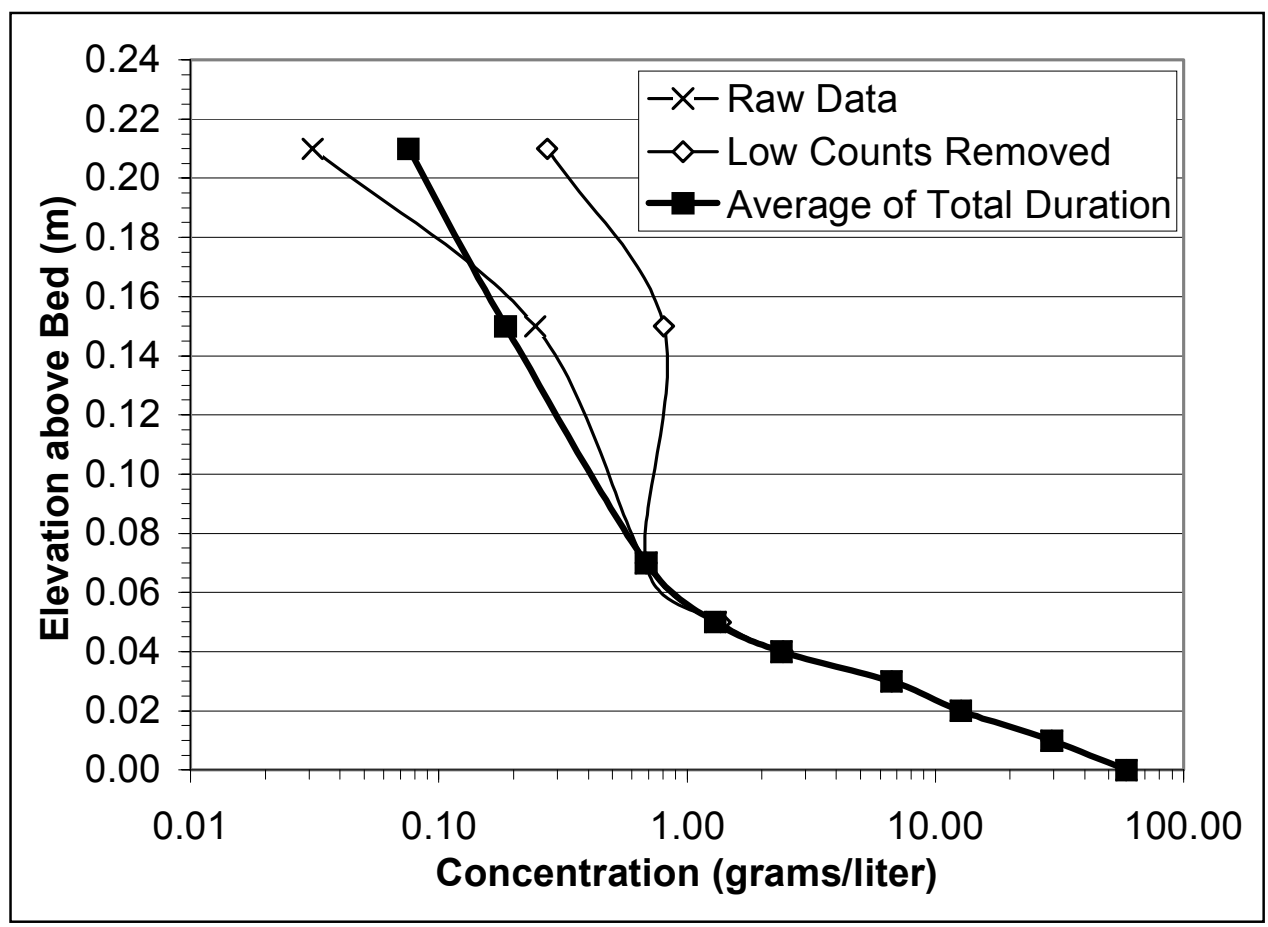

Figure 48. Calculation of a vertical sediment concentration profile

\section{Calibration of beach profile indicator}

A permanent benchmark was installed to verify the "offset" in the calibration equation for the profile indicator, to ensure consistency from one experiment to the next. The benchmark is located at $X=0.697 \mathrm{~m}$ and $\mathrm{Y}=16.015 \mathrm{~m}$ on top of the concrete beach and can be accessed by lowering the sensor down through the moveable-bed into a 0.2-m-diam polyvinyl chloride pipe, as shown in Figure 49. The surface of the benchmark is located at an elevation of $Z=0.742 \mathrm{~m}$. A graded scale, with a $1.52-\mathrm{m}$ vertical range, is located on the front of the vertical shaft of the profile indicator. The elevation of the vertical scale was adjusted so that the vertical scale reads exactly $0.742 \mathrm{~m}$ if the sensor is resting on the benchmark. The scale has a gradation of $0.01-\mathrm{m}$ major increments and $0.001-\mathrm{m}$ minor increments.

The profile indicator is calibrated using a similar procedure as that used to calibrate the linear actuators that control the elevation of the wave gauges. The $P V-12$ Profiler Calibration panel is accessed under the Calibration menu of the MTS control software, (Figure 50). The first step in the calibration process is to lower the sensor onto the benchmark. After the sensor is motionless, the "Read Cal Step," or toggle, button is pressed and the MTS control software records the output voltage from the profile indicator. The operator manually enters the physical elevation of the sensor in the "Measured Value" column. For example, in Figure 50, the first calibration step obtained with the sensor resting on the benchmark (measured elevation of $0.742 \mathrm{~m}$ ) has an output voltage of 6.014 volts. The profile indicator is moved offshore to a location where the sensor can be lowered down close to the horizontal floor of the facility. This is approximately 


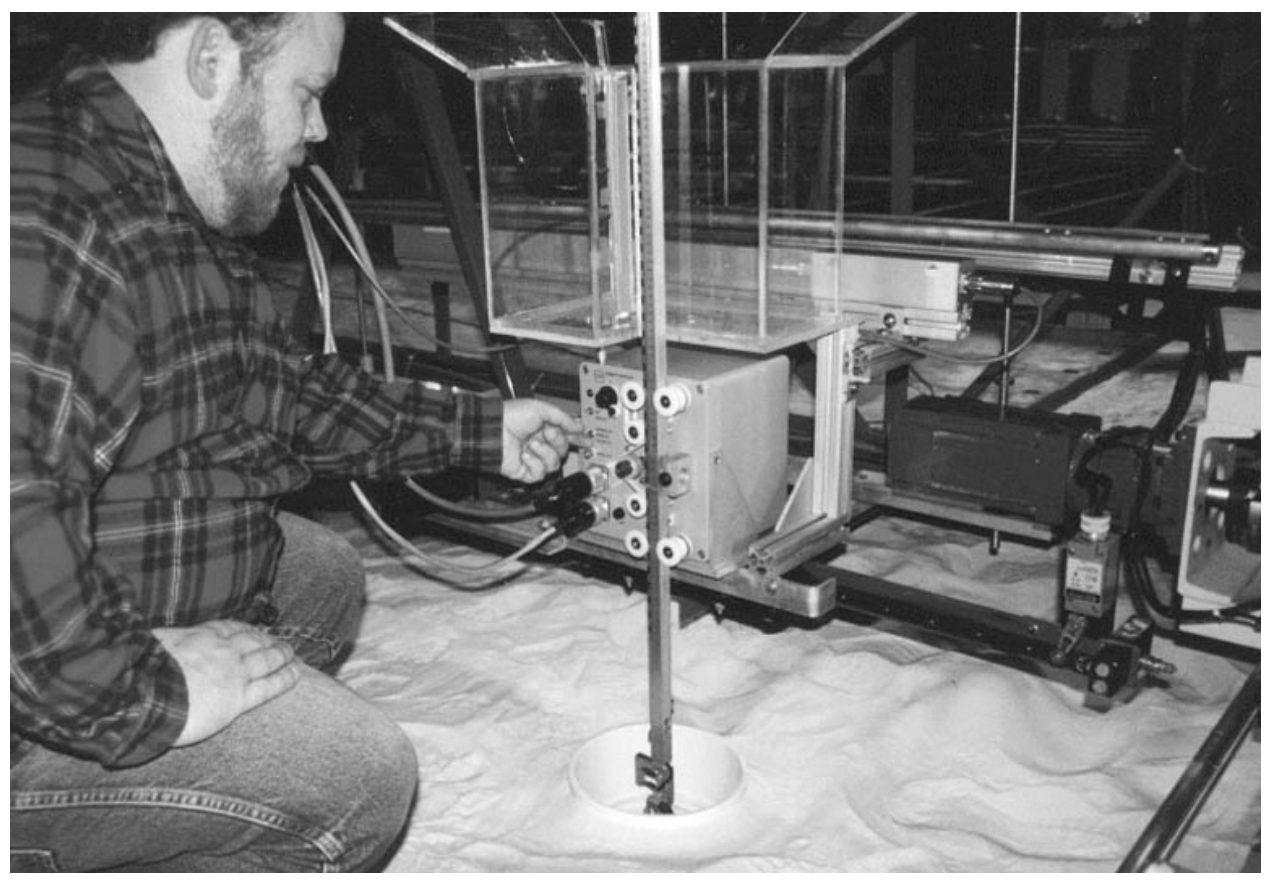

Figure 49. Calibration of beach profiler indicator is verified on benchmark

the lowest limit that the profile indicator can reach, and is lower than the lowest elevation on either the fixed-bed or the moveable-bed beach. After the sensor is lowered near the horizontal floor and the sensor is motionless, the toggle button is pressed and the second output voltage is recorded. The operator manually enters the physical elevation of the sensor. The vertical shaft of the profiler is raised $0.1 \mathrm{~m}$, and the third calibration point is recorded. This process is repeated in increments of $0.1 \mathrm{~m}$ using the vertical scale to obtain the physical elevation at each calibration step. After all 15 data points have been collected; the toggle button is pressed to calculate the best-fit linear equation through the data points, and a linear equation is used represent the measured calibration data. This completes the calibration process. Additional information on the beach profile indicator can be obtained in Delft Hydraulics (1998).

To ensure consistency from one experiment to the next, and that the sensor has not "drifted" since the previous survey, the calibration of the profile indicator is verified prior to and immediately after each bathymetric survey. Experience has shown that the profile indicator may drift electronically by as much as $\pm 2 \mathrm{~mm}$ between two consecutive surveys, generally representing a time period of 24 to $72 \mathrm{hr}$. This drift offset is removed numerically when the wheel of the profile indicator is resting on the benchmark. The Converters panel in the MTS control software is opened and the analog channel associated with the profile indicator is selected in a similar manner as was shown for the ADVs in Figure 45. The value of the offset is adjusted slightly until the voltage output with the wheel on the benchmark is identical to the voltage output obtained during the calibration process with the wheel on the benchmark. After this is completed, the user can begin conducting the bathymetric survey. 


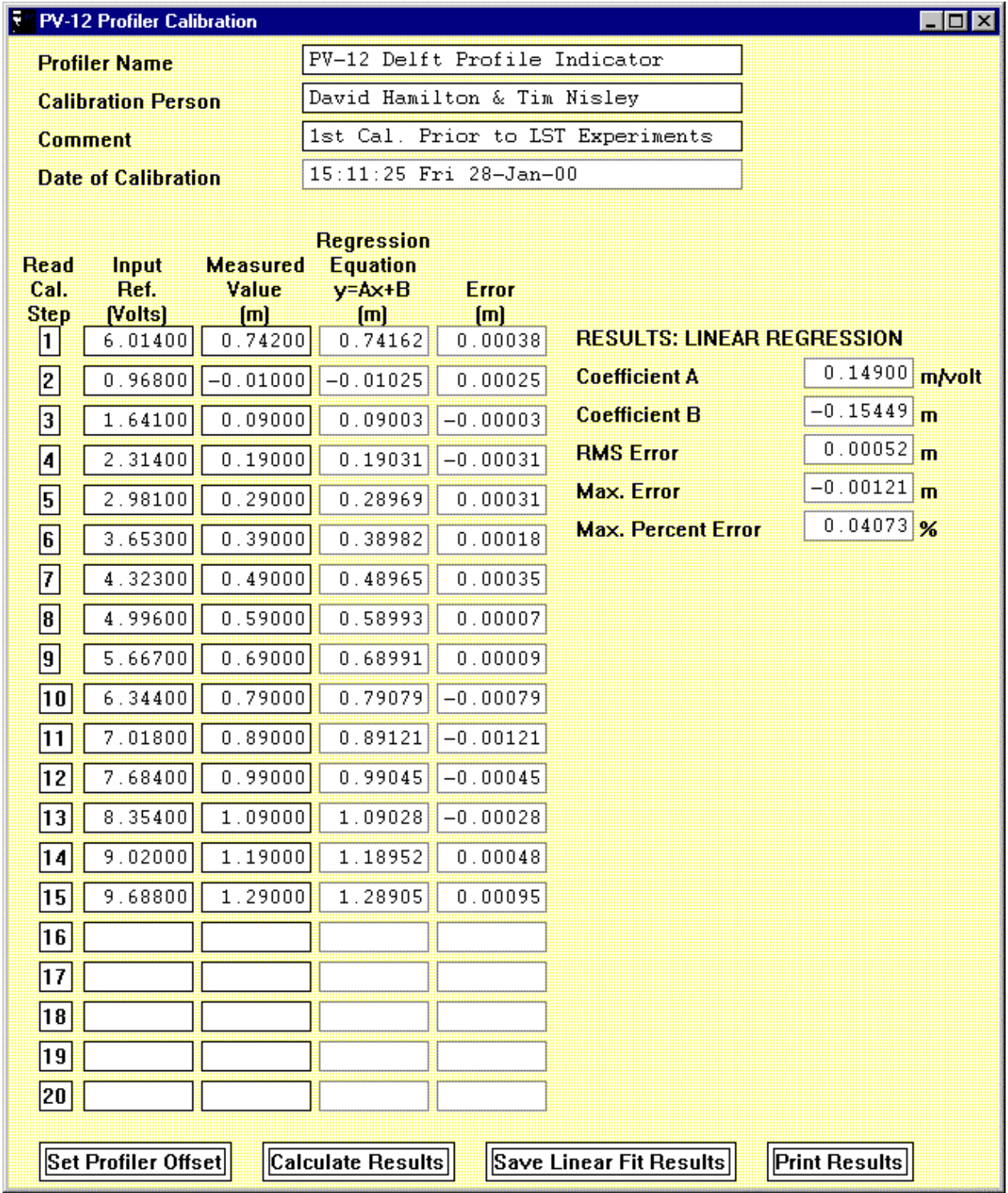

Figure 50. Calibration panel in the MTS control software for the beach profile indicator (Permission to reprint granted by MTS Systems Corp.)

\section{Measurement of beach profiles}

The PV-12 Profiler Operation panel, shown in Figure 51, can be accessed under the Operation menu of the MTS control software. This panel is used to define the longshore location, as well as the onshore and offshore ends of each transect. For the example shown in this figure, 39 transects were measured, one transect every $0.5 \mathrm{~m}$ along the beach. The system is fully automated and operates as follows. First, the MTS control software moves the support platform to locate the sensor at the offshore $\mathrm{x}-\mathrm{y}$ position of the first transect. The system lowers the sensor to the moveable-bed. Next, the $\mathrm{x}$-axis drive motor moves the profile indicator in the shoreward direction at the selected speed of $0.1 \mathrm{~m} / \mathrm{sec}$. The $\mathrm{x}, \mathrm{y}$, and $\mathrm{z}$ positions of the sensor are measured every $0.005 \mathrm{~m}$ in the cross-shore 


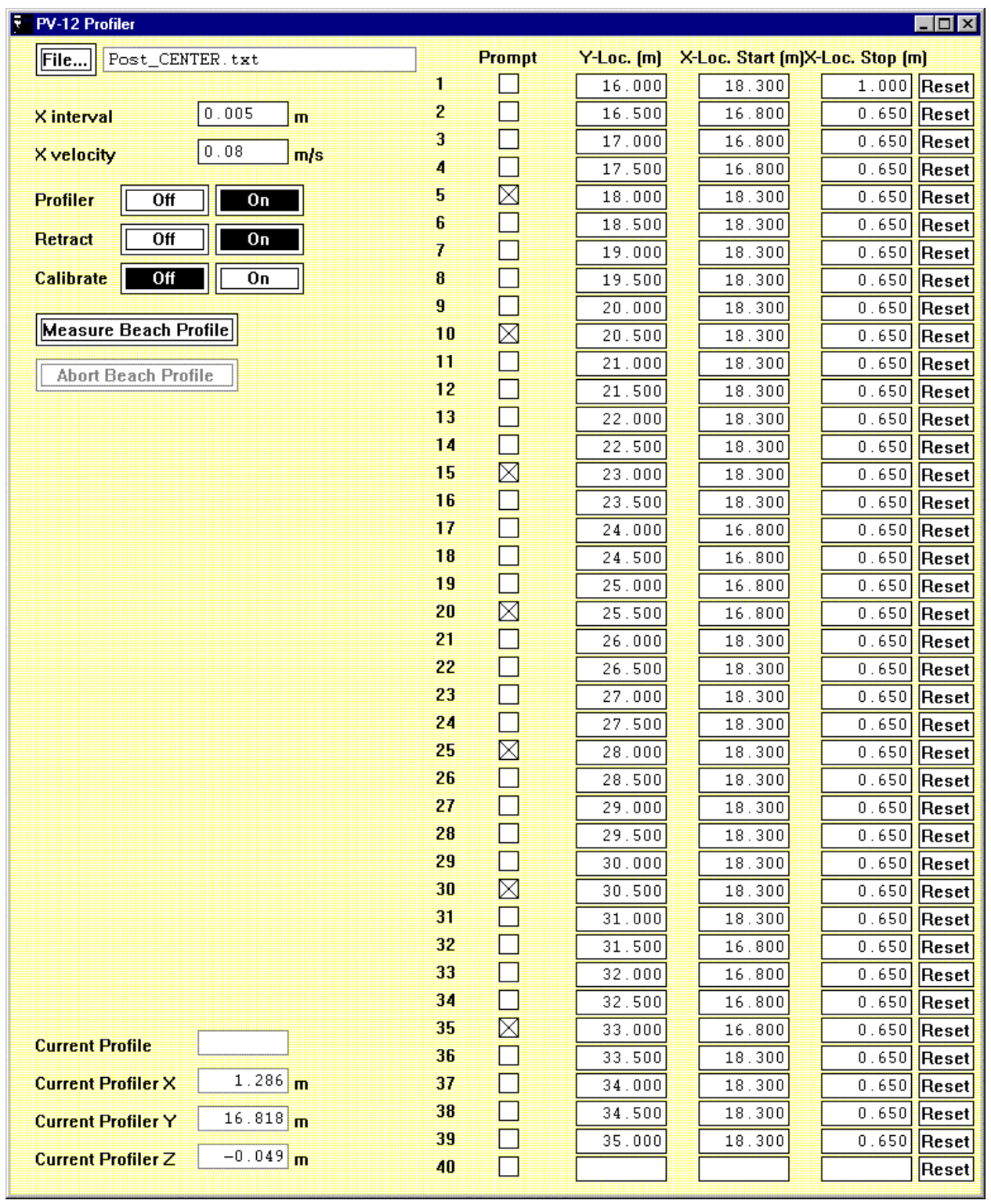

Figure 51. Operation panel in the MTS control software for the fully automated bathymetric survey system (Permission to reprint granted by MTS Systems Corp.)

direction as the wheel rolls along the surface of the sand bed. The servo motor inside the profile indicator maintains a continuous downward force on the bed equivalent to a mass of $40 \mathrm{~g}$. After the sensor reaches the shoreward end of the transect, the system raises the sensor to the fully retracted position, which completes one transect. This process is repeated for each transect selected in the $P V-12$ Profiler Operation panel. During the survey process, the $\mathrm{x}, \mathrm{y}$, and $\mathrm{z}$ positions of the sensor are displayed real-time in the lower left corner of the $P V$ 12 Profiler Operation panel.

Because the moveable-bed beach is $30 \mathrm{~m}$ long, it requires approximately 60 transects to complete a bathymetric survey, with one transect every $0.5 \mathrm{~m}$ along the beach. Therefore, three $P V-12$ Profiler Operation panels are normally used 
for a complete bathymetric survey. The setup parameters for each panel are saved in three different "settings.set" files and are usually named "Setting downdrift.set," "Settings_middle.set," and "Settings_updrift.set." The updrift and downdrift configuration files contain approximately 10 transects in each.

Each transect requires approximately $5 \mathrm{~min}$ to complete; therefore, a total bathymetric survey consisting of 60 transects requires approximately $5 \mathrm{hr}$ to complete. The operator only needs to be involved in this process at the beginning and end of each of the three segments of the survey.

The manufacturer's performance specifications indicate a vertical sensitivity of $\pm 0.2 \mathrm{~mm}$ and a linearity of 0.1 percent of full scale. Although these are excellent performance characteristics, only the resolution of the potentiometer is considered, rather than the entire system. Nonetheless, during preliminary performance tests conducted with a rigid piece of metal positioned along one of the profile transects, the profile indicator measured the elevation of the rigid metal surface within $\pm 1 \mathrm{~mm}$ of the actual elevation after 10 repetitions.

Therefore, the accuracy and repeatability of the sensor has proven to be excellent and meets the requirements of the facility.

\section{Analysis of beach profiles}

The raw output files containing the measured beach profile data are in ASCII file format and consist of three columns of data, the $\mathrm{x}, \mathrm{y}$, and $\mathrm{z}$ values. The profile depth is measured every $0.005 \mathrm{~m}$ in the cross-shore direction. The $\mathrm{x}, \mathrm{y}$, and $\mathrm{z}$ positions are recorded with an accuracy of $0.001,0.01$, and $0.0001 \mathrm{~m}$, respectively. Consecutive beach profiles are listed one below the other. The data are clean and need not be filtered or altered in any way.

This large amount of high-resolution bathymetric data can be analyzed using various qualitative and quantitative techniques. The standard analysis for each test segment consists of two types of qualitative analysis. First, a 2-D contour plot is generated using surface mapping software to visualize the uniformity of the bathymetry. These contour plots provide considerable qualitative information and are used, in part, to determine whether or not the magnitude of the erosion at the updrift end of the beach is sufficient to dictate dredging of the sediment traps. Secondly, a contour plot of the difference between the posttest and pretest bathymetry gives insight in regard to the degree of erosion and accretion in various regions of the beach.

Two types of quantitative analysis are also conducted after each test segment. First, each of the measured beach profiles from the posttest survey is superimposed onto the corresponding beach profiles from the pretest survey. These plots are used to quantify various changes in the beach profile, such as the location of the still water shoreline and the maximum depth of erosion or accretion. Secondly, the net volumetric change along each beach profile is calculated assuming that each profile is representative of a $0.5 \mathrm{~m}$ wide slice of beach which runs along the beach profile (i.e., $0.25 \mathrm{~m}$ on each side of the profile line). The net volumetric change within the spatial domain of each slice is plotted as a function of longshore position. This type of analysis is very useful in 
quantifying the longshore uniformity of the net volumetric change along the beach. More specifically, it also is helpful in quantifying the degree of erosion at the updrift end of the beach, as well as the rate of growth of the submerged shoal, which tends to form immediately updrift of the downdrift wave guide.

Examples of these types of analysis will be discussed in detail in Chapter 10 . These examples are based on the results from two sets of moveable-bed experiments, one with wave conditions characterized by spilling waves and the other for plunging waves.

\section{Sediment Trap Data}

The design of the sediment trapping system was discussed previously in Chapter 4. This section will discuss additional information related to calibration of the load cells, the methodology used to obtain an accurate measurement of the weight of sand in each trap, and the data analysis procedures used after a test segment is complete. As mentioned previously in Chapter 4, the term "submerged weight" will be used rather than "mass," because submerged weight the quantity measured by the traps.

\section{Calibration of load cells}

Each of the 60 load cells that support the 20 sediment traps consist of high accuracy, shear beams constructed inside of a stainless steel, air-tight, and splashproof enclosure. The manufacturer's performance specifications indicate a maximum combined error of \pm 0.05 percent of the rated capacity of the load cells, considering the combined effect of linearity, repeatability, and hysteresis. Therefore, the maximum combined error for the 250-, 500-, and 1,000-kg load cells is $0.125,0.25$ and $0.5 \mathrm{~kg}$, respectively. The linear calibration equations were verified in the electronics laboratory prior to installation, and the 60 load cells met the manufacturer's performance specifications. Therefore, the linear calibration equations supplied by the manufacturer were initially used.

These specifications, however, do not consider the effect of seasonal temperature fluctuations, which can vary by as much as $40{ }^{\circ} \mathrm{C}$ in the Vicksburg, MS, area. The manufacturer's specifications indicate a variability of \pm 0.007 percent of the rated capacity of each load cell, per degree centigrade change in ambient temperature. Assuming a $40-{ }^{\circ} \mathrm{C}$ change in ambient temperature, the temperature induced variability for the $250-, 500-$, and $1,000-\mathrm{kg}$ load cells is 0.7 , 1.4 , and $2.8 \mathrm{~kg}$, respectively. This temperature induced potential error is 5.6 times larger than the errors associated with linearity, repeatability, and hysteresis.

To minimize the errors induced by seasonal temperature fluctuations, and therefore improve the overall accuracy of the sediment trapping system, the data acquisition system was designed so that a "shunt cal" procedure can be performed on the 60 load cells at the beginning of each experiment. The procedure works by electronically applying a known resistance to the 60 load cells. This additional resistance increases the output voltage from each load cell by approximately one-half of the total voltage range (or rated capacity) of each 
load cell. This increase in output voltage (i.e., electronically induced increase in weight) was accurately measured in the electronics laboratory prior to installing the load cells in the sediment trapping system. During the "shunt cal" procedure (prior to an experiment) the "gain" of the linear calibration equation is adjusted so that the increase in output voltage exactly matches the increase in voltage that was measured prior to installation. In other words, the "gain" in the calibration equation is slightly adjusted to compensate for the effect of a change in temperature on the load cells, cabling, and other components. This procedure has been automated and can be conducted in approximately $10 \mathrm{~min}$ at the beginning of each experiment. From theoretical considerations alone, this procedure only needs to be performed when the ambient temperature has changed significantly since the last "shunt cal" was conducted. However, it is prudent to perform this procedure at the beginning of each experiment, because it provides a means of verifying that all 60 load cells are functioning properly.

Furthermore, the "shunt cal" procedure is only performed after the sediment traps have been dredged and the 20 traps are essentially empty of sand. This is necessary because the calibration file associated with the "shunt cal" procedure adjusts the offset of each calibration equation so that each trap weighs $0.0 \mathrm{~kg}$ after the procedure is complete. If the traps each contain a considerable amount of sand, it is necessary for the operator to input the most recently measured weight on each load cell (i.e., measured at the end of the last test segment). This process is somewhat tedious, because 60 values must be transferred from the most recently measured data file to the calibration file. Therefore, the standard procedure is to conduct a "shunt cal" only after the traps have been dredged.

\section{Measurement of sediment trap data}

The physical process of sand accumulating in the sediment traps is a relatively slow process. A typical test segment lasts from 1 to $3 \mathrm{hr}$ of continuous wave forcing and current recirculation. During the design process for the sediment traps, the following tentative assumptions were made. The first assumption is that during later stages of an experiment, after the beach profile has approached an equilibrium shape, the rate of accumulation of sand in a given trap is relatively constant (i.e., increase linearly) throughout a test segment. The second assumption is that during the early stages of an experiment, as the beach profile is adjusting more rapidly, the rate of accumulation in a given trap may not increase with a linear trend during the test segment. Therefore, the data acquisition system for the sediment traps was designed with sufficient capacity to sample the 60 load cells at a frequency of $20 \mathrm{~Hz}$. This rate should provide the capability to quantify the change in sediment transport rate during the early stages of an experiment. In addition, a data acquisition system with this capacity has the possibility to quantify the change in sediment transport rate associated with, for example, a large group of waves in an irregular wave spectrum.

Based on these tentative assumptions, the following measurement methodology is used during a typical experiment. First, all 60 load cells are sampled continuously throughout the entire duration of the test segment. Normally, sand trap data collection begins approximately 1 min prior to wave generation and ends approximately $1 \mathrm{~min}$ after the wave generators are stopped. 
Even though the 60 load cells could be sampled at a frequency of up to $20 \mathrm{~Hz}$, a sampling frequency of $4 \mathrm{~Hz}$ has proven to be sufficient for irregular waves with a peak period of $2.5 \mathrm{sec}$. This provides 10 data points per wave cycle at the peak frequency.

Two smaller data sets also are collected during a typical experiment. The first is collected prior to starting wave generators and vertical turbine pumps at the beginning of the test segment. For this data set, the 60 load cells are sampled at a frequency of $1 \mathrm{~Hz}$ for a duration of $100 \mathrm{sec}$. Another data set is collected in the same manner immediately after the test segment, when the waves and currents have been stopped and the water in the basin has become relatively calm. These two data sets are used to quantify the initial and final submerged weight of sand in each trap.

There are three issues that complicate the task of collecting high quality LST data. The first is the accumulation of air pockets beneath the traps and the neoprene rubber seals. This occurs as the facility is being filled with water, prior to an experiment. However, a simple method was developed to remove these air bubbles from beneath the traps and the neoprene rubber seals, as discussed previously in Chapter 4.

The second issue is related to the observation that some of the sand that is transported into each flow channel accumulates on top of the neoprene rubber seals around the perimeter of the traps. In other words, a portion of the submerged weight of this sand is supported by the walls of the flow channels rather than by the load cells which support the sediment traps. This problem is greatest at the upstream end of the traps, because a significant amount of sand accumulates in that area. To solve this problem, an additional task has been added to the standard operating procedure used to conduct each experiment. The results from a previous LST experiment will be used to illustrate this procedure. In Figure 52, the "PreTest Weight" data are not equal to zero because the traps had not been dredged prior to this test segment. After the test segment was completed, the submerged weight of sand in each trap was measured for $100 \mathrm{sec}$ at a frequency of $1 \mathrm{~Hz}$, as indicated by the "PostTest Weight" data set in the figure. A water hose is used to wash the sand off the rubber seals and into the traps. This task is carried out in two steps. First, the sand that has accumulated on the rubber seals along both sides of the traps is washed into the traps. Then the submerged weight of sand in each trap is measured for the third time, for $100 \mathrm{sec}$ at $1 \mathrm{~Hz}$ and is referred to as the "Side Rubber Cleaned" data set. Next, the sand that has accumulated on the rubber seal at the updrift end of the traps is washed into the traps. Finally, the submerged weight of sand in each trap is measured for the fourth time, for $100 \mathrm{sec}$ at $1 \mathrm{~Hz}$ and is indicated by the "All Rubber Cleaned" data set. This data set represents the true submerged weight in each trap. For this particular test segment, an average of 3.3 and 7.7 percent of the true submerged weight of sand measured in each trap had initially accumulated on the side rubber seals and the updrift rubber seal, respectively. Therefore, a total of 10 percent of the sand had initially accumulated on the rubber seals, which is an average value for the 20 traps. Although this process typically takes $2 \mathrm{hr}$ to complete, it is a crucial step in the process to obtain high quality sediment transport data sets. 


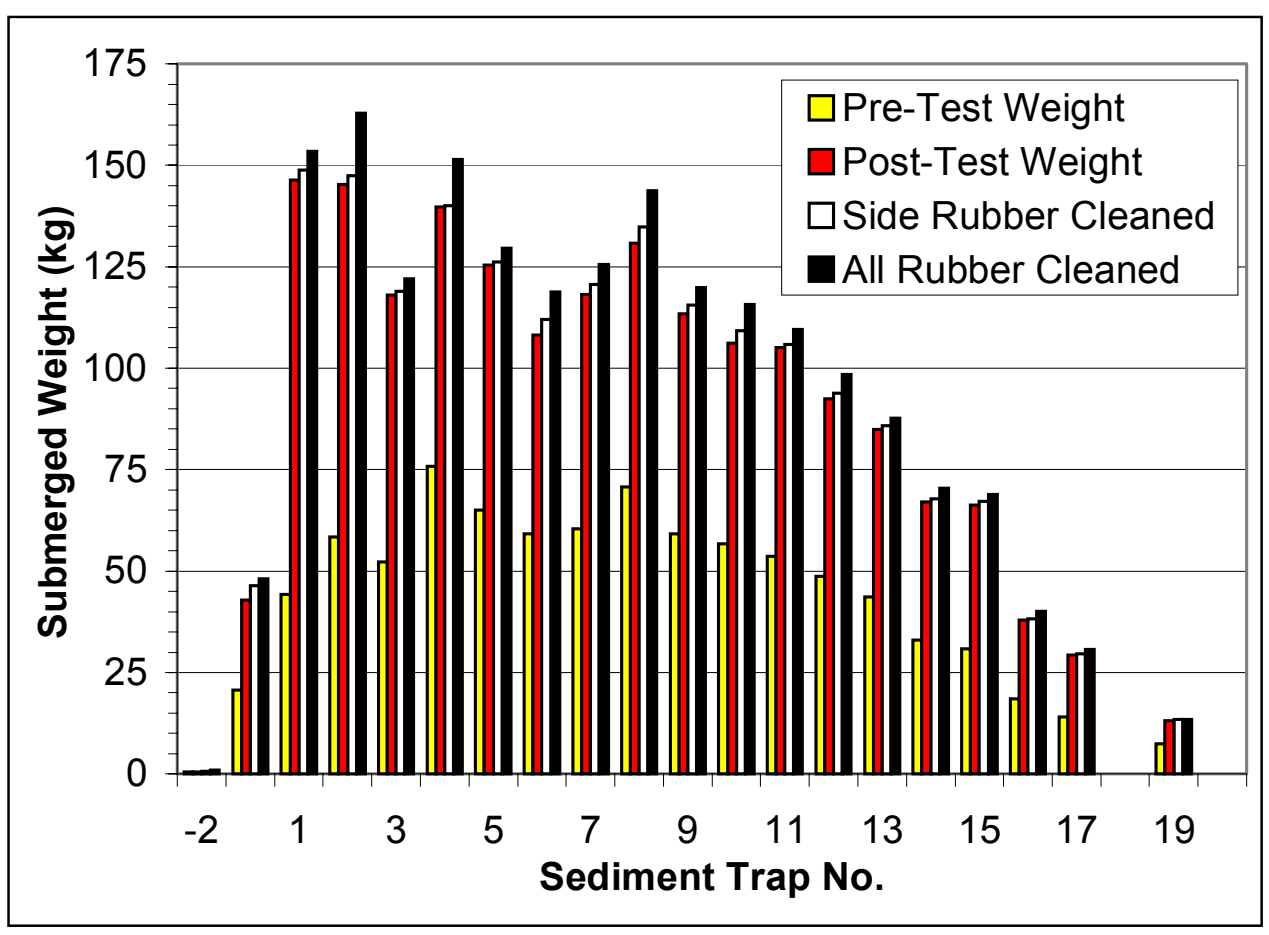

Figure 52. Average sediment trap weights for Test1H_Case2, showing the amount of sand that had accumulated on the neoprene rubber seals

The third issue related to measuring high quality LST data is the observation that, in some cases, a very small amount of sand tends to by-pass some of the sediment traps, even though Sediment Trap No. 1 to 17 and 19 are $6.0 \mathrm{~m}$ in length. This was a concern during the design phase, and every feasible step was taken to maximize the length of the traps. Most importantly, the beach was constructed as far upstream as possible to maximize the length of the downstream flow channels and sediment traps in an effort to provide sufficient time for the suspended sediment to fall out of suspension. Nonetheless, for wave conditions that generate a strong longshore current magnitude near the peak of the distribution, small amounts of sand tend to bypass the traps where the longshore current magnitude is strongest. It should be mentioned that sand bypassing is not a problem where the longshore current magnitude is weaker, for example, farther offshore near the tail of the longshore current distribution. To quantify the magnitude of this problem, several streamer traps (similar to those used by Rosati and Kraus 1989) were installed above the downstream end of the traps during several test segments. The objectives were to quantify the vertical distribution of sediment flux and to integrate this distribution across the $0.75-\mathrm{m}$ width of the flow channel to estimate the total sediment flux past the trap. After collecting data during several test segments, results showed that the maximum quantity of sand bypassing the traps was 1.5 percent of the quantity collected in the sediment trap, which only occurred in the three flow channels with the strongest longshore current magnitude. Because it would be very time consuming to perform these measurements during every experiment, the decision was made to accept this as a potential error in the sediment trap data sets. It should be mentioned that this only occurs near the peak of the longshore current distribution and for 
experiments conducted with relatively energetic wave conditions. For all other cases, the quantity of sand bypassing is negligible.

\section{Analysis of sediment trap data}

The cross-shore distribution of the longshore sediment transport rate measured with the sediment trapping system is calculated by subtracting the "PreTest Weight" data from the "All Rubber Cleaned" data (Figure 52), and dividing by the duration of the test segment. The duration of Test1H_Case 2 was $3.33 \mathrm{hr}$, and results from this calculation are shown in Figure 53. The crossshore location of the center line of each sediment trap is plotted along the x-axis, as tabulated in Table 2. Recall that there are no sediment traps located in Flow Channels No. 18 and 20 (i.e., at cross-shore locations 16.125 and $17.625 \mathrm{~m}$ ). The sand that accumulated in these two flow channels can be visually inspected after the facility is drained. For this test segment, the amount of sand in Flow Channel No. 20 was negligible. This will be the case for most of the experiments conducted in the facility, except for those conducted with very energetic wave conditions. However, there was a small amount of sand in Flow Channel No. 18. This quantity was estimated using linear interpolation between the LST rates measured in Sediment Traps No. 17 and 19. Integrating across-shore gives a total measured LST rate of $314.9 \mathrm{~kg} /$ hour during this test segment, which includes the estimated rate of $3.4 \mathrm{~kg} /$ hour in Flow Channel No. 18.

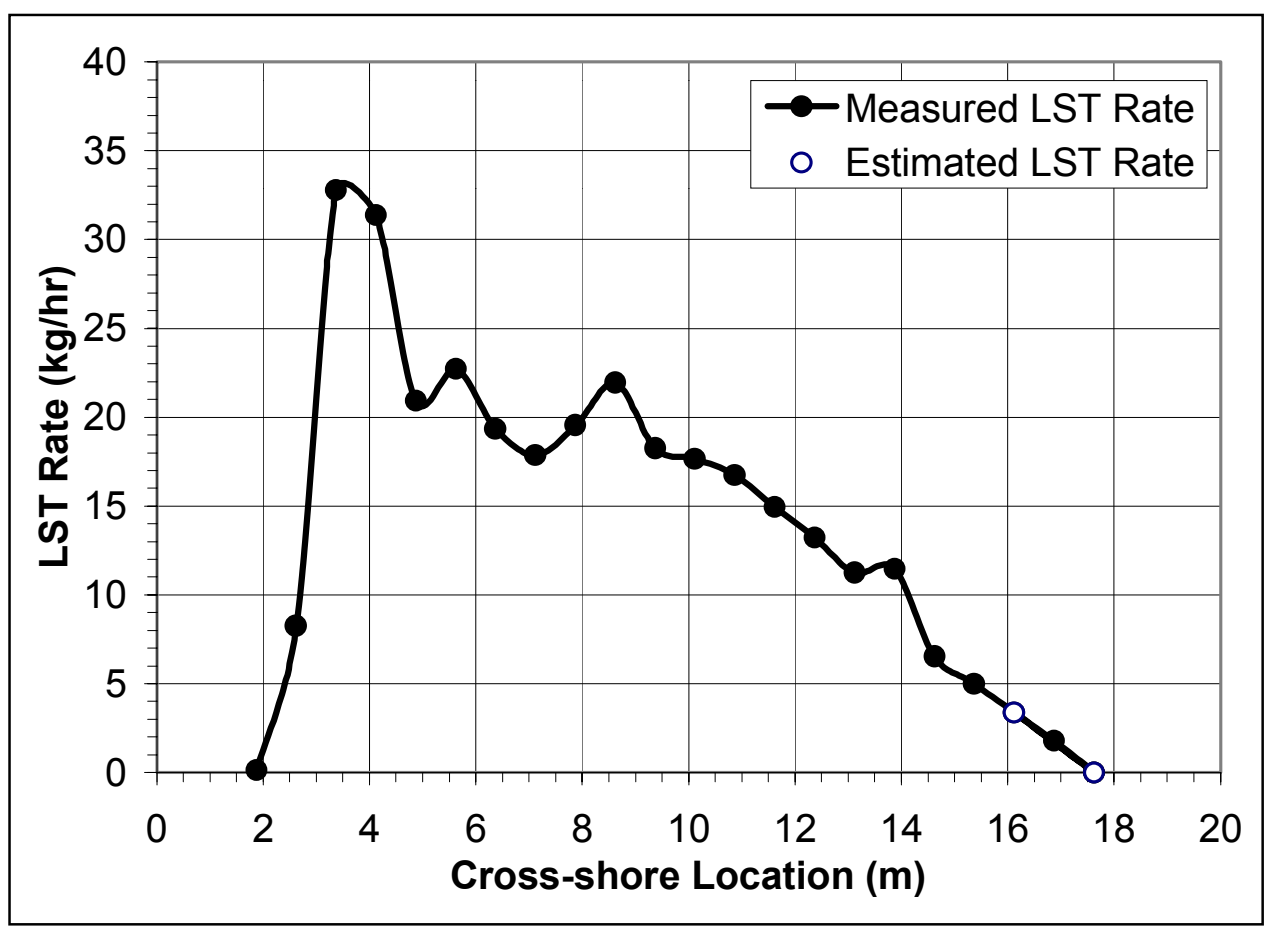

Figure 53. Cross-shore distribution of longshore sediment transport measured during the 3.33-hr duration of test segment Test1H_Case2

One of the objectives of the experiments was to attempt to quantify the change in LST rate during a group of large waves in an irregular wave spectrum. 
Figure 54 shows the instantaneous submerged weights measured with Sediment Trap No. 2 during the first $2 \mathrm{hr}$ of test segment Test1H_Case2, which had a total duration of $3.33 \mathrm{hr}$. Because of a brief delay at the beginning of this test segment, the load cells were sampled for several minutes prior to starting the wave generators. The instantaneous submerged weight measurements contain considerable fluctuation as a result of the vibrations of the sediment traps caused by wave and current forcing. The fluctuations tend to be greatest on the downdrift load cell, where the trap is supported midway across the end of the trap, rather than at both downdrift corners; as is the case for the updrift end of the trap. The reason for this design was discussed previously in Chapter 4. The summation of the three time series also is shown, which represents the total submerged weight of sand in Sediment Trap No. 2. Although the overall trend in the data can be accurately quantified, identification of the influence of large individual waves or groups of waves is not possible because of the noise level in the measured time series.

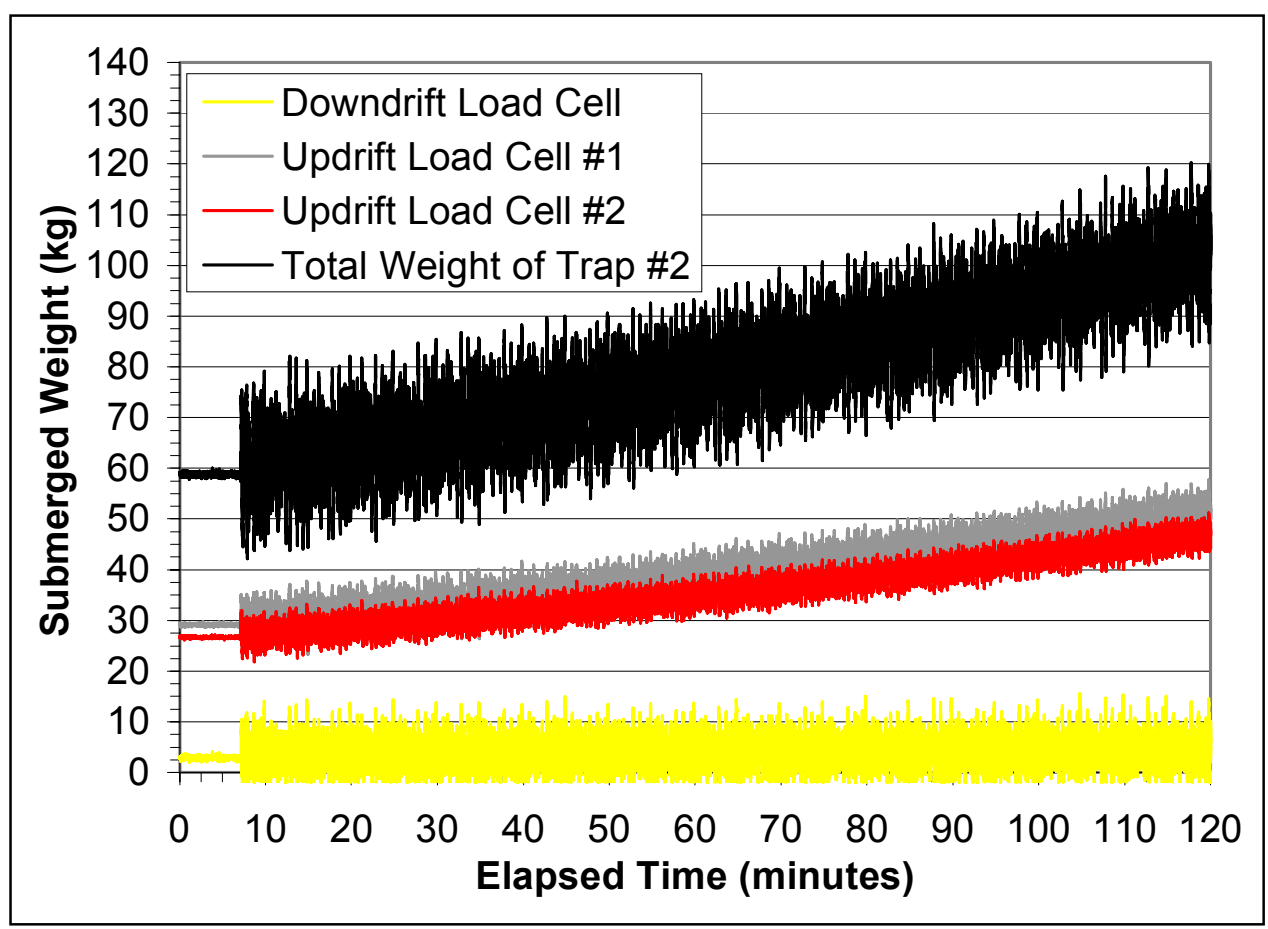

Figure 54. Instantaneous submerged weight of Sediment Trap No. 2 during the first $2 \mathrm{hr}$ of test segment Test1H_Case2

There is one other potential error that must be addressed to obtain highquality longshore sediment transport data sets. During some experiments, a low profile submerged shoal tends to accumulate immediately upstream of the downstream wave guide. This is attributed to imperfections in the downstream lateral boundary, which are essentially unavoidable when conducting moveablebed experiments in the laboratory. More specifically, the cause of this shoal formation is attributed to a decrease in wave energy immediately upstream of the downstream wave guide, resulting from a loss of wave energy under the wave guide. This localized wave height gradient causes a small portion of the suspended sediment to fall out of suspension and slowly accumulate in the form 
of a submerged shoal. The amount of sand that accumulates in this submerged shoal (during the test segment) is quantified volumetrically using the bathymetric data measured immediately prior to and after each test segment. The shoal volume is separated into $0.75-\mathrm{m}$-wide slices running alongshore, and the quantity of sand in each slice is added to the sediment trap immediately downstream of each slice. The method used calculate the quantities will be discussed in detail in Chapter 10.

\section{Water Level Control}

Measuring the still-water level in a laboratory facility is relatively simple, because it can be measured when the fluid is at rest and the water surface is stationary. However, future experiments in the LSTF may involve the simulation of tidal fluctuations, which complicates this measurement. Tides would require simulation with a system to control the inflow and outflow of water. Sophisticated systems have been developed whereby the rate of water level change in the model is controlled by an automated system that relies on feedback from the water level gauge (Hughes 1993). Although a system to simulate tides does not presently exist in the LSTF, a water level control gauge was built to make this possible in the future.

Because of the long-term nature of conducting sediment transport experiments in a large-scale laboratory facility, it is important to maintain a known water level during several months (or possibly a few years) of laboratory studies. For example, unknown variations in the still-water level can cause undesirable variations in the offshore wave height from one experiment to the next. Therefore, a simple but robust system was constructed to provide an accurate and consistent method of measuring the still-water level in the facility over long periods of time.

A custom designed graduated glass cylinder was built for the LSTF and was mounted inside of a protective support frame on the outside of the exterior wall of the facility. The graduated cylinder has an inside diameter of $0.1 \mathrm{~m}$. A polyurethane tube, with an inside diameter of $0.003 \mathrm{~m}$, connects the graduated cylinder to the reservoir of water in the facility. Therefore, the surface area of the water in the graduated cylinder is three orders of magnitude larger than the cross-sectional area of the inflow/outflow tube. This was done to essentially eliminate water surface elevation fluctuations while waves are generated. Therefore, the graduated cylinder would be ideal for measuring the mean water level in the facility if tides are simulated in the future. The zero reading on the graduated cylinder was set at the average elevation of the concrete floor in the facility in the region where waves are generated. By considering the meniscus of the water surface on the inside face of the graduated cylinder, the water level in the facility can be maintained within $\pm 1 \mathrm{~mm}$ of the desired water level.

\section{Summary}

The LSTF is a high-quality laboratory facility that has been designed with the capability to measure wave, current, sediment concentration, bathymetric, 
and longshore sediment transport data. The longshore sediment transport data measured with the sediment trapping system is unique in that there are a total of 20 data points that accurately define the cross-shore distribution. Furthermore, each of these 20 data points can be correlated with the wave height dissipation, vertical longshore current profile, vertical sediment concentration profile, and the local water depth measured at each of these cross-shore locations. These five high-quality data sets provide a tremendous amount of information in regard to the hydrodynamic and sediment transport processes that are generated by forcing from incident waves. The procedures discussed in this chapter have been tested and improved upon over time and have proven to be efficient methods of obtaining high-quality data sets in the LSTF. 


\section{Establishing Uniform Longshore Currents}

\section{Introduction}

Successful execution of sediment transport experiments requires a method for establishing the proper longshore current for a given incident wave condition. The term "proper" longshore current is used to describe the longshore current that is generated along an infinitely long beach having a cross section and an incident wave forcing that are invariant in the longshore direction. As discussed in Chapter 3, an active pumping and recirculation system was developed for the LSTF to establish the proper longshore current.

The external recirculation system and procedures for operating it should maximize the length of beach for which waves, currents, sediment transport, and beach morphology are nearly uniform in the alongshore direction. Longshore uniformity is important both in regions where wave, current, and sediment concentration data are collected, and at the downdrift boundary of the beach where the sediment traps are located. Creating the proper longshore current with a high degree of longshore uniformity is difficult to achieve. The capability of the LSTF recirculation system to meet these objectives is the subject of this chapter. Two comprehensive series of longshore current experiments were performed on the fixed concrete beach to facilitate evaluation of the system. The experiments were conducted as a precursor to a more complex series of moveable-bed longshore sediment transport experiments. The majority of the material discussed in this chapter was published by Hamilton and Ebersole (2001).

\section{Experiment Program}

A number of preliminary experiments were conducted to investigate: (a) long-term oscillations in pump discharge rates (found to be negligible); (b) flow patterns created by pumping only (i.e., no wave forcing); (c) flow patterns with waves only (i.e., no external current recirculation); and (d) the time required for mean velocities in the wave basin to reach steady state.

After these preliminary experiments were completed, two comprehensive test series were conducted, one using regular waves and the other using irregular 
waves. Figure 55 is a photograph of the LSTF taken during a regular wave experiment. A total of 20 experiments were conducted, each with a different magnitude and cross-shore distribution of pumped flow rate. Fifteen regular wave experiments were conducted in the process of determining the proper magnitude and cross-shore distribution of the longshore current and were identified as Test 2 (waves only) and Tests 6A-6N (waves and currents). Five irregular wave experiments were conducted and identified as Tests 8A-8E.

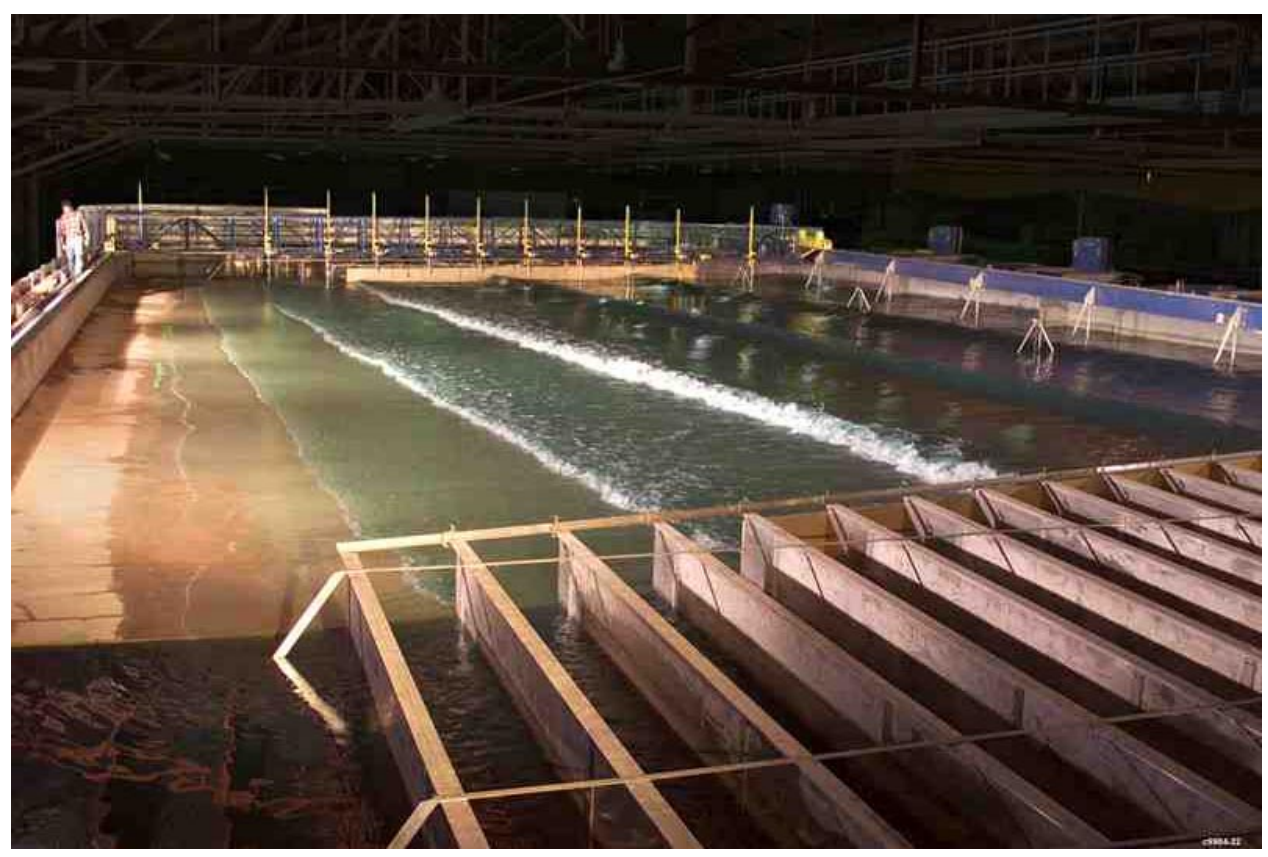

Figure 55. Oblique view of the Large-scale Sediment Transport Facility (regular wave experiment). A model operator standing in the upper left corner indicates the physical size of the facility

The incident wave conditions adopted for Tests $6 \mathrm{~A}-\mathrm{N}$ and Tests $8 \mathrm{~A}-\mathrm{E}$ are given in Table 4, where $\mathrm{T}$ is wave period (peak spectral wave period, $\mathrm{T}_{\mathrm{p}}$, for irregular wave case), $\mathrm{H}$ is wave height (energy-based significant wave height, $H_{\text {mo }}$, for irregular wave case), $\lambda$ is wave length, $d$ is still-water depth, $\theta$ is the angle of incidence relative to shore normal, and the subscripts " 0 " and " 1 " refer to values in deep water and at the wave generators, respectively. Deepwater values were calculated using linear wave theory. For the irregular wave tests, $\mathrm{H}_{\text {mo }}$ was selected so that the root-mean-square wave height, $\mathrm{H}_{\mathrm{rms}}$, was comparable to the average wave height, $\mathrm{H}_{\text {avg }}$, for the regular wave case. Therefore, the total incident wave energy used for both regular and irregular waves was similar. For the irregular wave tests, a TMA spectrum was used to define the spectral shape. The spectral width parameter was 3.3, a value representing typical wind sea conditions. A random phase method was used to synthesize the pseudo-random wave train used to drive the wave generators. The length of the drive signal was $500 \mathrm{sec}$, duration of 200 times the peak wave period $(2.5 \mathrm{sec})$. The still-water depth at the wave generators was constant at $0.667 \mathrm{~m}$ during all fixed-bed experiments. 


\begin{tabular}{|c|c|c|c|c|c|c|c|c|c|}
\hline Test & $\begin{array}{l}\text { Wave } \\
\text { Type }\end{array}$ & $\begin{array}{l}\mathrm{T} \\
\text { sec }\end{array}$ & $\begin{array}{l}\mathrm{H}_{1} \\
\mathrm{~m}\end{array}$ & 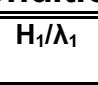 & $\begin{array}{l}d_{1} \\
m\end{array}$ & $\begin{array}{l}\Theta_{1} \\
\text { deg }\end{array}$ & $\begin{array}{l}\mathrm{H}_{0} \\
\mathrm{~m}\end{array}$ & $\overline{\mathbf{H}_{0} / \boldsymbol{\lambda}_{0}}$ & $\begin{array}{l}\Theta_{0} \\
\text { deg }\end{array}$ \\
\hline 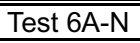 & Regular & 2.5 & 0.182 & 0.031 & 0.667 & 10.0 & 0.189 & 0.019 & 16.6 \\
\hline Test 8A-E & \begin{tabular}{|l|} 
Irregular \\
\end{tabular} & 2.5 & 0.225 & 0.038 & 0.667 & 10.0 & 0.233 & 0.024 & 16.6 \\
\hline
\end{tabular}

\section{Measurement Methodology}

The instrumentation bridge was used to position the wave and current sensors at various positions along the beach. Transect locations were selected every $4.0 \mathrm{~m}$, from $\mathrm{y}=15.0$ to $\mathrm{y}=39.0 \mathrm{~m}$, as shown in Figure 56. Transects are identified as Y15, Y19, Y23, Y27, Y31, Y35, and Y39, according to their longshore coordinate. During each experiment, measurements were taken along at least three primary transects to represent general hydrodynamic conditions along the beach: Y19 (center of the downstream half of the beach), Y27 (center of the entire beach), and Y35 (center of the upstream half of the beach). During Test $6 \mathrm{~N}$ and Test $8 \mathrm{E}$, and several others, measurements were made at all seven transects.

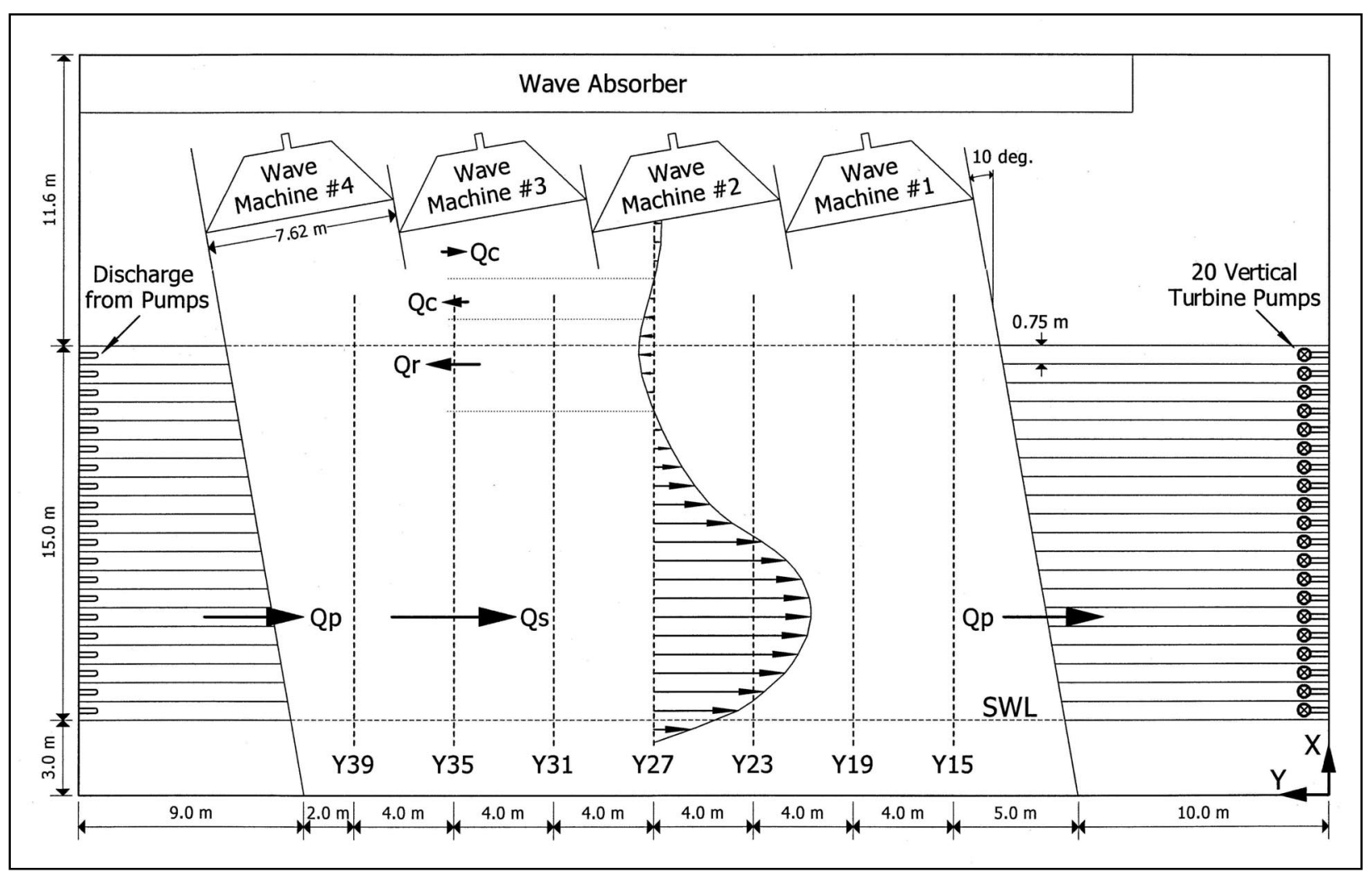

Figure 56. Plan view of the LSTF and a conceptual diagram of the longshore flow conditions

Wave setup and setdown were obtained by using the wave gauges to measure the still-water level elevation prior to each experiment and then subtracting that elevation from the mean water surface elevation measured during the experiment. 
The ADVs were positioned at elevations approximately one-third of the water depth above the bed.

At the beginning of each experiment, the instrumentation bridge was positioned at Y27, and the elevation of the still-water level was measured with the 14 wave gauges. The pumps were started and set to prescribed discharges to create the desired longshore current distribution. Data collected with the in-line flow sensors (one in each of the 20 pump-and-piping systems) were analyzed to ensure the pumps were operating at the proper flow rates. The wave generators were started and waves were generated continuously throughout the experiment. After $10 \mathrm{~min}$ of wave generation (20 min after the pumps were started), data were collected at the Y27 transect. All sensors were sampled at $20 \mathrm{~Hz}$ for 500 sec during both the regular and irregular wave test series. The process of repositioning the instrumentation bridge and acquiring $500 \mathrm{sec}$ of data was repeated at transects Y15, Y19, Y23, Y27, Y31, Y35, and Y39. After the last transect was completed at Y39, a third set of data was acquired at Y27. Multiple measurements at transect Y27 were collected to assess repeatability of the measurements and the steadiness of the hydrodynamic conditions.

The time series were visually inspected during experiments to assess data quality. In very shallow water, air bubbles from breaking waves penetrated into the water column, to the depth of the ADV 1 and 2 sensors, causing undesirable spikes in the velocity time series. These spikes were removed during postprocessing with a filtering routine developed and discussed in Chapter 8 . The ADV measurements further offshore did not need to be filtered.

Detailed dye measurements were performed during each experiment to inspect patterns in the flow streamlines by injecting the dye into the water at discrete points. Dye observations focused on: (a) uniformity of flow streamlines along the beach; (b) streamline patterns of flow exiting the upstream flow channels and approaching the downstream flow channels; and (c) streamlines in the offshore region of the basin where internal recirculation occurred. Dye also was used to obtain qualitative information on the longshore current in very shallow water, shoreward of ADV 1.

\section{Procedure for Tuning the Longshore Current}

This section contains three subsections. The first describes the iterative process that was used to establish the proper magnitude and cross-shore distribution of the longshore current along the beach by adjusting the pump settings for the external recirculation system. To verify these results, the two subsequent subsections describe two criteria proposed by Visser to confirm that the proper total longshore flow rate was recirculated. However, these two criteria consider only the magnitude of the total longshore flow rate. They do not help determine the proper cross-shore distribution of the longshore current that should be recirculated.

Figure 56 conceptually illustrates the flow conditions in the LSTF during the experiments. The quantity $\mathrm{Q}_{\mathrm{s}}$ is the total longshore flow rate in the surf zone between the wave setup limit and the point of transition where the mean 
longshore current reverses direction; $\mathrm{Q}_{\mathrm{p}}$ is the total longshore flow rate actively pumped through the external recirculation system; $Q_{r}$ is the total longshore flow rate that internally recirculates in the offshore region; and $\mathrm{Q}_{\mathrm{c}}$ is a secondary offshore circulation cell limited to the length of each wave board between two adjacent baffles. In this facility, $Q_{c}$ develops as a result of the baffles that extend shoreward of the wave boards. In general, it was found that $\mathrm{Q}_{\mathrm{c}}$ decreases as $\mathrm{Q}_{\mathrm{r}}$ decreases, because $\mathrm{Q}_{\mathrm{r}}$ drives $\mathrm{Q}_{\mathrm{c}}$. At a transect midway along the beach, and assuming no temporal change in mean water level within the wave basin,

$$
\mathrm{Q}_{\mathrm{s}}=\mathrm{Q}_{\mathrm{p}}+\mathrm{Q}_{\mathrm{r}}
$$

\section{Iterative examination of the longshore current distribution}

Pump settings were systematically adjusted in an effort to establish the proper mean longshore current distribution in the surf zone for a given wave condition. As the series of experiments progressed, new estimates of the proper longshore current distribution were made based on previously measured distributions along the beach. The following hypotheses were used to guide the tuning process: (a) the degree of uniformity of longshore current in the surf zone should increase as the proper longshore current distribution is approached; (b) the magnitude of internal recirculation, $\mathrm{Q}_{\mathrm{r}}$, should decrease as the proper longshore current distribution is approached; (c) there is a point where $\mathrm{Q}_{\mathrm{r}}$ is minimized; and (d) internal recirculation can not be completely eliminated because of imperfections of the lateral boundaries.

The first regular wave experiment, Test 2, was conducted with no pumping $\left(\mathrm{Q}_{\mathrm{p}}=0 \ell / \mathrm{sec}\right)$. This test was conducted to investigate the case when $\mathrm{Q}_{\mathrm{r}}$ had the largest magnitude and to examine the signs of under-pumping. This test is equivalent to the recirculation scheme used by Putnam, Munk, Traylor (1949), and discussed by Visser (1991) and Hamilton et al. (1997). As expected, results from Test 2 showed very nonuniform conditions both in the magnitude and distribution of longshore current measured at Y19, Y27, and Y35.

A total of 14 regular wave experiments were conducted with $\mathrm{Q}_{\mathrm{p}}>0 \mathrm{l} / \mathrm{sec}$. An initial estimate of the proper longshore current distribution was made using the numerical model NMLONG (Kraus and Larson 1991). The breaking wave height-to-depth ratio, used as a calibration parameter in the numerical model, was adjusted based on wave height measurements in the fixed-bed experiments.

Figure 57 shows pump settings for 7 of the 14 experiments, including the proper distribution, Test $6 \mathrm{~N}$, for comparison. Pump settings are shown in terms of the depth-averaged longshore current pumped through the lateral boundaries of the facility. As illustrated, the proper longshore current distribution was achieved by under-pumping across the entire surf zone and gradually increasing the pumped flow rate through the lateral boundaries. 


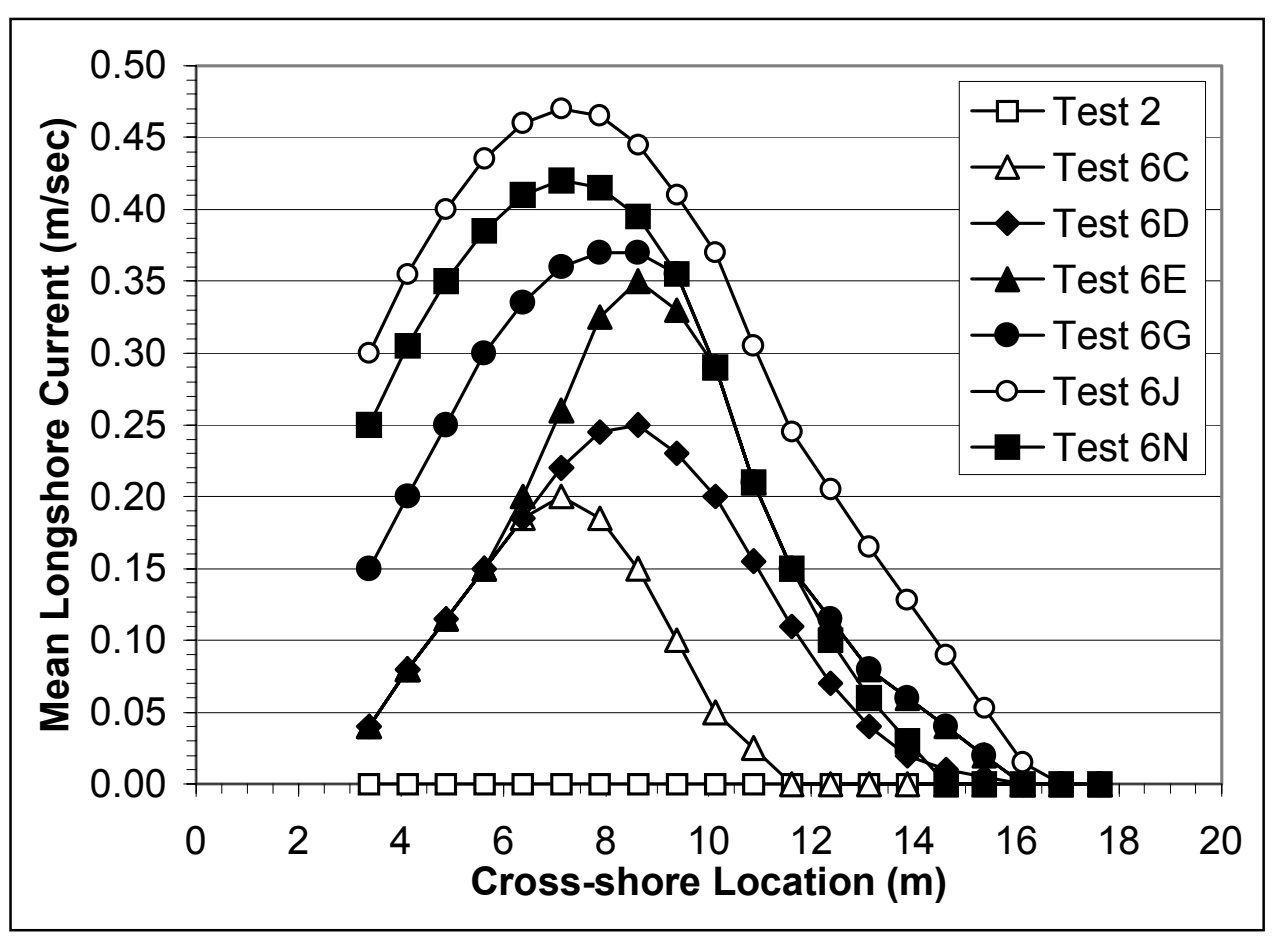

Figure 57. Progression of pump settings for regular wave experiments

Results from Test 6D, an under-pumped case that was the fifth experiment in the series, are shown in Figure 58. The peak longshore current measured at each of the three transects was much higher than the peak current pumped through the lateral boundaries. Offshore recirculation was diminished substantially in both extent and magnitude compared to the no-pumping case; however, recirculation remained rather strong at Y27. The longshore current distribution at Y35 showed a region of flow reversal near the shoreline (negative values). This phenomenon was observed during the 15 experiments and was limited within a region extending from the upstream boundary to $Y=34 \mathrm{~m}$ and $2 \mathrm{~m}$ offshore from the still-water shoreline. This region of flow reversal decreased significantly in magnitude and spatial extent as the proper distribution was approached with subsequent pump settings, but it was never completely eliminated.

It appeared that the proper longshore current distribution was being approached at the peak and shoreward of the peak during the tenth experiment (Test 61). However, there was concern that the offshore tail of the distribution was over-pumped. To investigate the ramifications and signs of over-pumping, Test $6 \mathrm{~J}$ was performed. Figure 57 shows the longshore current distribution that was recirculated in Test $6 \mathrm{~J}$, relative to other tests, and Figure 59 shows longshore current results from the test. In the surf zone, the measured current distribution matched the pump settings quite well. In this region, it would be difficult to discern whether or not the lateral boundaries were over-pumped. However, the over-pumped case (Test $6 \mathrm{~J}$ ) produced substantially more recirculation in the offshore region, relative to Test 6I. The results from this experiment indicated that there are signs of over pumping, but the results are subtle and not obvious over much of the longshore current distribution, except in the offshore tail. 


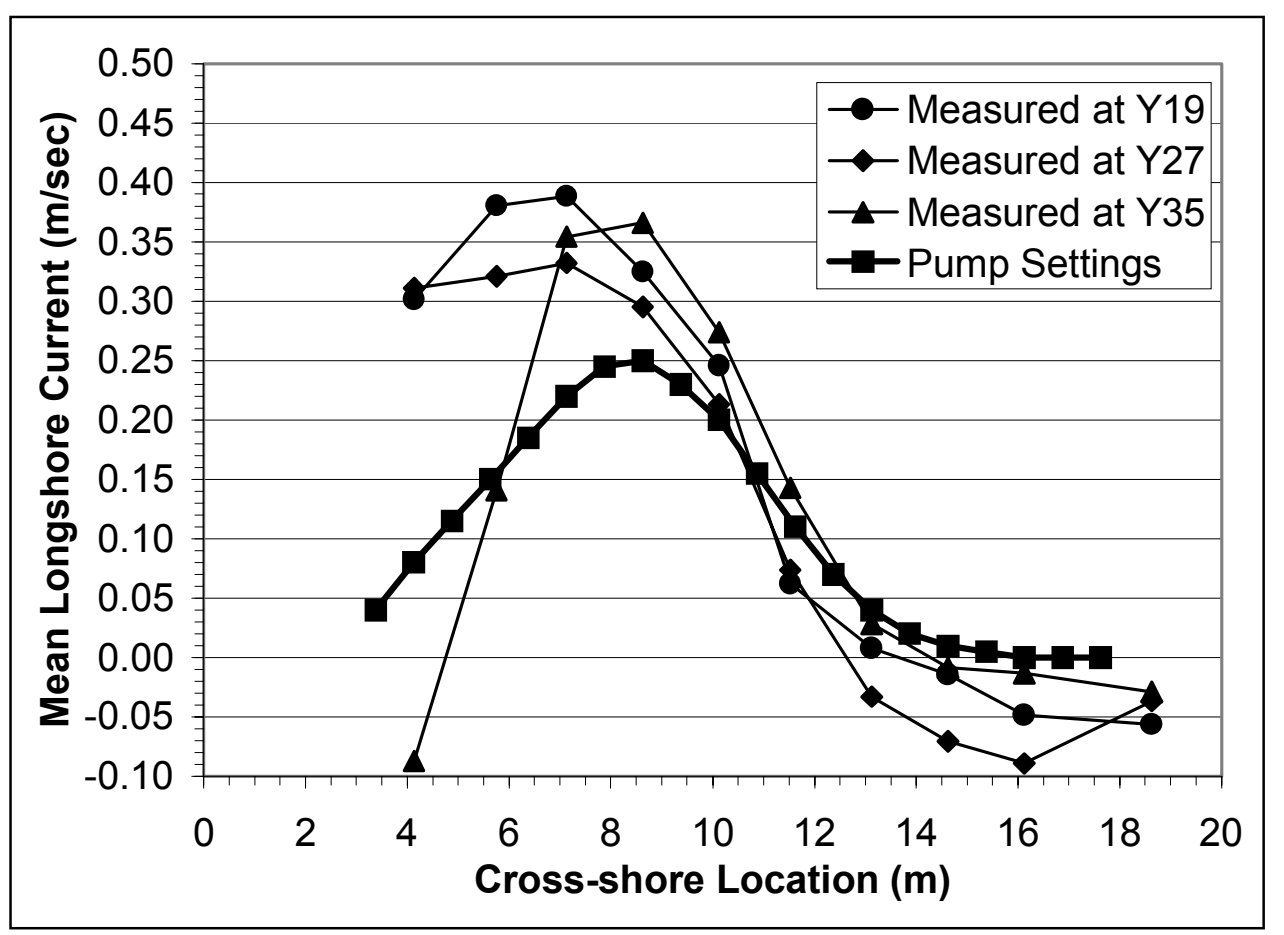

Figure 58. Test 6D - under-pumping: Measured longshore current distirbution at $\mathrm{Y}-19,27$, and $35 \mathrm{~m}$

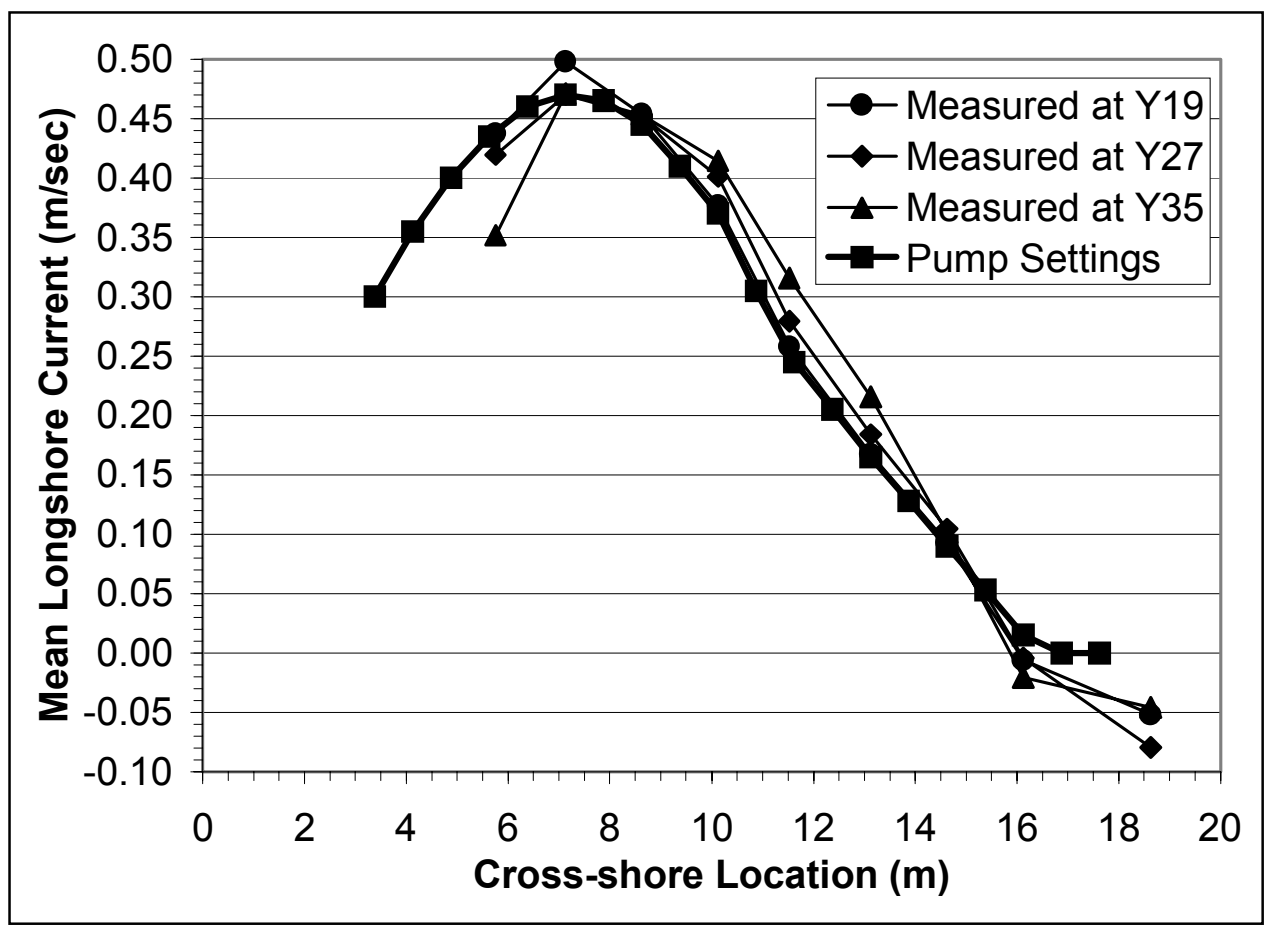

Figure 59. Test $6 \mathrm{~J}$ - over-pumping: Measured longshore current distribution at $\mathrm{Y}=19,27$, and $35 \mathrm{~m}$ 
The last few experiments ( $6 \mathrm{~K}$ through $6 \mathrm{~N}$ ) focused in more detail on the offshore tail of the distribution, which proved to be more difficult to tune. Figure 60 shows the results from Test $6 \mathrm{~N}$, the fifteenth and final iteration. Peak currents at the three primary transects were relatively uniform, and the degree of uniformity along much of the beach increased noticeably. In the offshore tail, agreement between pump settings and measurements was the best observed in any prior experiment. Current measurements in the offshore tail always were slightly greater than the pump settings, because of the internal recirculation. The magnitude and extent of the small flow reversal region near the shoreline at the upstream end of the beach was significantly less, and the longshore current measured closest to the shoreline at Y35 was now directed downstream (positive values). However, the flow reversal near the shoreline was still present farther upstream. The flow reversal appeared to cause the higher current magnitude measured immediately offshore of the peak at Y35. The internal recirculation, $\mathrm{Q}_{\mathrm{r}}$ was the lowest that had been observed during all previous tests, based on the measurements at the ADV furthest offshore. Therefore, it was concluded that the pump settings used during Test $6 \mathrm{~N}$ were the proper settings, and they produced the highest degree of uniformity of longshore currents in the surf zone.

Additional measurements were made offshore at the Y27 transect to more accurately quantify the magnitude of the internal recirculation in the offshore region. They are discussed in more detail in the next subsection.

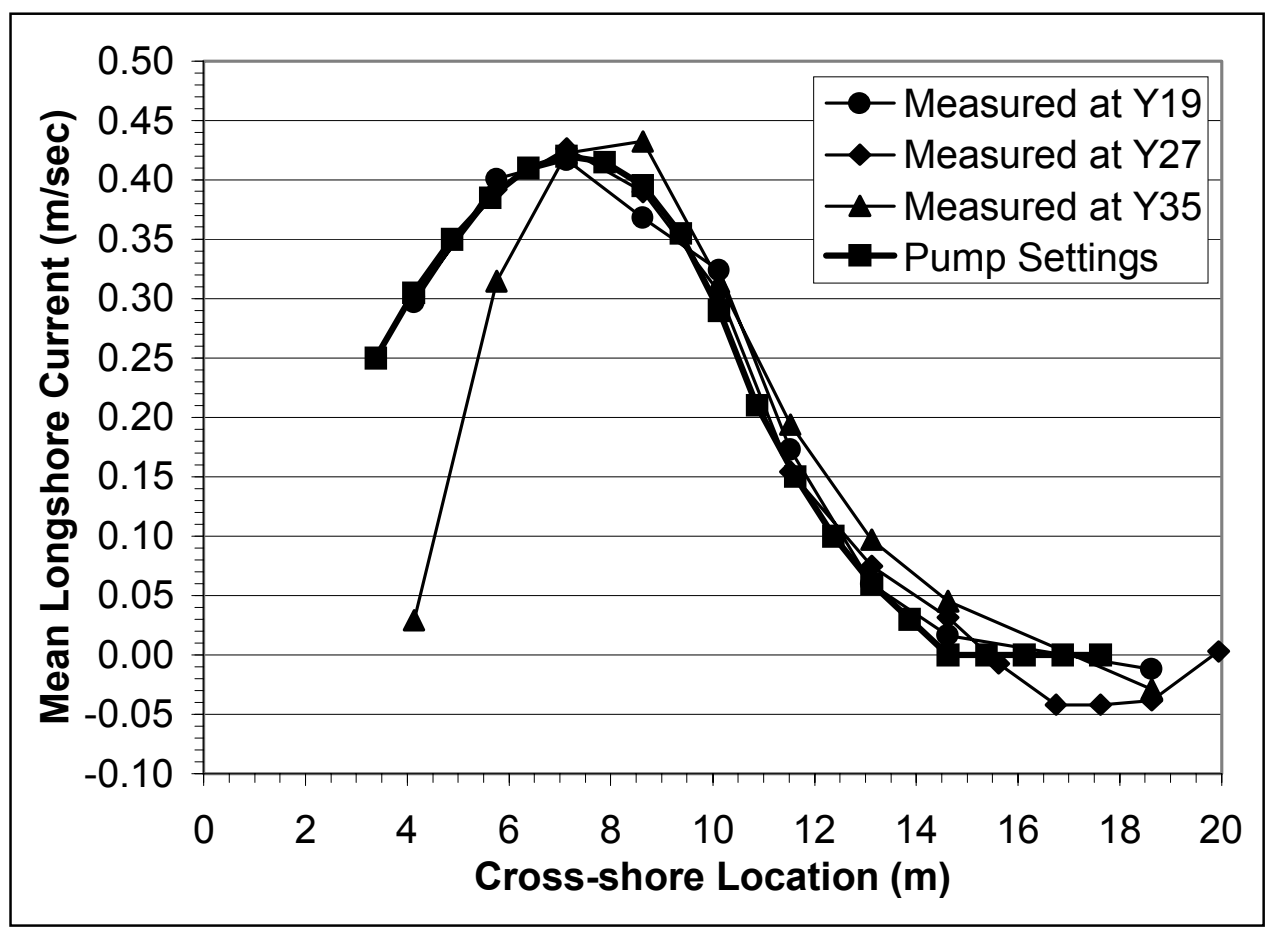

Figure 60. Test $6 \mathrm{~N}$ - proper pumping: Measured longshore current distribution at $\mathrm{Y}=19,27$, and $35 \mathrm{~m}$

The same iterative process used for the regular wave case was repeated for the irregular wave case. The final longshore current distribution was achieved in Test $8 \mathrm{E}$, after five iterations. A more complete set of results that illustrates the 
degree of longshore uniformity that was achieved, are presented and discussed in a subsequent section and provided in tabular form in Appendix A.

\section{Verification using minimum $Q_{r}$ concept}

The values of $\mathrm{Q}_{\mathrm{p}}$ used during Test $6 \mathrm{~N}$ and Test $8 \mathrm{E}$, assumed to be the optimum values, were verified using the two criteria proposed by Visser (1991). As mentioned previously, these two criteria consider only the total longshore flow rate being recirculated, $Q_{p}$, not the cross-shore distribution (i.e., not the 20 individual pump flow rates).

In the present experiments, $\mathrm{Q}_{\mathrm{r}}$, which is influenced by $\mathrm{Q}_{\mathrm{c}}$ (Figure 56), was estimated directly during Tests $6 \mathrm{~N}$ and Test $8 \mathrm{E}$, by making additional measurements in the offshore region at transect Y27. For all other experiments in the Test 6 and Test 8 series, $Q_{r}$ was estimated indirectly assuming $Q_{r}=Q_{s}-Q_{p}$ (Equation 1). Quantifying the magnitude of $\mathrm{Q}_{\mathrm{c}}$ was difficult and could only be estimated using dye.

As shown in Appendix A, the mean longshore currents were rather invariant with depth. Therefore, the flow rate in the surf zone, $\mathrm{Q}_{\mathrm{s}}$, could be calculated using the longshore current measured one-third of the water depth above the bottom, the local mean water surface elevation, and an estimate of the crosssectional area represented by each ADV. The pumped flow rate, $Q_{p}$, was calculated using data from the in-line flow sensors in each pump-and-piping system.

The first criterion proposed by Visser (1991) will be illustrated conceptually, using the $\mathrm{Q}_{\mathrm{s}}$ and $\mathrm{Q}_{\mathrm{r}}$ values obtained during the 15 regular wave experiments (Figure 61). $\mathrm{Q}_{\mathrm{pu}}$ is the value of $\mathrm{Q}_{\mathrm{p}}$ associated with the proper and nearly uniform longshore flow rate in the surf zone, $Q_{\mathrm{su}}$. This method is based on the premise that $\mathrm{Q}_{\mathrm{pu}}$ can be determined by minimizing $\mathrm{Q}_{\mathrm{r}}$ as a function of $\mathrm{Q}_{\mathrm{p}}$. Visser (1991) postulated the following: (a) if $\mathrm{Q}_{\mathrm{p}}<\mathrm{Q}_{\mathrm{pu}}$, the flow rate $\mathrm{Q}_{\mathrm{s}}$ will increase in the downstream direction and the surplus, $\mathrm{Q}_{\mathrm{s}}-\mathrm{Q}_{\mathrm{p}}$, will return offshore and increase $\mathrm{Q}_{\mathrm{r}}$; or (b) if $\mathrm{Q}_{\mathrm{p}}>\mathrm{Q}_{\mathrm{pu}}$, the increased flow rate $\mathrm{Q}_{\mathrm{s}}$ will increase $\mathrm{Q}_{\mathrm{r}}$ offshore because of the increased advection and lateral friction.

Although there is some scatter in the data, Test $6 \mathrm{~N}$ had the lowest value of $\mathrm{Q}_{\mathrm{r}}$. For Test $6 \mathrm{~N}, \mathrm{Q}_{\mathrm{s}}$ and $\mathrm{Q}_{\mathrm{p}}$ were 505 and $465 \mathrm{\ell} / \mathrm{sec}$, respectively. Hence $\mathrm{Q}_{\mathrm{r}}$ is indirectly estimated to be $40 \mathrm{l} / \mathrm{sec}$. Based on ADV measurements, the internal recirculation flowing in the upstream direction, $\mathrm{Q}_{\mathrm{r}}+\mathrm{Q}_{\mathrm{c}}$, was calculated to be $48 \mathrm{l} / \mathrm{sec}$. The secondary circulation cell, $\mathrm{Q}_{\mathrm{c}}$, flowing downstream directly in front of each wave generator was estimated to be $10 \mathrm{l} / \mathrm{sec}$, using dye. Therefore, the inferred value of $Q_{r}$ was $38 \mathrm{l} / \mathrm{sec}$. Hence, direct measurement of the internal recirculation, $\mathrm{Q}_{\mathrm{r}}$, and the indirect estimate, $\mathrm{Q}_{\mathrm{s}}-\mathrm{Q}_{\mathrm{p}}$, gave good agreement. The ability to minimize internal recirculation relative to the flow rate in the surf zone, can be quantified as $\left(\mathrm{Q}_{\mathrm{s}}-\mathrm{Q}_{\mathrm{p}}\right) / \mathrm{Q}_{\mathrm{s}}$, which for this test was approximately 8 percent. 


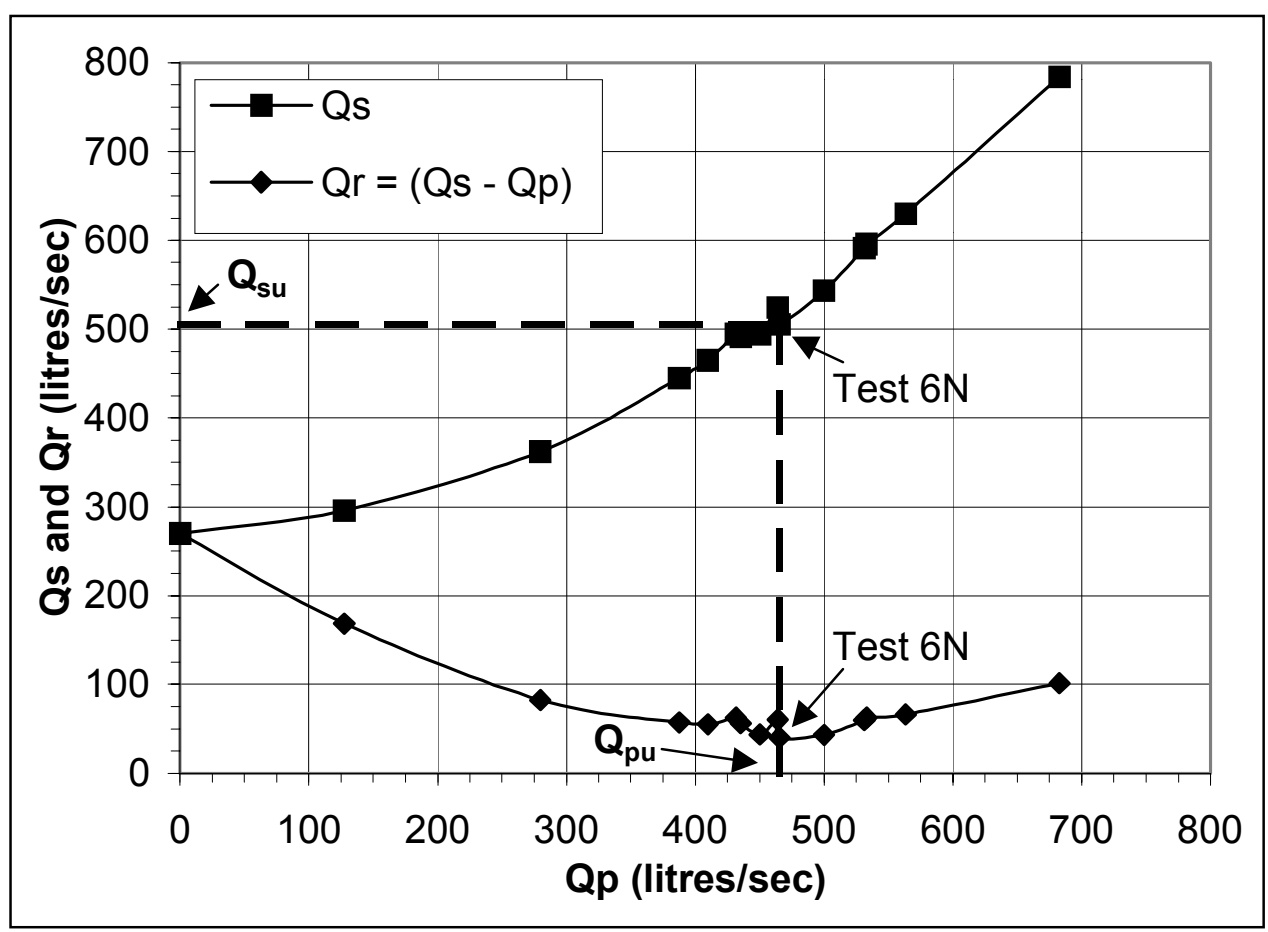

Figure 61. Surf zone and internal recirculation flow rates at $\mathrm{Y} 27$ for 15 regular wave experiments

Values of $\mathrm{Q}_{\mathrm{s}}$ and $\mathrm{Q}_{\mathrm{r}}$ for the five irregular wave experiments are shown in Figure 62. The slight upward curvature in the $\mathrm{Q}_{\mathrm{r}}$ curve is evidence that $\mathrm{Q}_{\mathrm{p}}$ had been increased sufficiently to reach the minimum value of $Q_{r}$, perhaps even slightly exceeding the proper flow rate, $\mathrm{Q}_{\mathrm{pu}}$, in Test $8 \mathrm{E}$ (the largest value of $\mathrm{Q}_{\mathrm{p}}$ ). However, evidence provided in the next section suggests that $\mathrm{Q}_{\mathrm{p}}$ may have been slightly less than $\mathrm{Q}_{\mathrm{pu}}$. For Test $8 \mathrm{E}, \mathrm{Q}_{\mathrm{s}}$ and $\mathrm{Q}_{\mathrm{p}}$ were calculated to be 545 and $478 \mathrm{l} / \mathrm{sec}$, respectively. Hence $\mathrm{Q}_{\mathrm{r}}$ is indirectly estimated to be $67 \mathrm{\ell} / \mathrm{sec}$. Based on ADV measurements, $\mathrm{Q}_{\mathrm{r}}+\mathrm{Q}_{\mathrm{c}}$ was calculated to be $135 \mathrm{\ell} / \mathrm{sec}$. Based on dye measurements, $Q_{c}$ was estimated to be 60 to $70 \mathrm{l} / \mathrm{sec}$, flowing downstream directly in front of each wave generator. Therefore, the inferred value of $\mathrm{Q}_{\mathrm{r}}$ was 65 to $75 \mathrm{l} / \mathrm{sec}$, and the value of $\mathrm{Q}_{\mathrm{r}}$ and $\mathrm{Q}_{\mathrm{c}}$ are comparable. Hence, both the direct and indirect measurements of the internal recirculation, $\mathrm{Q}_{\mathrm{r}}$, were in relatively good agreement. For Test $8 \mathrm{E}$ the ratio of $\left(\mathrm{Q}_{\mathrm{s}}-\mathrm{Q}_{\mathrm{p}}\right) / \mathrm{Q}_{\mathrm{s}}$ was approximately 12 percent. More internal recirculation was generated in the irregular wave case because 2 to 3 percent of the waves broke slightly offshore of $x=18 \mathrm{~m}$, the offshore limit of the external recirculation system.

Results for both the regular and irregular wave test series suggest that the recirculation criteria proposed by Visser (1991) is valid for the LSTF. However, for the LSTF, the gradient in the $\mathrm{Q}_{\mathrm{s}}-\mathrm{Q}_{\mathrm{p}}$ curve tends to increase more gradually as $\mathrm{Q}_{\mathrm{p}}$ is increased, compared with the results presented by Visser (1991). Figures 61 and 62 both suggest that $Q_{p}$ could vary by as much as \pm 20 percent, relative to $Q_{p u}$, without a significant increase in $Q_{r}$. 


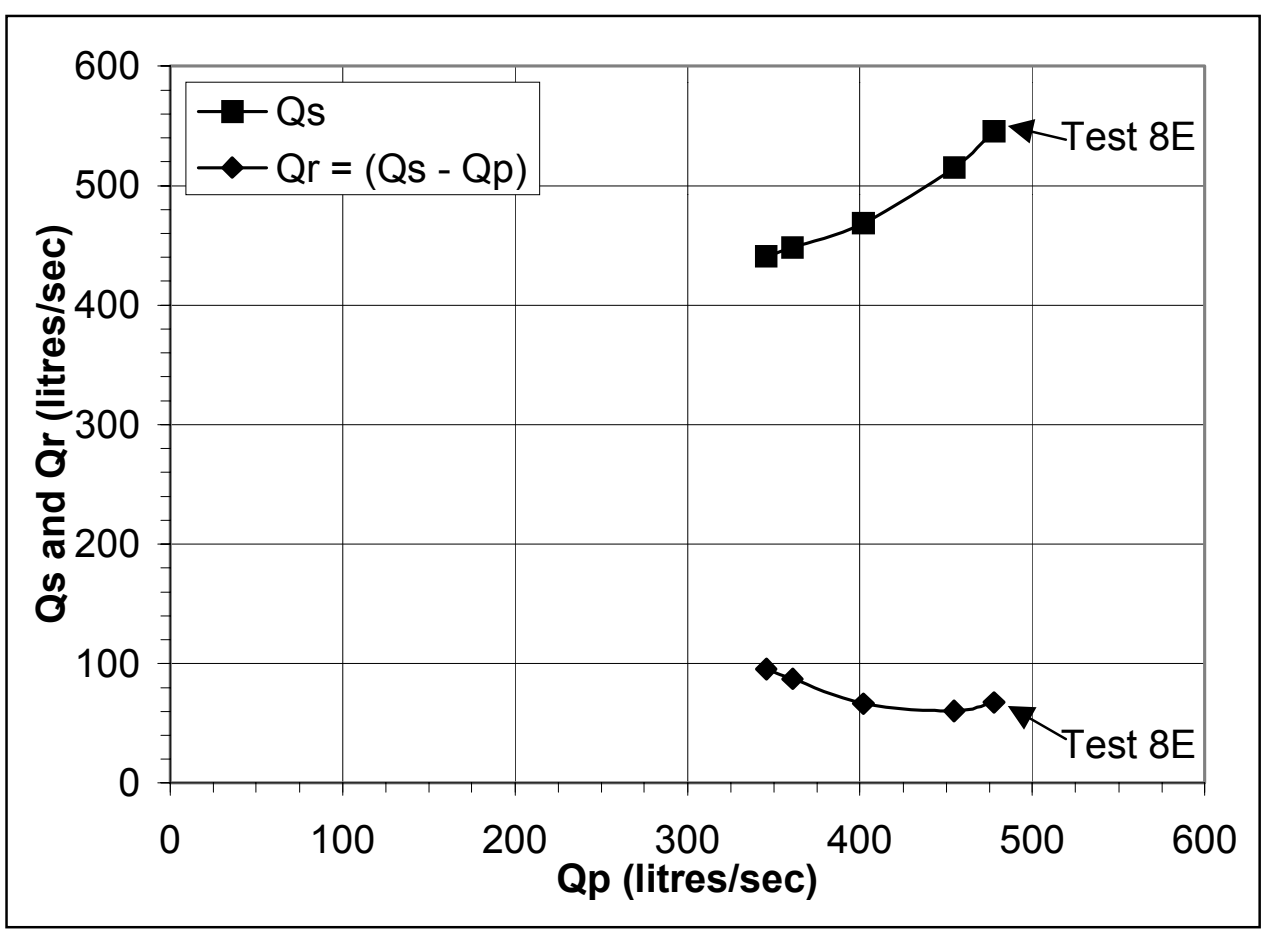

Figure 62. Surf zone and internal recirculation flow rates at Y27 for five irregular wave experiments

\section{Verification using gradient in $\mathbf{Q}_{\mathbf{s}}$ concept}

The second method proposed by Visser (1991) is based on the following hypotheses: (a) if $\mathrm{Q}_{\mathrm{p}}<\mathrm{Q}_{\mathrm{pu}}, \mathrm{Q}_{\mathrm{s}}$ increases in the downstream direction; (b) if $\mathrm{Q}_{\mathrm{p}}=\mathrm{Q}_{\mathrm{pu}}, \mathrm{Q}_{\mathrm{s}}$ is essentially uniform in the longshore direction; and (c) if $\mathrm{Q}_{\mathrm{p}}>\mathrm{Q}_{\mathrm{pu}}$, $\mathrm{Q}_{\mathrm{s}}$ decreases in the downstream direction.

Figure 63 shows results from 3 of the 15 regular wave experiments. In Test $6 \mathrm{D}, \mathrm{Q}_{\mathrm{p}}$ was approximately 40 percent less than the value of $\mathrm{Q}_{\mathrm{p}}$ in Test $6 \mathrm{~N}$ (the proper value), and $\mathrm{Q}_{\mathrm{s}}$ increases in the downstream direction. Conversely, in Test $6 \mathrm{~J}, \mathrm{Q}_{\mathrm{p}}$ was approximately 47 percent greater than $\mathrm{Q}_{\mathrm{p}}$ in Test $6 \mathrm{~N}$, and as shown, $\mathrm{Q}_{\mathrm{s}}$ decreases in the downstream direction. In Test $6 \mathrm{~N}, \mathrm{Q}_{\mathrm{s}}$ is essentially uniform in the alongshore direction. These results give credence to the conclusion made previously that Test $6 \mathrm{~N}$ represents the proper longshore current distribution for the regular wave case. However, in the case of under- or over-pumping, the longshore gradient in $\mathrm{Q}_{\mathrm{s}}$ is relatively small. Therefore, significant care should be taken during the iterative process of selecting and converging on the proper longshore current distribution.

Results from two of the five irregular wave experiments are shown in Figure 64. In Test $8 \mathrm{~A}, \mathrm{Q}_{\mathrm{p}}$ was approximately 28 percent less than the value of $\mathrm{Q}_{\mathrm{p}}$ in Test $8 \mathrm{E}$, and $\mathrm{Q}_{\mathrm{s}}$ increases in the downstream direction. Results for Test $8 \mathrm{E}$ (judged to be the proper value of $Q_{p}$ ) show that $Q_{s}$ increases slightly in the downstream direction, which suggests that $Q_{p}$ may have been slightly smaller than $\mathrm{Q}_{\mathrm{pu}}$. However, this contradicts evidence shown in Figure 62, which 


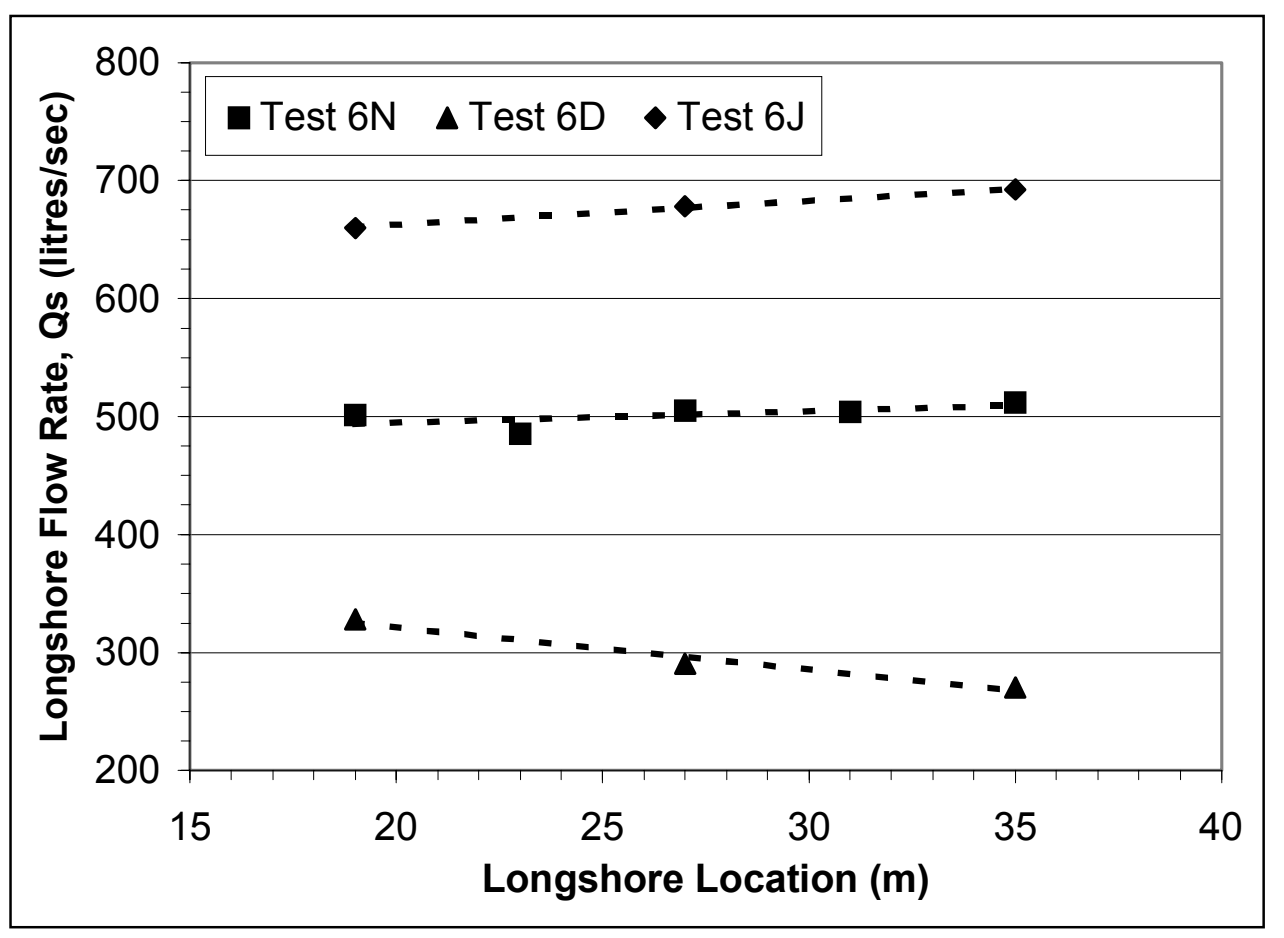

Figure 63. Regular wave test series: Longshore variation in surf zone flow rates, $Q_{S}$

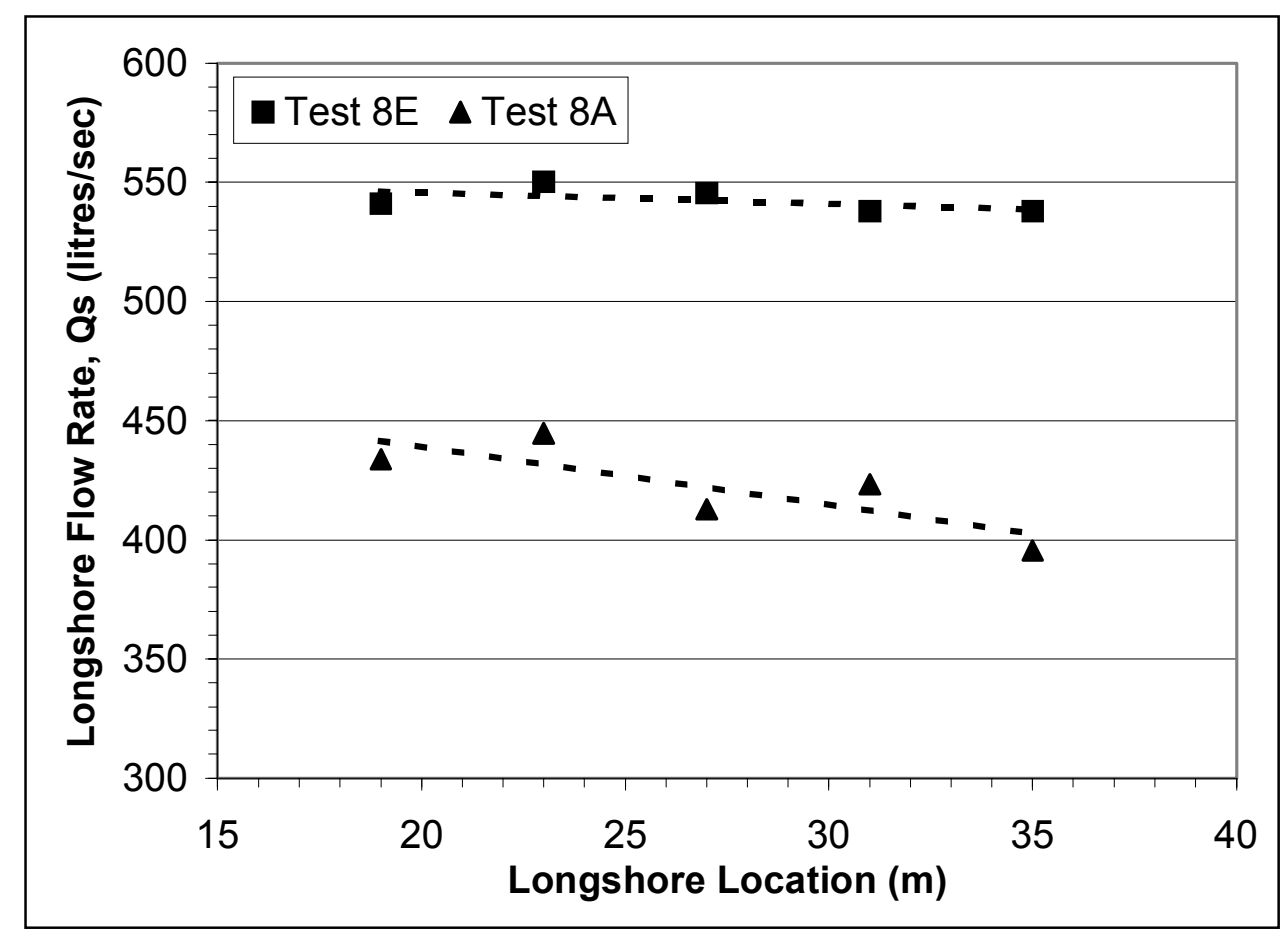

Figure 64. Irregular wave test series: Longshore variation in surf zone flow rates, $Q_{s}$ 
suggested that $\mathrm{Q}_{\mathrm{p}}$ was slightly greater than $\mathrm{Q}_{\mathrm{pu}}$. This slight discrepancy between the two methods may be caused by the fact that, in the LSTF, $Q_{p}$ can vary by as much as \pm 20 percent of $Q_{p u}$ without a significant increase in $Q_{r}$, as mentioned previously. Therefore, it was concluded that $\mathrm{Q}_{\mathrm{p}}$ in Test $8 \mathrm{E}$ was essentially the proper longshore current distribution for the irregular wave test series. A case involving significant over-pumping was not conducted for the irregular wave experiments.

\section{Longshore Uniformity}

This section quantifies the length of surf zone with the highest degree of longshore uniformity of the hydrodynamic processes. In general, it can be assumed that longshore uniformity should increase with increasing distance from the lateral boundaries. However, from the perspective of measuring longshore sediment transport in the LSTF, it is important to quantify the spatial limits of this region; especially at the downstream end where sand traps will be located.

Longshore uniformity was quantified by an average value of the standard deviation at each cross-shore position and at each transect within the length of surf zone being evaluated. For both Test $6 \mathrm{~N}$ and Test $8 \mathrm{E}$, the standard deviation was calculated independently for the wave height, mean water surface elevation, and mean longshore current data sets. A new value of the average standard deviation was calculated each time the representative beach length was decreased, by excluding data from one or more transects from the calculation. Transects at the upstream end of the facility were eliminated first, then transects at the downstream end were eliminated. The length of the surf zone with the highest degree of longshore uniformity is defined as the length at which a minimum standard deviation is obtained.

Figure 65 shows results for the regular wave experiment. Longshore variations in the average wave height measurements tend to decrease only slightly as the length of beach being considered is decreased. In contrast, longshore variations in the mean water surface elevation, and more importantly, in the mean longshore current, decrease significantly with decreasing length of testing region and approach a minimum asymptote at approximately $12 \mathrm{~m}$. If a shorter length of surf zone is considered, there is no significant increase in uniformity. Therefore, it is concluded that the hydrodynamic measurements have reached a minimum longshore variability if the length of surf zone being considered is reduced to $12 \mathrm{~m}$, starting at Y19 and extending upstream to Y31.

The high degree of longshore uniformity in this portion of the surf zone is illustrated in Figure 66, a through c, which show the cross-shore distributions of measured wave height, mean water surface elevation, and mean longshore current, respectively, for transects Y19 through Y31. Figure 66a shows that the greatest longshore variation in the measured wave height occurred at and immediately offshore of the incipient breaker line. Wave breaking occurred immediately shoreward of Wave Gauge 6. Deviations from the longshore averaged wave height were as high as \pm 8 percent. This is a laboratory effect caused by generating regular waves in a wave basin with reflective boundaries. However, the longshore variation in wave height measured in front of each of the 


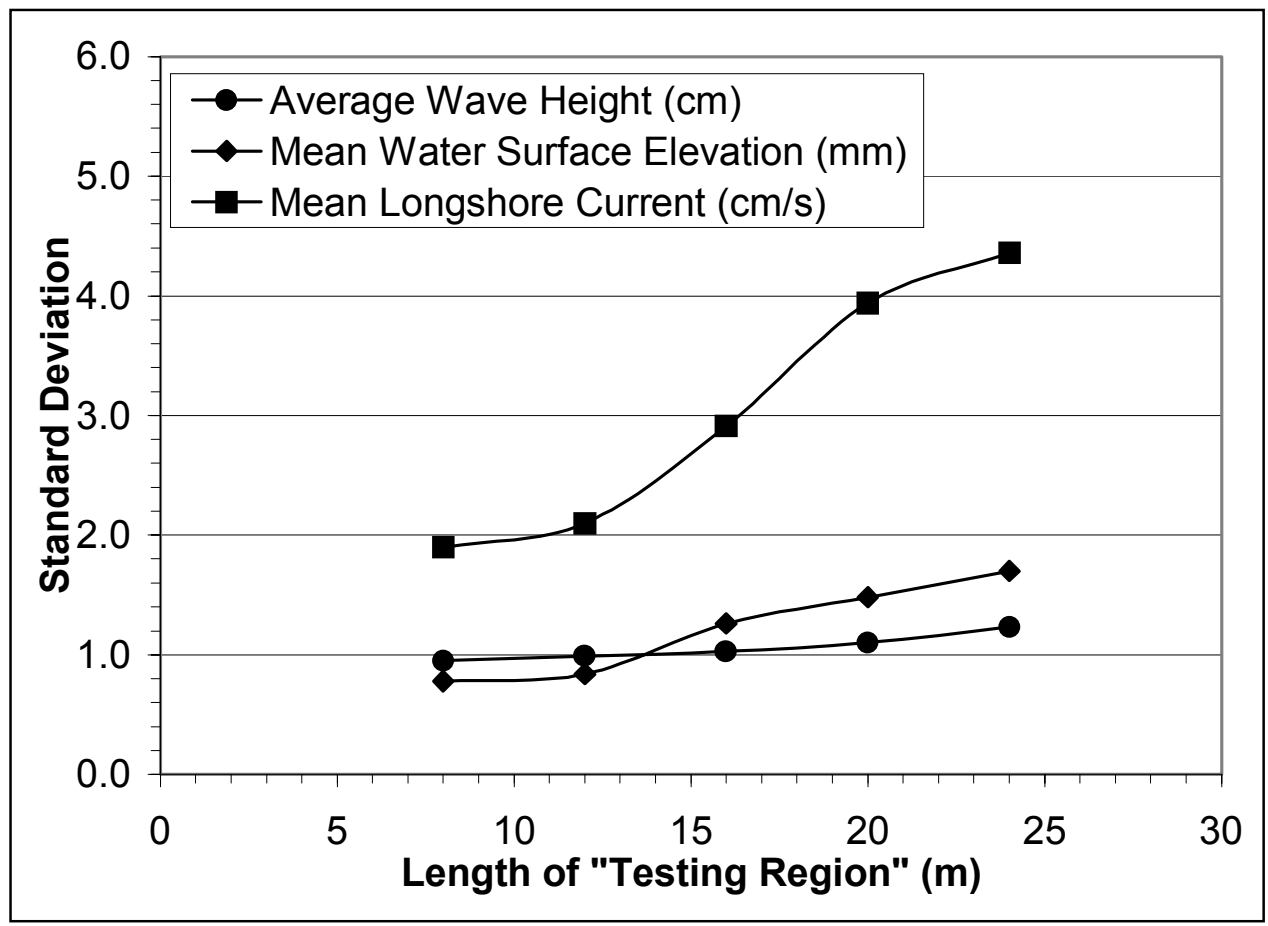

Figure 65. Regular wave Test $6 \mathrm{~N}$ : Longshore uniformity of hydrodynamics vs length of surf zone

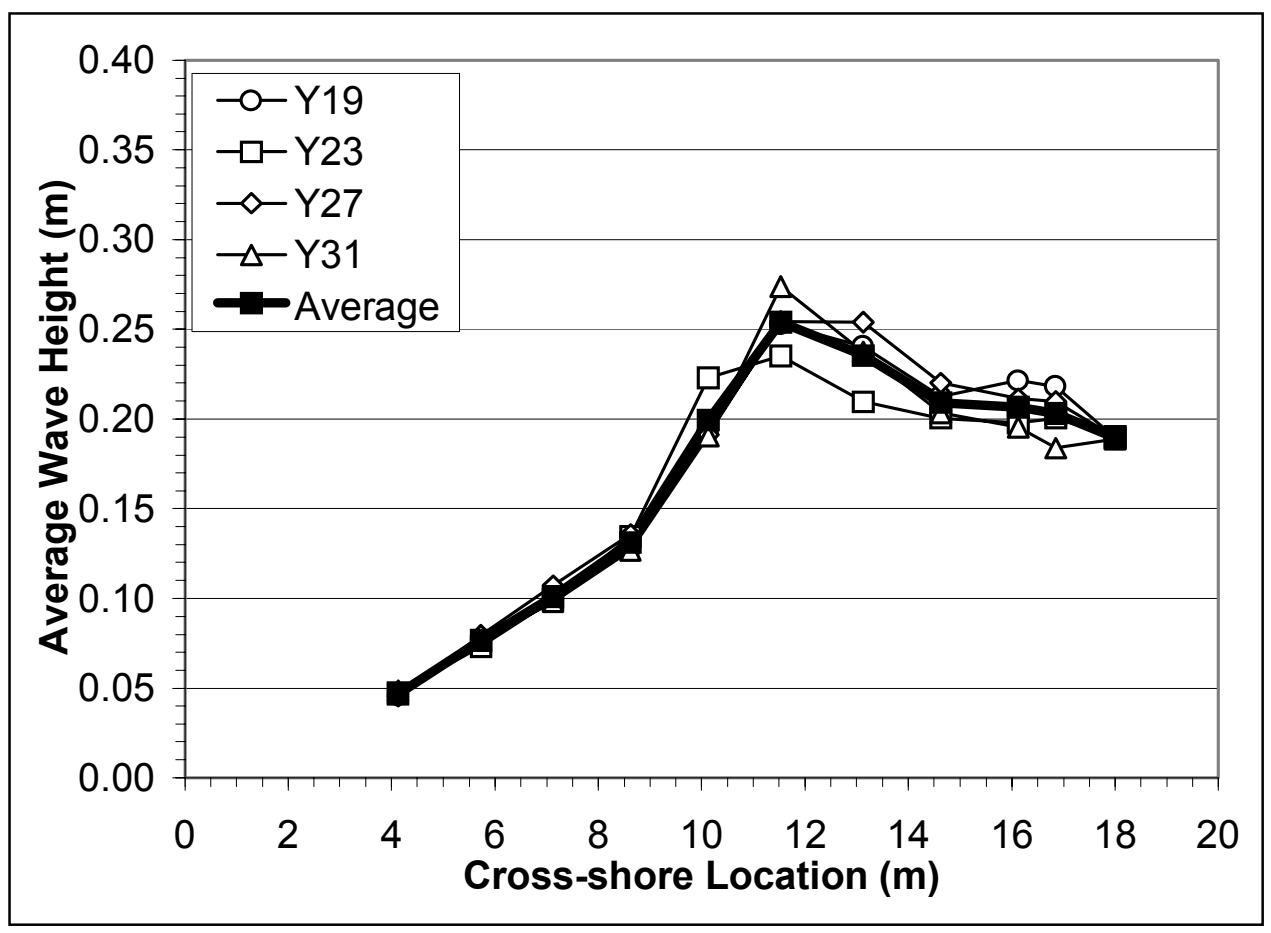

Figure 66. Longshore uniformity of surf zone

a. Regular wave Test $6 \mathrm{~N}$ : Cross-shore distribution of wave height (Part 1 of 3 ) 


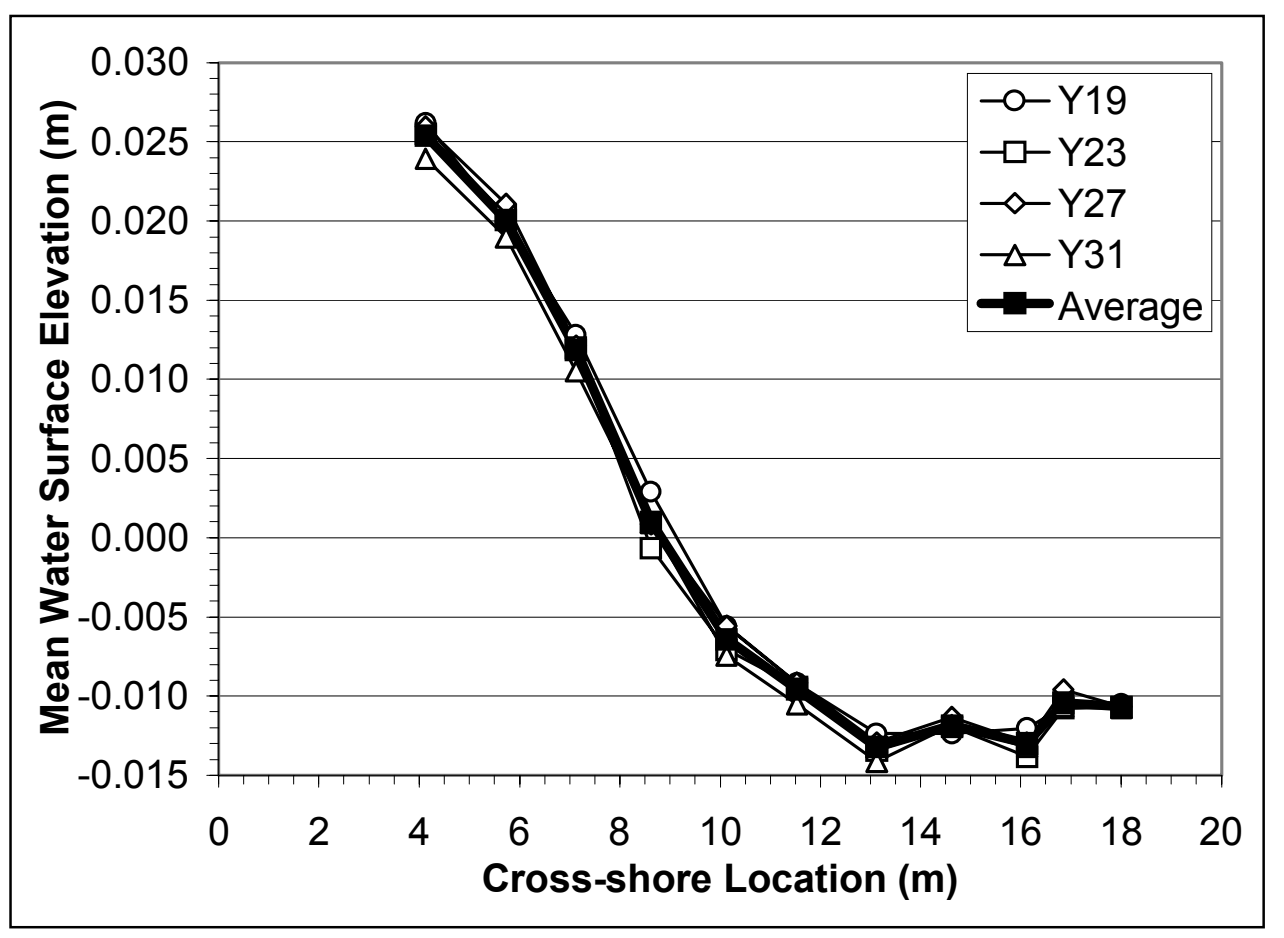

Figure 66. b. Regular wave Test $6 \mathrm{~N}$ : Cross-shore distribution of mean water surface elevation (Part 2 of 3 )

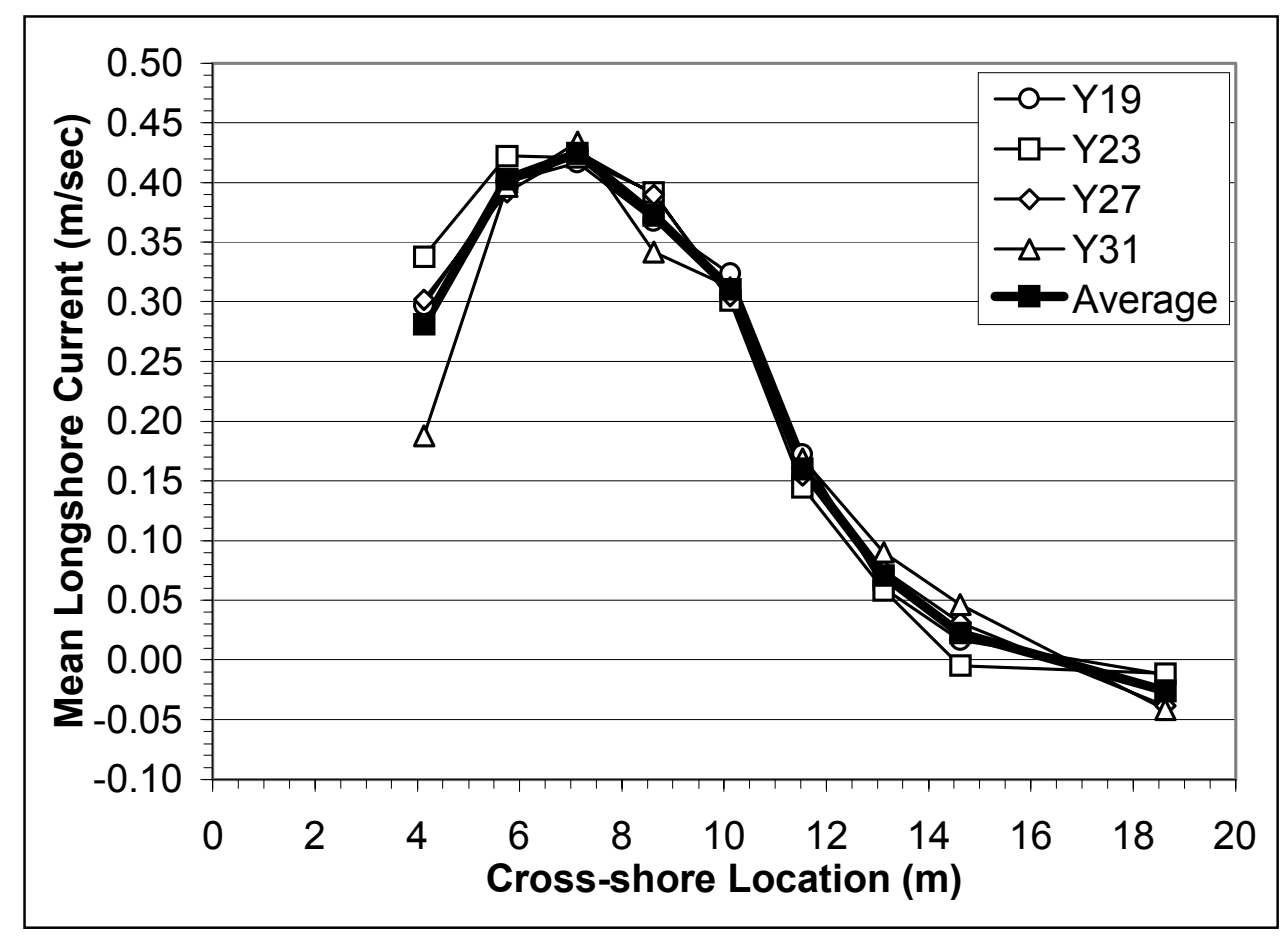

Figure 66. c. Regular wave Test $6 \mathrm{~N}$ : Cross-shore distribution of mean longshore current (Part 3 of 3 ) 
four generators $(x=18 \mathrm{~m})$, had a standard deviation of only 2.8 percent. In the inner surf zone, the longshore uniformity in wave height is very good due to the dominant effect of depth, which limits wave height. The longshore averaged breaker height index, across the width of the surf zone (Wave Gauge 1 through 6), is calculated to be 0.74 , and is tabulated in Table A-3, Appendix A, with several other parameters. Figure $66 \mathrm{~b}$ shows that the longshore variation in mean water surface elevation is approximately $\pm 0.0015 \mathrm{~m}$. This value is comparable to the elevation tolerance of the bridge support rails. Therefore, it can be concluded that there is no measurable longshore gradient in the mean water level in this region. Figure $66 \mathrm{c}$ shows that the degree of uniformity in the mean longshore current is quite good. The reduction in magnitude of the longshore current at $\mathrm{x}=$ $4.1 \mathrm{~m}$ at transect Y31 is caused by the small flow-reversal region farther upstream, near the shoreline. It is interesting to note that just offshore of the peak longshore current, the measurements suggest a slight flattening of the longshore current distribution. This observation is qualitatively consistent with the present understanding of the interaction of the undertow with the longshore current; see Putrevu and Svendsen (1992).

Figure 67 quantifies longshore uniformity of the hydrodynamic processes in the irregular wave experiment. As was found for the regular wave case, all three hydrodynamic parameters tend to approach a minimum asymptote, if the length of the testing region is reduced to approximately $12 \mathrm{~m}$, starting at Y19 and extending upstream to Y31. The values of the standard deviation in the irregular wave experiment are significantly less than in the regular wave experiment, especially for the wave and current data. Perhaps the regular wave forcing generates a basin response that does not occur when using irregular wave forcing.

Figure 68, a through c, illustrates the high degree of longshore hydrodynamic uniformity for the irregular wave case. Figure 68 a shows that the significant wave height is very uniform in the alongshore direction. The significant wave height at incipient breaking occurred immediately shoreward of Wave Gauge 7, based on visual observations and the fact that the gradient in the significant wave height curve increases significantly at that location. The longshore averaged breaker height index, across the width of the surf zone (Wave Gauges 1 through 7 ) is calculated to be 0.75 , as tabulated in Table A-3, Appendix A. The longshore averaged value of the measured maximum wave height also is shown to illustrate that $\mathrm{H}_{\max }>0.35 \mathrm{~m}$ at $\mathrm{x}=18 \mathrm{~m}$. As was the case for the regular wave test, Figure $68 \mathrm{~b}$ shows that the alongshore variation in mean water surface elevation is approximately $\pm 0.0015 \mathrm{~m}$. The wave setup at $\mathrm{x}=4.1 \mathrm{~m}$ is only about 60 percent of the value measured in the regular wave case, even though the incident wave energy was constant by setting $\mathrm{H}_{\mathrm{rms}}$ in the irregular wave case equal to $\mathrm{H}_{\text {reg }}$ in the regular wave case. Figure $68 \mathrm{c}$ shows that the mean longshore current is very uniform in the longshore direction. The peak current is $0.34 \mathrm{~m} / \mathrm{s}$, relative to a peak current of $0.42 \mathrm{~m} / \mathrm{s}$ in the regular wave case. The cross-shore distribution is broader than in the regular wave case, with the offshore tail decreasing much more uniformly. Dye was used to investigate the longshore current in very shallow water. No local increase in longshore current was detected shoreward of ADV 1 for either the regular or irregular wave case. It is interesting to note that the total longshore flow rate actively pumped through the lateral boundaries, $Q_{p}$ is 465 and $478 \mathrm{l} / \mathrm{sec}$ for the regular and irregular wave cases, respectively. These values are very similar, since the incident wave energy was held constant for the two cases, as mentioned previously. 


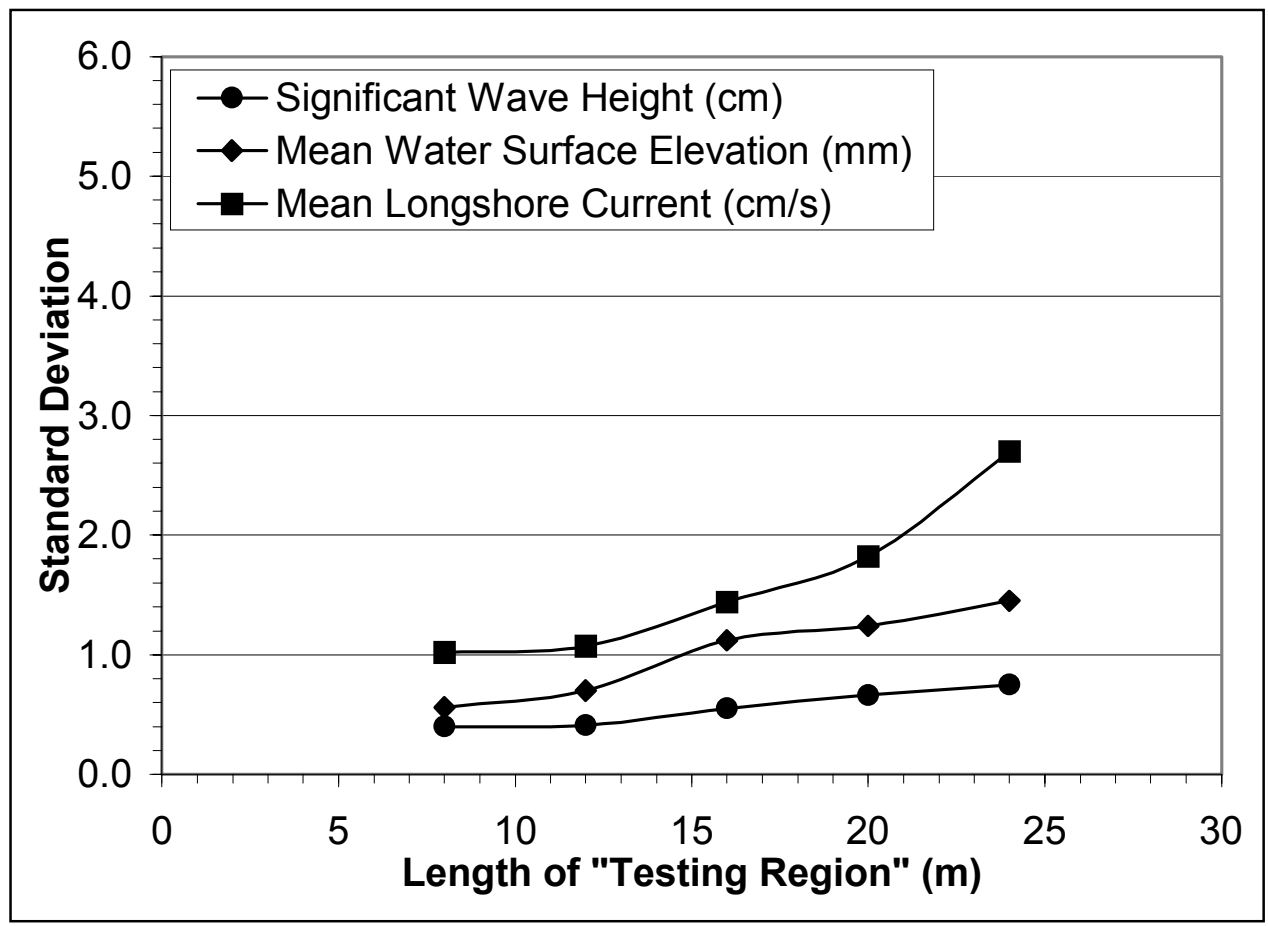

Figure 67. Irregular wave Test 8E: Longshore uniformity of hydrodynamics vs length of surf zone

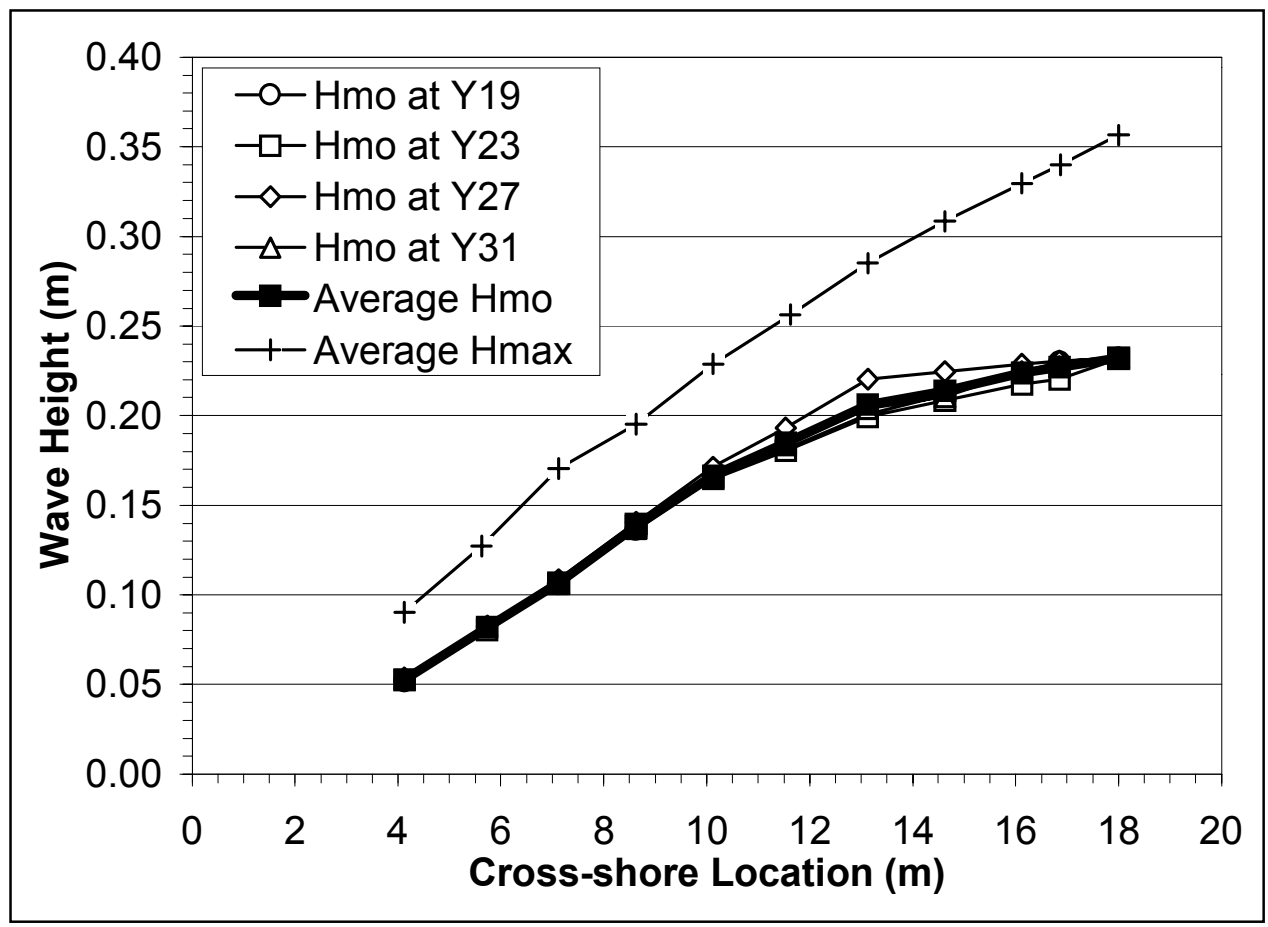

Figure 68. High degree of longshore hydrodynamic uniformity for irregular wave case

a. Irregular wave Test $8 \mathrm{E}$ : Cross-shore distribution of wave height (Part 1 of 3 ) 


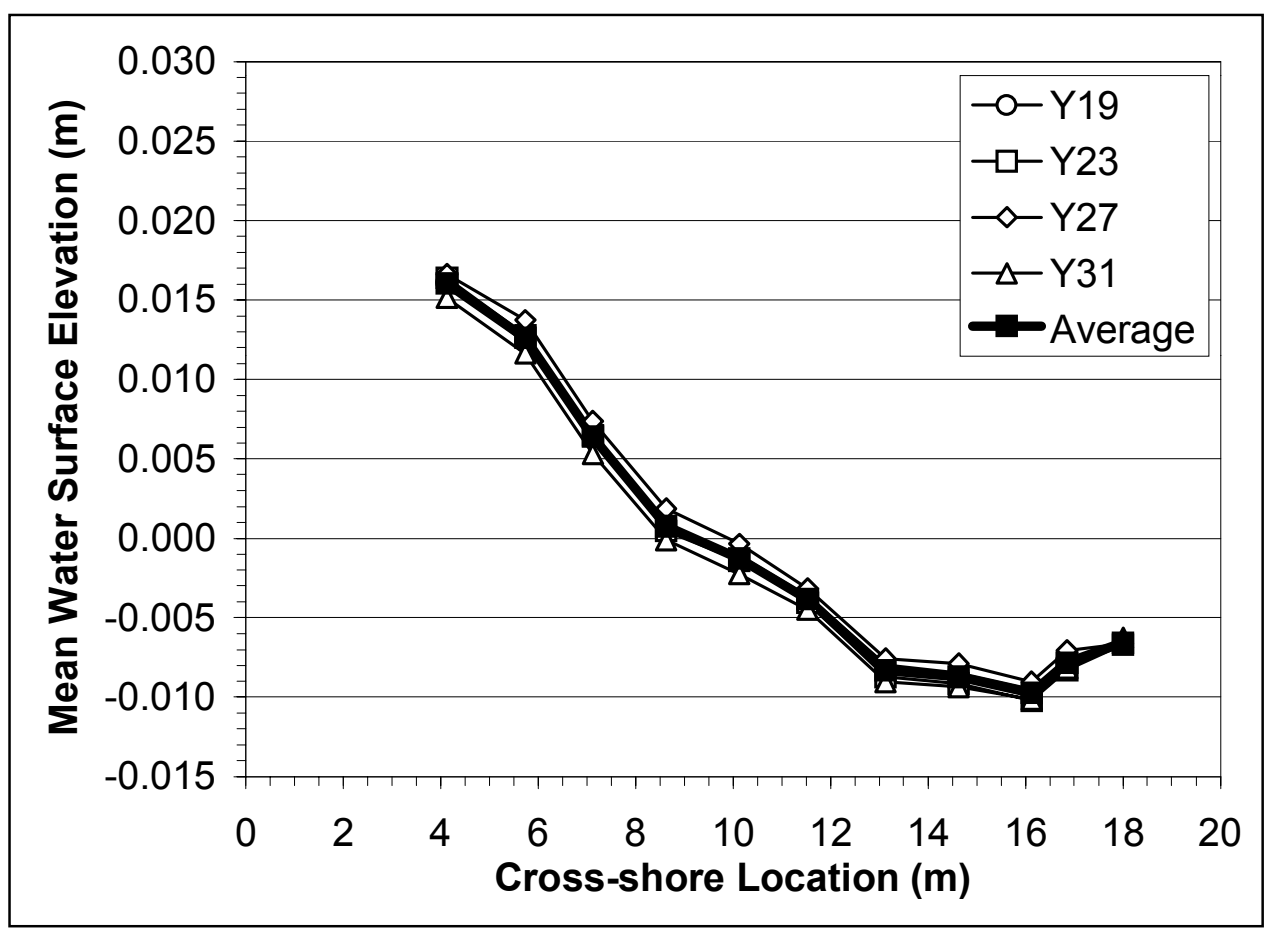

Figure 68. b. Irregular wave Test 8E: Cross-shore distribution of mean water surface elevation (Part 2 of 3 )

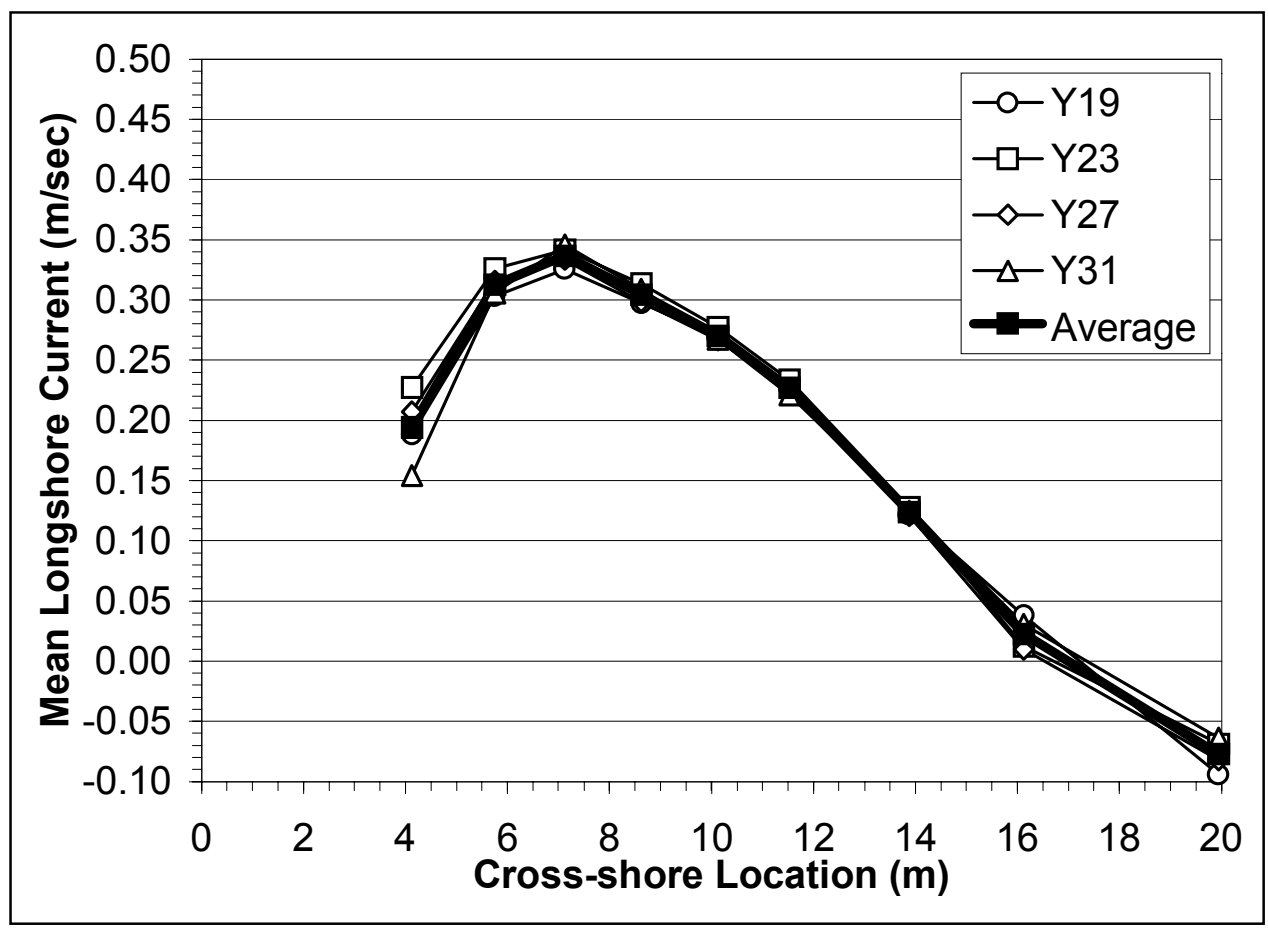

Figure 68. c. Irregular wave Test 8E: Cross-shore distribution of mean longshore current (Part 3 of 3 ) 
The measured cross-shore distribution of the mean longshore current at transects Y19 through Y31, and the longshore-averaged values of the mean longshore current for these four transects, are provided in Appendix A. The longshore averaged values of wave height and mean water surface elevation, from Y19 through Y31, are also provided.

\section{Longshore Current Steadiness and Repeatability}

Individual fixed-bed hydrodynamic experiments lasted from 2 to $3 \mathrm{hr}$, depending on the number of transects measured. Moveable-bed longshore sediment transport experiments also will be conducted continuously for a severalhour duration, perhaps longer. Therefore, the issues of current steadiness and repeatability over this time scale are important. Experiments were conducted to investigate the time required for the mean currents in the wave basin to reach steady-state conditions. Results from Test $8 \mathrm{E}$, the irregular wave case, are shown in Figure 69. The data represent the mean longshore current distribution measured at transect Y27 at three different times: 20, 110, and 150 min after the start of the experiment. Pumps were started at time zero. Wave generation commenced at the 10-min mark, after the 20 pumps had been adjusted to within 1 percent of the target discharge rates. Results showed that mean currents reached steady state within 10 min of starting the wave generators. The standard deviation of the mean longshore current, averaged for all cross-shore positions along Y27, was $0.0022 \mathrm{~m} / \mathrm{sec}$ and $0.0017 \mathrm{~m} / \mathrm{sec}$ for Test $8 \mathrm{E}$ and Test $6 \mathrm{~N}$, respectively.

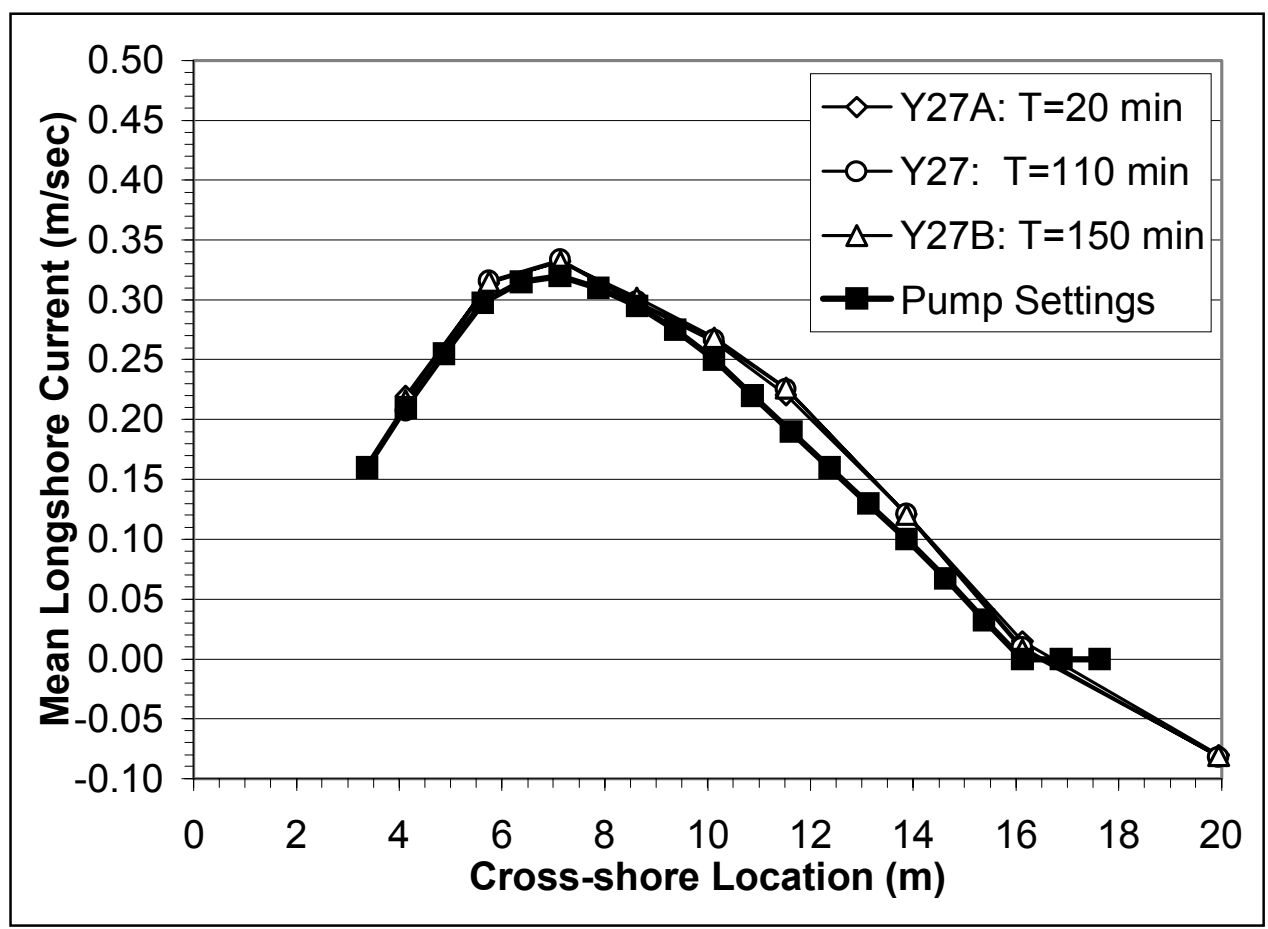

Figure 69. Irregular wave Test $8 \mathrm{E}$ : Sequential measurements of mean longshore current at $Y=27 \mathrm{~m}$ 
A related issue is measurement repeatability. Current measurements at Y27 were repeated five times during Test $6 \mathrm{~N}$, one immediately after the other, to quantify the repeatability of the mean longshore current measurements. Each set of measurements was sampled for $500 \mathrm{sec}$. The standard deviation of the five mean longshore current measurements, averaged for all cross-shore positions, was $0.0015 \mathrm{~m} / \mathrm{sec}$. This confirmed that the repeatability of ADV measurements was quite good.

The steadiness of the current regime and the repeatability of the ADV measurements allowed the vertical mean current structure to be measured with a high degree of confidence. Measurements were made by accurately repositioning all of the ADV sensors at a new elevation in the water column, prior to each subsequent set of measurements. The vertical structure of the mean longshore current for Test $6 \mathrm{~N}$ and Test $8 \mathrm{E}$ are given in Figures $\mathrm{A}-1$ and $\mathrm{A}-2$, Appendix A.

\section{Application to Sediment Transport Experiments}

As discussed in the previous section, the mean currents in the wave basin reached steady state within $10 \mathrm{~min}$ of starting the wave generators. In addition, with experience, approximately five iterations are required to converge on the proper pump settings. These are two very positive conclusions with respect to future sediment transport experiments in the LSTF, because the time will be minimized during which sediment will be moving in response to improper longshore currents.

As shown, the high cross-shore resolution of the longshore current recirculation system in the LSTF allows very accurate control of the longshore current distribution. Because the pumps are digitally controlled, and the in-line flow sensors allow for real-time data collection, it typically only takes 30 to 60 minutes to readjust the pump settings for a new iteration. Therefore, during the sediment transport experiments, the pump settings can be easily adjusted in response to changing beach morphology.

Most of the longshore sediment transport experiments will be conducted using irregular waves. For the irregular wave case, the degree of longshore uniformity in mean longshore current and wave height is quite good downstream to Y14, the closest transect to the downstream boundary. As shown in Figure 70, there were small decreases in longshore current speed near the downstream boundary, on the order of 10 percent at the peak, relative to the average current for the 12-m region of the beach with the highest degree of longshore uniformity (Y19 through Y31). The reasonably high degree of longshore uniformity at the downstream end of the beach is a very positive result from the standpoint of conducting longshore sediment transport experiments in the LSTF, since the sand traps will be located at the downstream end of the beach. Nonetheless, some inefficiency is expected at the sand traps because of the slight reduction in current magnitude and wave energy at the downstream boundary. 


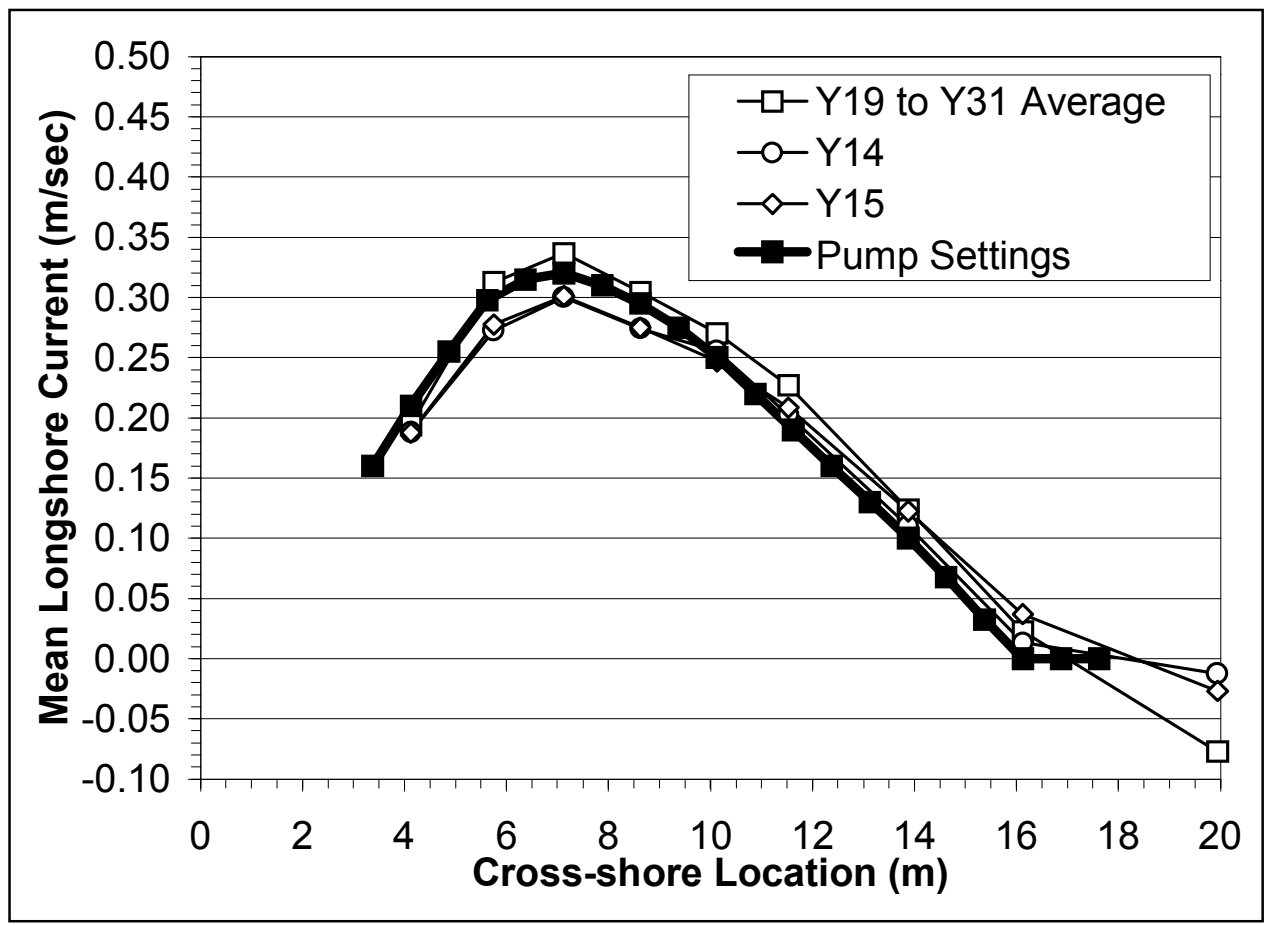

Figure 70. Irregular wave Test 8E: Distribution of mean longshore current at downstream boundary 


\section{Longshore Sediment Transport Experiments}

\section{Introduction}

This chapter discusses the methods and procedures that were used to execute movable-bed longshore sand transport (LST) experiments. The objective of the experiments described here was to accurately measure the total LST rate, and its cross-shore distribution, on a laboratory beach that is intended to represent an infinitely long, natural beach having straight and parallel topographic contours, i.e., water depths are invariant in the alongshore direction. For these idealized beach conditions, along with wave and current forcing that has alongshore uniformity, the LST rate also should be invariant in the alongshore direction. Longshore uniformity of all processes represents the target conditions that are to be created in the facility. This chapter presents results from initial experiments performed in the facility, and provides information on how uniform the conditions were.

Ideally, the sediment traps situated at the downdrift end of the beach should be 100 percent efficient, i.e., completely trap the sand that the waves and currents transport alongshore, no more, no less. However, the physical boundaries of the facility, and imperfections in the systems and scheme used to control wave, current, and sediment transport processes at the lateral boundaries, influence the degree to which alongshore uniformity can be achieved. Chapter 9 addressed how well uniformity was achieved, in terms of hydrodynamics, for a planar concrete beach with straight and parallel contours. The situation becomes much more complex for movable-bed experiments. With a mobile bed, sand begins to immediately move in response to waves and currents and the beach topography begins to evolve. Imperfections in lateral boundaries are expected to induce anomalous beach response, loss of complete alongshore uniformity, and a lessthan-perfect trapping system. The degree of beach uniformity that is achieved in the alongshore direction, and maintained during the course of experiments, dictates the success of the experiments. The first few experiments were a learning process. During the initial experiments, methods and procedures for conducting movable bed experiments were developed and refined; and results and lessonslearned are discussed here.

Results from two experiments are presented in this chapter. In the first, predominantly spilling-type breaking waves were produced. These were created 
by specifying incident irregular waves that had a significant wave height at the wave generator of $0.25 \mathrm{~m}$ (in $0.9 \mathrm{~m}$ of water) and a peak spectral wave period of $1.5 \mathrm{sec}$. Incident waves for the second experiment were also irregular, but had a significant wave height of $0.23 \mathrm{~m}$ and a peak spectral period of $3.0 \mathrm{sec}$. These lower-steepness waves produced predominantly plunging-type wave breaking. The incident wave direction for both experiments was $10 \mathrm{deg}$, relative to shore normal, at the wave generator. These two wave cases represent the upper magnitude of incident wave energy, in terms of significant wave heights, for which LST experiments can be effectively performed in the facility.

\section{Issue of Sand Recharging}

As waves and currents begin to transport sand along the beach, a deficit of sand is created at the updrift boundary if no sand is introduced. Originally, a system was envisioned that would actively recirculate sand during an experiment, and introduce it at the updrift end of the beach in a prescribed cross-shore distribution that was to be determined from trap measurements made at the downdrift end. The system would recirculate a water-sand slurry in the same manner as the pumps recirculate water (Chapter 3 ) and continuously discharge it along the updrift boundary of the beach in a controlled manner. A sizable effort was expended on both conceptual and more detailed engineering designs of such a system. Both hydraulic and mechanical systems were considered for introducing sand at the upstream end, and several design concepts for each type of system were evaluated. However, in light of uncertainties associated with each design, it appeared that even though considerable resources could be spent on a sand-recharging system, performance of the system might not meet requirements and expectations.

Concurrent with work to design an automated recharge system, preliminary experiments were conducted with the mobile sand beach. Results suggested that alongshore uniformity over the downdrift two-thirds of the beach was reasonably well maintained during what was anticipated at the time to be a typical experiment duration for moderate to high LST conditions ( 1 to $4 \mathrm{hr}$ ). As anticipated, a beach discontinuity formed at the updrift end of the facility, but the erosion-induced perturbation did not rapidly propagate down the beach during a several-hour period, even under rather high longshore transport conditions (some of the largest that can be produced in the facility). Perturbations were mostly confined within a 5-m zone immediately adjacent to the updrift boundary. The duration of experiments is also limited by the capacity of the traps (Chapter 4). The total amount of sand that was transported alongshore during a several-hourlong experiment for high-transport conditions is generally less than 1 percent of the $125 \mathrm{~m}^{3}$ of sand that comprises the artificial beach $\left(D_{50}=0.15 \mathrm{~mm}\right)$.

After considering a number of factors, including technical difficulty and inherent limitations, time-to-construct, and monetary constraints, a decision was made not to proceed with construction of an active sand recharge system.

Instead, a system of dredging and recirculating sand off-line after an experiment was completed was adopted (Chapter 4). The system involves use of a trashpump to recirculate the sand-water slurry and positioning of the discharge point to roughly fill in the areas where the sand deficit is greatest. The purposes of the 
off-line beach replenishment are twofold: recharge the sediment supply at the updrift end of the beach, and restore the beach to one with straight and parallel contours. Following sand recirculation, the wave basin is drained, and remolding of the beach to the desired shape is accomplished (Chapter 5 for more details). The middle two-thirds of the beach generally requires little, if any, remolding due to the alongshore-uniform condition that is maintained in this region. Typically, there also are slight perturbations very close to the downdrift boundary, primarily accretion. Beach response at the downdrift end is discussed in more detail further into this chapter.

\section{Procedures for Executing Experiments}

\section{Test sequence}

For each experiment (an experiment is characterized by use of a singleincident wave condition), process measurements were made for a series of test segments, or wave runs. Each segment lasted from approximately 40 to $200 \mathrm{~min}$ in duration, depending on the quantity of sand that was transported along the beach. Shorter-duration tests were adopted for higher, longshore, transport conditions. Duration also was dictated by the purpose of the test segment. Each segment was designed to focus on one of the following progressive goals, with the final goal being acquisition of accurate and comprehensive measurements of the LST rate, and its cross-shore and vertical distribution patterns. The progressive goals, listed in sequential order, include:

a. Obtain optimal settings for the pump circulation system, i.e., pump the proper longshore current, to minimize boundary influences and minimize recirculation of the longshore current.

$b$. Allow the beach to reach equilibrium, or near-equilibrium, conditions in terms of beach profile shape.

c. Provide adequate measurement coverage in the alongshore and crossshore directions.

d. Provide adequate measurement coverage throughout the water column.

$e$. Repeat key measurements to ensure data quality and repeatability.

Each individual test segment followed a set procedure to ensure data comparability. The procedure adopted for each test segment was as follows:

a. Conduct a complete pretest beach survey.

$b$. Perform pretest sediment trap sampling (under quiescent conditions to "zero" the traps).

c. Check instruments, data acquisition systems, and initialize sensors.

d. Start sampling of sediment trap weight.

e. Start longshore current circulation system (sequential start-up of pumps, working onshore to offshore).

$f$. Start wave generators (synchronous start-up of all four).

$g$. Begin sampling of wave, current, and sediment concentration.

$h$. Stop the wave generators.

$i$. Stop the longshore current circulation system. 
$j$. Perform sampling of final trap weights (under quiescent conditions).

$k$. Wash sand from the traps' rubber seals into the traps and resample weights.

l. Conduct a complete posttest beach survey.

\section{Instrumentation and measurement scheme}

The LSTF hosts a suite of instrumentation. Details on the capabilities and accuracy of the instruments are described in previous chapters (see Chapters 2 and 8). A brief summary of the instrumentation and sampling scheme specific to the LST experiments is listed in Table 5.

\begin{tabular}{|c|c|c|c|c|c|}
\hline $\begin{array}{l}\text { Parameter to } \\
\text { be Measured }\end{array}$ & $\begin{array}{l}\text { Instrument } \\
\text { Type } \\
\end{array}$ & Sampling Rate & $\begin{array}{l}\text { Sampling } \\
\text { Duration }\end{array}$ & $\begin{array}{l}\text { Number } \\
\text { of Cross- } \\
\text { shore } \\
\text { Locations } \\
\end{array}$ & $\begin{array}{l}\text { Vertical } \\
\text { Profile } \\
\end{array}$ \\
\hline Waves & $\begin{array}{l}\text { Capacitance } \\
\text { Wave Sensor }\end{array}$ & $20 \mathrm{~Hz}$ & $10 \mathrm{~min}$ & $10^{1}$ & $\mathrm{~N} / \mathrm{A}$ \\
\hline Current & $\begin{array}{l}\text { Acoustic Doppler } \\
\text { Velocimeter } \\
\text { (ADV) }\end{array}$ & $20 \mathrm{~Hz}$ & $10 \mathrm{~min}$ & $10^{2}$ & Yes \\
\hline $\begin{array}{l}\text { Sediment } \\
\text { Concentration }\end{array}$ & $\begin{array}{l}\text { Fiber-Optic } \\
\text { Backscatter } \\
\text { (FOBS) }\end{array}$ & $16 \mathrm{~Hz}$ & $10 \mathrm{~min}$ & 7 & Yes \\
\hline Water Depth & $\begin{array}{l}\text { Bottom-tracking } \\
\text { Profiler }\end{array}$ & Every $5 \mathrm{~mm}$ & Continuous & 3,660 & $N / A$ \\
\hline Sediment Flux & $\begin{array}{l}\text { Bottom } \\
\text { Sediment Traps }\end{array}$ & $\begin{array}{l}\text { Mode 1: } 4 \mathrm{~Hz} \\
\text { Mode 2: } 1 \mathrm{~Hz}\end{array}$ & $\begin{array}{l}\text { Continuous } \\
1 \mathrm{~min} \\
\end{array}$ & $\begin{array}{l}20 \\
20 \\
\end{array}$ & $\begin{array}{l}\text { No } \\
\text { No }\end{array}$ \\
\hline
\end{tabular}

Sediment flux measurements using the downdrift bottom traps were conducted in two modes. Mode 1 consisted of continuous sampling of trap weight at a high frequency, $4 \mathrm{~Hz}$, during the test segment. However, accurate weight measurements during the wave run were not possible due to vibration and movement of the traps, which were forced by the wave motion. Measured weights exhibited considerable "noise," but increasing trends were identifiable. Mode 2 trap measurements consisted of two discrete sampling periods, at $1 \mathrm{~Hz}$, before and after the wave run. Accurate trap weights were obtained in quiescent water.

Wave and current measurements were synchronized, allowing computation of directional wave spectra. However, estimation of breaking wave angle from the spectra was difficult, and calculations had a high degree of uncertainty. Breaker angle also was measured visually using the digital compass in an electronic total station transit. About 20 breaker angles were measured during each test segment. An overall average for all the test segments for each wave condition was used to estimate the breaker angle. Elevations of the FOBS sensors are referenced to the bottom sensor, which is deployed directly on the sand bed. The bottom sensor is identified as the one having saturated signals. 


\section{Complexities of pumping the proper longshore current}

The process of determining proper pump settings for movable-bed LST experiments is much more complex than that for experiments involving a concrete beach. In the latter case, the bottom does not respond during the iterative process in which incorrect pump settings are gradually improved to converge on the proper settings. The iterative process on a concrete beach is much more "forgiving," unlike that for movable-bed experiments, in which the bed immediately begins to respond to wave forcing and incorrect pump settings. The recommended iterative procedure involves approaching the proper settings from below. In this situation, the discharge pumped is less than the proper amount, and return flows tend to transport sand offshore along the downdrift boundary. This creates an undesirable beach response that is nonuniform in the alongshore direction and occurs directly in front of the traps, which in turn alters wave breaking and current patterns near the downdrift end of the beach. Also, during each iteration, measurements must be made at multiple transects to assess the degree of alongshore uniformity that has been achieved. More transects (i.e., more data) are desirable to ascertain the degree of alongshore uniformity that exists. But more time is required to make them, and during this time the beach is responding to improper flow conditions. Typically the process to converge on the proper pump settings will require from 8 to $12 \mathrm{hr}$ of run time. Depending on the longshore transport rate, this may require remolding of the beach anywhere from one to three times. Beach remolding is a very time-consuming process, and one to be minimized.

\section{Equilibration of beach profile shape}

Evolution of the beach to a near-equilibrium profile shape also requires time. The time frame to reach a near-equilibrium beach profile condition depends on the degree to which the preexperiment profile is out of equilibrium with the incident wave conditions and the rate of sediment transport. The goals of arriving at an equilibrium beach profile shape and of establishing the proper pump settings are accomplished concurrently. Usually, the beach must be remolded several times during this process.

Figure 71 illustrates profile evolution for the irregular wave case that produced plunging-type breakers. Profile measurements were made near the center of the beach. A number of profiles are shown. The different curves shown in the figure correspond to different elapsed times relative to the starting time. Included in the figure are the pretest profile, profiles measured at the conclusion of numerous test runs that varied in duration from 40 to $100 \mathrm{~min}$, profiles measured before and after beach rebuilding, and the profile shape after $10.5 \mathrm{hr}(630 \mathrm{~min})$ of wave activity. After approximately $180 \mathrm{~min}$, the beach had evolved to its near-equilibrium state for these incident wave conditions. 


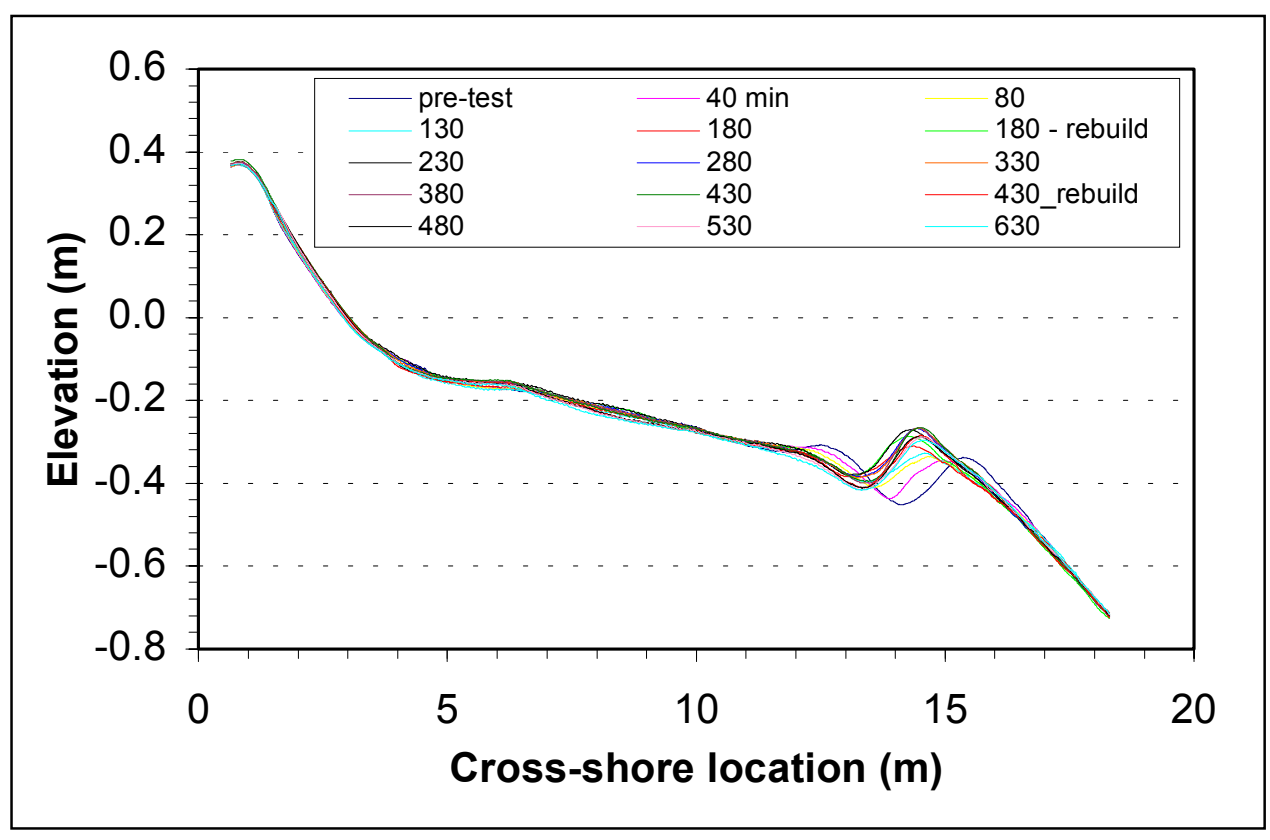

Figure 71. Evolution of beach profile shape for the plunging-breaker case

Note that the pretest profile had a break-point bar that is approximately $1 \mathrm{~m}$ seaward of, and in deeper water than, the bar on the near-equilibrium profile. A larger incident wave height was used initially in the plunging-breaker experiment. However, the location of incipient wave breaking was too close to the offshore-most flow channels. Therefore, a decision was made to reduce the incident wave height. The position and shape of the bar evolved fairly rapidly in response to the change in wave height, reaching the near-equilibrium condition after only a few hours. The inner portions of the profile changed relatively little, compared to the dramatic changes observed in the bar region. The profile shape that was molded initially, for the plunging-breaker experiment, was the equilibrium profile shape that was produced by the irregular waves that had a similar incident significant wave height but were characterized by spilling breakers.

The beach profile shape for the spilling breaker case evolved to its nearequilibrium condition more slowly, taking approximately $14 \mathrm{hr}$. The difference is attributed to the fact that the initial profile for this case was molded to a theoretical equilibrium profile shape, approximated by a series of planar beach sections. Evolution to equilibrium required much more movement of sand from the inner- and midsurf zone to the offshore. Evolution to equilibrium for the plunging-breaker case primarily involved sand movement in the bar region.

\section{Steadiness and repeatability of measurements}

Process measurements were made for 10-min sampling intervals. The instrumentation bridge was stationary at one alongshore coordinate during each 10-min interval. To obtain measurements at other cross-shore transects, the bridge was repositioned and measurements were acquired for another 10-min 
interval. The number and location of transects can be programmed in advance, repositioning of the bridge can be handled automatically, and movement from one location to another is done rather quickly. Acquisition of data at five transects in this manner takes approximately $1 \mathrm{hr}$.

Four FOBS arrays were available, which occasionally required repositioning. To obtain sediment concentration data at a denser spatial resolution in the crossshore direction, sensor arrays had to be manually repositioned along the bridge, and 10-min test segments had to be repeated. Also, since the current sensors measure velocity at only one elevation in the water column, repositioning of the sensors was required to make measurements for characterizing the vertical current structure. Measurements at other elevations required manual adjustment of the sensor elevation. Manual repositioning of sensors increased the amount of time needed to make measurements. Approximately 2 to $2.5 \mathrm{hr}$ were required to obtain velocity measurements at eight or nine positions throughout the water column, at one alongshore location.

Making measurements over this length of time raises questions concerning measurement repeatability and steadiness of hydrodynamic conditions. In Chapter 9, measurements made during fixed-bed concrete beach experiments, which lasted for several hours, showed that conditions were steady during the experiment duration. Figure 72 shows the mean longshore current measured at a number of cross-shore locations at transect Y22 (near the center of the beach at the alongshore coordinate of $22 \mathrm{~m}$ ). Measurements were made at the beginning (Y22a), middle (Y22b), and end (Y22c) of an approximately 2-hr-long wave run designed to acquire a high alongshore density of cross-shore transects and to examine steadiness of the mean longshore current for the spilling breaker case. The curve labeled "LB's" in the legend refers to the lateral boundary conditions, i.e. the pumped discharges converted to current speed. Results show that for the movable-bed case, steadiness in mean longshore current is good. Gaps in the measured data reflect sensor malfunctions.

The issue of measurement repeatability is related but different, in the sense that steadiness refers to conditions within a single wave run or test segment, whereas, repeatability refers to variations from test to test for the same beach, wave, and current conditions. To examine repeatability, measurements were made at the same transect and then compared for a number of wave runs in which a near-equilibrium beach condition existed, and wave/current forcing was held constant. Figures 73 and 74 show measured significant wave height for both the spilling- and plunging-breaker wave cases, respectively. Measurements were made near the center of the beach (at alongshore coordinates of $22 \mathrm{~m}$ for the spilling case, and $24 \mathrm{~m}$ for the plunging case). The beach was at its nearequilibrium profile shape in both instances. Repeatability in wave measurements is good from run to run. 


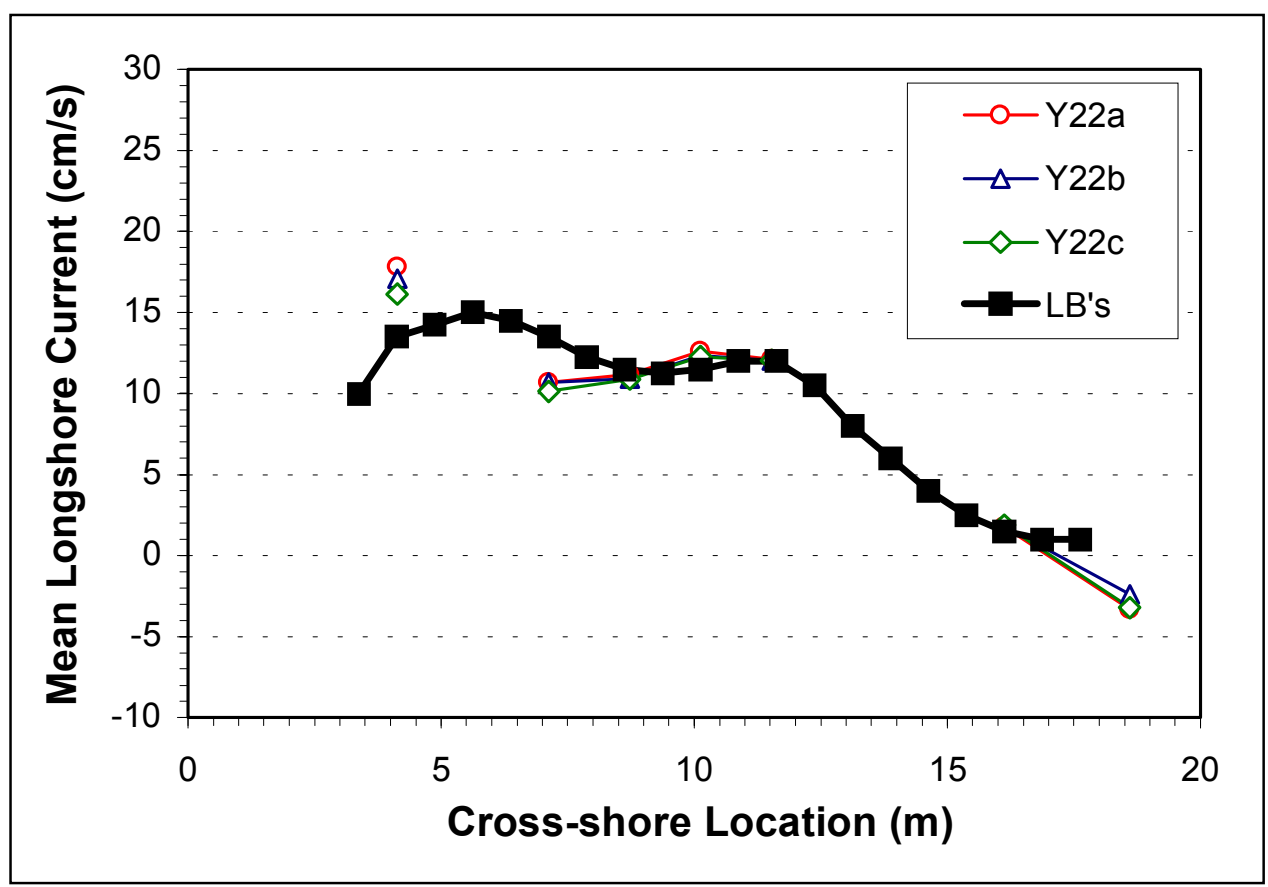

Figure 72. Repetitive measurements of mean longshore current during a singlewave run

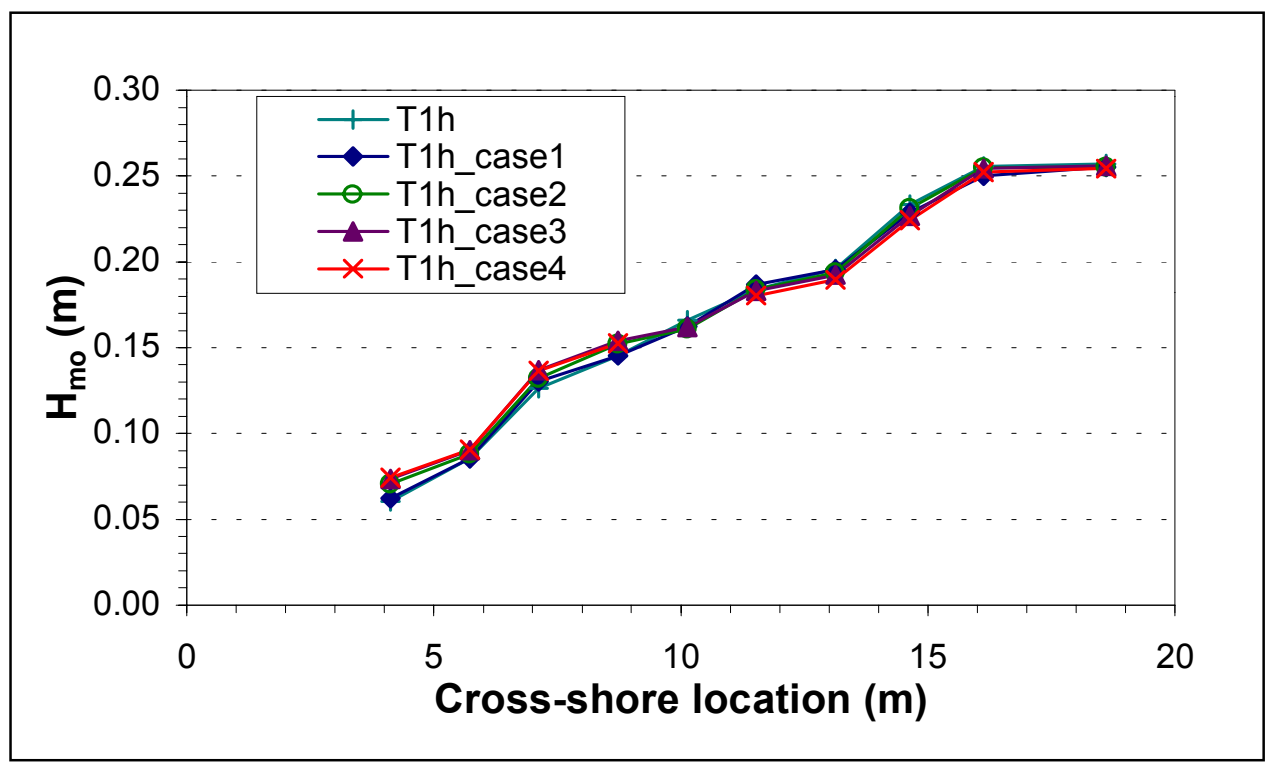

Figure 73. Measurements of significant wave height for successive wave runs at the same cross-shore transect for the spilling-breaker case 


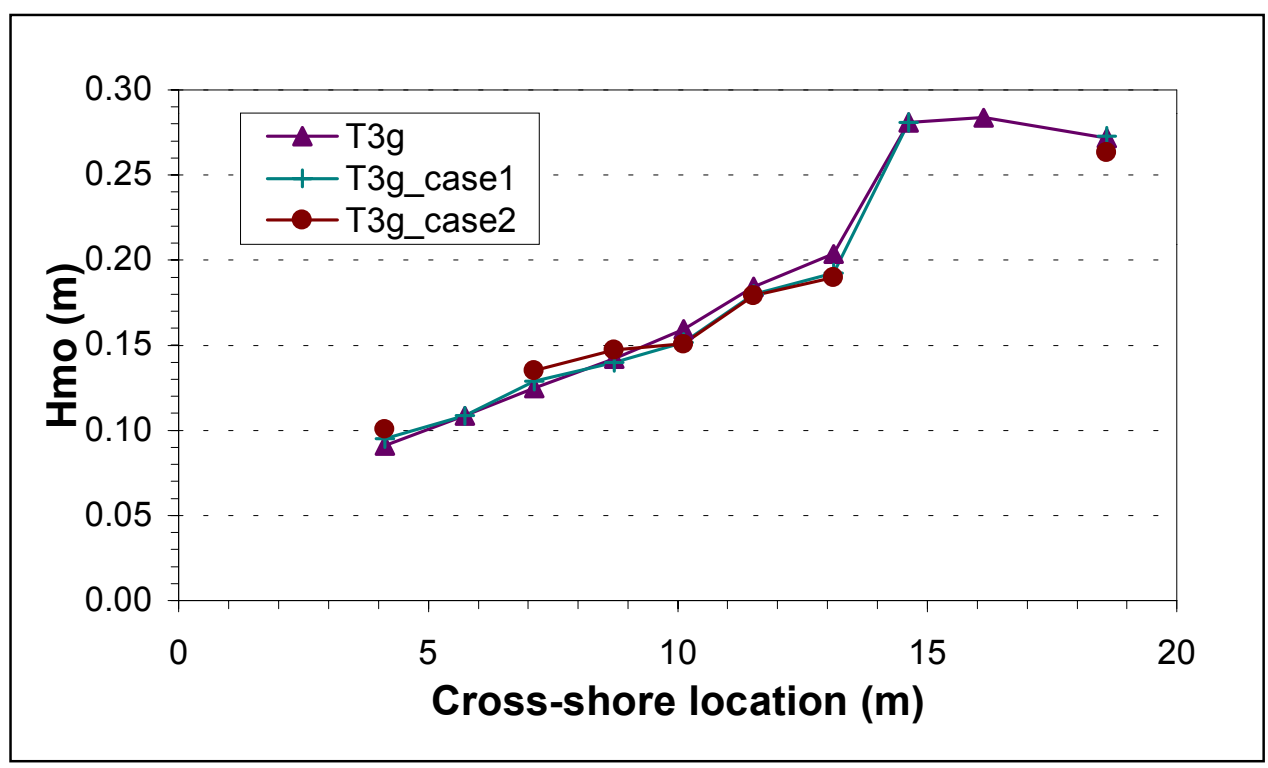

Figure 74. Measurements of significant wave height for successive wave runs at the same cross-shore transect for the plunging-breaker case

Figures 75 and 76 show results for the mean longshore current. Results suggest repeatability was good for the spilling-breaker case, considering the fact that small irregularities in the beach exist for each case (the beach does not have exactly straight and parallel contours). Also, small changes in topography might occur between runs as a result of the evolution toward equilibrium or due to beach perturbations that are created during the remolding process, which only can be performed to a certain level of accuracy and resolution. Results for the plunging-breaker case are not as good. This is attributed to the nonuniformities that developed in the region of the break-point bar. The beach was remolded after case $\mathrm{T} 3 \mathrm{~g}$ to a condition in which the bottom contours were straight and parallel in the bar region. Upon exposure to waves and currents, perturbations began to develop in the bar region and changed with time, i.e., from case to case.

Figure 77 illustrates the repeatability in sediment concentration measurements for the spilling-breaker case. The top panel shows measurements made in the incipient breaker zone, and the lower panel shows measurements from the midsurf zone. Concentrations are mean values, averaged over a 10-min sampling interval. Repeatability is reasonable and is, in general, better for higher concentrations that are measured closer to the bottom than for low concentration values measured higher in the water column. At elevations within $5 \mathrm{~cm}$ of the bed, where most of the longshore sediment flux occurs, mean concentrations are repeatable to within about $+/-25$ percent. 


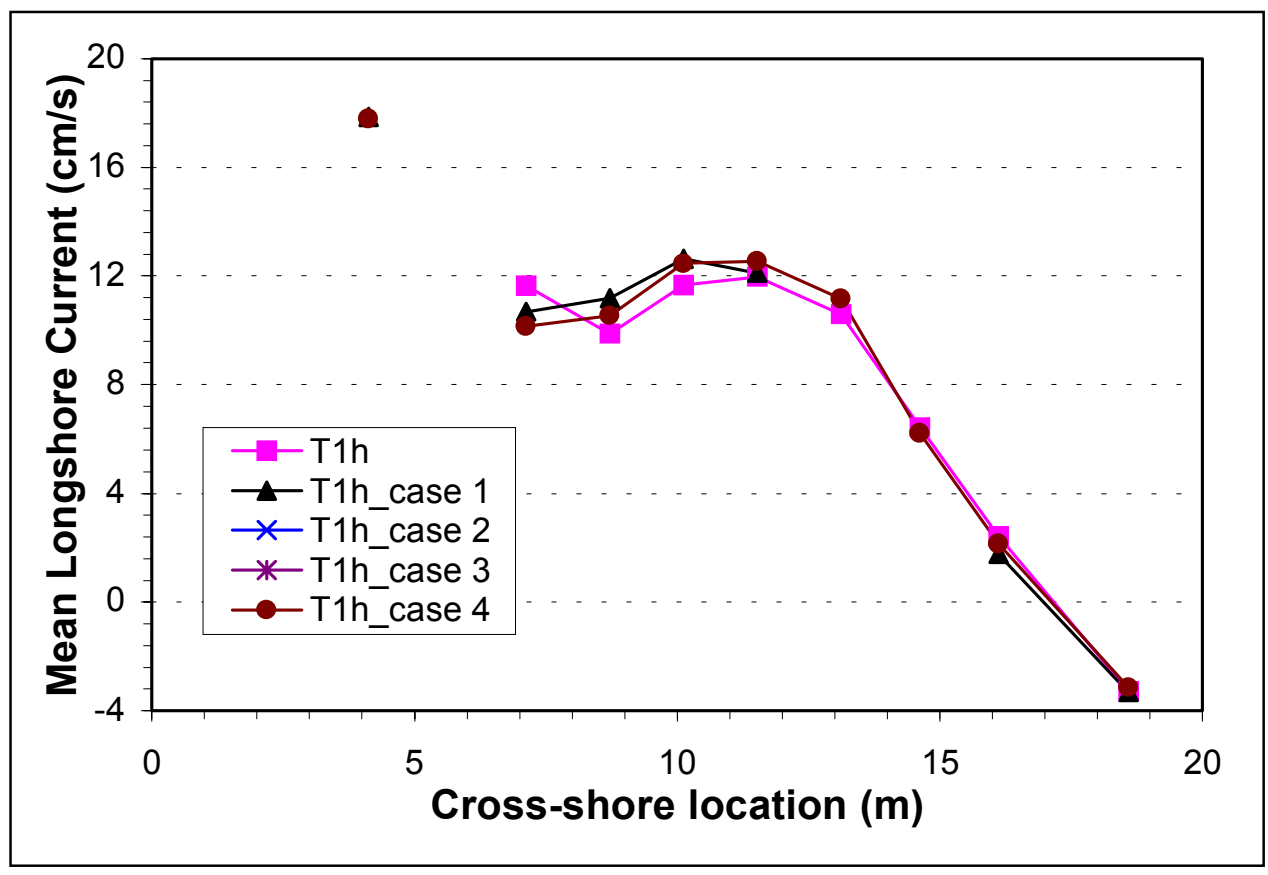

Figure 75. Measurements of mean longshore current for successive wave runs at the same cross-shore transect for the spilling-breaker case

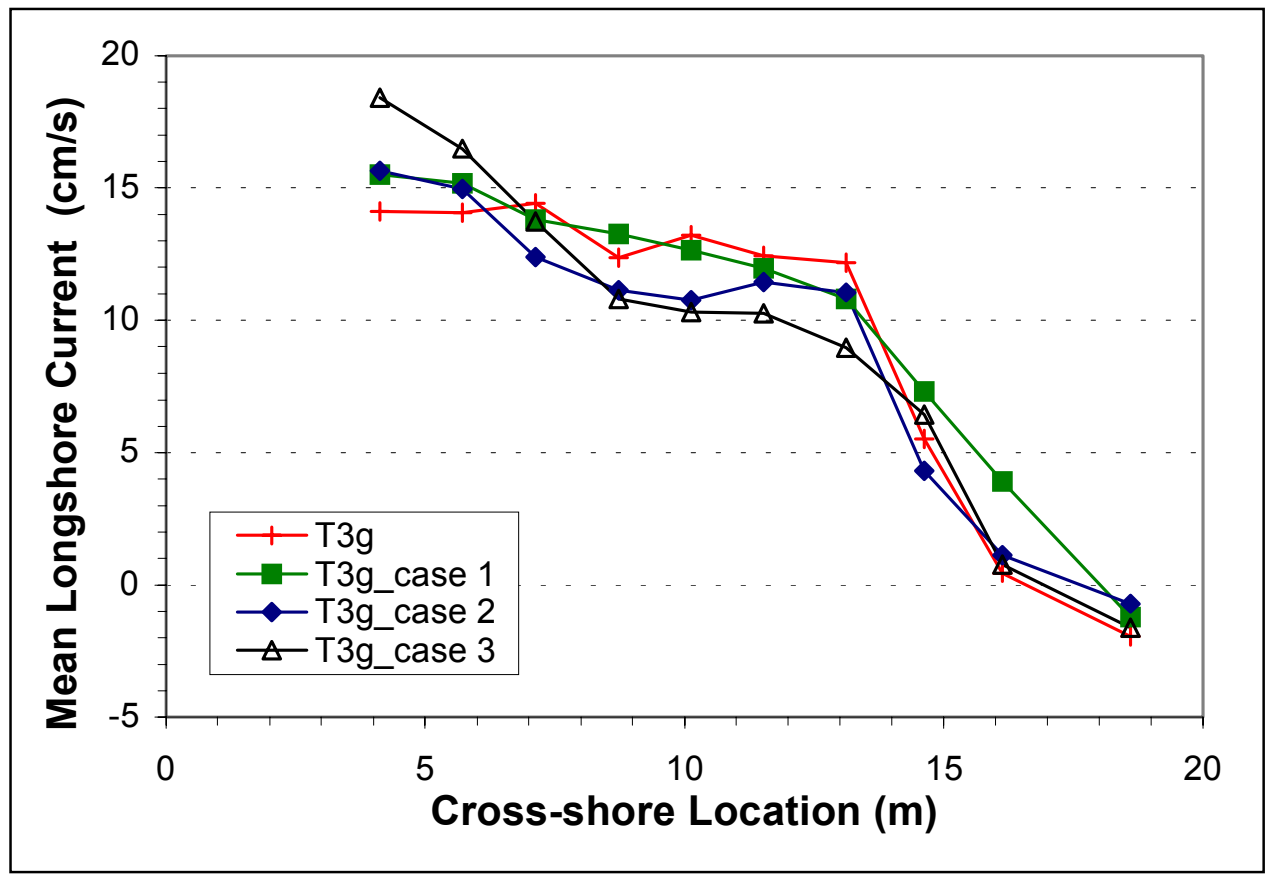

Figure 76. Measurements of mean longshore current for successive wave runs at the same cross-shore transect for the plunging-breaker case 

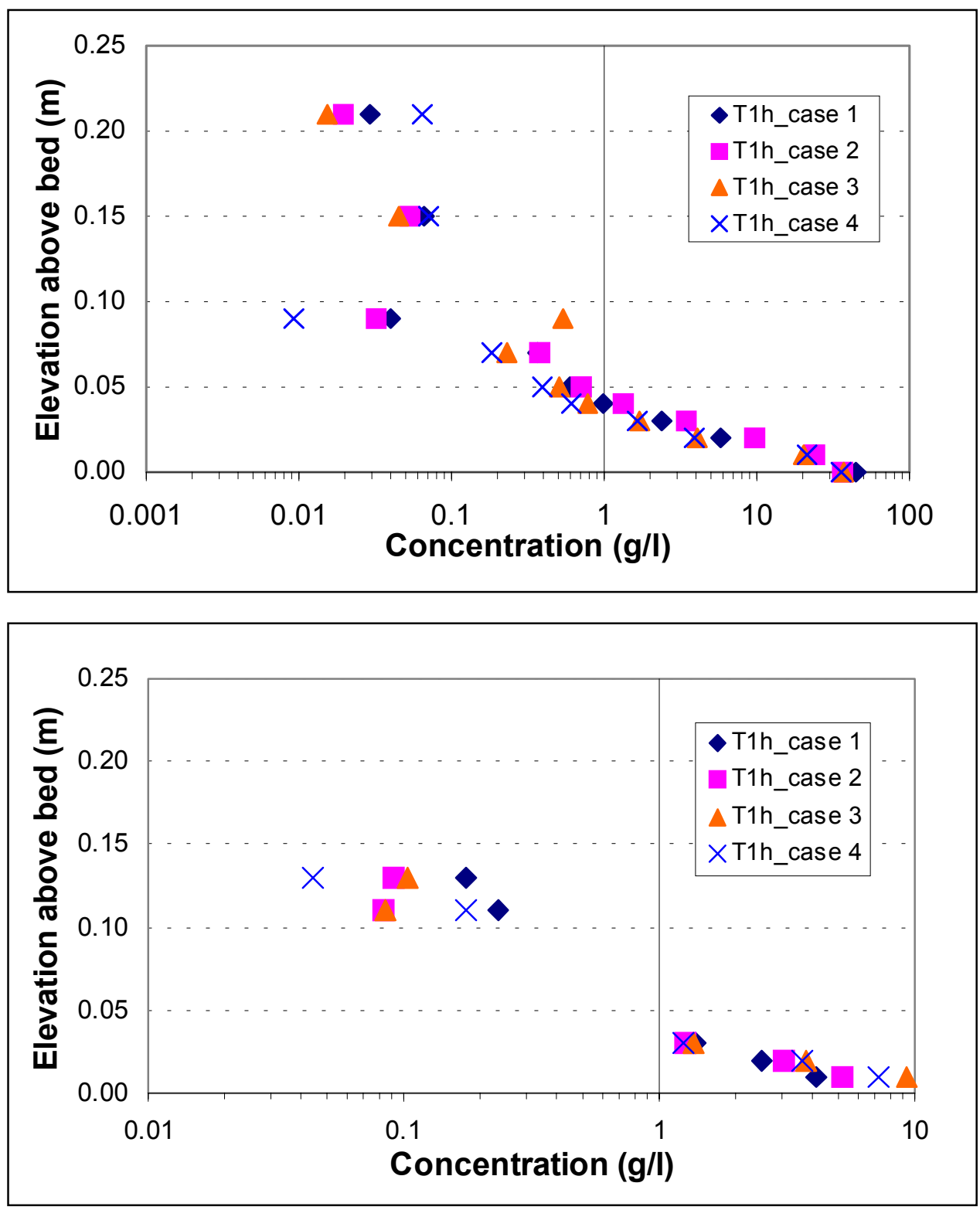

Figure 77. Measurements of sediment concentration for successive wave runs at the same cross-shore transect for the spilling-breaker case (top panel shows measurements from incipient breaker zone; bottom panel shows measurements from midsurf zone)

Figure 78 shows concentration measurements for the plunging-breaker case that were made at a position in the incipient breaker zone. In general, a greater degree of variability from run to run is evident for this wave condition. This also is attributed to differences in beach morphology that existed in the bar region for each of the cases. Repeatability in LST rates, based on trap measurements, is discussed later in this chapter. 

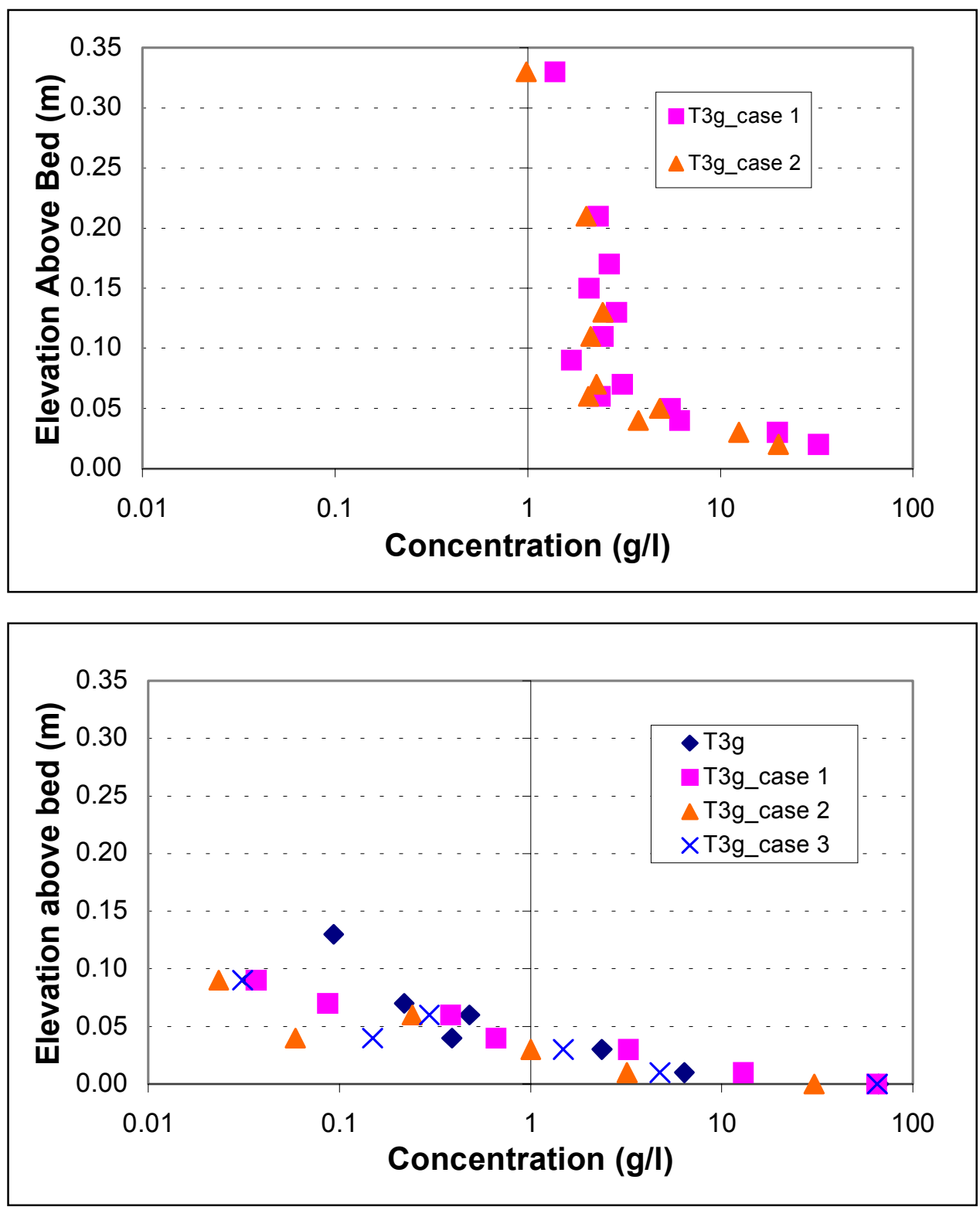

Figure 78. Measurements of sediment concentration for successive wave runs at the same cross-shore transect for the plunging-breaker case (top panel shows measurements from incipient breaker zone; bottom panel shows measurements from midsurf zone)

\section{Observed Beach Response}

\section{Spilling-breaker case}

Chapter 5 discussed procedures for molding the sand to produce a beach that has straight and parallel bathymetric contours. Figure 79 illustrates the condition of the beach after exposure to $6 \mathrm{hr}$ of wave and current forcing for the 
spilling-breaker wave case. This wave case was expected to produce a slightly erosive condition, characterized by loss of sand from the beach face and offshore movement of that sand, which did occur. At the beginning of this 6-hr wave run, the beach had already been subjected to numerous hours of wave action, had essentially reached its equilibrium profile shape, and had been remolded to the equilibrium profile shape with straight and parallel contours. The upstream boundary is located on the right hand side of the figure; and the sediment traps are located along the left side of the figure. Sand is transported alongshore, from right to left. Beach contour elevations are reported in tenths of meters, relative to the still-water line, which has an elevation of $0.0 \mathrm{~m}$.

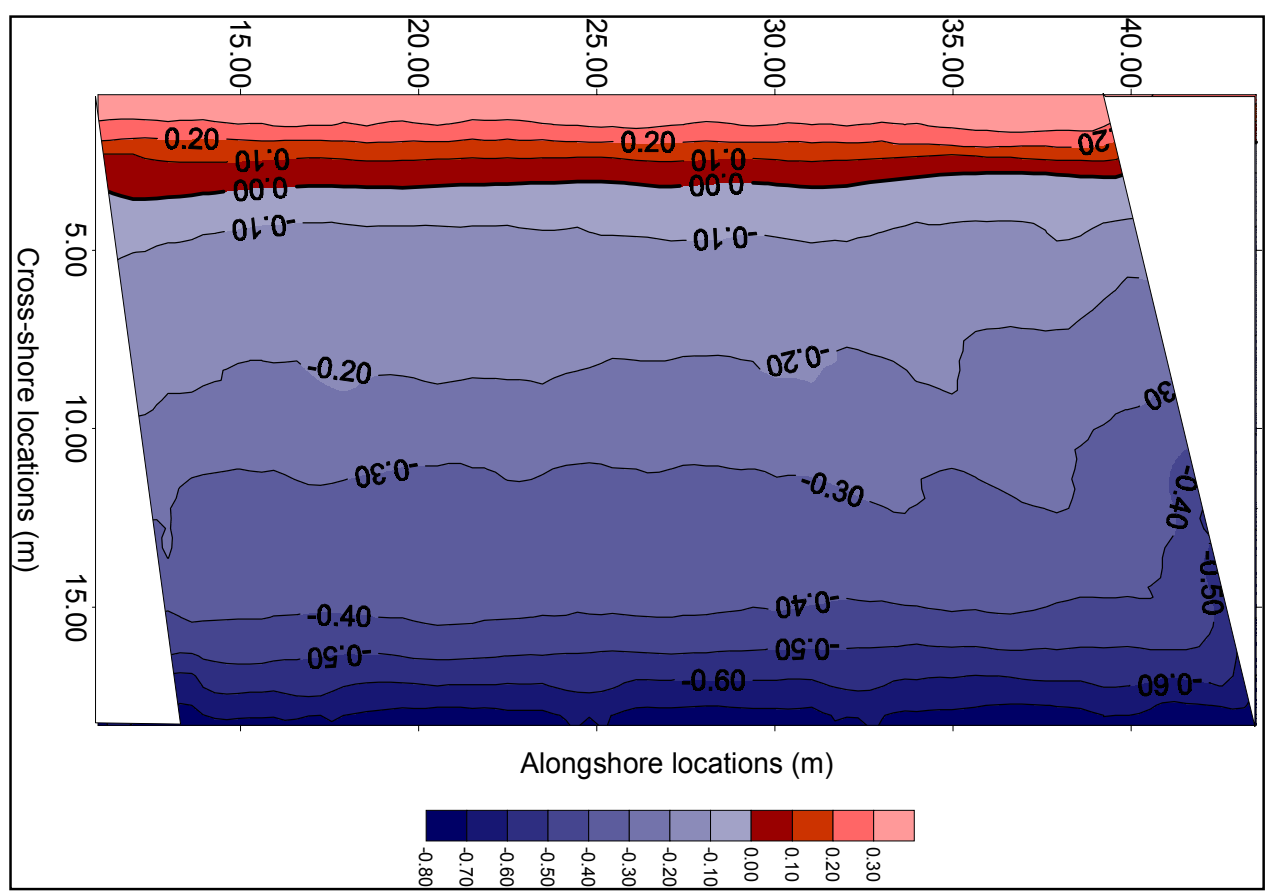

Figure 79. Beach morphology after $6 \mathrm{hr}$ of wave activity for the spilling-breaker case (elevations are in meters)

The effect of not introducing sand to the updrift boundary is clearly evident in Figure 79. Along the offshore portion of the updrift boundary, where the magnitude of longshore transport was rather low, the perturbation is very abrupt and is only evident within a distance of 1 to $2 \mathrm{~m}$ from the boundary. However, the perturbation extends a distance of 5 to $8 \mathrm{~m}$ from the boundary in the middle and inner regions of the surf zone, where the LST rates were higher. Where the deficit-induced perturbation transitions into the region of beach where straight and parallel contours are maintained, several undulations in beach morphology are evident. The undulations developed during every wave run following remolding of the beach. The reason for their occurrence is not definitely known. However, the difference in orientation between the updrift wave-guide (15 deg) and the angle of wave incidence at the wave generators $(10 \mathrm{deg})$ is suspected to be a contributing factor. The difference in angle was imposed in an attempt to compensate for wave energy that is lost through the updrift wave guide, thereby minimizing the alongshore extent of the current recirculation area that formed near the shoreline due to this energy loss. 
Along the shoreline, between alongshore coordinates of 33 and $39 \mathrm{~m}$, the foreshore beach slope is much narrower than along the rest of the shoreline (evident by more closely-spaced contours). A scarp formed along the seaward face of the beach berm in this region. No scarp was created along the rest of the shoreline. This anomalous erosion zone is also caused by the lack of a sediment supply at the updrift end of the beach. A peak in distribution of LST rate occurred in the swash zone.

Bathymetric contours remained reasonably straight and parallel between the alongshore coordinates of 15 and $30 \mathrm{~m}$. This is the same region that had the highest degree of alongshore uniformity in terms of longshore currents for the concrete beach tests. These two observations are probably related.

Along the downdrift boundary, slight changes in contour orientation are evident within several meters of the boundary. This is evidence that there is some anomalous erosion and/or accretion along this lateral boundary. The traps are not 100 percent efficient. These anomalies must be accounted for in estimates of LST rates that are derived from the trap weight measurements. The process for doing so is discussed in more detail later in this chapter.

In the offshore region, where the magnitude of the LST rate was low, the contours remained straight and parallel up to a point about $1 \mathrm{~m}$ away from the downdrift boundary. There, localized erosive perturbations are evident.

In the midsurf zone accretion fillets are evident, and the alongshore distance from the boundary that is affected increases with increasing proximity to the shoreline. The trap measurements indicated that the local LST rate increased with increasing proximity to the shoreline. This suggests that the magnitude of the accretive perturbation increases with increasing longshore sand transport rate. The same observation was noticed at the updrift boundary.

At the shoreline, an accretionary fillet is evident, but superimposed on it is a slight localized erosive area directly adjacent to the downdrift boundary. This is attributed to interactions of wave uprush with the bricks that comprise the adjustable downdrift beach template. The brick template holds the sand beach in place, preventing it from sloughing off into the sediment traps. In the foreshore zone, slight erosional perturbations are also evident. These seem to form for the same reason, interaction of wave uprush with the lateral boundary, which is discussed in more detail later in this chapter.

Figure 80 shows the condition of the same beach after exposure to nearly four additional hours of essentially the same wave and current conditions. The beach morphology seen in Figures 79 and 80 is quite similar. The erosion perturbations at the updrift end of the beach grew in severity as would be expected, since no sand was supplied; but the alongshore extent of the perturbation did not change appreciably. At the downdrift end of the beach, the regions of localized accretion and erosion did not change much during the additional $4 \mathrm{hr}$. The perturbations seemed to reach a near-equilibrium condition, presumably with sand being transported into the traps more efficiently once this condition is reached. 


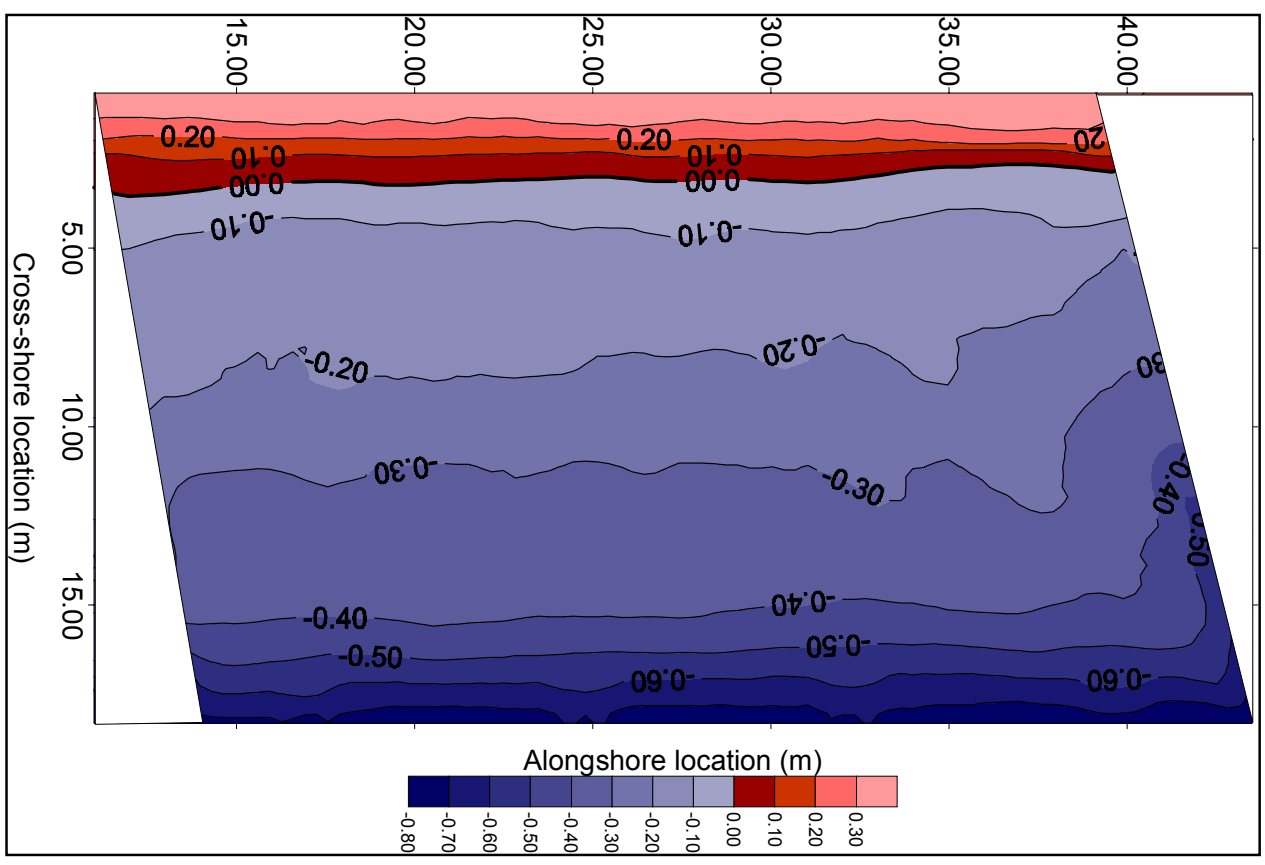

Figure 80 . Beach morphology after nearly $10 \mathrm{hr}$ of wave activity for the spillingbreaker case (elevations are in meters)

Figure 81 shows the cumulative change in beach elevation for this same case during the $10 \mathrm{hr}$ of exposure to the same spilling-type breaking waves. Elevation changes are given in meters. The loss of sand from the updrift end of the beach is quite evident; beach elevation decreased by $0.1 \mathrm{~m}$ or more in the high-loss areas. Figure 81 clearly shows the alongshore extent of the perturbations both in the offshore region and along the beach, and how the extent varies with crossshore position. The figure shows that the inshore portion of the profile (yellowshaded region) continued to lose between 0 and $2 \mathrm{~cm}$ of elevation, while the offshore bar gained sand at about the same rate (green-shaded region). This appears to be a consequence of the continued approach toward an equilibrium beach condition, albeit at a much slower rate that that observed in the early stages of profile evolution. The maximum bottom elevation increase, associated with accretion along the downdrift boundary, was about $0.04 \mathrm{~m}$. 


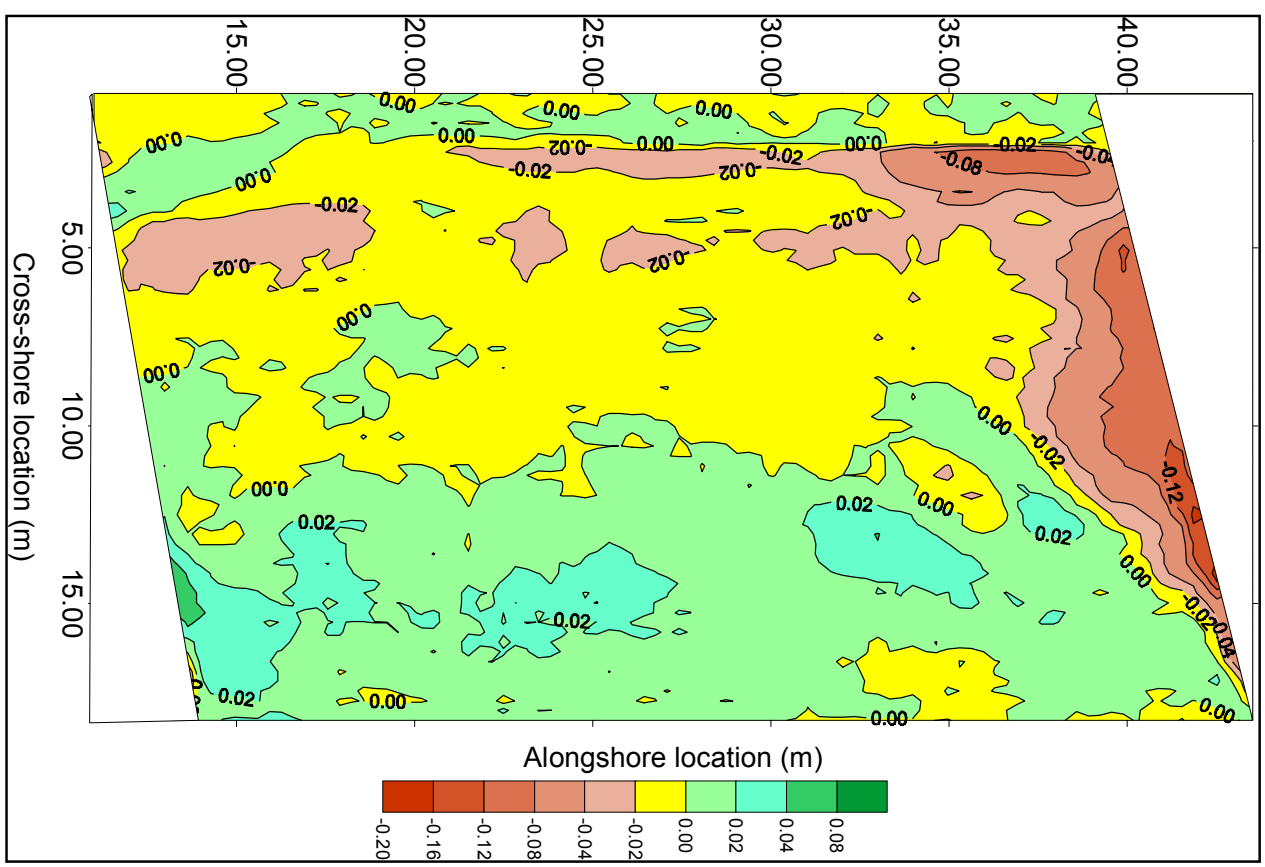

Figure 81 . Change in morphology after nearly $10 \mathrm{hr}$ of wave activity for the spilling-breaker case (elevation changes are in meters)

\section{Plunging-breaker case}

Figure 82 illustrates the condition of the beach after approximately $3.3 \mathrm{hr}$ of wave activity, in response to the plunging-type breaking waves and associated longshore currents. This figure shows the bathymetric change that occurred. The wave conditions were expected to produce a pronounced breakpoint bar, which they did. These plunging wave conditions produced about 2.6 to 2.7 times more longshore sand transport, compared to the spilling-breaker wave case; and the cross-shore distribution of longshore transport was quite different. The plungingbreaker case produced two peaks in the LST distribution, one at the breaker zone and one in the foreshore. The spilling-breaker case produced a distribution in which the magnitude of LST monotonically increased with increasing proximity to the shoreline, reaching its peak value in the foreshore.

In many ways, beach changes observed during the plunging-breaker case were similar to those observed for the spilling-breaker case; but there were some differences. In the most offshore portion of the updrift end of the beach, a slight erosion perturbation is evident in Figure 82, and it is confined to a relatively short distance away from the edge of the beach. This same observation was noted for the spilling-wave case. 


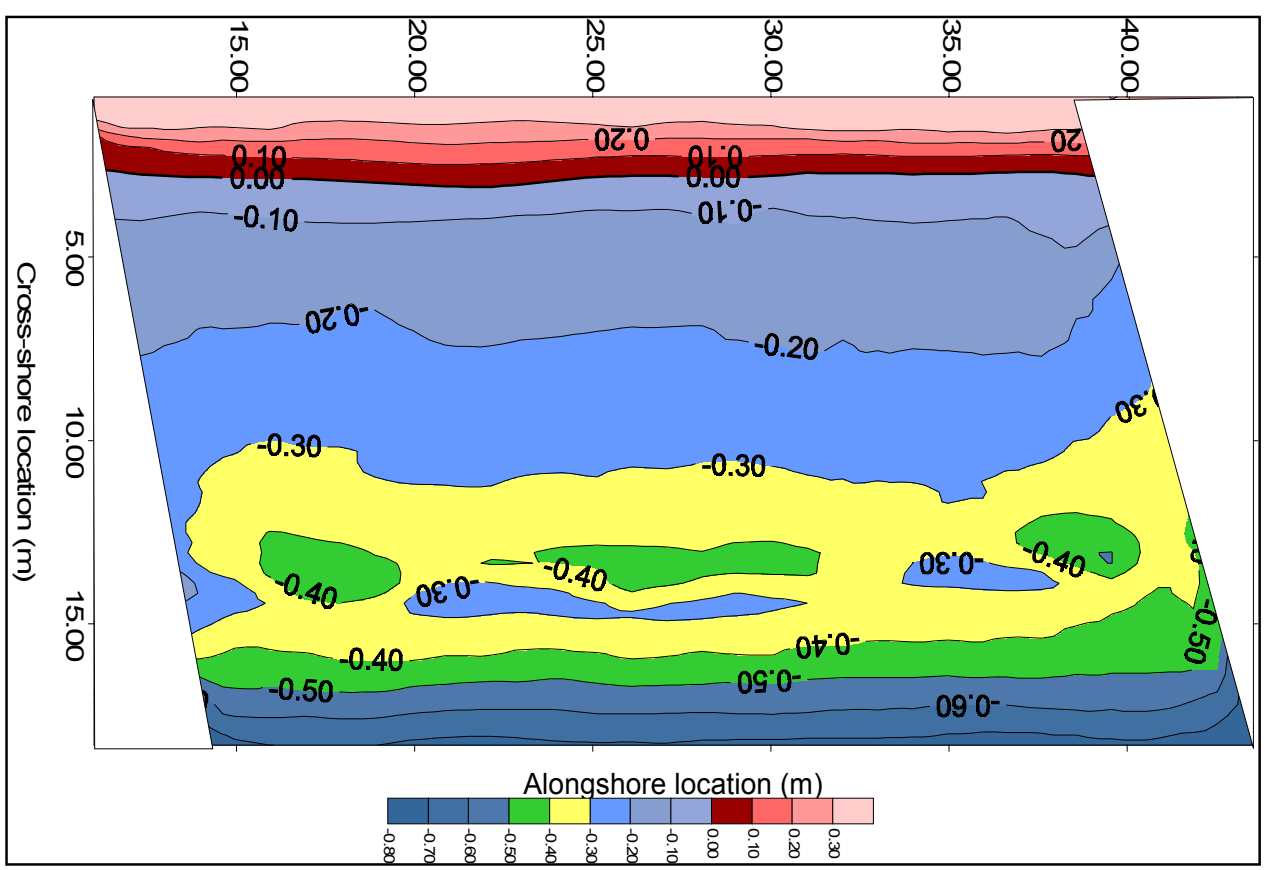

Figure 82. Beach morphology after nearly $10 \mathrm{hr}$ of wave activity for the plungingbreaker case (elevations are in meters)

As was found for the spilling wave case, the erosion perturbation is evident along nearly the entire updrift boundary. In the vicinity of the bar, the perturbation is greatest in magnitude and in terms of extent from the boundary. The perturbation influences the orientation and crest elevation of the bar in this region. A peak in the longshore transport distribution existed in the vicinity of the bar for the plunging-breaker case. As was found for the spilling-wave case, the magnitude and alongshore extent of the erosion perturbation was greatest in areas where the LST rate was highest. For the plunging wave case there is erosion of the shoreline along the updrift boundary, and the upper beach slope contours are narrowed, as was seen for the spilling wave case. Foreshore and shoreline erosion perturbations appear to extend further downdrift than was observed in the spilling-breaker case, approximately to the 30-m alongshore coordinate, consistent with the fact that high transport rates were evident in the swash zone for the plunging-breaker wave case.

Unlike the spilling-breaker case, no well-defined undulatory morphologic features existed at the point where the updrift erosion perturbations transition to the section of beach having relatively straight and parallel contours. Bottom contours in the vicinity of the bar show much more irregularity than offshore contours in the same depths measured in the spilling wave case. The bar evolved after remolding, and this evolution is believed to explain the lower degree of repeatability in process measurements that were made for the plunging-breaker case. With the exception of the bar region, beach contours are reasonably straight and parallel between the alongshore coordinates of 15 and $30 \mathrm{~m}$.

Along the downdrift boundary of the beach, a slight erosional perturbation exists along the offshore end of the beach, as was seen for the spilling-breaker 
case. Along much of the rest of the surf zone, an accretional fillet is evident, with the fillet at the bar location being the most pronounced. This location corresponds to the peak in the distribution of the LST rate. The presence of a pervasive accretion fillet also was observed for the spilling wave case. Near the shoreline, an erosion perturbation is evident, not an accretion fillet. This is different from the spilling-wave case. Its occurrence is attributed to the more active swash zone associated with the longer-period waves and the stronger interaction of wave uprush associated with the bricks used to construct the downdrift profile template.

\section{Alongshore Uniformity of Processes}

\section{Beach profile shape}

For both the spilling- and plunging-breaker cases, reasonably straight and parallel contours were maintained in the region of beach between alongshore coordinates of 15 and $30 \mathrm{~m}$. This is the same region that had the greatest degree of alongshore uniformity, in terms of waves and mean currents, for the fixed-bed concrete-beach experiments. A slight bar feature formed in the vicinity of the incipient breaker line, in the region between cross-shore coordinates of 13 and 16 $\mathrm{m}$ in for the spilling-breaker case. A pronounced break-point bar formed in nearly the same location for the plunging-breaker case. Both cases had a similar incident wave height, but peak periods differed substantially. Throughout most of the mid- and innersurf zones, beach profile shapes were quite similar. The foreshore beach slope was slightly greater for the plunging wave case.

Figures 83 and 84 illustrate the alongshore variability in beach profile shape for the spilling and plunging-wave cases, respectively. The beach profiles depicted in these figures were measured after the beach had reached an equilibrium condition (or very nearly so) and the proper mean longhore currents were being pumped. The upper panel in each figure shows beach profiles, at $1-\mathrm{m}$ intervals, between the alongshore coordinates of 15 and $30 \mathrm{~m}$. Note that the still water level is at an elevation of $0.9 \mathrm{~m}$. The lower panel shows the mean profile shape computed from all profiles in this section of the beach, along with envelopes representing $+/$ - one standard deviation of elevation difference between the various profiles and the mean profile. The degree of alongshore uniformity is high, particularly for the spilling-breaker case. Uniformity for the plunging wave case is also good, but there is more alongshore variability in the vicinity of the bar. This is also evident in Figure 82, which shows the beach contours for this case.

\section{Waves and currents}

Figures 85 and 86 illustrate the degree of alongshore uniformity in significant wave height, and Figures 87 and 88 illustrate uniformity in mean longshore current, for the spilling and plunging wave cases, respectively. The proper longshore current was being pumped in both cases. Wave and current parameters displayed in the figures were computed for a 10-min sampling record. The 

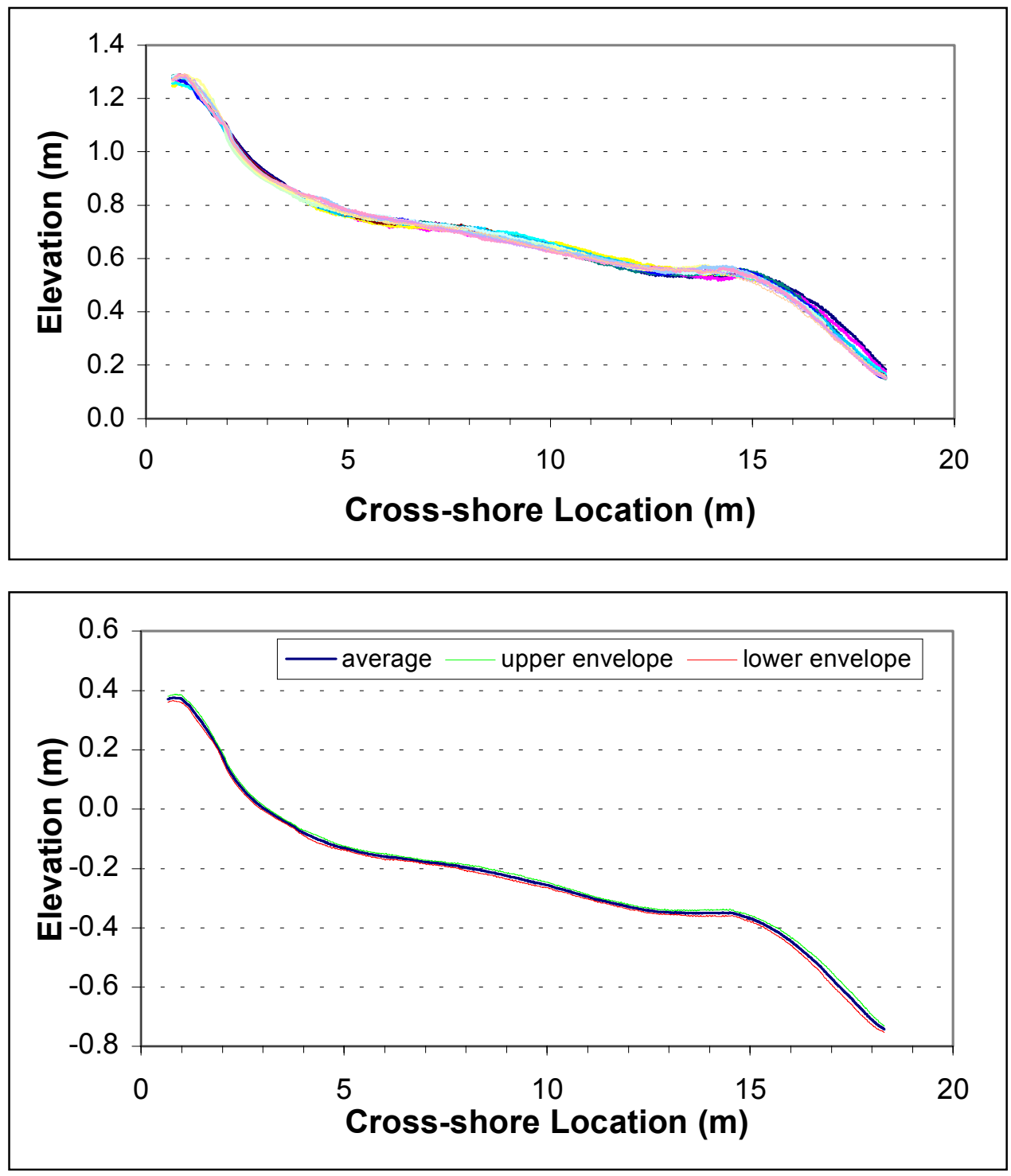

Figure 83. Alongshore variation in beach profile shape for the spilling-breaker case (in the upper panel, still-water level is at an elevation of $0.9 \mathrm{~m}$ ) 

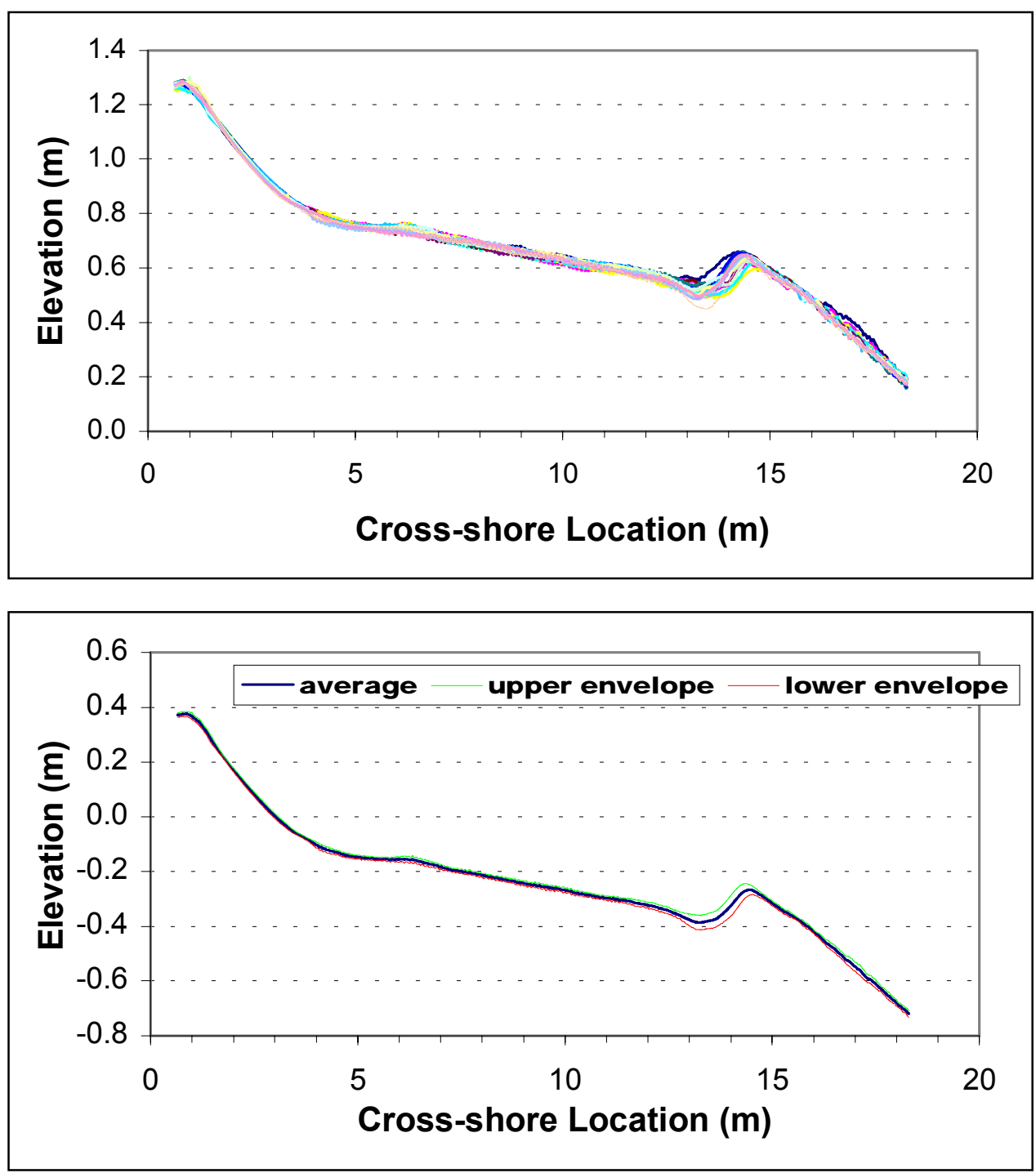

Figure 84. Alongshore variation in beach profile shape for the plunging-breaker case (in upper panel, still-water level is at an elevation of $0.9 \mathrm{~m}$ )

"Y..." locations listed in the legend indicate the alongshore coordinate, in meters. Most process measurements (waves, currents, and sediment concentration) were made in the region where the beach had the highest degree of alongshore uniformity, between alongshore coordinates of 15 and $30 \mathrm{~m}$. In this region, the degree of alongshore uniformity in terms of these hydrodynamic parameters is good, and comparable to that which was achieved for the fixed-bed, concrete-beach experiments.

Significant wave heights generally varied within the range of plus or minus 10 percent of the mean values computed using data from all transects.

Variability is greatest in the incipient breaking zone. Significant wave height along one of the transects was noticeably different for the plunging-breaker case. Otherwise, a similar degree of longshore variability was observed in both spilling and plunging wave cases. Variability was higher for the plunging-breaker case in 
the offshore portion of the beach profile, which is consistent with the higher degree of bathymetric variability in the bar region. Variability in the innersurf zone was much less.

Mean longshore currents generally varied within plus or minus 20 percent of the mean computed for all transects, except in the offshore tail of the distribution and very near the shoreline where variability was much greater. Overall, the degree of alongshore uniformity in hydrodynamic parameters was good, but slightly less than that achieved for the concrete-beach experiments. This was expected as a result of the mobility of the bed, irregularities in beach morphology, and recognized imperfections in lateral boundary conditions.

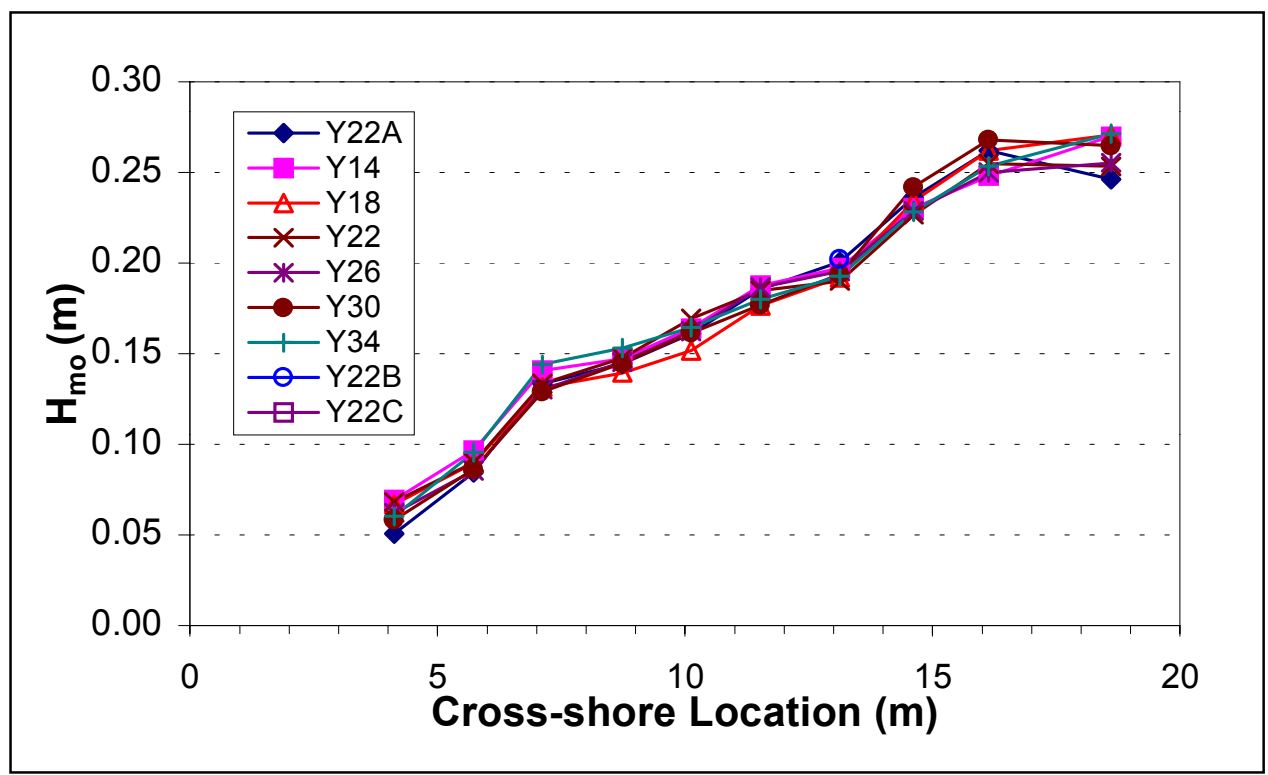

Figure 85. Alongshore variation in significant wave height for the spilling-breaker case 


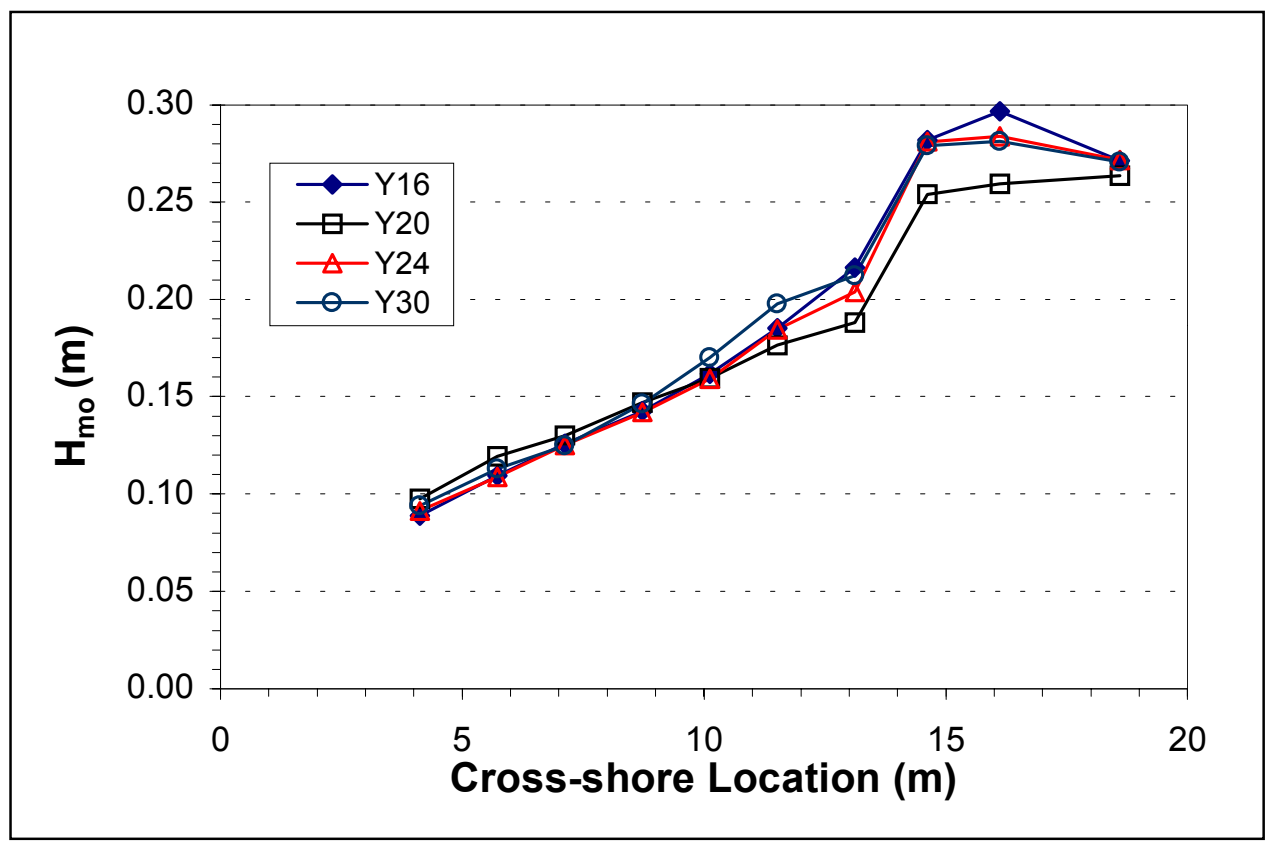

Figure 86. Alongshore variation in significant wave height for the plungingbreaker case

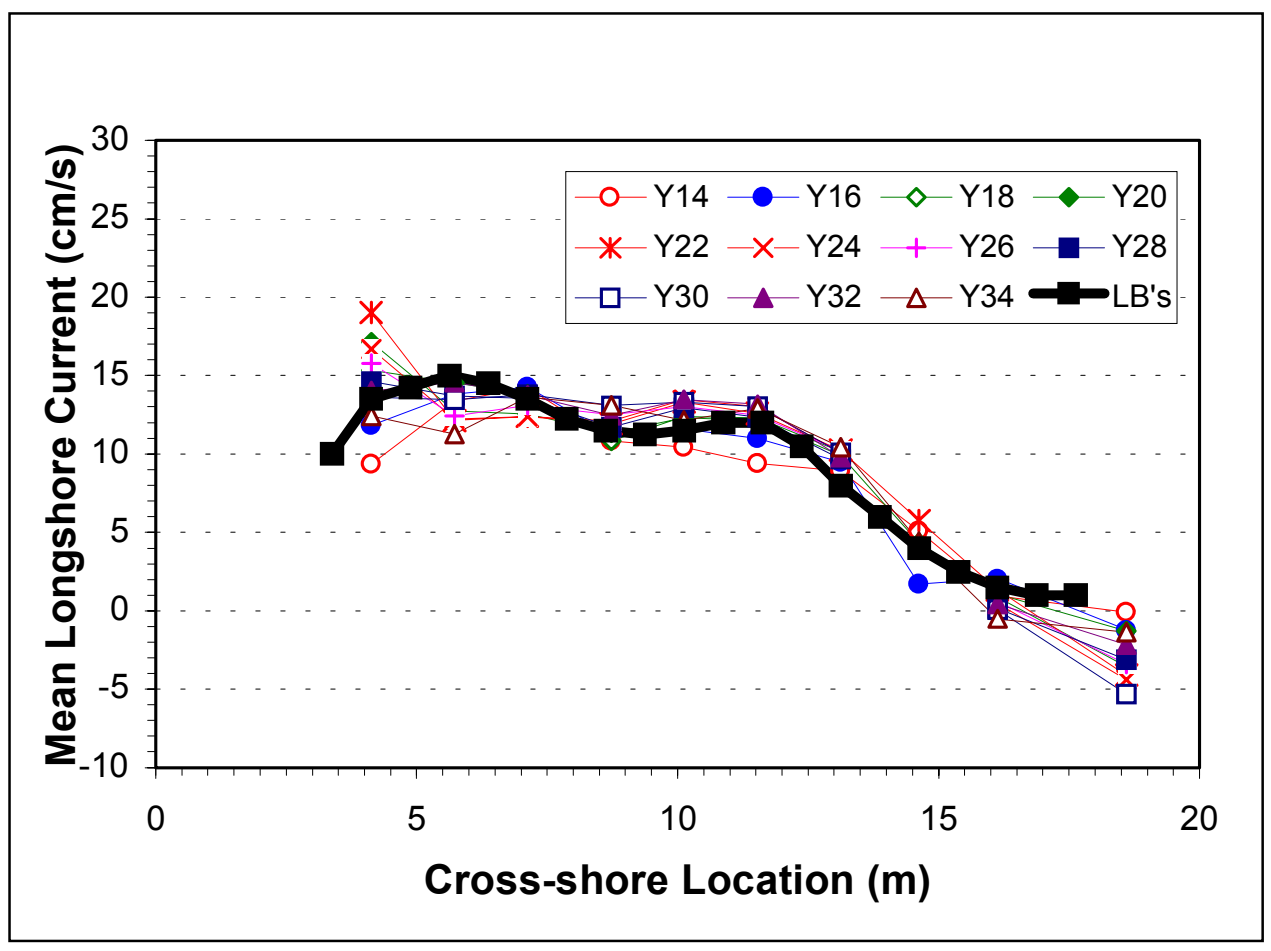

Figure 87. Alongshore variation in mean longshore current for the spillingbreaker case 


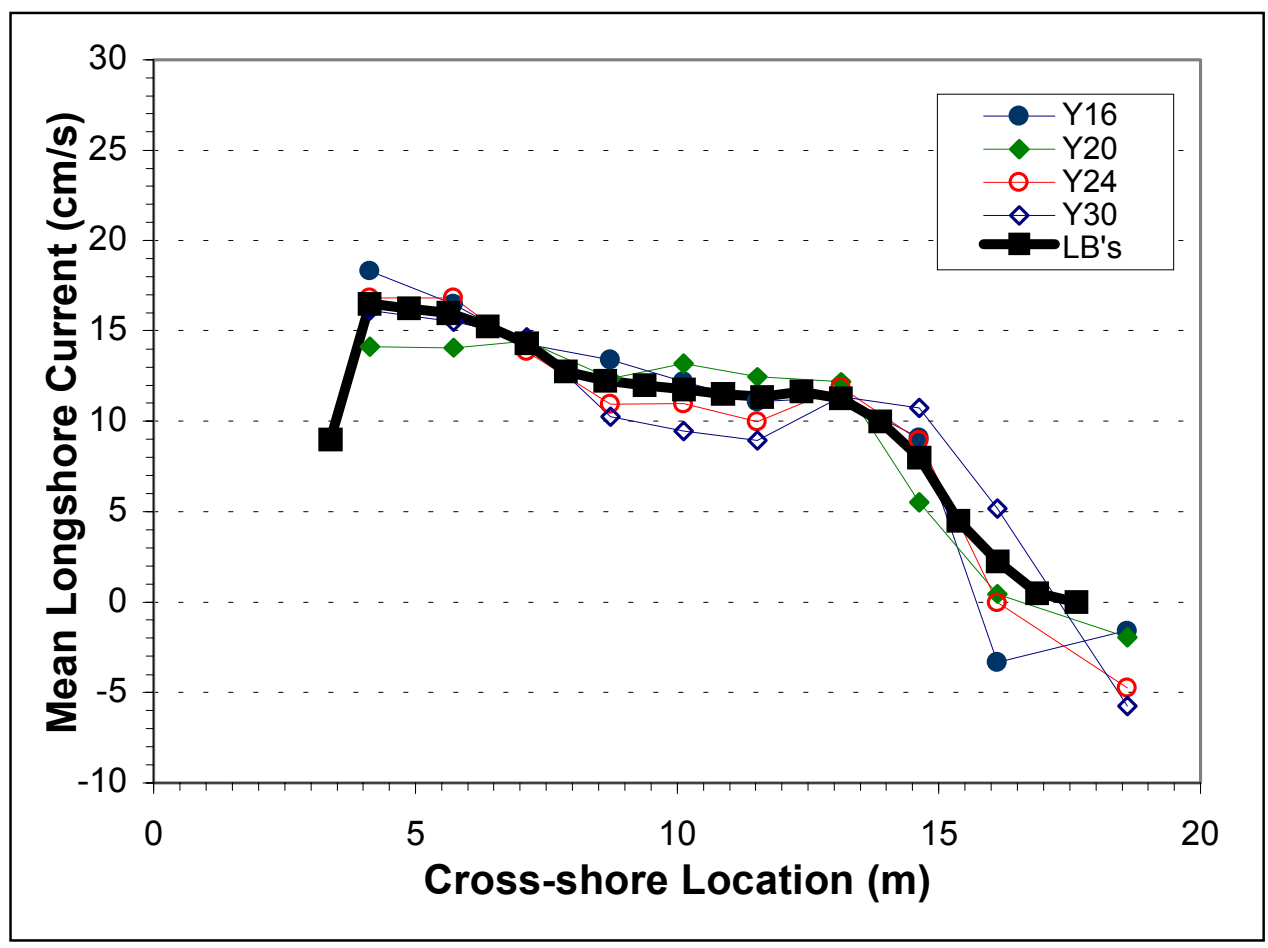

Figure 88. Alongshore variation in mean longshore current for the plungingbreaker case

\section{Sediment concentration}

Figures 89 and 90 illustrate the degree of alongshore uniformity in measured sand concentration for the spilling- and plunging-breaker cases, respectively. Both the top and bottom panels of each figure show the temporally averaged sediment concentration (a mean computed for a 10-min record) as a function of elevation within the water column at a number of transects along the beach. Measurements were repeated three times at each transect for the spilling breaker case, and those data also are shown in Figure 89. At each alongshore position, concentrations are given for two locations, one in the incipient breaker zone (top panel in each figure), and one in the midsurf zone (bottom panel in each figure). Note that the vertical axis is plotted using a logarithmic scale.

For the spilling-breaker case, concentrations varied by 20 to 40 percent of the mean value in the alongshore direction for measurements made in the zone of incipient wave breaking. Variability was higher in the midsurf zone, with variability ranging from 30 to 90 percent of the mean. A similar result was found for the plunging-breaker case. In the incipient breaker zone, variations were generally 10 to 30 percent of the mean, with variability approaching 50 percent for a few sensors. In the midsurf zone, variability generally ranged from 30 to 45 percent of the mean, with some sensors differing by as much as 120 percent (near the surface in this instance). Variability in measured mean concentration is much higher than variability in gross wave and current parameters. This suggests that concentration measurements from a number of transects should be averaged, 
to represent sediment concentrations throughout the region having the highest alongshore uniformity.
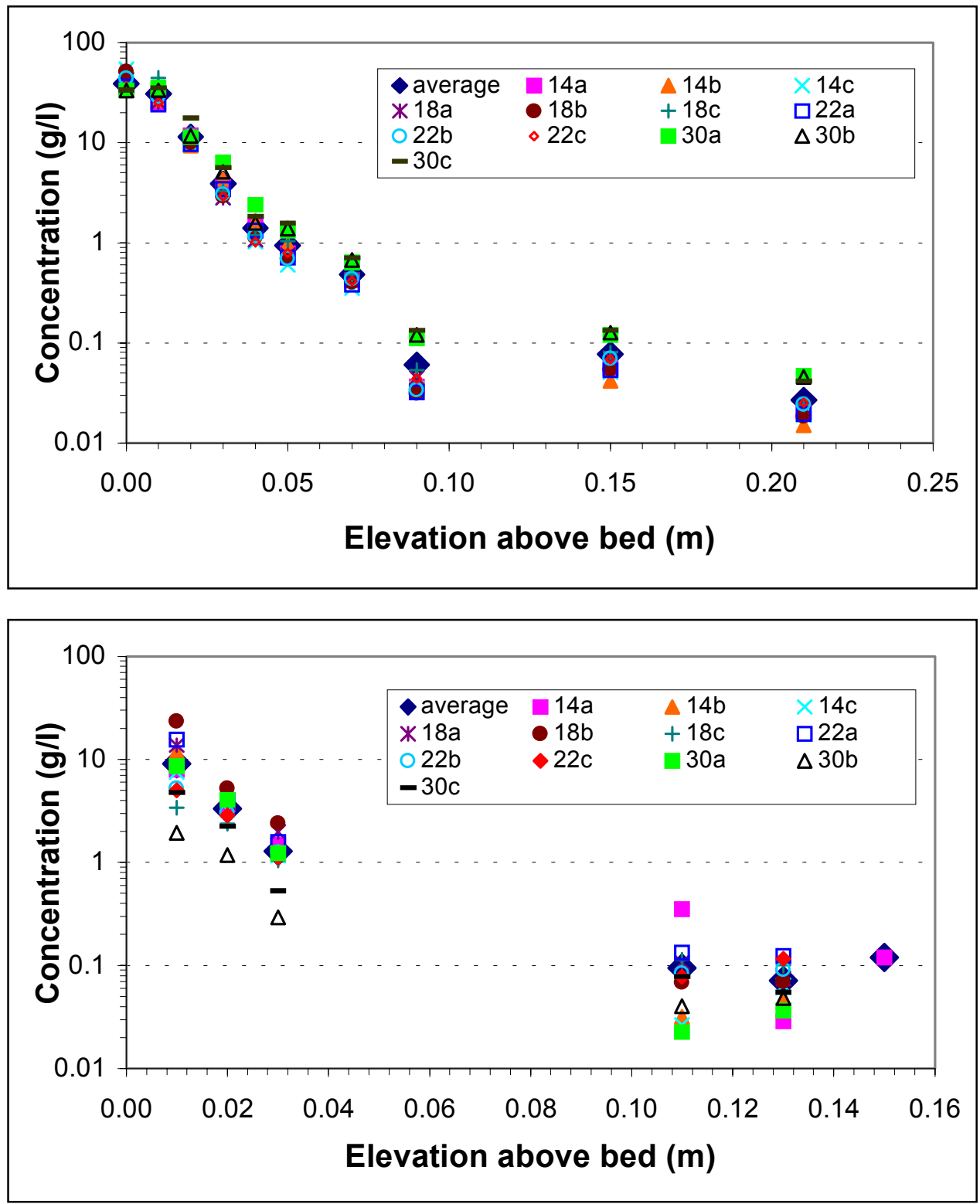

Figure 89. Alongshore variation in time-averaged mean sediment concentration for the spilling-breaker case (top panel shows results from the incipient breaker zone; bottom panel shows results from the midsurf zone) 

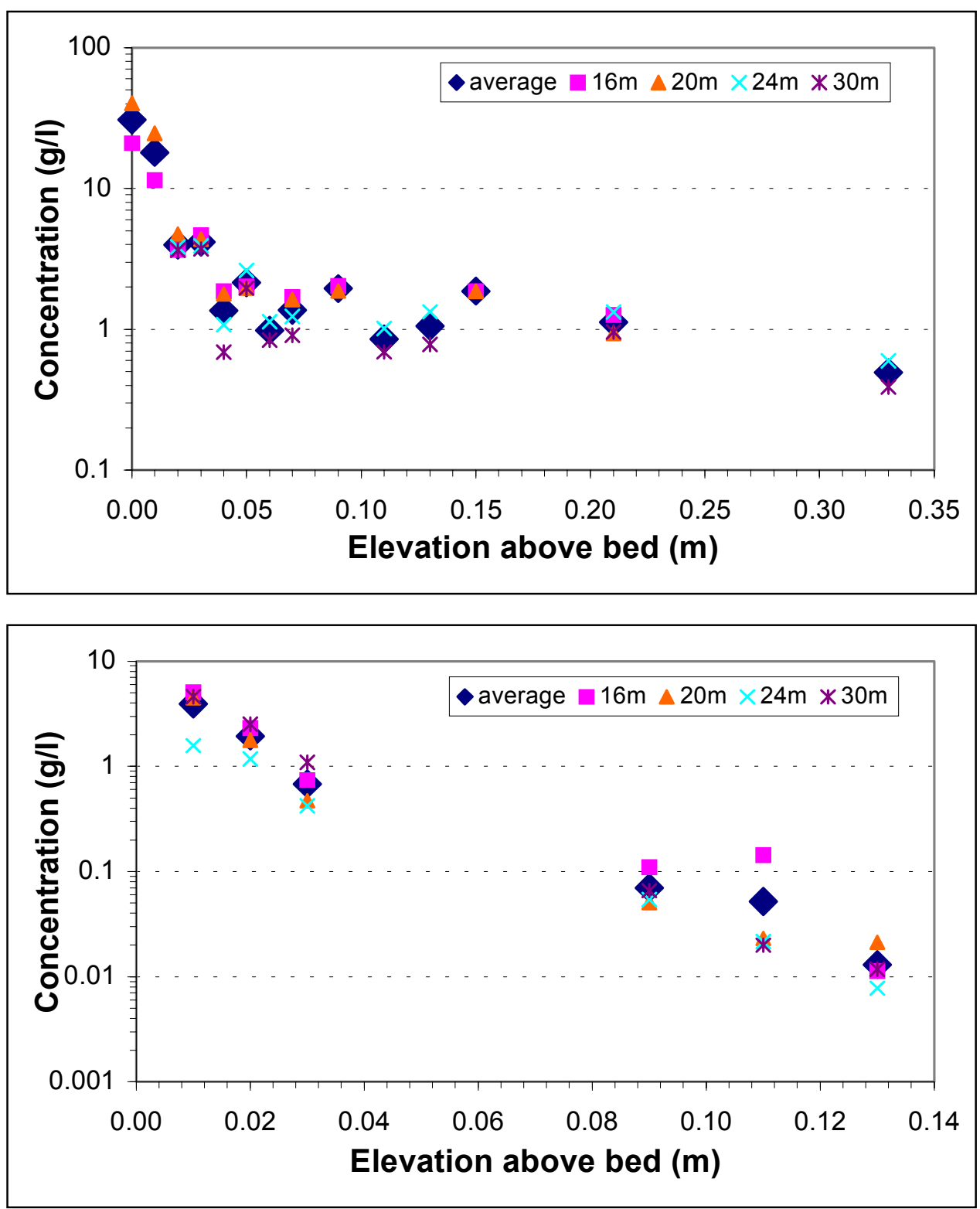

Figure 90. Alongshore variation in time-averaged mean sediment concentration for the plunging-breaker case (top panel shows results from the incipient breaker zone; bottom panel shows results from the midsurf zone)

\section{Performance of the Sand Traps}

Several issues related to trap effectiveness were raised and discussed in Chapter 8: The quantity of sand that remains suspended in the water column and is transported past the traps, accumulation of sand on the rubber neoprene seals around the periphery of each trap (both sides and the updrift edge), and corrections to the trapped quantities that must be made as a result of anomalous beach responses adjacent to the downdrift boundary. These sources of error must 
be considered in deriving estimates of the LST rate. The maximum error associated with sand that bypasses the trap is about 2.5 percent, but this magnitude of error occurs only in flow channels with the highest longshore transport rates (high longshore currents and high levels of sand entrainment). Measurements to quantify this error were made under some of the most energetic wave and current conditions that are to be generated in the facility. In most channels, especially those in the vicinity of the shoreline, the error is much less than this value. Therefore, sand bypassing should only have minimal influence on the estimates of LST. The last two issues are discussed in more detail here, because they are potentially the largest sources of error in the measurements of trap weight, and consequently, estimates of LST that are made based on the weight of sand that accumulates in the traps.

Sand accumulates on the neoprene rubber seals around the periphery of the sand traps. Figure 91 provides a representative example of the magnitude of these quantities, as a percentage of the total weight that accumulated in the traps, for the plunging-breaker case. Two quantities are shown: one represents sand that accumulated on the side seals, and one reflects sand that accumulated on the front, or updrift, seal. Generally, the quantity of sand that deposits on the updrift seal greatly exceeds that which accumulates on the side seals. However, for a few of the traps shown in the figure, the two quantities are comparable. The updrift seals do not have uniform surface areas. Generally, the total quantity that accumulates on all rubber seals is about 3 to 12 percent of the total that actually settles into the trap. As seen in the figure, the percentage of sand accumulating on the seals can approach 15 to 20 percent in individual traps. Results shown in Figure 91 are similar to those observed for the spilling breaker case (see Figure 52).

Earlier in this chapter, the anomalous 3-D beach evolution that occurred at the downdrift boundary was discussed. These anomalies must be accounted for, as corrections to the measured trap weights, to maximize the accuracy of computed LST rates. The extent and magnitude of the anomalies change with cross-shore position; therefore, the magnitude of the corrections varies with cross-shore position. In general, anomalies were restricted to the region of beach within 1 to $3 \mathrm{~m}$ of the downdrift boundary. The method used to correct trap weights is illustrated using Figure 92, and described below.

Figure 92 shows the total volume change on each of the beach profiles measured between the alongshore coordinates of 11 and $44 \mathrm{~m}$ (representing the entire length of beach). Profiles were measured at 0.5 -m intervals. A persistent pattern of volume loss is evident at the updrift end of the beach, between alongshore coordinates of 32 and $44 \mathrm{~m}$, which arises because no sand is introduced at the updrift boundary during the experiments. Between alongshore coordinates of 16 and $32 \mathrm{~m}$, small changes are evident but no persistent volume gains or losses are obvious. This is the region where the highest degree of longshore uniformity in process measurements is observed, and the region where the bathymetric contours remain nearly straight and parallel. At the downdrift end of the beach, between alongshore coordinates of 11 and $16 \mathrm{~m}$, larger volume changes are evident. Volume changes in this zone are assumed to be anomalous and caused by lateral boundary imperfections. These volume changes are used to develop corrections to the quantities of sand that accumulate in the traps. 


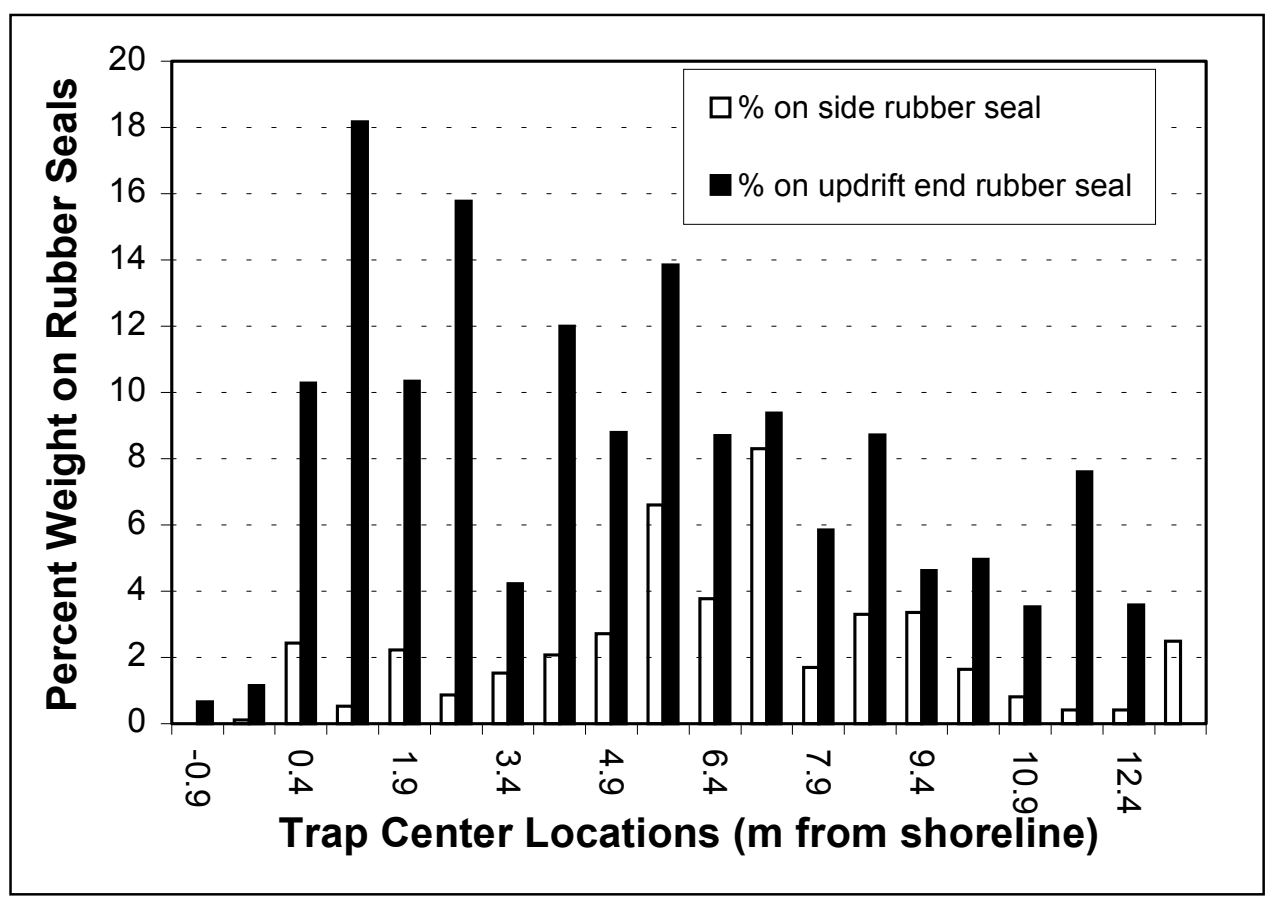

Figure 91. Weight of sand that accumulated on the rubber seals around the traps

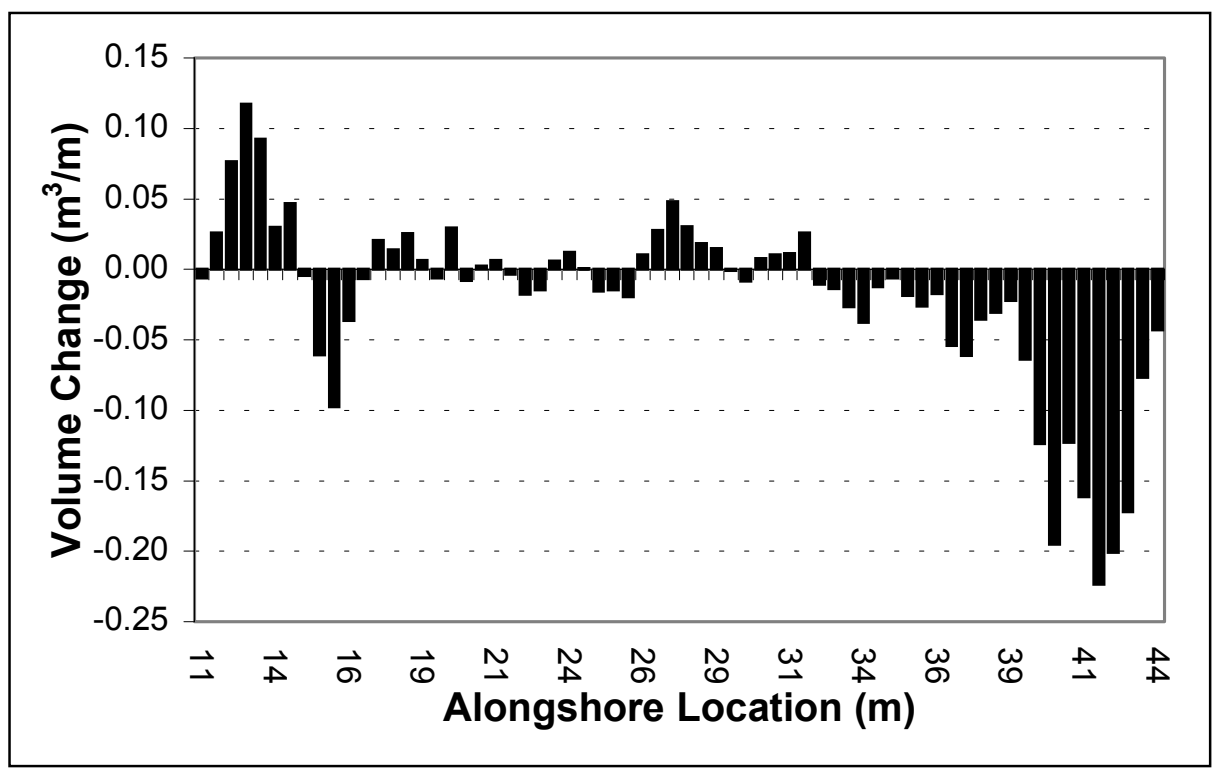

Figure 92. Volume change computed for each profile

Within each $0.75-\mathrm{m}$ cross-shore section of beach in this anomalous zone ( $0.75 \mathrm{~m}$ is the width of each flow channel), the measured trap volume for that channel is adjusted to reflect the anomalous volume change within that section of beach. Trap weights are converted to volume by considering the porosity of wet sand that accumulates in the traps (porosity of 0.40 is assumed). Generally, volume changes between the downdrift boundary and the alongshore coordinate 
of $15 \mathrm{~m}$ are considered. Where anomalous erosion occurs, the correction is subtracted from the volume that accumulates in the trap; where accretion occurs, the correction is added to the volume in the trap. All trapped quantities are corrected in this manner.

Figures 93 and 94 show the total rate of LST, with and without these corrections, for spilling- and plunging-breaker cases, respectively. Results for a number of wave runs, or test cases, are shown in each figure. In each figure, all tests involve the same wave forcing, and are shown in chronological order. Early runs, T1a through T1g, and T3a through T3i_case 1, involve iterations to determine the proper pump settings, and are not relevant to this discussion.

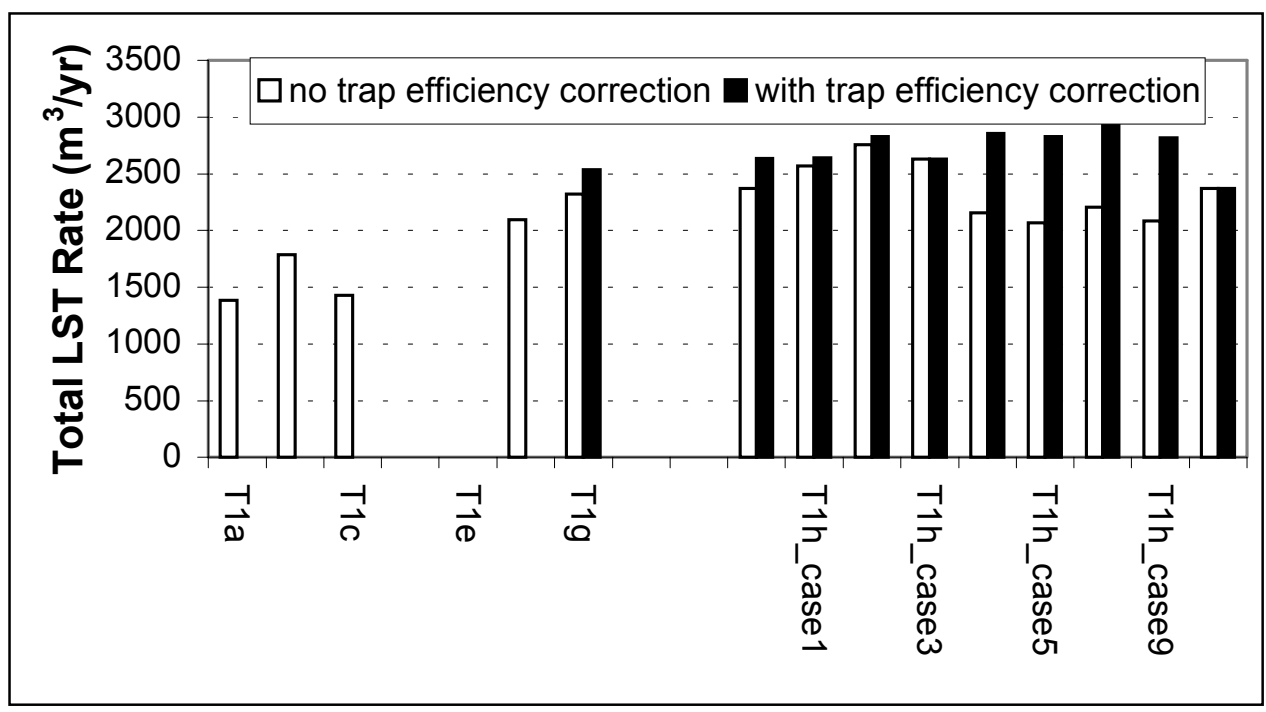

Figure 93. Total LST rates computed with and without corrections to account for anomalous volume change adjacent to the downdrift boundary for the spilling-breaker case

In Figure 93, run T1h is the first run in which the "proper" mean longshore currents were pumped in all flow channels for the spilling-breaker case.

Subsequent runs, T1h_case1 through $\mathrm{T} 1 \mathrm{~h} \_$case 4 were made for various purposes, but with the same mean longshore current distribution as run T1h. Cases T1h_case5 through T1h_case10, were made with nearly the same mean longshore currents as cases 1 through 4, but currents were modified slightly to correct for small changes in mean beach profile shape. Theoretically, the total corrected transport rates for cases T1h through T1h_case4 should be the same, and results for cases T1h_case 5 though T1h_case 10 should be very similar, perhaps with slight differences. It is important to note that the beach was rebuilt prior to cases T1h_case 4 and T1h_case8. 


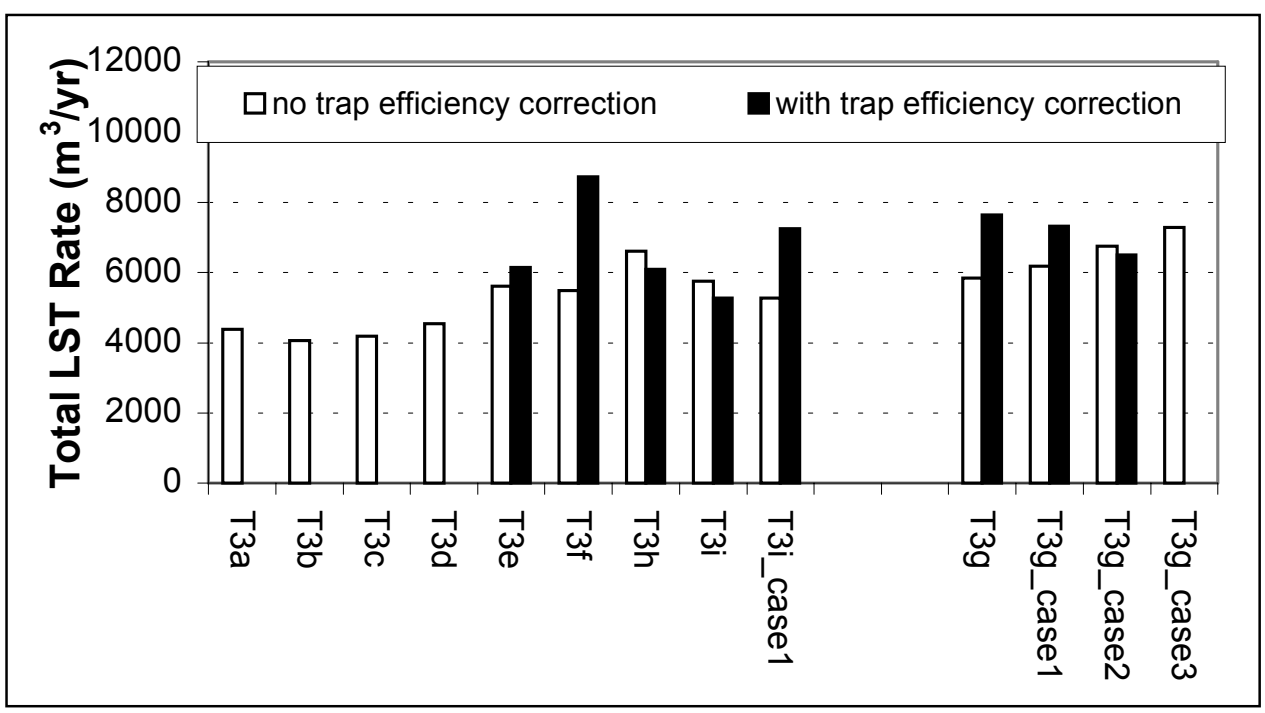

Figure 94. Total LST rates computed with and without corrections to account for anomalous volume change adjacent to the downdrift boundary for the plunging-breaker case

Results show that corrections made to compensate for anomalous beach change immediately adjacent to the downdrift boundary can be as much as 25 percent of the total LST rate. Another result is that the magnitude of the correction seems to be greatest just after the beach is remolded. The anomalies in beach bathymetry appear to develop quickly after the beach is remolded, and then with time, a near-equilibrium condition seems to develop in which the anomalies don't grow as fast, or grow very little. Note the lower magnitude of the correction prior to remolding that was done after T1h_case3, and the large correction immediately thereafter in T1h_case4 and T1h_case5. Also note the trend for the magnitude of the correction to decrease between the tests, T1h_case8 and T1h_case10, after the beach was remolded prior to T1h_case8.

A similar result was found for the plunging-breaker case (Figure 94). The beach was remolded prior to case $\mathrm{T} 3 \mathrm{~g}$, and with subsequent runs, the magnitude of the correction decreased. Again, the magnitude of the correction was as much as 25 percent of the total volume that accumulated in the traps. For run T3g_case3, malfunctions of the profiler precluded computation of corrections to the trap weights.

\section{Repeatability of LST Rates}

Results shown in Figures 93 and 94 also illustrate the degree of repeatability that can be expected in estimates of the total LST rate. Total transport rate estimates for the spilling breaker case ranged from 2,350 to $2,950 \mathrm{~m}^{3} / \mathrm{yr}$. Of the nine estimates made under what were essentially the same forcing conditions, six fell in the range from 2,600 to $2,850 \mathrm{~m}^{3} / \mathrm{yr}$. The mean value for all estimates is about $2,700 \mathrm{~m}^{3} / \mathrm{yr}$, which implies that estimates of total LST are repeatable, to within 10 to 12 percent, and more likely within about 4 to 6 percent for this wave condition. 
For the plunging-breaker case, total LST rates varied from 6,500 to $7,700 \mathrm{~m}^{3} / \mathrm{yr}$, with a mean of about $7,200 \mathrm{~m}^{3} / \mathrm{yr}$. Variability was similar, \pm 7 to 10 percent. Repeatability for the total LST rate is considered to be good.

Figures 95 and 96 show cross-shore distributions of corrected LST rates for both the spilling and plunging wave cases, respectively. In both figures, wave runs that should produce nearly the same transport rate distributions are shown. The general shape of the distribution was reproduced in all cases. The degree of repeatability in the distribution of LST also is considered to be good, but not quite as good as the total LST rate. At most locations, local estimates of LST generally vary by 10 to 25 percent about a mean value for all the wave runs. More variability is evident near the incipient breaker zone and in the zone closest to the shoreline.

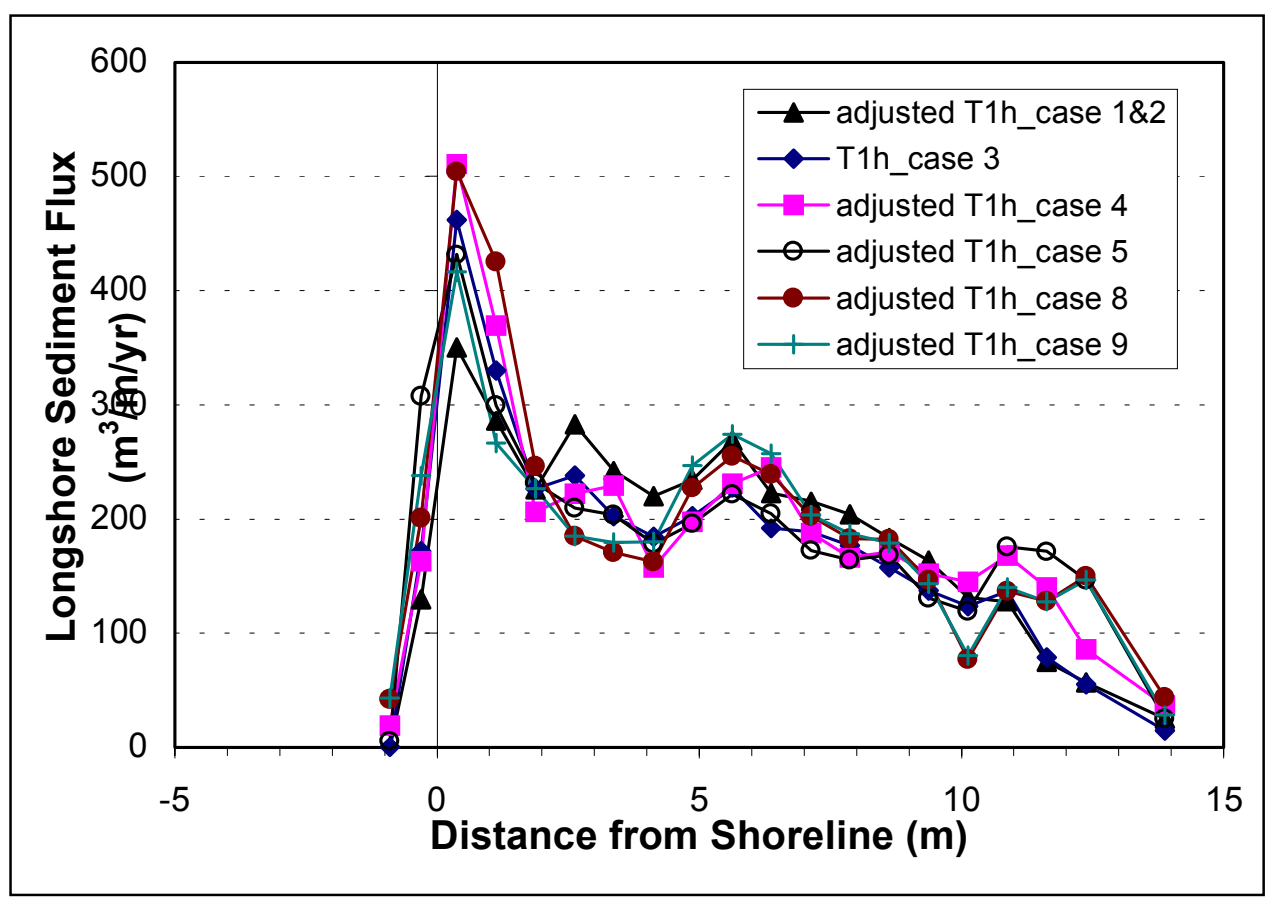

Figure 95. Cross-shore distribution of the LST rate for the spilling-breaker case

The variability of LST estimates in the nearshore zone is worth commenting on. Estimates in this region are considered to have the greatest degree of error and the lowest degree of repeatability, for a number of reasons. The foreshore zone is characterized by a relatively steep beach and is rather energetic as a result of the presence of the wave uprush and downrush. An adjustable brick template is used to hold the sand beach in place across the entire profile. Ceramic "pavers" having different thickness (1.4- and $2.8-\mathrm{cm})$ and standard construction bricks (5.6-cm thickness) are used to construct the template. The thinner pavers are used along most of the profile to provide better resolution for matching the natural beach elevations. In the energetic swash zone, the template is constructed mostly of standard-sized bricks so that they will not move when impacted by uprush bores. For both the spilling- and plunging-breaker cases, the pavers were not stable on the foreshore slope. Consequently the brick template is a rather crude approximation of the steep slope, and most importantly, edges of bricks are 


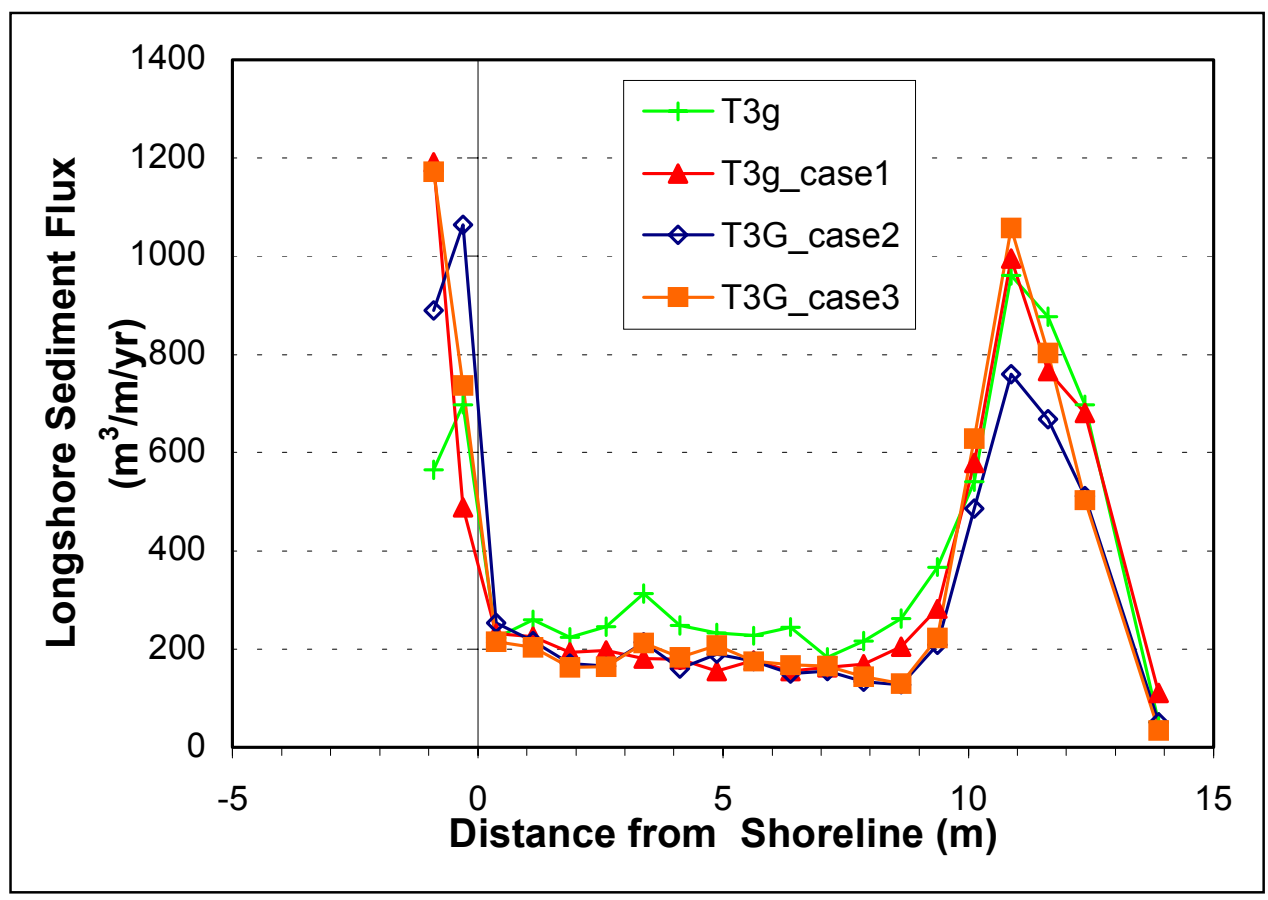

Figure 96. Cross-shore distribution of the LST rate, for the plunging-breaker case

exposed. Wave uprush interacts with the bricks and induces sediment suspension and transport. Depending on the sequencing and strength of uprush and downrush bores, sediment can be carried into any of the most landward traps. The distributions reflect considerable variability in sediment fluxes within the two or three most landward traps. The degree of variability is much more significant for the plunging wave case, presumably because the swash zone was much more energetic due to the longer wave periods. Corrections are made to trapped quantities in the swash zone to account for any anomalous erosion of the foreshore. 


\section{Conclusions}

A large-scale facility for investigating nearshore and surf zone sediment transport processes has been constructed and successfully applied. The facility provides the Corps of Engineers with a capability for studying longshore sand transport at scales that are much larger than the scales at which previous laboratory research has been conducted to examine these processes. The LSTF is state-of-the-art in terms of the systems that have been built for generating relatively large-scale laboratory waves (four phase-synchronized electric-motordriven wave generators), pumping and recirculating the longshore current (20 independent and separately controlled pump and piping networks for accurate control of the cross-shore distribution of the current), measuring the quantity of sand that moves along the beach (20 instrumented sediment traps), and a fully programmable instrumentation bridge and data acquisition system that includes 10 ADV current sensors, 10 capacitance-type wave gauges, four FOBS arrays, and an automated beach profiling system. All facility components were designed to maximize accuracy of data acquired in movable-bed experiments and minimize the amount of human labor (and time) required to perform them.

Two comprehensive test series were conducted on an planar concrete beach, one involving regular waves and the other irregular waves. These data are valuable for several reasons: (a) the geometric scale of the experiments is significantly larger than the scale of laboratory experiments conducted in the past; (b) the concrete beach has a relatively mild 1:30 slope, unlike previous laboratory experiments; (c) both regular and irregular wave forcing were used; and (d) the vertical velocity structure of the longshore current was accurately measured using ADVs. These data are provided for use in theoretical studies and for calibration and verification of analytical and numerical models.

The pump settings that produced the proper longshore current distribution in the LSTF were determined through an iterative process based on mean longshore current measurements in the surf zone and offshore in the internal recirculation zone. This procedure worked well. When the proper distribution is pumped, the degree of longshore uniformity in mean longshore current increases noticeably, and the magnitude of the current offshore in the recirculation zone approaches a minimum value. Internal recirculation cannot be completely eliminated, however, and its magnitude was about 10 percent of the total flow rate in the surf zone for the two wave cases examined on the concrete beach.

The final selection of proper pump settings was confirmed by the insightful criteria developed by Visser (1991). But unlike the findings of Visser for the 
facility he used, for the LSTF, $Q_{p}$ can vary by as much as \pm 20 percent of $Q_{p u}$ without a significant increase in $\mathrm{Q}_{\mathrm{r}}$. For this reason, emphasis during the iterative process is placed on examination of the measured data, taking advantage of the high degree of cross-shore resolution in the recirculation system itself. However, even for this range of $Q_{p}$ the inshore two-thirds of the mean longshore current distribution was relatively unaffected by $\mathrm{Q}_{\mathrm{r}}$. The effect of internal recirculation, $\mathrm{Q}_{\mathrm{r}}$ manifested itself primarily on the offshore tail of the measured longshore current distribution. Therefore, tuning of the longshore current focused on the inshore portion of the distribution first, saving the offshore tail for last.

Measured currents in the offshore tail were always higher than the equivalent pump settings, regardless of the magnitude because of the internal recirculation in the region offshore of the surf zone.

The active external longshore current recirculation system and the operational procedure used to pump the proper current, led to a $12-\mathrm{m}$ long region of the beach that was characterized by a very high degree of longshore uniformity in waves, currents, and mean water levels, between Y (longshore) coordinates of 19 and $31 \mathrm{~m}$. The standard deviation of the longshore variation in longshore current is still relatively small downstream to $\mathrm{Y}=14 \mathrm{~m}$, at the downdrift boundary.

Two experiments that involved a movable-bed quartz-sand beach, which had a very narrow distribution of grain sizes and a median grain diameter of 0.15 $\mathrm{mm}$, were successfully conducted. Irregular wave conditions were generated in each experiment. The first involved wave conditions that produced predominantly spilling-type wave breaking. In the second experiment predominantly plunging breakers were generated. For both experiments, waves having a similar significant wave height were adopted. The type of wave breaking was controlled by use of a different wave period.

Each experiment was comprised of a series of tests, or runs. Initial tests were conducted to iterate toward the proper longshore current distribution in the presence of a movable bed and to allow the beach profile shape to reach an equilibrium, or near-equilibrium, condition. Iterative procedures that were determined from the concrete-beach experiments were successfully applied to the movable-bed experiments. Subsequent wave runs involved acquisition of data to examine: alongshore variability in processes, steadiness, and repeatability of measurements and estimates of local and total LST rates, and investigate the vertical structure of currents and sediment concentration.

Results suggest that breaker-type and beach morphology exert a strong influence on the total LST rate and its cross-shore distribution. More LST occurred for the plunging wave case than for the spilling wave case, despite both cases having approximately the same incident wave height and direction (the former LST rate was 2.6 to 2.7 times greater than the latter). The cross-shore distribution of LST for each case was different. For the spilling-breaker case, the magnitude of the LST rate gradually increased with increasing proximity to the shoreline, and a local peak in the distribution existed in the swash zone. For the plunging-breaker case, a local peak in the LST distribution existed at the breakpoint bar that was produced. A second peak existed in the swash zone, as was found for the spilling-breaker case, but the magnitude of the swash zone 
transport was much higher as a result of the steeper beach slope and much more energetic swash zone that developed for waves having longer periods. In the midsurf zone, the local rates of LST were similar for the two wave cases.

As was found for the concrete-beach experiments, there was a region of the movable-bed beach that was characterized by a reasonably high degree of alongshore uniformity in waves, currents, and sediment concentration, between $Y$ (alongshore) coordinates of 15 and $30 \mathrm{~m}$. The downdrift boundary is not perfect though, and imperfections lead to sand trap efficiencies that are not 100 percent. Slight accretion or erosion anomalies developed adjacent to the downdrift boundary, and these anomalies must be accounted for in deriving estimates of LST rates. Accurate and dense survey data are required to compute the corrections. Errors associated with sand bypassing the traps were rather small.

The steadiness of mean flow conditions, quality of the sensors, and repeatability of the measurements allow accurate data sets to be acquired, not only of wave, current (at one depth), and water elevation parameters, but also the vertical structure of the mean current and sediment concentration fields.

Sediment concentration measurements were more variable than wave and current measurements, probably because of the small differences in local morphology that exist throughout the beach. Mean values computed from several adjacent transects are recommended for characterizing the vertical structure of sediment concentration. Overall, steadiness and repeatability of the hydrodynamic conditions allowed high-quality data sets of suspended sand concentrations and longshore sediment transport rates to be acquired.

The LSTF has proven to be an excellent and robust facility, and so far has yielded unprecedented measurements of surf zone sediment transport processes in a laboratory setting, including sand transported in suspension. The potential for making $R \& D$ advancements through the use of the facility is quite high. 


\section{References}

Allen-Bradley, Inc. (1995). User manual for serial communications module for adjustable frequency AC drives. Publication 1203, Rockwell Automation, Milwaukee, WI.

. (1996). User manual for adjustable frequency AC drives.

Publication 1336, Rockwell Automation, Milwaukee, WI.

Brebner, A., and Kamphuis, J. W. (1963). "Model tests on the relationship between deep-water wave characteristics and longshore currents," Civil Eng. Res. Rep. 31, Queen's University, Kingston, Canada.

Dalrymple, R. A., and Dean, R. G. (1972). "The spiral wavemaker for littoral drift studies." Proc. 13th Int. Conf. Coastal Eng. Vancouver, Canada, 689705 .

Data Industrial. (1994). “Owner's manual for series 200 non-magnetic impeller flow sensors," Publication PN 7283, Data Industrial Corporation, Mattapoisett, MA.

Dean, R. G. (1977). "Equilibrium beach profiles: U.S. Atlantic and Gulf coasts," Ocean Engineering Report No. 12, Department of Civil Engineering, University of Delaware, Newark, DE.

Delft Hydraulics. (1998). User's manual for PV-12 profile indicator. Delft Hydraulics, Rotterdamseweg, The Netherlands.

Dominis Engineering Ltd. (1992). "GPLOT user's guide," Dominis Engineering Ltd., Gloucester, Ontario, Canada.

Galvin, C. J., and Eagleson, P. S. (1965). "Experimental study of longshore currents on a plane beach," U.S. Army Engineer Waterways Experiment Station, Tech. Memo No. 10, Vicksburg, MS.

Hamilton, D. G., and Ebersole, B. A. (2001). "Establishing uniform longshore currents in a large-scale sediment transport facility," Coastal Engineering 42, 199-218.

Hamilton, D. G., Neilans, P. J., Rosati, J. D., Fowler, J. E., and Smith, J. M. (1997). "Hydraulic design of a large-scale longshore current recirculation 
system," Proc. Coastal Dynamics '97, Plymouth, United Kingdom. American Society of Civil Engineers, 516-525.

Hamilton, D. G., Rosati, J. D., Fowler, J. E., and Smith, J. M. (1996). "Design capacity of a longshore current recirculation system for a longshore sediment transport laboratory facility." Proc. 25th Int. Conf. Coastal Eng. Orlando, FL, American Society of Civil Engineers, 3,628-3,641.

HR Wallingford. (1994). "Understanding the nearshore environment." A publication describing the coastal research facility at H.R. Wallingford, Wallingford, England.

Hughes, S. A. (1993). Physical models and laboratory techniques in coastal engineering. World Scientific, Singapore.

Kamphuis, J. W. (1977). "Discussion on wave-induced circulation in shallow basins," J. Waterway, Port, Coastal Ocean Div., 103, 570-571.

Kraus, N. C., and Larson, M. (1991). "NMLONG: Numerical model for simulating the longshore current, Report 1: Model development and tests,", Tech. Report DRP-91-1, U.S. Army Engineer Waterways Experiment Station, Vicksburg, MS.

Markle, D. G., and Greer, H. C. (1992). "Crescent city instrumented model dolos study; coastal model investigation," U.S. Army Engineer Waterways Experiment Station, Tech. Report CERC-92-15, Vicksburg, MS.

Miles, M. D. (1997). “GEDAP user's guide for Windows NT," Canadian Hydraulics Centre, National Research Council of Canada, Technical Report, HYD-TR-021.

Mizuguchi, M., and Horikawa, K. (1978). "Experimental study on longshore current velocity distribution," Fac. Sci. Eng., Chuo Univ., Tokyo, Japan, Bull. 21, 123-150.

MTS Systems Corporation. (1998). "Operation manual and assembly drawings for the repositionable spectral wave generators and instrumentation bridge," Vol 1 and 2, Eden Prairie, MN.

Putnam, J. A., Munk, W. H., and Traylor, M. A. (1949). "The prediction of longshore currents," Trans. Am. Geophys. Union, 30, 337-345.

Putrevu, U., and Svendsen, I. A. (1992). "A mixing mechanism in the nearshore region." Proc. 23rd Int. Conf. Coastal Eng, Venice, Italy, American Society of Civil Engineers, 2,758-2,771.

Putrevu, U., Oltman-Shay, J., and Svendsen, I. A. (1995). "Effects of alongshore nonuniformities on longshore current predictions," J. Geophys. Res. 100, 16,119-16,130. 
Rosati, J. D., and Kraus, N. C. (1989). "Development of a portable sand trap for use in the nearshore," U.S. Army Engineer Waterways Experiment Station, Tech. Report CERC-89-11, Vicksburg, MS.

Rosati, J. D., Hamilton, D. G., Fowler, J. E., and Smith, J. M. (1995). “Design of a laboratory facility for longshore sediment transport research." Proc.

Coastal Dynamic '95, Gdansk, Poland. American Society of Civil Engineers, 771-782.

Shore Protection Manual. (1984). 4th ed., 2 Vol, U.S. Army Engineer Waterways Experiment Station, U.S. Government Printing Office, Washington, DC.

Simons, R. R., Whitehouse, R. J. S., MacIver, R. D., Pearson, J., Sayers, P. B., Zhao, Y., and Channell, A. R. (1995). "Evaluation of the UK Coastal Research Facility." Proc. Coastal Dynamics '95, Gdansk, Poland. American Society of Civil Engineers, 161-172.

SonTek. (1997). "Operation and reference manual for acoustic doppler velocitimeter," SonTek, Inc., San Diego, CA.

Sutherland, J., Channell, A. R., and Whitehouse, R. J. S. (1999). "Design and evaluation of a sediment recirculation system for a coastal wave basin," UK Coastal Research Facility. HR Wallingford, Report TR-97.

Svendsen, I. A. (1991). "Development of a comprehensive plan for modeling longshore current generation in the laboratory," Unpublished Report for the U.S. Army Engineer Waterways Experiment Station, Vicksburg, MS.

U.S. Motors. (1996). "Reference manual for drive motors on vertical turbine pumps," Publication PB 500, U.S. Electric Motors Division, Emerson Electric Co., St. Louis, MO.

Visser, P. J. (1982). "The proper longshore current in a wave basin," Comm. on Hyd. Rep. 82-1, Dept. of Civil Eng. Delft Univ. of Technology, Delft, The Netherlands.

. (1991). "Laboratory measurements of uniform longshore currents," Coastal Eng. 15, 563-593. 


\section{Appendix A Hydrodynamic Data from Fixed-bed Experiments}

The primary data sets for Test $6 \mathrm{~N}$ (regular waves) and Test $8 \mathrm{E}$ (irregular waves) are provided in tabular form in Table A-1 and Table A-2, respectively. Each table lists the measured mean longshore current at transects Y19, Y23, Y27, and $\mathrm{Y} 31$, as well as the longshore-averaged value for these four transects, $\mathrm{V}_{\text {avg; }}$; the still-water depth, d; the longshore-averaged value of wave setup or setdown, $\eta_{\text {avg, }}$ and wave height, $H_{\text {avg. }}$. The energy-based significant wave height, listed for Test $8 \mathrm{E}$, is based on using a lower cutoff frequency of $0.2 \mathrm{~Hz}$ (i.e., $0.5 \times \mathrm{F}_{\mathrm{p}}$ ), to remove the low-frequency energy from the water surface elevation time series. The cross-shore (x-axis) positions of the measurements are listed in the standard basin coordinates. The still-water depth can be calculated as

$$
\begin{aligned}
& d=x / 30-0.1, \text { for } 3.0 m \leq x \leq 18.0 m \\
& d=x / 18-0.5, \text { for } 18.0 m \leq x \leq 21.0 m \\
& d=0.667 m, \text { for } x \geq 21.0 m
\end{aligned}
$$

\begin{tabular}{|c|c|c|c|c|c|c|c|c|}
\hline \multicolumn{9}{|c|}{$\begin{array}{l}\text { Table A1 } \\
\text { Primary Data Set from Regular Wave Test 6N }\end{array}$} \\
\hline $\begin{array}{l}\text { X-Loc. } \\
m\end{array}$ & $\begin{array}{l}\mathrm{Y19} \\
\mathrm{m} / \mathrm{s}\end{array}$ & $\begin{array}{l}\mathrm{Y23} \\
\mathrm{m} / \mathrm{s}\end{array}$ & $\begin{array}{l}\mathrm{Y} 27 \\
\mathrm{~m} / \mathrm{s}\end{array}$ & $\begin{array}{l}31 \\
\mathrm{~m} / \mathrm{s}\end{array}$ & $\begin{array}{l}\mathrm{V}_{\text {avg }} \\
\mathrm{m} / \mathrm{s}\end{array}$ & $\begin{array}{l}d \\
m\end{array}$ & $\begin{array}{l}\eta_{\text {avg }} \\
\mathrm{m}\end{array}$ & $\begin{array}{l}\mathrm{H}_{\text {avg }} \\
\mathrm{m}\end{array}$ \\
\hline 4.12 & 0.297 & 0.338 & 0.302 & $\begin{array}{l}0.188 \\
\end{array}$ & $\overline{c 0.281}$ & 0.037 & 0.025 & 0.047 \\
\hline 5.72 & 0.401 & 0.422 & 0.392 & 0.397 & 0.403 & 0.091 & 0.020 & 0.077 \\
\hline 7.12 & 0.417 & 0.421 & 0.426 & 0.434 & 0.424 & 0.137 & 0.012 & 0.101 \\
\hline 8.62 & 0.368 & 0.392 & 0.390 & 0.342 & 0.373 & 0.187 & 0.001 & 0.131 \\
\hline 10.12 & 0.323 & 0.301 & 0.305 & 0.312 & 0.3210 & 0.237 & -0.006 & 0.190 \\
\hline 11.52 & 0.173 & 0.145 & 0.154 & 0.169 & 0.160 & 0.284 & -0.010 & 0.254 \\
\hline 13.12 & 0.060 & 0.058 & 0.075 & 0.090 & 0.071 & 0.337 & -0.013 & 0.235 \\
\hline 14.62 & 0.016 & -0.005 & 0.031 & 0.046 & 0.022 & 0.387 & -0.012 & 0.209 \\
\hline 15.62 & -- & -- & -0.007 & -- & -0.007 & 0.421 & -- & -- \\
\hline 16.12 & -- & -- & -- & -- & -- & 0.437 & -0.013 & 0.206 \\
\hline 16.85 & -- & -- & -- & -- & -- & 0.462 & -0.010 & 0.203 \\
\hline 18.00 & -- & -- & -- & -- & -- & 0.500 & -0.011 & 0.190 \\
\hline
\end{tabular}




\begin{tabular}{|c|c|c|c|c|c|c|c|c|}
\hline \multicolumn{9}{|c|}{$\begin{array}{l}\text { Table A2 } \\
\text { Primary Data Set from Irregular Wave Test 8E }\end{array}$} \\
\hline $\begin{array}{l}\text { X-Loc. } \\
m\end{array}$ & $\begin{array}{l}\mathrm{Y} 19 \\
\mathrm{~m} / \mathrm{s}\end{array}$ & $\begin{array}{l}\mathrm{Y} 23 \\
\mathrm{~m} / \mathrm{s}\end{array}$ & $\begin{array}{l}\mathrm{Y} 27 \\
\mathrm{~m} / \mathrm{s}\end{array}$ & $\begin{array}{l}31 \\
\mathrm{~m} / \mathrm{s}\end{array}$ & $\begin{array}{l}\begin{array}{l}V_{\text {avg }} \\
\mathrm{m} / \mathrm{s}\end{array} \\
\end{array}$ & $\begin{array}{l}d \\
m\end{array}$ & $\begin{array}{l}\eta_{\text {avg }} \\
\mathrm{m}\end{array}$ & $\begin{array}{l}\begin{array}{l}H_{\text {mo-avg }} \\
m\end{array} \\
\end{array}$ \\
\hline 4.12 & 0.188 & 0.227 & 0.207 & 0.154 & 0.194 & 0.037 & 0.016 & 0.053 \\
\hline 5.72 & 0.303 & 0.326 & 0.316 & 0.306 & 0.313 & 0.091 & 0.013 & 0.082 \\
\hline 7.12 & 0.326 & 0.341 & 0.333 & 0.346 & 0.337 & 0.137 & 0.006 & 0.107 \\
\hline 8.62 & 0.297 & 0.313 & 0.298 & 0.309 & 0.304 & 0.187 & 0.001 & 0.138 \\
\hline 10.12 & 0.270 & 0.277 & 0.267 & 0.267 & 0.270 & 0.237 & -0.001 & 0.167 \\
\hline 11.52 & 0.228 & 0.233 & 0.226 & 0.221 & 0.227 & 0.284 & -0.004 & 0.185 \\
\hline 13.12 & -- & -- & -- & -- & -- & 0.337 & -0.008 & 0.206 \\
\hline 13.88 & 0.122 & 0.128 & 0.121 & 0.125 & 0.124 & 0.363 & -- & -- \\
\hline 14.62 & -- & -- & -- & -- & -- & 0.387 & -0.009 & 0.214 \\
\hline 16.12 & 0.038 & 0.012 & 0.010 & 0.031 & 0.023 & 0.437 & -0.010 & 0.224 \\
\hline 16.85 & -- & -- & -- & -- & -- & 0.462 & -0.008 & 0.227 \\
\hline 18.00 & -- & -- & -- & -- & -- & 0.500 & -0.007 & 0.232 \\
\hline
\end{tabular}

Table A-3 provides a number of other measured and calculated wave and water level quantities in the surf zone, where $h$ is the longshore-averaged mean water depth (equal to $d+\eta$ ), $\eta$ is the mean water surface elevation, $\eta_{m}$ is the maximum value of wave setup, estimated by linear extrapolation, $\gamma$ is the surf zone averaged value of $\mathrm{H} / \mathrm{h}$, and the subscript, "br", refers to values at the breaker line. The breaking wave angle, $\theta_{\mathrm{br}}$, was estimated using Snell's law.

For the regular wave case, the breaking point is assumed to be the point where the measured wave height reaches its maximum value. For the irregular wave case, the breaking point was assumed to be the point where the measured significant wave height began to decrease at the highest rate (Figure 68). This occurred at the location $\mathrm{x}=13.1 \mathrm{~m}$, where the longshore-averaged significant wave height was $0.21 \mathrm{~m}$.

Estimates of the position of the mean waterline are $\mathrm{x}=2.1 \mathrm{~m}$ for the regular wave case and $x=2.4 \mathrm{~m}$ for the irregular wave case. It should be noted that the cross-shore position of the mean waterline could only be estimated with an accuracy of approximately $\pm 0.1 \mathrm{~m}$. Therefore, the estimates of maximum wave setup are only accurate to within $\pm 0.003 \mathrm{~m}$.

\begin{tabular}{|c|c|c|c|c|c|c|c|c|c|}
\hline \multicolumn{10}{|c|}{$\begin{array}{l}\text { Table A3 } \\
\text { Summary of Wave and Water Level Conditions in the Surf Zone }\end{array}$} \\
\hline Test & $\begin{array}{l}\mathrm{H}_{\mathrm{br}} \\
\mathbf{m}\end{array}$ & $\begin{array}{l}h_{b r} \\
m\end{array}$ & $\underline{H}_{b r} / h_{b r}$ & $\begin{array}{l}Y \\
-\end{array}$ & $\begin{array}{l}\Theta_{\mathrm{br}} \\
\text { deg }\end{array}$ & $\begin{array}{l}X_{\mathrm{br}} \\
\mathrm{m}\end{array}$ & $\begin{array}{l}\eta_{m} \\
m\end{array}$ & $\begin{array}{l}X-\text { Loc. } \\
\text { at } \eta_{m} \\
m\end{array}$ & $\begin{array}{l}\text { X-Loc } \\
\text { at SWL } \\
\mathrm{m}\end{array}$ \\
\hline 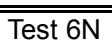 & 0.254 & 0.274 & 0.93 & $\overline{0.74}$ & "6.7 & $\bar{~} 111.5$ & 0.033 & 2.1 & 3.00 \\
\hline Test 8E & 0.206 & 0.329 & 0.63 & 0.75 & 7.3 & 13.1 & 0.021 & 2.4 & 3.00 \\
\hline
\end{tabular}

Figure A-1 shows the cross-shore variation in the vertical structure of the mean longshore current for the regular wave case (Test 6N). In general, the mean longshore current is rather uniform with depth. In the innersurf zone (ADV 1 through ADV 5), there is a slight increase in current speed with distance from the bed, and outside the surf zone (ADV 7 and 8) a slight decrease in current speed with distance from the bed. Visser $(1991)^{1}$ measured similar trends in the vertical velocity structure using regular waves. Putrevu and Svendsen

\footnotetext{
${ }^{1}$ References are listed in section following main text.
} 
(1992) presented a theoretical model that predicts similar trends in the vertical velocity structure. ADV 6 was located at the position of incipient wave breaking.

Figure A-2 shows the vertical structure of the mean longshore current for the irregular wave case (Test $8 \mathrm{E}$ ). The mean longshore current in this case is also rather uniform with depth. In the innersurf zone (ADV 1 through ADV 6), there is a slight increase in current speed with distance from the bed, and the vertical variations are similar to those measured for the regular wave case. In the outer surf zone (ADV 7 and 8), the mean velocity is relatively invariant with depth. This trend is different from that in the regular wave case, where the mean velocity decreased slightly with increasing distance from the bottom. However, in the regular wave case, no waves were breaking at ADV 7 and 8; whereas in the irregular wave case, some wave breaking occurred in this region.

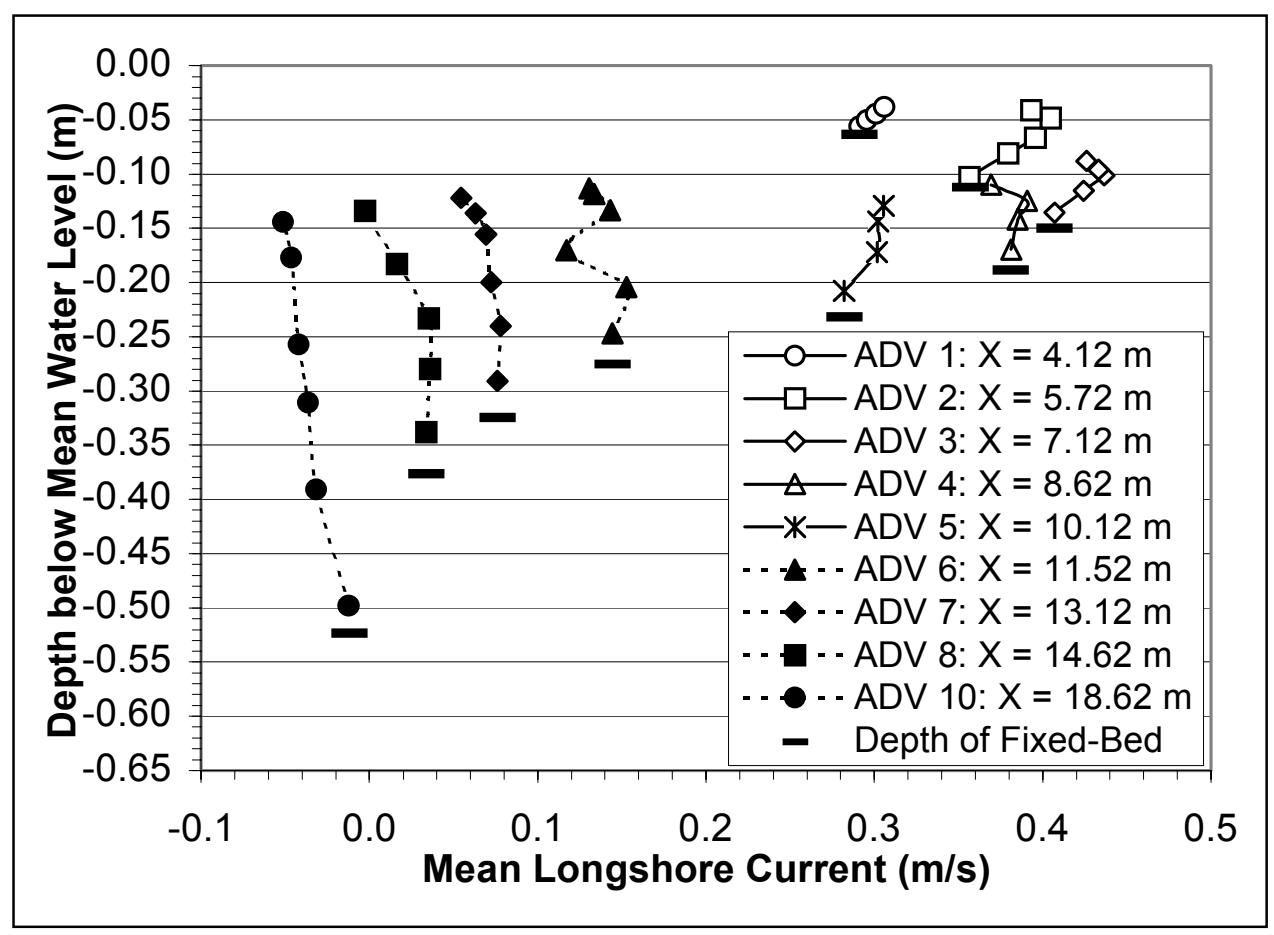

Figure A1. Test $6 \mathrm{~N}$ : Vertical structure of the mean longshore current at Y27 


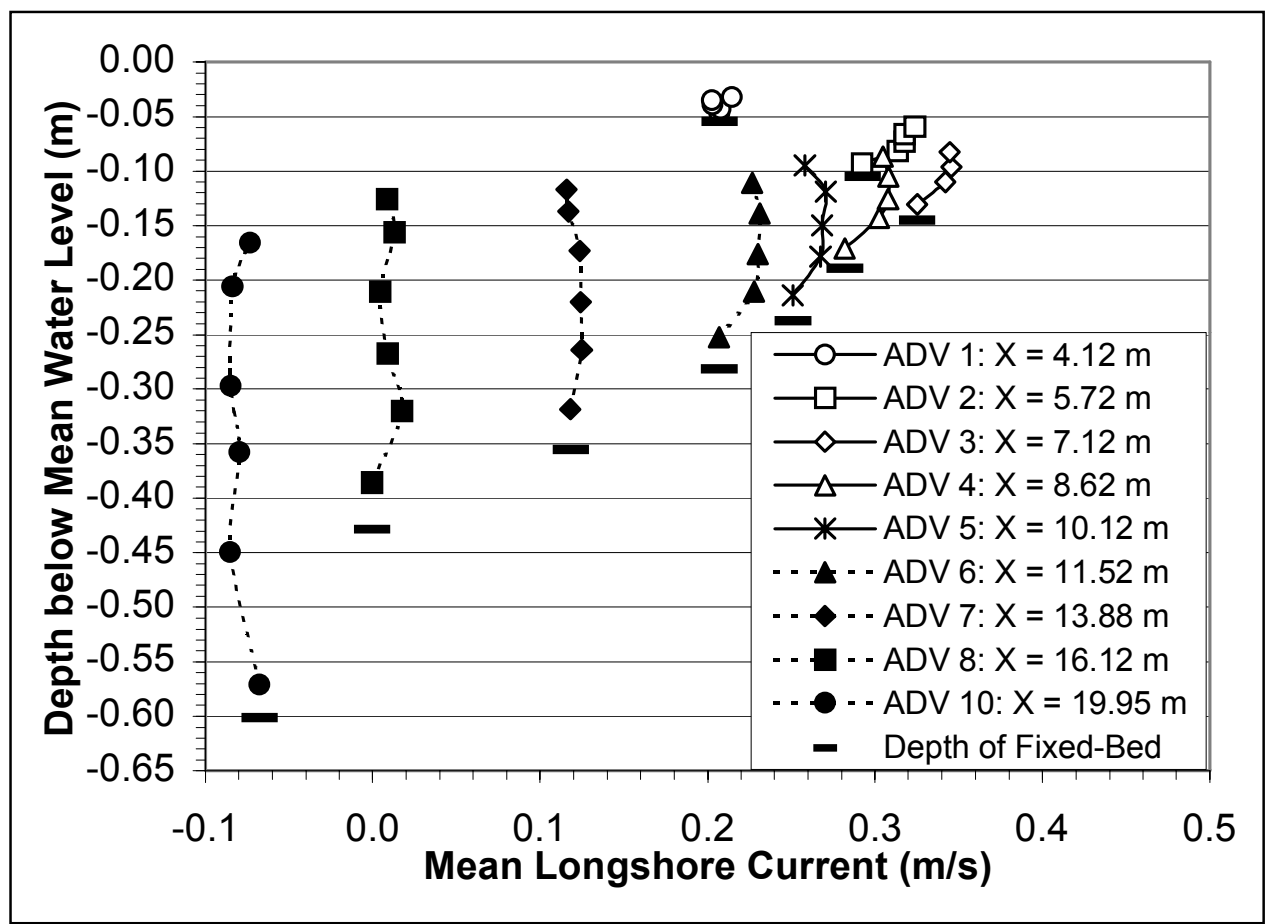

Figure A2. Test 8E: Vertical structure of the mean longshore current at Y27 


\section{Appendix B Photographs of the Large- scale Sediment Transport Facility}

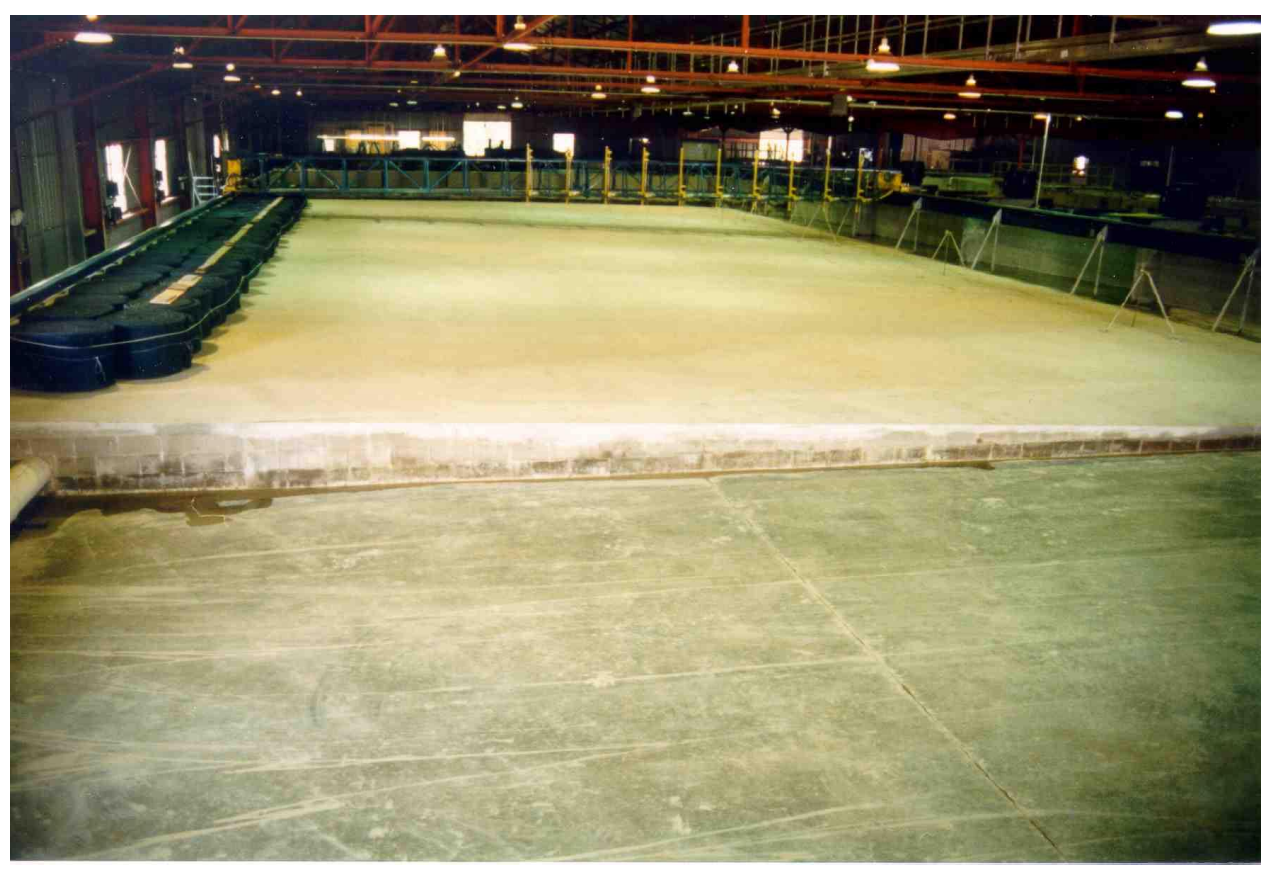

Figure B1. The concrete beach has a 1:30 slope. The beach was constructed as far updrift as possible so that the sand falls into the traps before reaching the pumps used to recirculate the longshore current 


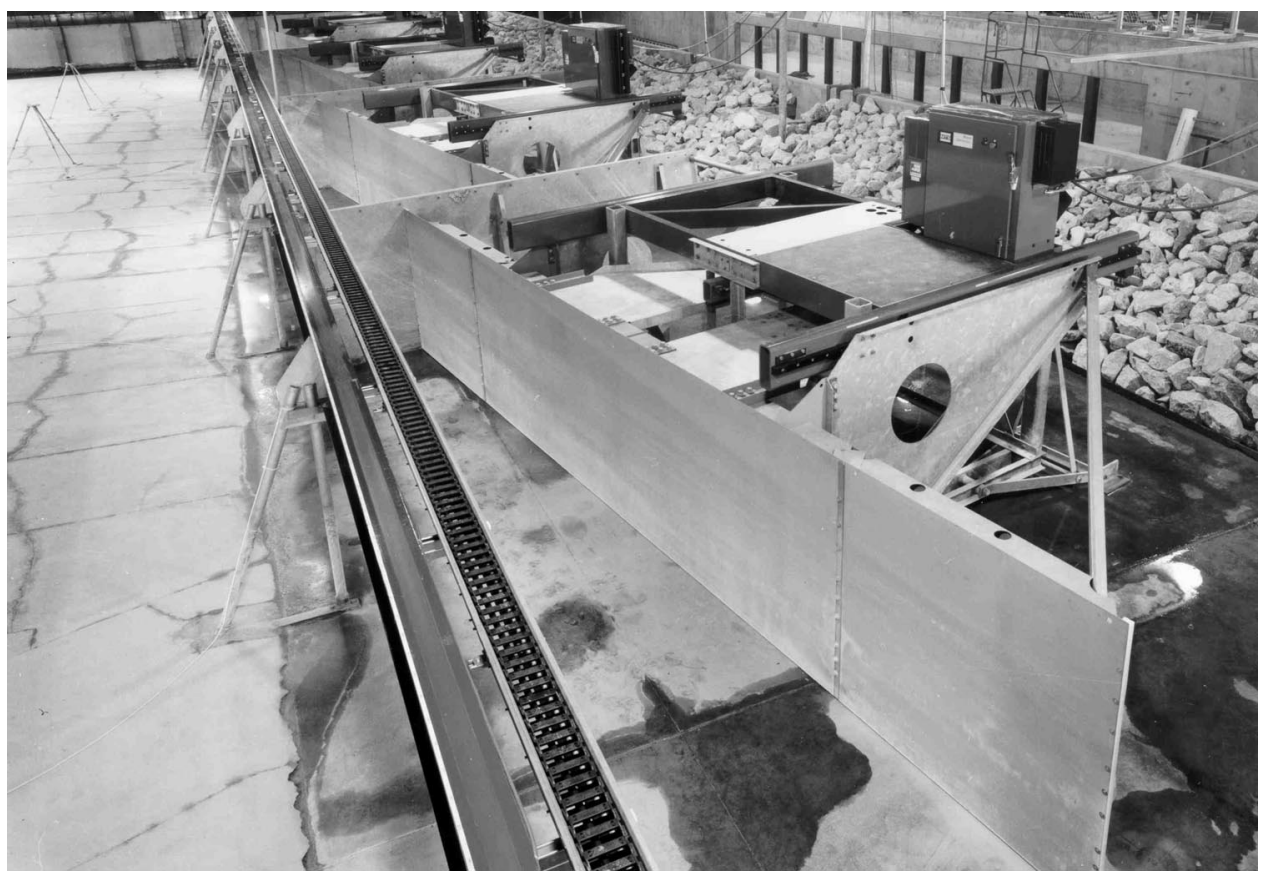

Figure B2. Four piston-type wave generators are shown with the facility drained. Each wave board is $7.62 \mathrm{~m}$ long and is oriented at $10 \mathrm{deg}$ to shorenormal in this figure. The wave generators can be positioned within the range of 0 through 20 deg relative to shore-normal

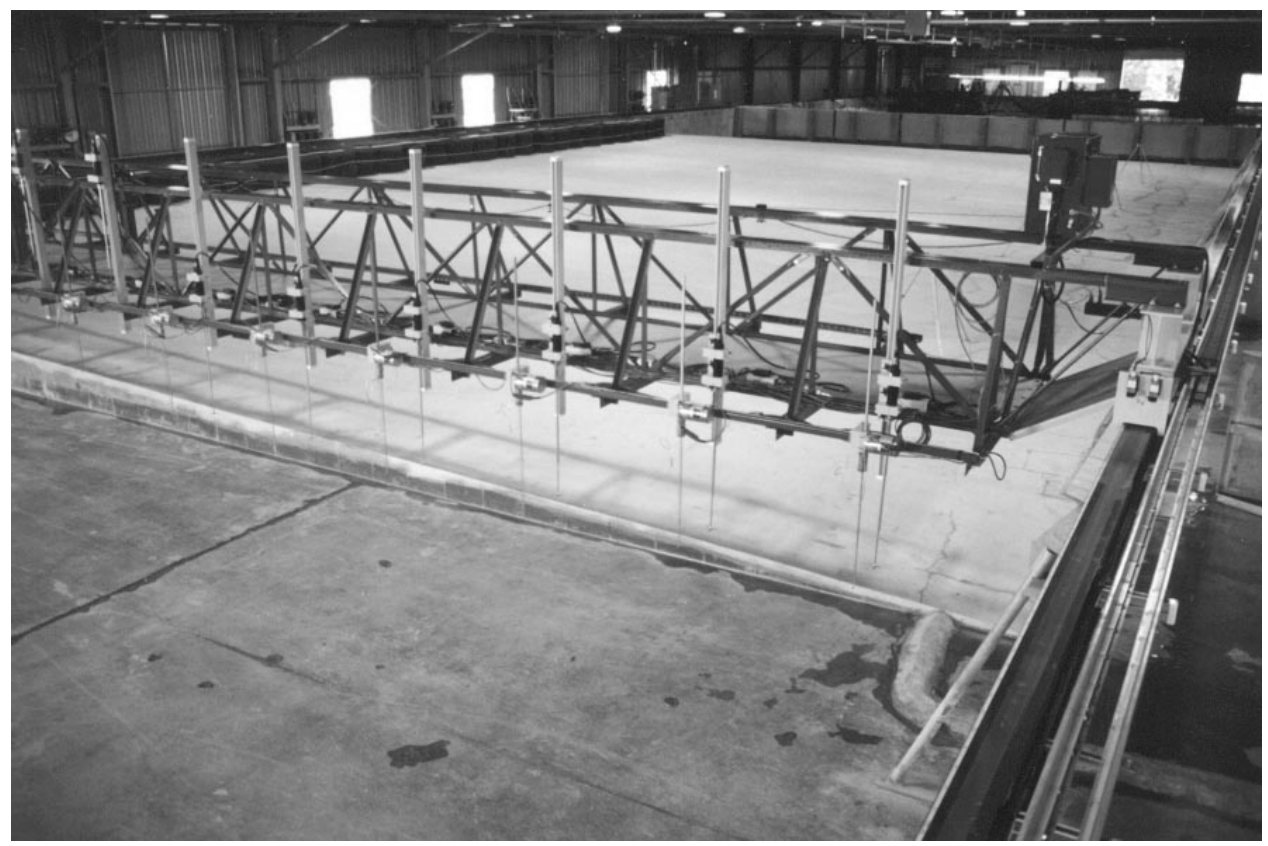

Figure B3. The instrumentation bridge is $21 \mathrm{~m}$ long and spans the entire width of the beach. The offshore support rail is located at the far right in this photograph. The bridge is designed to support wave gauges, current meters, optical backscatter sensors, and an automated beach profile indicator. The bridge also functions as an observation platform 


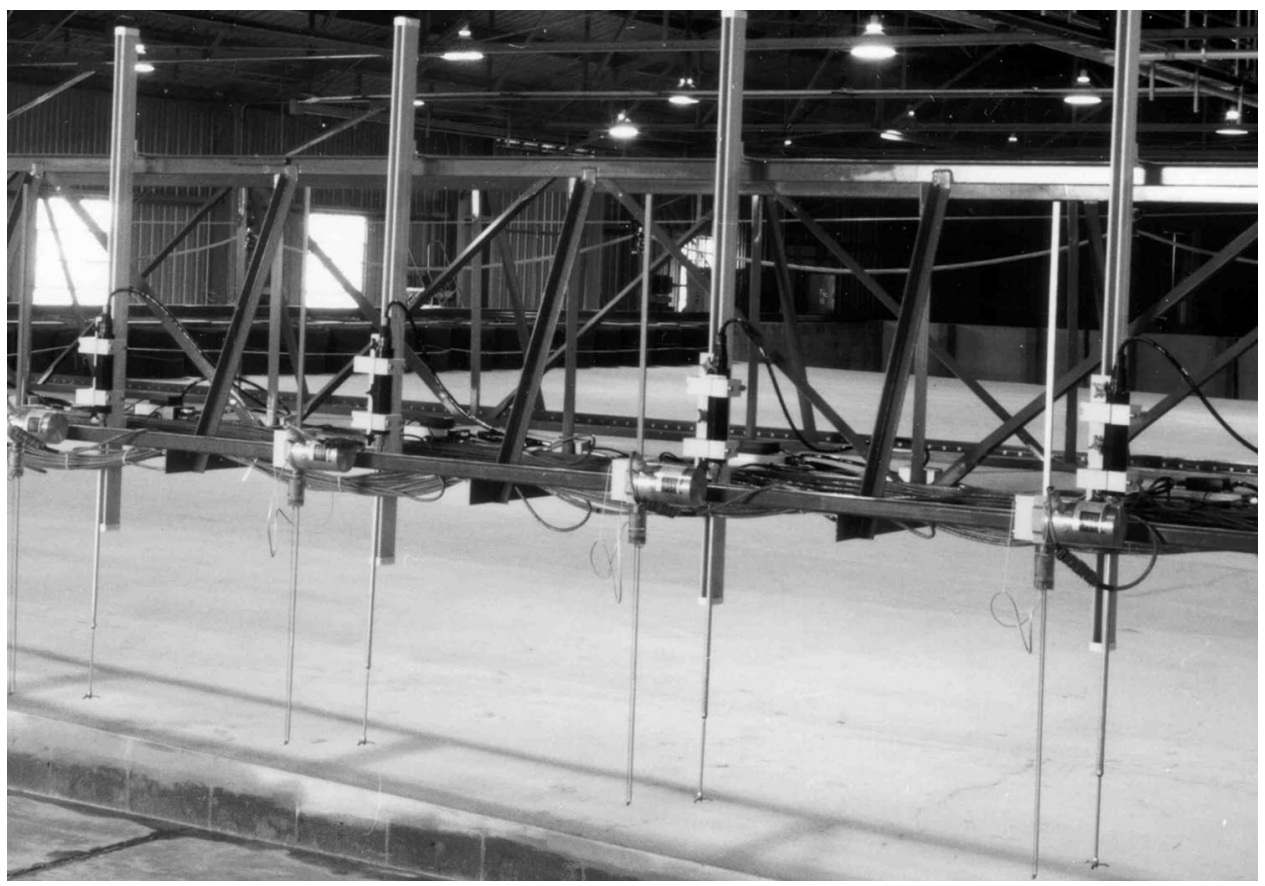

Figure B4. Wave gauges and current meters are co-located at various distributions across the width of the surf zone. The height and orientation of the current meters can be readily adjusted using the vertical mounting mechanisms shown in the figure

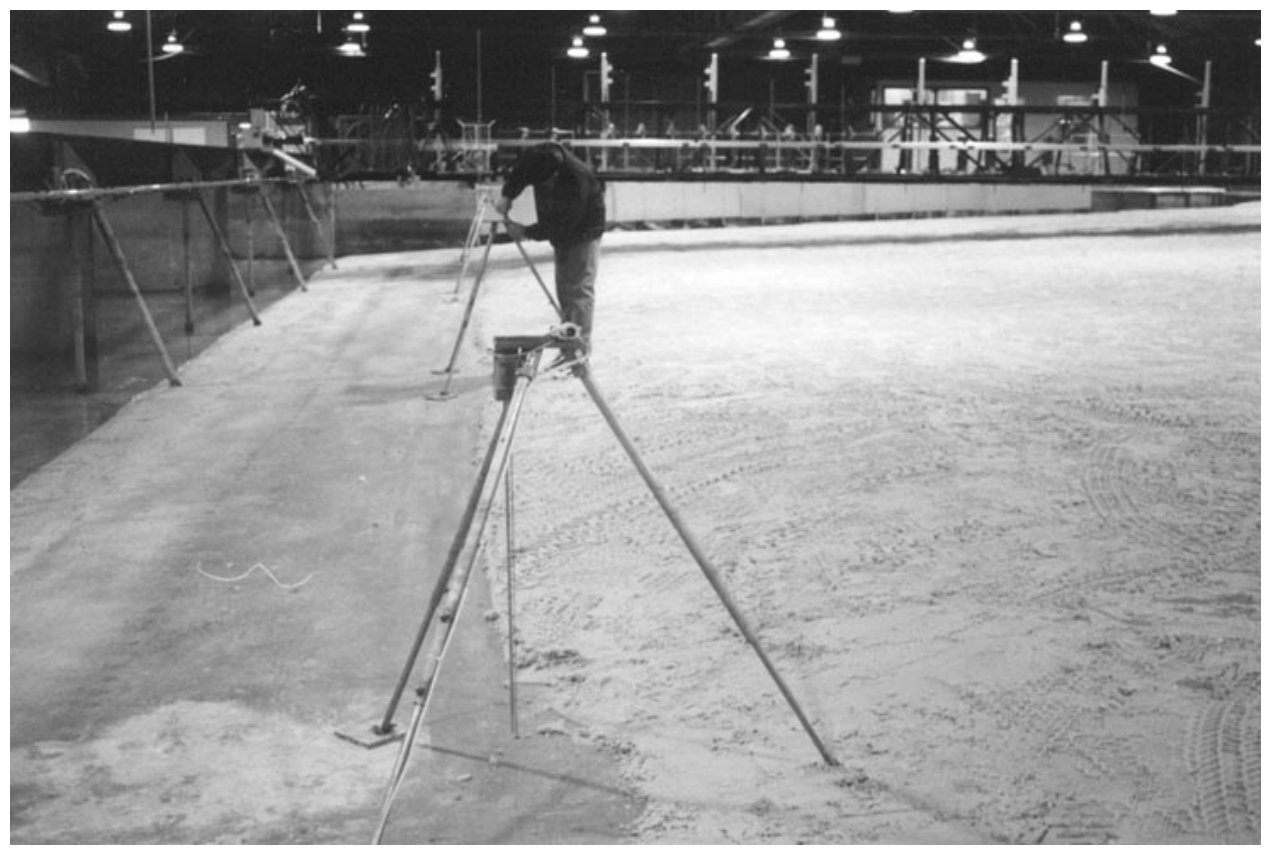

Figure B5. The offshore wave gauges are mounted to stainless steel tripods along the toe of the sand beach. There are four offshore wave gauges, one centered in front of each wave generator. This photograph is viewed from upstream. An electronics technician is inspecting one of the wave gauges while the facility is drained 


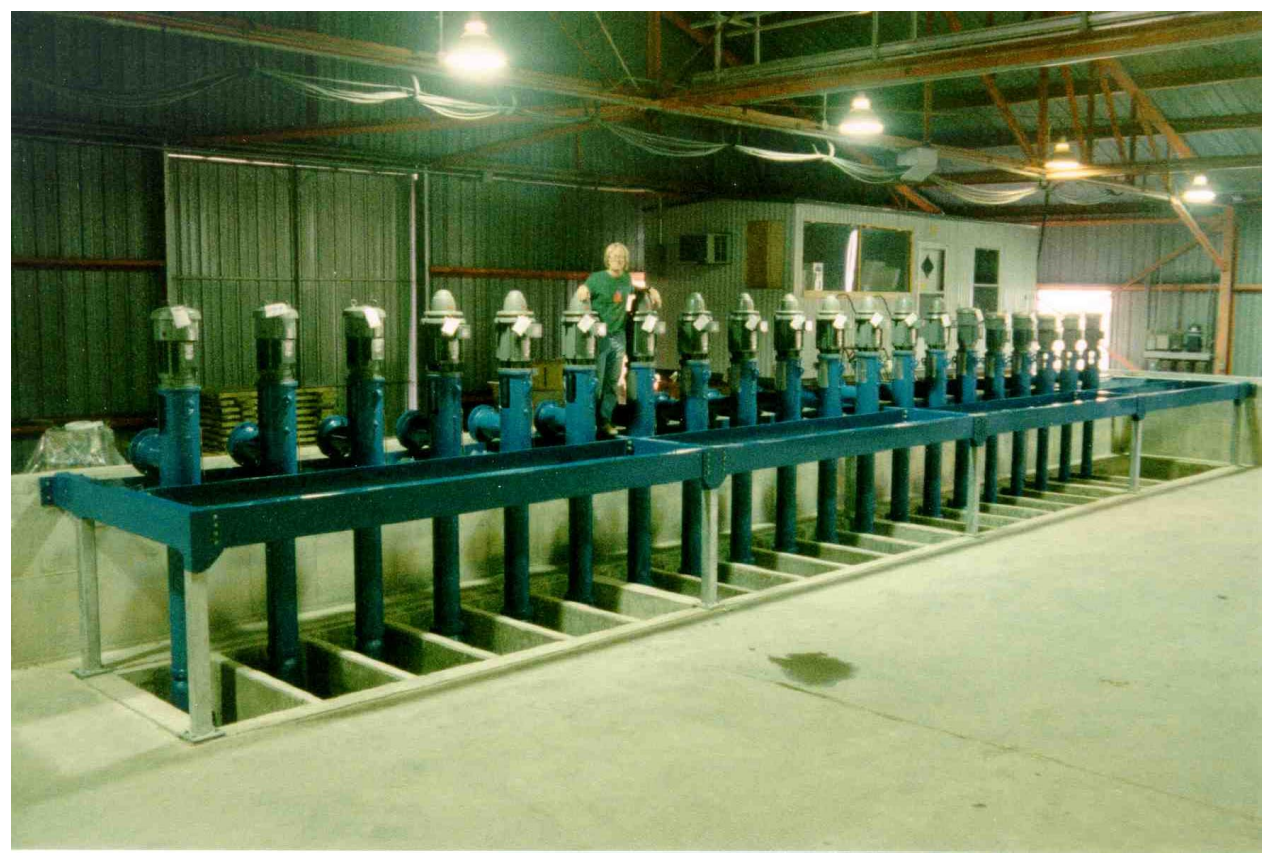

Figure B6. The 20 vertical turbine pumps are mounted inside of the 20 independent sumps to ensure vortex free operation at maximum discharge. Each sump was constructed of reinforced concrete and has dimensions: cross-shore $=0.75 \mathrm{~m}$, longshore $=1.5 \mathrm{~m}$, and depth $=1.2 \mathrm{~m}$

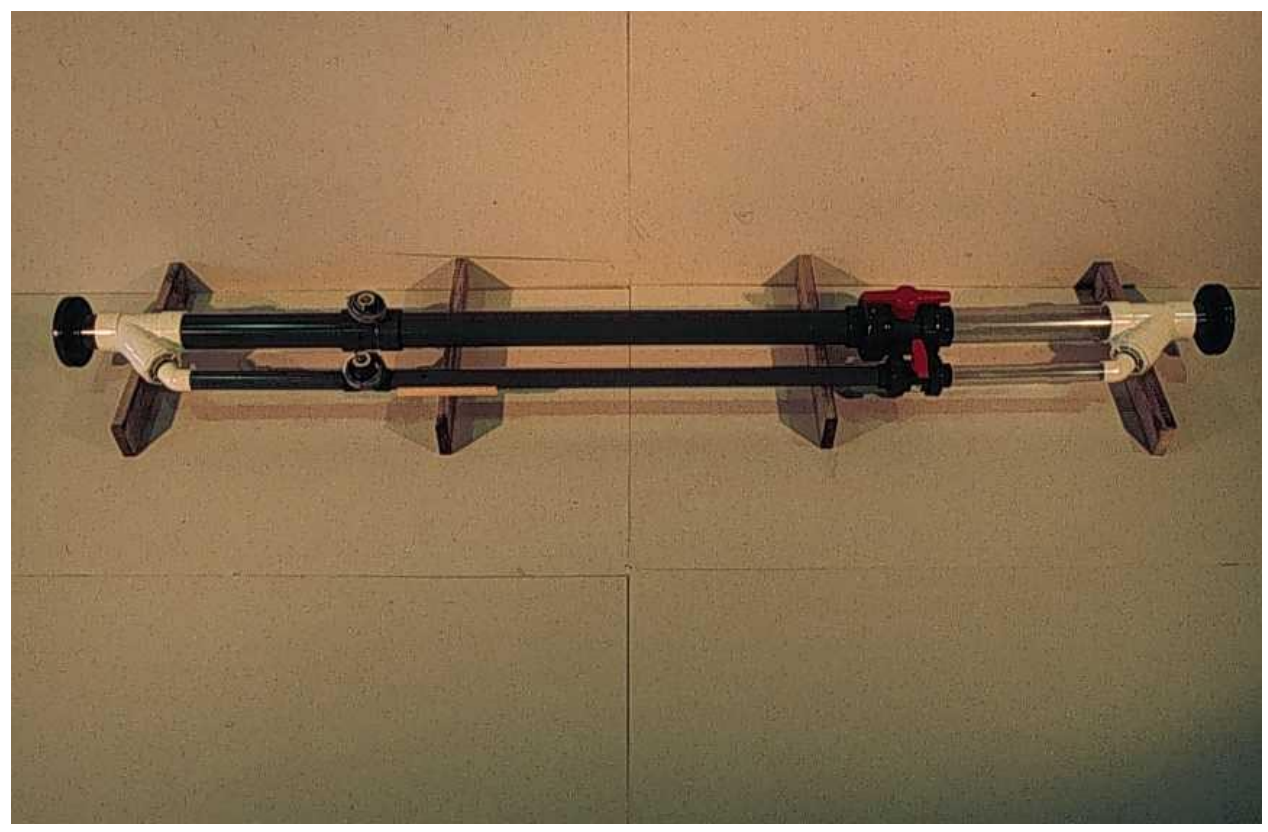

Figure B7. Each pump in the Longshore Current recirculation system pumps water through one of two parallel pipelines. One is for low-flow conditions, the other is for high-flow conditions. The water passes through a valve and then through a flow sensor (from right to left). A 0.3-m-long ruler is shown for scale just upstream of the smaller flow sensor 


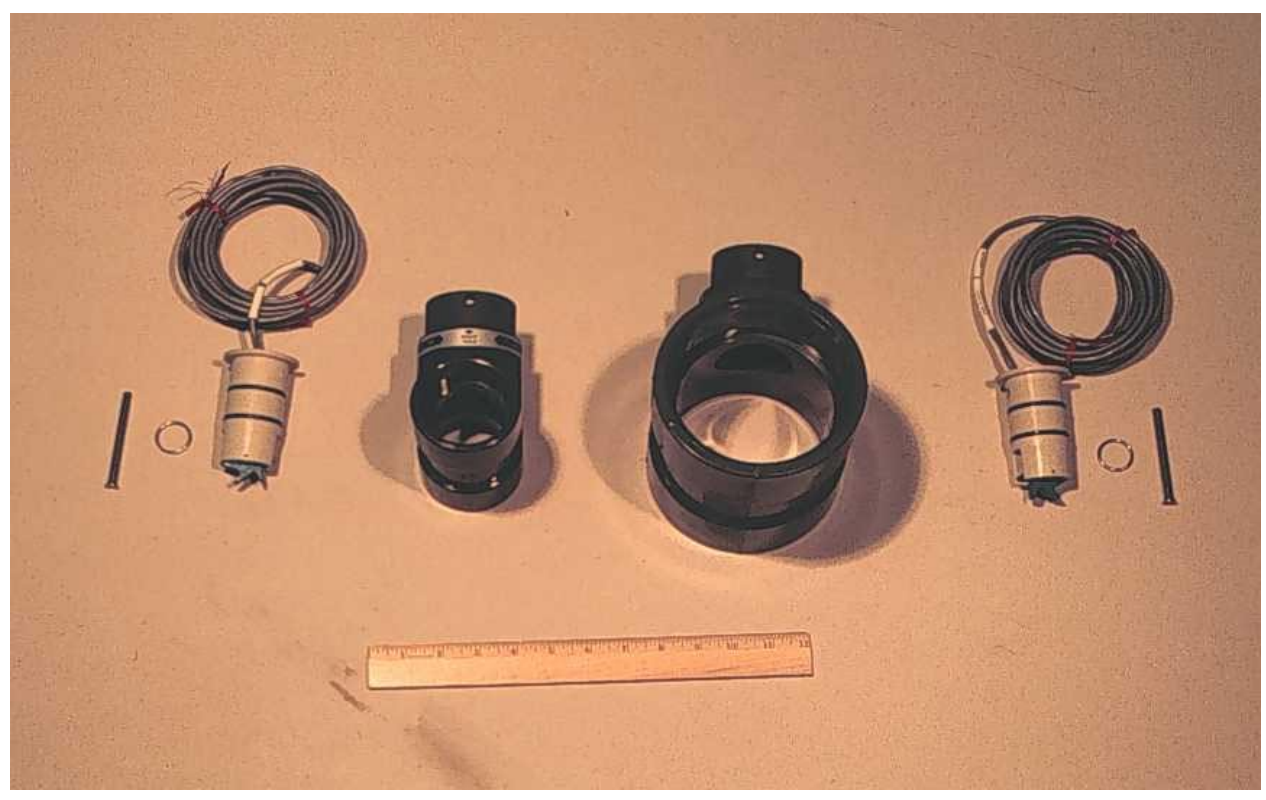

Figure B8. Pump No. 10 forces through either a 50- or a 100-mm-diam in-line flow sensor. The forward-swept design of the impellers, as well as the absence of magnetic drag, provides improved accuracy and repeatability at low flow rates. A 0.3-m-long wooden ruler indicates the physical size of the components

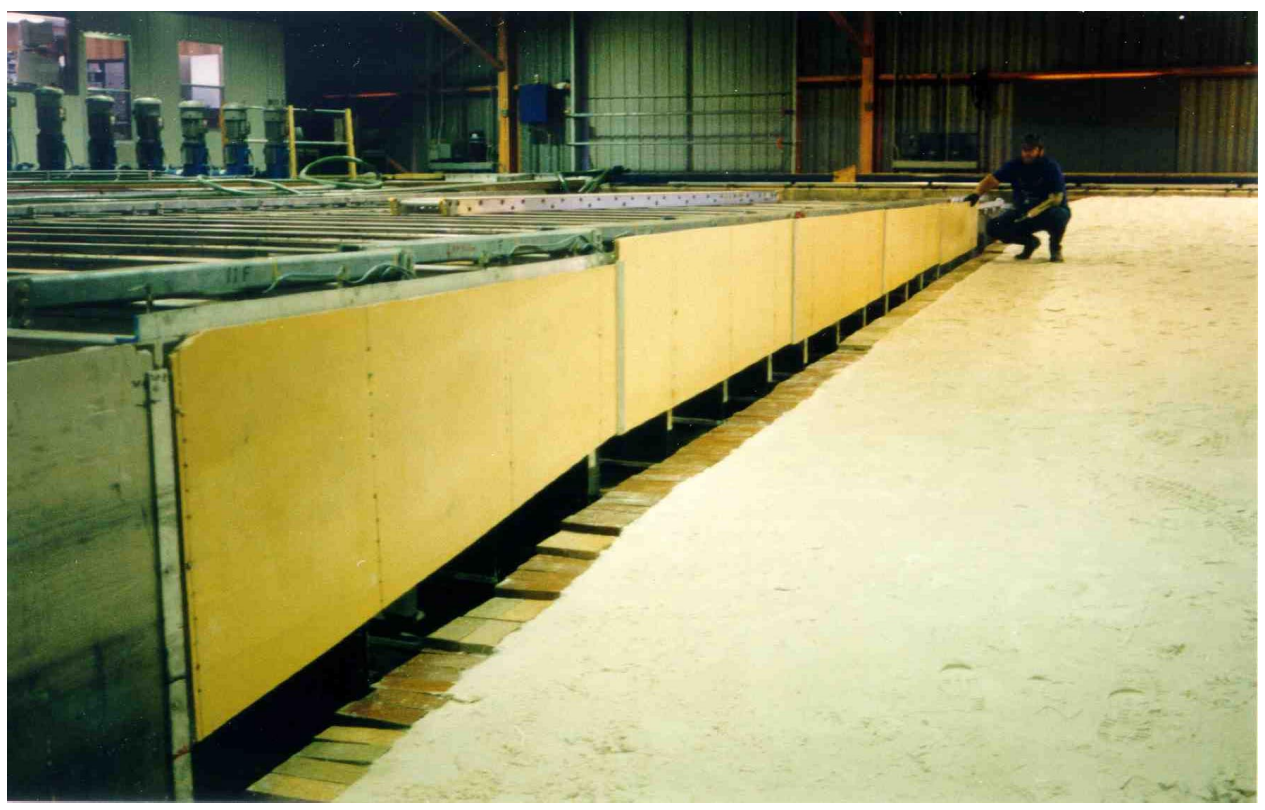

Figure B9. View of the wave guide at the downstream end of the beach. The bottom edge of the wave guide was installed at approximately the minimum wave trough elevation for the maximum wave condition that can be generated in the facility. Hence, the height of the opening beneath the wave guide increases in the offshore direction 


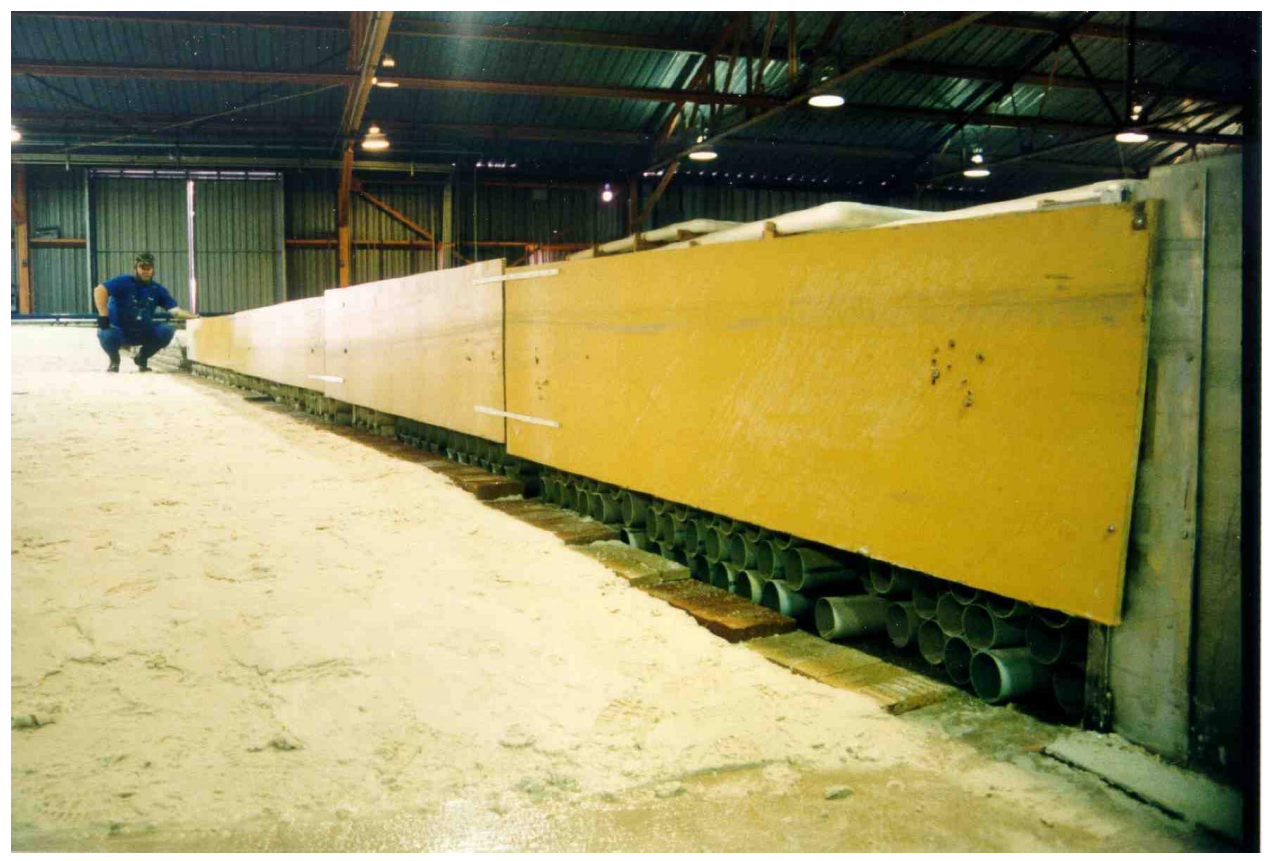

Figure B10. View of the wave guide at upstream end of the beach. The opening beneath the wave guide is $0.15 \mathrm{~m}$ high along the entire length of the wave guide. A matrix of polyvinyl chloride pipe, behind the wave guide, directs the externally recirculated current onto the beach

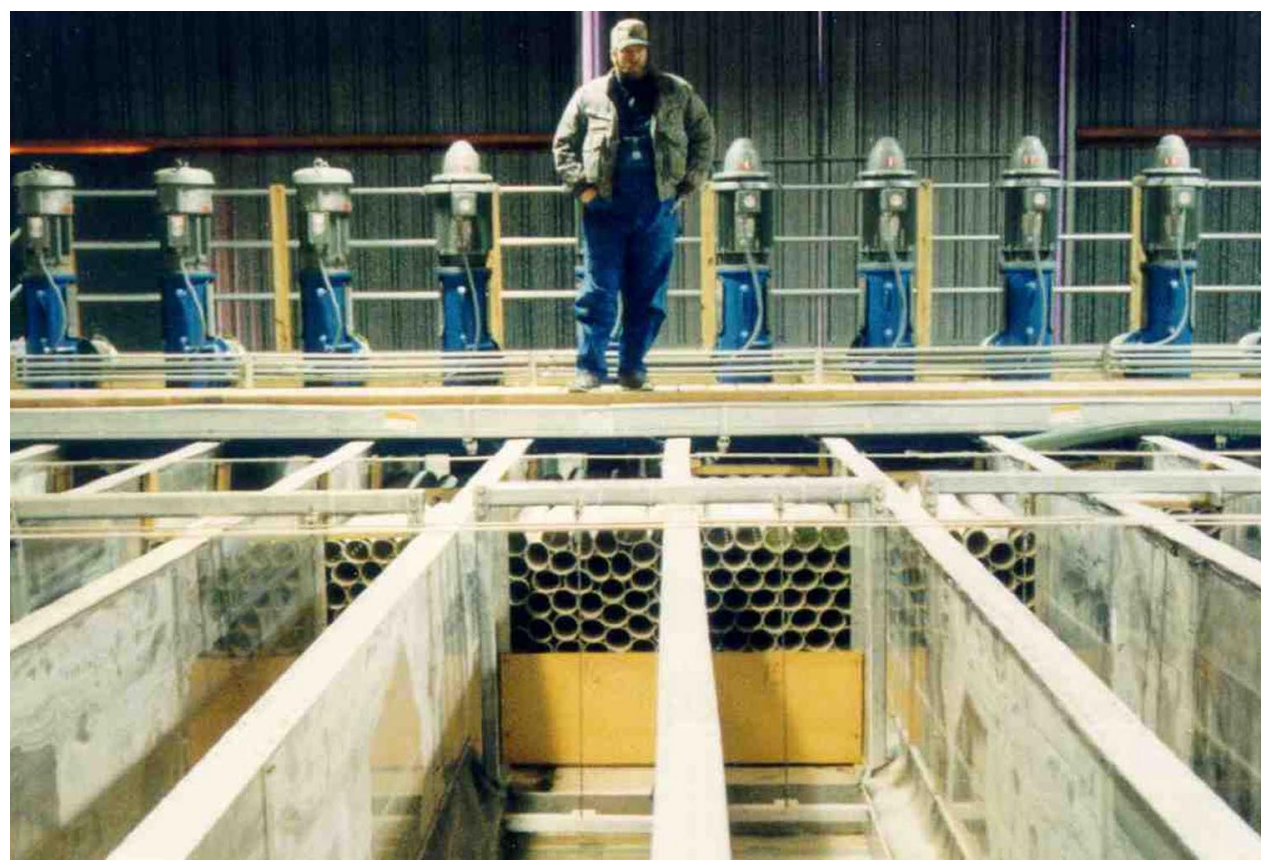

Figure B11. A matrix of PVC pipe was installed immediately upstream of each vertical turbine pump to absorb residual wave energy and to minimize wave reflection in the flow channels. Low-crested weirs were installed in each flow channel to help block any sand that passes over the sediment traps, before it reaches the pumps 


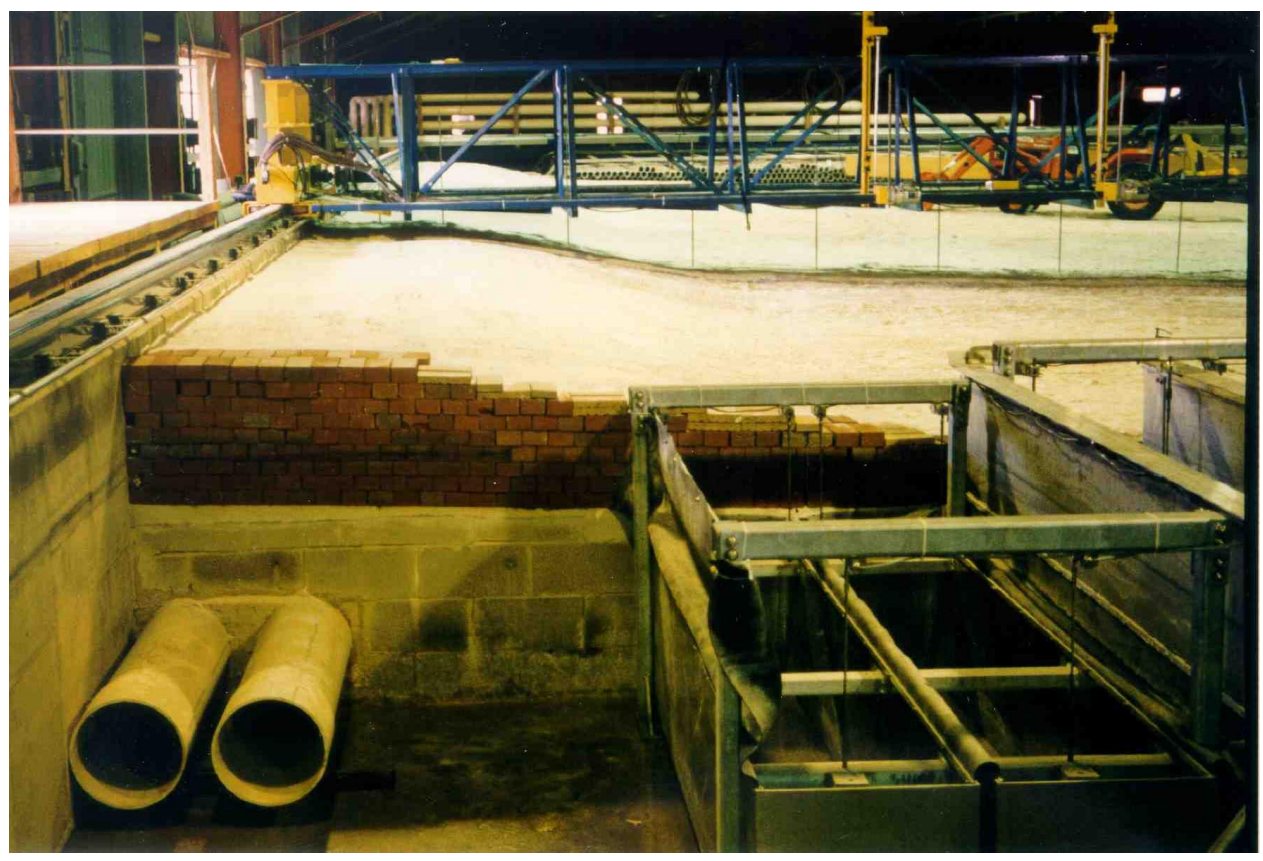

Figure B12. Two 400-mm-diam polyvinyl chloride pipes were installed in the concrete beach, to passively recirculate the longshore current that flows in the swash zone. The two "swash zone traps" (i.e., sand trap No. -1, and -2) can be seen in the bottom right corner of the figure

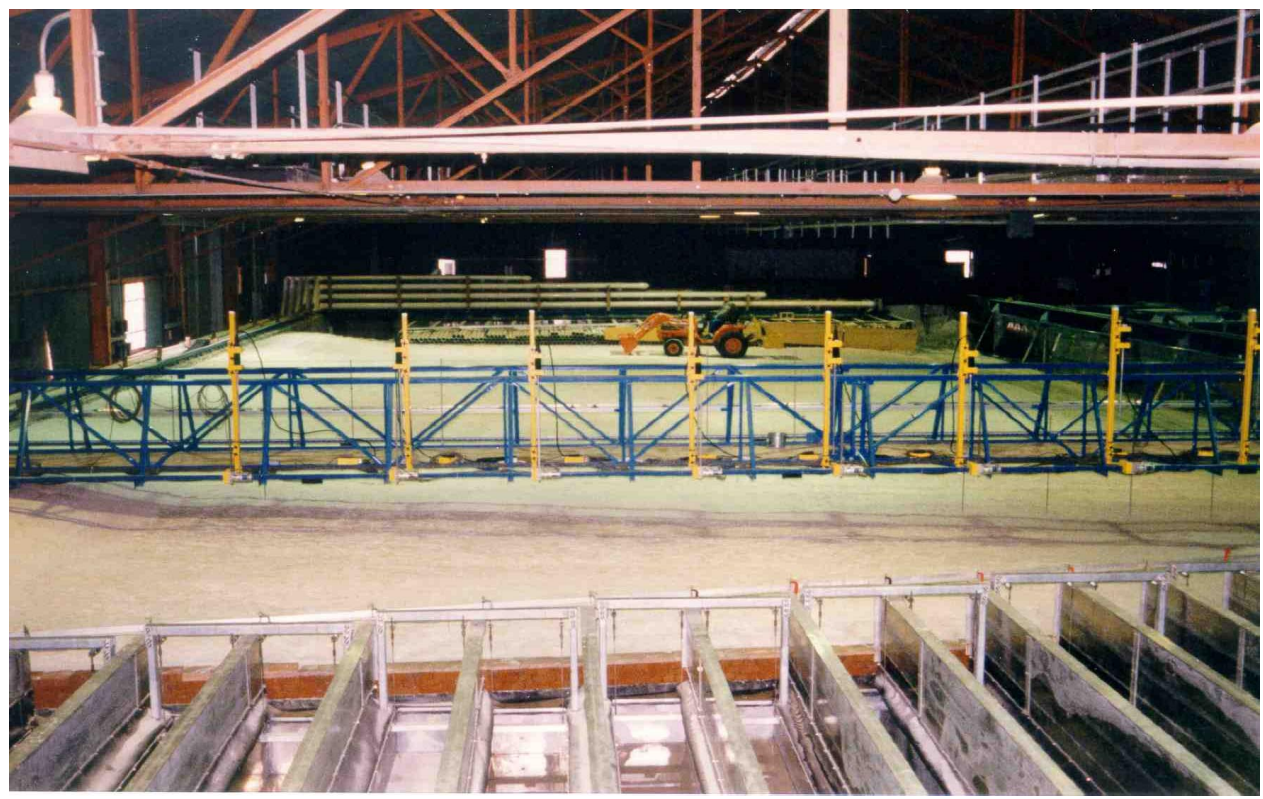

Figure B13. An overall view of the facility after the sand beach has been graded to have straight and parallel depth contours. The sediment traps can been seen near the bottom, with the adjustable boundary along the interface between the sand beach and the sediment traps. Once the tractor is driven out of the facility, the gate is closed and the facility is filled with water to begin an experiment 


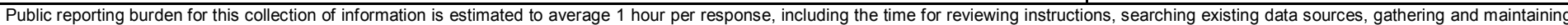

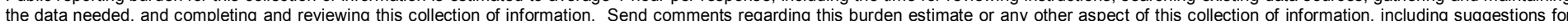

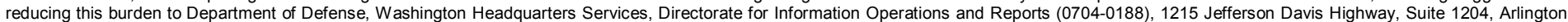

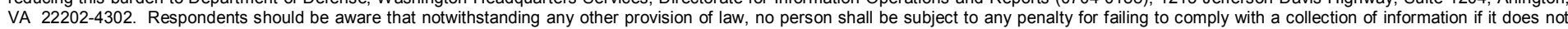
display a currently valid OMB control number. PLEASE DO NOT RETURN YOUR FORM TO THE ABOVE ADDRESS.

\begin{tabular}{|l|l|l}
\hline 1. REPORT DATE (DD-MM-YYYY) & 2. REPORT TYPE & 3. DATES COVERED (FrOm - To)
\end{tabular} \begin{tabular}{l|l} 
September 2001 & Final report
\end{tabular}

4. TITLE AND SUBTITLE

5a. CONTRACT NUMBER

Development of a Large-Scale Laboratory Facility for Sediment Transport

Research

5b. GRANT NUMBER

5c. PROGRAM ELEMENT NUMBER

6. AUTHOR(S)

David G. Hamilton, Bruce A. Ebersole, Ernest R. Smith, Ping Wang

5d. PROJECT NUMBER

5e. TASK NUMBER

5f. WORK UNIT NUMBER

7. PERFORMING ORGANIZATION NAME(S) AND ADDRESS(ES)

8. PERFORMING ORGANIZATION REPORT NUMBER

U.S. Army Engineer Research and Development Center

Coastal and Hydraulics Laboratory

ERDC/CHL TR-01-22

3909 Halls Ferry Road

Vicksburg, MS 39180-6199

\section{SPONSORING / MONITORING AGENCY NAME(S) AND ADDRESS(ES)}

10. SPONSOR/MONITOR'S ACRONYM(S)

Coastal Sedimentation and Dredging Program, General Investigations Research and

Development Program, U.S. Army Corps of Engineers, Washington, DC 20314-1000

11. SPONSOR/MONITOR'S REPORT NUMBER(S)

\section{DISTRIBUTION / AVAILABILITY STATEMENT}

Approved for public release; distribution is unlimited.

\section{SUPPLEMENTARY NOTES}

\section{ABSTRACT}

This report documents the development and initial applications of the Large-scale Sediment Transport Facility (LSTF), which is located at the U.S. Army Engineer Research and Development Center (ERDC), Coastal and Hydraulics Laboratory (CHL), Vicksburg, MS. The LSTF was built to facilitate basic and applied research in the field of coastal sediment transport processes. Work reported here was funded by Work Unit 32870 "Large-Scale Laboratory Investigation of Longshore Sediment Transport," which is under the Coastal Sedimentation and Dredging Program of the General Investigations Research and Development Program of the U.S. Army Corps of Engineers.

A large-scale facility for investigating nearshore and surf zone sediment transport processes has been constructed and successfully applied. This facility provides the Corps of Engineers with a capability for studying longshore sand transport at scales that are much larger than the scales at which previous laboratory research has been conducted to examine these processes. The LSTF is state-of-the-art in terms of the systems that have been built for generating relatively large-scale laboratory waves (four phase-synchronized electricmotor-driven wave generators), pumping and recirculating the longshore current (20 independent and separately controlled pump and piping networks for accurate control of the cross-shore distribution of the current), measuring the quantity of sand that (Continued)

\section{SUBJECT TERMS}

(See reverse.)

\begin{tabular}{|c|c|c|}
\hline \multicolumn{3}{|c|}{ 16. SECURITY CLASSIFICATION OF: } \\
\hline a. REPORT & b. ABSTRACT & c. THIS PAGE \\
\hline UNCLASSIFIED & UNCLASSIFIED & UNCLASSIFIED \\
\hline
\end{tabular}

\begin{tabular}{l|c|c|} 
17. LIMITATION & $\begin{array}{c}\text { 18. NUMBER } \\
\text { OF ABSTRACT }\end{array}$ & 185 \\
& &
\end{tabular}

19a. NAME OF RESPONSIBLE PERSON

19b. TELEPHONE NUMBER (include area code) 


\section{4. (continued)}

moves along the beach (20 instrumented sediment traps), and a fully programmable instrumentation bridge and data acquisition system that includes $10 \mathrm{ADV}$ current sensors, 10 capacitance-type wave gauges, four FOBS arrays, and an automated beach profiling system. All facility components were designed to maximize accuracy of data acquired in movable-bed experiments and minimize the amount of human labor (and time) required to perform them.

The LSTF has proven to be an excellent and robust facility and, so far, has yielded unprecedented measurements of surf zone sediment transport processes in a laboratory setting, including sand transported in suspension. The potential for making R\&D advancements through the use of the facility is quite high.

\section{5. (continued)}

Laboratory study

Large-scale laboratory facility

Longshore currents

Longshore sediment transport

Movable-bed model

Nearshore sediment transport

Physical modeling

Sediment transport processes

Surf zone currents

Surf zone processes

Wave and current measurements

Wave breaking 